volume 8

ISSUE 1

2020 gennaio-febbraio

Editor in Chief Michele Meschi

Supervisor Editor Roberto Nardi

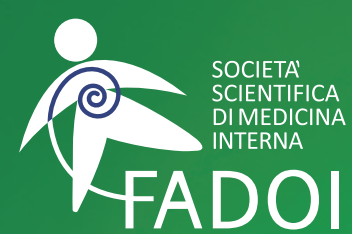

FEDERAZIONE

DELLE ASSOCIAZIONI

DEI DIRIGENTI

OSPEDALIERI

INTERNISTI

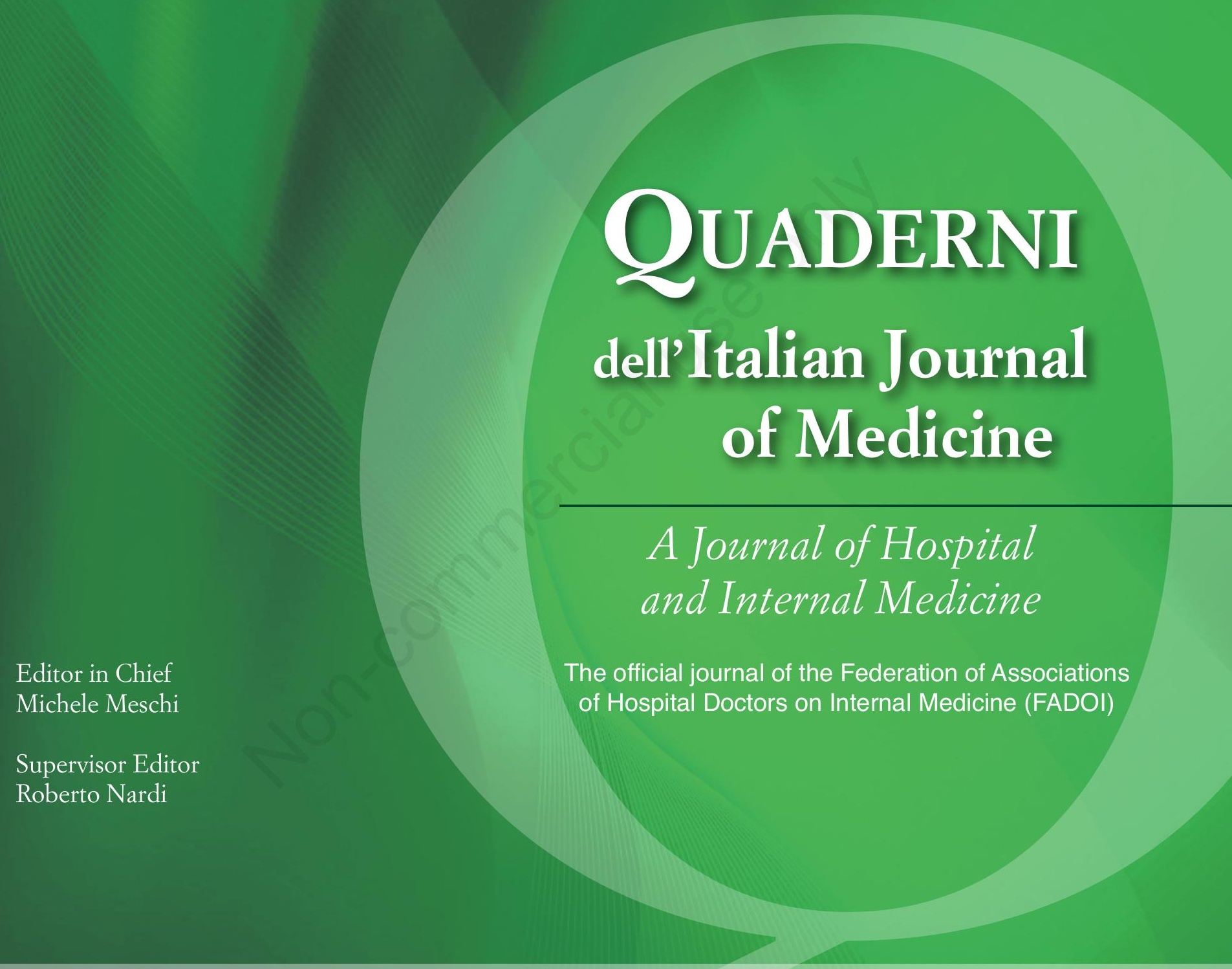

\title{
Metodologia clinica oggi nel paziente internistico
} complesso

Guest Editors: Roberto Nardi, Paola Gnerre, Andrea Fontanella, Dario Manfellotto 
PRESIDENTE ELETTO

Francesco Dentali, Varese, Italy

\section{PAST PRESIDENT}

Andrea Fontanella, Napoli, Italy

\section{SEGRETARIO}

Paola Gnerre, Savona, Italy

\section{STAFF DI SEGRETERIA}

Maurizia Gambacorta, Todi (PG), Italy

Ada Maffettone, Napoli, Italy

Claudia Tieri, Bari, Italy

\section{TESORIERE}

Giorgio Ballardini, Rimini, Italy

\section{COORDINATORE COMMISSIONE GIOVANI}

Ombretta Para, Firenze, Italy

\section{RESPONSABILE RAPPORTI ISTITUZIONALI}

Claudio Santini, Roma, Italy

\section{RESPONSABILE RAPPORTI CON LE REGIONI}

Alberto Fortini, Firenze, Italy

\section{RESPONSABILE EVENTI E INIZIATIVE SPECIALI}

Mauro Campanini, Novara, Italy

EDITOR IN CHIEF ITALIAN JOURNAL OF MEDICINE

Giorgio Vescovo, Padova, Italy
RESPONSABILE DEI

QUADERNI DELL'ITALIAN JOURNAL OF MEDICINE EDIZIONI ON LINE

Michele Meschi, Borgo Val di Taro (PR), Italy

SUPERVISOR EDITOR DEI

QUADERNI DELL'ITALIAN JOURNAL OF MEDICINE

Roberto Nardi, Bologna, Italy

RESPONSABILE SITO WEB E COMUNICAZIONE SOCIAL

Salvatore Lenti, Arezzo, Italy

Maurizia Gambacorta, Todi (PG), Italy

WEB MANAGER E CONTENT EDITOR

Giuseppe Oteri, Milano, Italy

Davide Ghilardi, Milano, Italy

RESPONSABILE SISTEMA GESTIONE QUALITÀ

Franco Berti, Roma, Italy

DPO

Alba Sciascera, Magenta (MI), Italy

\section{CONSULTA DEI PRESIDENTI}

Sandro Fontana, Biella, Italy

Salvatore Di Rosa, Palermo, Italy

Ido Iori, Reggio Emilia, Italy

Giovanni Mathieu, Pinerolo (TO), Italy

Antonino Mazzone, Legnano (MI), Italy

Carlo Nozzoli, Firenze, Italy

Mauro Campanini, Novara, Italy

Andrea Fontanella, Napoli, Italy 


\section{Italian Journal of Medicine}

\section{COORDINATORE}

David Terracina, Roma, Italy

\section{SEGRETARIO}

Andrea Montagnani, Grosseto, Italy

\section{DIRETTORI DEL DIPARTIMENTO DELLA RICERCA CLINICA FADOI}

Filippo Pieralli, Firenze, Italy

Fulvio Pomero, Savigliano (CN), Italy

DIRETTORI DIPARTIMENTO

\section{PER LA FORMAZIONE E AGGIORNAMENTO}

Luigi Magnani, Voghera (PV), Italy

Roberta Re, Novara, Italy

\section{COORDINATORE SCIENTIFICO CENTRO STUDI FONDAZIONE FADOI}

Gualberto Gussoni, Milano, Italy

\section{DELEGATI SOCIETÀ SCIENTIFICHE COLLEGATE}

FISM Antonino Mazzone, Legnano (MI), Italy

SIF Luigi Magnani, Voghera (PV), Italy

Consulta Mauro Campanini, Novara (MI), Italy

cardiovascolare Michele Stornello, Siracusa, Italy

SIIA e ISO Michele Stornello, Siracusa, Italy Arcangelo Iannuzzi, Pomigliano d'Arco (NA), Italy

EFIM

Antonio Brucato, Milano, Italy

Lorenza Lenzi, Pomarolo (TN), Italy

Choosing wisely Roberto Frediani, Chieri (TO), Italy

\section{PROGETTI SPECIALI}

AGGIORN@FADOI

Giuliano Pinna, Marco Grandi

PROGETTO NUOVE TECNOLOGIE

Francesco Nasso, Flavio Tangianu

PROGETTO MEDICINA DI GENERE

Cecilia Politi

PROGETTO COMPETENCE

Flavio Tangianu

PROGETTO GOVERNANCE

Stefano De Carli, Andrea Montagnani, Fabrizio Colombo

PROGETTO HOSPITALIST

Francesco Orlandini

PROGETTO FINE VITA

Mauro Carbone, Fabio Gilioli

PROGETTO GASTROENTEROLOGIA \& FEGATO

Luca Fontanella, Paola Piccolo, Franco Radaelli, Giancarlo Parisi

PROGETTO NUTRIZIONE CLINICA

Roberto Risicato, Luciano Tramontano

PROGETTO MALATTIE INFETTIVE/ANTIBIOTICI

Claudio Santini, Massimo Giusti, Marco Falcone

PROGETTO ECOGRAFIA INTERNISTICA

Francesco Cipollini, Nicola Mumoli

PROGETTO MALATTIE RARE

Antonio Brucato, Antonella Paradiso

PROGETTO BPCO/NIV

Marco Candela, Giuseppe De Matthaeis, Francesco Ventrella

PROGETTO TROMBOSI

Mauro Silingardi, Matteo Giorgi Pierfranceschi, Pierpaolo Di Micco

PROGETTO SDO

Giovanni Mathieu

PROGETTO TRIAL

Giancarlo Agnelli, Antonio Ceriello, Leo Fabbri, Claudio Ferri,

Franco Radaelli, Paolo Verdecchia

\section{PRESIDENTE ANÍMO}

Gabriella Bordin, Castelfranco Veneto (TV), Italy 


\title{
QUADERNI - Italian Journal of Medicine
}

\author{
METODOLOGIA CLINICA OGGI NEL PAZIENTE INTERNISTICO \\ COMPLESSO \\ Guest Editors: R. Nardi, P. Gnerre, A. Fontanella, D. Manfellotto
}

INTRODUZIONE

Introduzione alla lettura

RASSEGNE

A. Fontanella, P. Gnerre

Il metodo clinico classico della tradizione italiana

I. Portioli

Modelli di ragionamento clinico e strumenti utilizzati nella pratica clinica in ambito internistico ai fini decisionali . . . . . .

P. Gnerre, R. Nardi

I difetti del ragionamento clinico come causa di errori diagnostici . . . 31 M. La Regina, F. Orlandini

Metodologia clinica e EBM: vantaggi e limiti nel mondo reale dei pazienti complessi - II mondo reale.

T.M. Attardo

Metodologia clinica e EBM: vantaggi e limiti nel mondo reale dei pazienti complessi - Trappole e possibili bias . . . . . . . . . . . .

M. Cavalleri

Significatività statistica e rilevanza clinica.

D. Tirotta

Dalla medicina personalizzata alla medicina di precisione

G. Gensini, N. Scarpa

Una possibile alternativa alle decisioni:

il metodo Clinical Multi-criteria Decision Assessment (CMDA) . . . . .

O. Para

Il metodo clinico in condizioni di incertezza:

errori, vincoli, esperienza, buon senso e le opinioni del paziente. . . . M.L. Cipollini, E. Romboli

Meta-cognizione e capacità di decision making

F. Gilioli

Complessità, caos e ragionamento clinico

F. Tangianu, S. Corrao, A. Mazzone, P. Gnerre, G. Scanelli, M. Gambacorta,

D. Tirotta, A. Fontanella, M. Adamo, R. Nardi

Competenza e metodologia in Medicina Interna:

quali strumenti di valutazione delle capacità di ragionamento clinico?

Prima Parte . . . . . . . . . . . . . . . . . . . . .

F. Tangianu, A. Mazzone, G. Pinna, G. Chesi, P. Gnerre, F. Berti, M. Stornello, C. Nozzoli, S. Corrao, M. Silingardi, G. Mathieu, A. Fontanella, D. Borioni, R. Nardi 


\section{QUADERNI - Italian Journal of Medicine}

Competenza e metodologia in Medicina Interna:

quali strumenti di valutazione delle capacità di ragionamento clinico?

Seconda Parte

F. Tangianu, A. Mazzone, G. Pinna, G. Chesi, P. Gnerre, F. Berti, M. Stornello,

C. Nozzoli, S. Corrao, M. Silingardi, G. Mathieu, A. Fontanella, D. Borioni,

R. Nardi

La semeiotica medica: presente e futuro 


\title{
Introduzione alla lettura
}

\author{
Andrea Fontanella ${ }^{1}$, Paola Gnerre ${ }^{2}$ \\ ${ }^{1}$ Ospedale Fatebenefratelli, Napoli; ${ }^{2}$ Ospedale San Paolo, Savona, Italia
}

«Il metodo clinico ha come primo obiettivo il conoscere o, meglio, il riconoscere come premessa al prevedere e al decidere» (Torsoli 1997). ${ }^{1} \mathrm{La}$ metodologia clinica è lo studio degli orientamenti di pensiero e delle regole che presiedono all'esercizio razionale della Medicina. La finalità del suo insegnamento è l'acquisizione di un corretto modo di procedere da parte del professionista della salute, che sarà tale solo se in grado di coniugare la conoscenza alla capacità di agire. ${ }^{2}$ Questo numero monografico, partendo dalla definizione del metodo clinico classico, ripropone i vari modelli di ragionamento clinico guidando il lettore tra le possibili aree di incertezza, tra i vantaggi e i limiti nell'applicabilità nel mondo reale dei pazienti complessi, il ruolo dell'evidence based management (EBM) e delle nuove tecnologie nel processo diagnostico fino ad affrontare l'annoso problema della valutazione delle competenze metodologiche. Proprio nel campo dell'EBM è sempre più necessario fare una distinzione tra EBM Epistemologica e quella Pratica. ${ }^{3}$ La prima enfatizza la conoscenza probabilistica acquisita attraverso i trial clinici (RCT), preferendola ad altre forme di conoscenza (dati di metanalisi invece che intuizione, autorità del clinico, fisiopatologia). La seconda si occupa di trovare modalità efficaci ed efficienti per incorporare la migliore conoscenza disponibile nella pratica clinica corrente (linee guida, audit clinico, outcome research). ${ }^{4}$

È questa la vera motivazione che ci ha spinti a realizzare una monografia proprio sulla metodologia clinica, nata dall'idea e dalla volontà del Dr Nardi e per rispondere a questa esigenza è necessario fare alcune premesse.

Corrispondente: Paola Gnerre, Ospedale San Paolo Savona, Italia.

E-mail: pgnerre@yahoo.it

Articolo pubblicato secondo la Creative Commons Attribution NonCommercial 4.0 License (CC BY-NC 4.0).

${ }^{\circ}$ Copyright: the Author(s), 2020

Licensee PAGEPress, Italy

QUADERNI - Italian Journal of Medicine 2020; 8(1):1-2
Secondo la definizione dell'Unione Europea dei Medici Specialisti (UEMS) l'atto medico comprende tutte le attività professionali, ad esempio di carattere scientifico, d'insegnamento, di formazione, educativa, organizzativa, clinica e di tecnologia medica, svolte al fine di promuovere la salute, prevenire le malattie, effettuare diagnosi e prescrivere cure terapeutiche o riabilitative nei confronti di pazienti, individui, gruppi o comunità, nel quadro delle norme etiche $e$ deontologiche. ${ }^{5}$ L'atto medico è, pertanto, legato a obiettivi ben definiti che includono la descrizione e l'identificazione del quadro morboso, l'approccio eziopatogenetico per interpretare segni e sintomi, la formulazione diagnostica della malattia o della sindrome, tutte cose che conducono alla scelta dell'intervento terapeutico in grado di definire la prognosi e l'evoluzione del decorso clinico. La formulazione diagnostica è, pertanto, il momento critico conclusivo di una procedura osservativo-logica che consente la definizione di una malattia.

La metodologia clinica è un elemento fondamentale della medicina. È il processo con cui, attraverso la valutazione della storia del paziente, dell'esame obbiettivo e della revisione dei dati disponibili, si identificano la natura e la causa di una malattia. Questa definizione descrive due fasi distinte, la prima, dell'acquisizione da elementi percepibili - il colloquio, il contatto, $i$ dati materiali chimici e strumentali -, una seconda fase, immateriale, in cui si svolgono i processi mentali coinvolti nel momento decisionale e quindi il ragionamento clinico (Portioli). ${ }^{6}$ Il moltiplicarsi di indagini strumentali e laboratoristiche ha notevolmente facilitato il compito del medico nell' individuazione della diagnosi. Questo però ha sempre più incrementato la convinzione secondo cui la diagnosi scaturisce frequentemente dalle indagini di laboratorio o da quelle strumentali portando alla dispendiosa, dispersiva e talvolta esasperata ricerca del dato di laboratorio/strumentale rivelatore della diagnosi. La rigorosa e pedissequa applicazione dell'EBM implica un approccio meccanicistico basato sugli algoritmi, simile ai programmi informatici di intelligenza pre-artificiale primitiva del passato. In tale approccio, il medico vede il paziente come una statistica piuttosto che un 
individuo. Questo tipo di medicina potrebbe essere praticata dagli amministrativi e, ertamente, non è applicabile nell'attuale mondo fatto da pazienti cronici e complessi. Nel mondo reale, tuttavia, gli studi clinici possono dire quali trattamenti sono efficaci per quella patologia, ma non necessariamente quali pazienti dovrebbero riceverli (Hampton JR). ${ }^{7}$

A differenza della semeiotica, la metodologia clinica prescinde totalmente dai contenuti della medicina, stabilendo le regole del procedimento clinico. La metodologia clinica, quindi, rappresenta un settore fondamentale delle conoscenze mediche insegnando a fare il miglior uso dei rilievi clinici forniti dalla semeiotica.

Ma mentre la medicina si è dedicata con sempre maggior attenzione alla prima fase, quella della semeiotica medica, poco o nullo è stato l'interesse per lo studio, la ricerca e l'insegnamento delle regole metodologiche. Si comprende così come anche nell'ordinamento degli studi universitari la semeiotica medica abbia potuto assurgere a dignità d'insegnamento autonomo, mentre fino alla recente riforma degli studi di medicina, l'insegnamento della metodologia clinica sia stato sempre associato, senza costituirne parte risaltante, alla patologia speciale medica, cioè alla descrizione dei modelli di malattia. ${ }^{1}$

Per concludere, in una società in cui tutto deve essere quantificabile e misurabile è possibile valutare la competenza nell'ambito del ragionamento clinico? (Tangianu et al.). ${ }^{8}$ Anche perché, come dice Giorgio Cosmacini: La medicina non è una scienza, è una pratica basata su scienze che opera in un mondo di valori. ${ }^{9}$

La valutazione delle capacità di ragionamento clinico costituisce un problema complesso. Un singolo strumento non è mai sufficiente - di per sé - per valutare il ragionamento diagnostico. Viceversa, un programma di valutazione che impieghi più strategie integrate può fornire un processo più efficace per determinare la competenza del medico nel ragionamento diagnostico. Questo è un ulteriore tema per la ricerca futura, su cui le Società Scientifiche potranno impegnarsi. ${ }^{8}$

Ecco perché è necessaria un'attenta lettura di questo numero monografico... ne abbiamo tutti da imparare.

\section{Bibliografia}

1. Torsoli A. Manuale di metodologia clinica. Roma: Il Pensiero Scientifico; 1997.

2. Ferraris M. Pensiero in movimento. Torino: Paravia Editore; 2019.

3. Serio A, Cicala M. La metodologia clinica nella formazione del medico. MEDIC 2013;21:75-81.

4. Sackett DL, Rosenberg WM, Gray JA, et al. Evidence based medicine: what it is and what it isn't. BMJ 1996;312:71-2.

5. FNOMCeO. UEMS: la Definizione europea dell'atto medico; 2005-2006. Disponibile su: https://portale. fnomceo.it/uems-la-definizione-europea-dellatto medico/

6. Portioli I. Il metodo clinico classico della tradizione italiana. Quad Ital J Med 2020; 8(1):3-18.

7. Hampton J. Therapeutic fashion and publication bias: the case of anti-arrhythmic drugs in heart attack. J R Soc Med 2015;108:418-20.

8. Tangianu F, Mazzone A, Pinna G, et al. Competenza e metodologia in Medicina Interna: quali strumenti di valutazione delle capacità di ragionamento clinico? Prima parte e Seconda parte. Quad Ital J Med 2020; 8(1):91-124.

9. Cosmacini G. La medicina non è una scienza. Breve storia delle sue scienze di base. Milano: Raffaello Corina Editore; 2008. 


\title{
Il metodo clinico classico della tradizione italiana
}

\author{
Italo Portioli
}

Commissione Farmaci Area Vasta Emilia Nord (AVEN), IRCCS Arcispedale S. Maria Nuova, Reggio Emilia

\section{Premessa}

Il metodo clinico è il processo con cui, attraverso la valutazione della storia del paziente, dell'esame obbiettivo e della revisione dei dati disponibili, si identificano la natura e la causa di una malattia. Questa definizione descrive due fasi distinte, la prima, della acquisizione da elementi percepibili - il colloquio, il contatto, i dati materiali chimici e strumentali - , una seconda fase, immateriale, in cui si svolgono i processi mentali coinvolti nel momento decisionale. I passaggi iniziali della prima, l'anamnesi e l'esame obbiettivo, hanno avuto nel modello classico un fasto vicino alla esclusività, e ora questo fastigio è opaco (scuro, per Dante). La seconda, del momento decisionale, seguiva invece percorsi mentali obbligati, allora meno gravati di conoscenze in cui districarsi. La differente natura delle due fasi - mutevole nel primo passaggio, obbligata nel secondo - ha condizionato, in questa revisione, una loro trattazione asimmetrica. Essa sarà in gran parte dedicata al ruolo fondamentale che nel metodo classico hanno avuto la storia clinica e la visita del paziente. Il loro peso fu tale da generare un rapporto medicopaziente olistico, inteso come rispetto non solo della unità organica del corpo ma della totalità armoniosa della persona, in soma e psiche. La parte finale ne propone una attualizzazione argomentata, nella prospettiva di un suo ripristino nella pratica medica.

\section{Il metodo clinico: acquisizione in movimento (non patrimonio consegnato)}

Definire il metodo clinico della tradizione classica italiana significa stabilirne precisi caratteri, peculiarità

Corrispondente: Italo Portioli, Commissione Farmaci Area Vasta Emilia Nord (AVEN), IRCCS Arcispedale S. Maria Nuova, Reggio Emilia, Italia.

E-mail: Italo.Portioli@ausl.re.it

Articolo pubblicato secondo la Creative Commons Attribution NonCommercial 4.0 License (CC BY-NC 4.0).

${ }^{\circ}$ Copyright: the Author(s), 2020

Licensee PAGEPress, Italy

QUADERNI - Italian Journal of Medicine 2020; 8(1):3-18 rispetto alle scuole di Medicina europee - e non solo e ai loro assunti, precisarne confini, in una parola disegnarne una riconoscibilità non equivoca. Ma come costruire una simile specificità? Da dove prendere le mosse per questa ricerca? Le risposte possibili sono diverse ma da un incipit così serioso ci si attenderebbe una linea di piena ortodossia, che si attenga cioè a dottrina consolidata e storicizzata. È possibile tuttavia anche una seconda risposta, più pragmatica, quella di ripercorrere, per rispondere, la esperienza personale e provare a carpirne il come, nel periodo del metodo classico, si è diventati medici, cercare lì le tracce di un principio unificante che possa essere definito: metodo.

La prima soluzione è per me controintuitiva. Confesso, non ho attrazione per i modelli metodologici. Non li ho mai sentiti come regole cui rispondere o come guide da seguire. Il fatto è che nessuno mi ha mai insegnato - intendo nel senso di materia didattica organizzata - che prima di mettere mani a un malato era necessario avere chiarezza sui ferri del mestiere e sul modo più cauto di maneggiarli. Oggi è diverso. Agevolmente dissertiamo sui fondamenti estrattivi della esperienza (induzione o deduzione), sulle insidie degli errori sistematici (i bias, in qualunque sito del procedimento si annidino), sulle attitudini psicologiche non derogabili con cui avvicinare il paziente (l'empatia, la umanizzazione), sulle strategie del come procedere ragionatamente per arrivare alla conclusione diagnostica (per flow charts, per algoritmi, guidati dall'evento di partenza). Si tratta di armi con cui in questi anni si è costruito un poderoso armamentario logico. Ma quando e dove l'abbiamo studiata e appresa una dottrina assimilabile a questa? Un pregiudizio personale, ingenuo forse ma confermato, anche amaramente, nel tempo è che si conoscono e si apprendono, nel senso che si vive in confidenza e intimità mentale, solo le materie per cui si è dovuto dare un esame. Ora, abbiamo avuto per mano, da studenti o da giovani incerti medici, un testo amico che ci introducesse a questi segreti di metodo su cui studiare e formarci? Siamo stati mai interrogati, nel senso basico e letterale di abbiamo mai dato un esame formale, su temi di metodologia clinica? $\mathrm{O}$, in mancanza di un libro, abbiamo almeno incontrato, nei nostri primi contatti da studenti con le sale (allora: stanzoni) di degenza, un Assistente che ne avesse ben 
in mente i presupposti e fosse sufficientemente illuminato e generoso da mettercene a parte? La risposta è, almeno per me, negativa. Non era così. Non si insegnava nessun metodo. Non esistevano solfeggi, disamoranti, prima del sospirato accesso a fare musica viva, non indicazioni teoriche di percorsi. Non si dava guida alcuna.

Altro era infatti lo spirito. Superati i faticosi anni propedeutici, ci si muoveva con il desiderio di imparare il più presto possibile sul malato. E qui si affaccia la seconda possibilità di metodo, del provare a ripercorrere in modo ordinato la propria diretta esperienza, chiamiamola formativa. La parola d'ordine, implicita, era partire dalla clinica. Così, siamo stati gettati, ci siamo gettati nella clinica, nei reparti, cercando un alleato che ci insegnasse, senza accademia, come ci si accosta a un paziente e come lo si visita. Il learning by doing che poi sarebbe diventato un refrain, era il nostro fondamento di lavoro. Disordinato? O il popolare precetto anglosassone era solo una ripresa tardiva di Vico, che nella ricognizione concettuale più recente operata da Ferraris $^{1}$ della Scienza nuova, aveva sostenuto che il fare precede il sapere (Homo non intelligendo fit omnia) $\mathrm{e}$ precedendolo lo permette? Addirittura, non pensavamo proprio che, al di fuori della clinica, e del suo palpito, potesse esistere una sapienza di fondo, che la ripensasse quasi in astratto. Solo con il tempo, appunto, abbiamo costruito e continuamente ricostruito i passaggi del nostro approccio al paziente. Il metodo clinico era dunque - prima considerazione personale - un processo in movimento, che si alimenta soprattutto in forza di due stimoli non paralleli, il grado di maturazione professionale conquistata e le circostanze. Seguendo, non sempre per scelta, le diverse occasioni di lavoro - in ambiente accademico, in un ospedale grande o piccolo, in un pronto-soccorso $\mathrm{o}$ in medicina dì urgenza, in un setting di clinica molto o meno intensivo o di lungo-degenza - abbiamo visto via via rinnovarsi $i$ nostri modelli, attuarsi un programma senza programma. Questo, per dire che i modelli si muovono con noi, crescono e cambiano con noi. Di volta in volta impieghiamo - in modo intuitivo? - quello, tra gli approcci che poi saranno dogmatizzati, che abbiamo sperimentato essere il più congruente con la situazione clinica che ci si presenta e con il livello di maturità professionale raggiunto.

Viene in mente, per lontana analogia, - spero non supponente o blasfema - il modo in cui si formano le teorie filosofiche e il corpo stesso delle dottrine religiose; queste non sono calate nel mondo fatte e compiute da una divinità che le elargisce dall'alto, ${ }^{2}$ come un sistema compatto di postulati e di asserzioni, ma appunto come un corpo che prende di continuo nuova anima dalla spinta delle risorse di pensiero che il tempo trasmette.
Si può dire - se il discorso regge - che le teorizzazioni che, con grande forza speculativa e di argomentazione, sono state proposte nel passato ma anche, nel nostro recente presente ${ }^{3,4}$ per interpretare $\mathrm{i}$ comportamenti clinici e portarli alla dignità di modelli, sono comunque, sempre, operazioni a posteriori, elaborazioni a freddo (siamo già alla mia seconda inosservanza). Detto in altra versione, l'ordinare in termini di dottrina di pensiero i processi che portano alla diagnosi è solo il segno di quanto siano aperti, agevoli e molteplici gli spazi mentali in cui ci avventuriamo prima di poter pensare di aver fatto un passo nella direzione della diagnosi giusta. Le personalità in grado di organizzare, sia pure a posteriori, questi approcci come dottrina ripetibile e universalmente applicabile, sono figure dotate di menti speciali, di robusta cultura filosofica e portate al ragionare classificativo-categoriale. Nello sforzo di ordinare lo svolgersi volatile degli eventi diagnostici, nel carpirne $\mathrm{i}$ percorsi per fissarne un profilo teoricamente spendibile/credibile, esse compiono in fondo una operazione rassicurante e pacificatrice, ma lo fanno a fatti avvenuti. Gli eventi clinici, invece, nella loro delicata tensione, chiedono di essere letti nel presente, hic et nunc, senza il tempo di rinvii a una doxa fondata.

Queste due proposizioni apodittiche - metodo come processo in movimento, modelli teorizzati solo a posteriori - esprimono invece la sostanza del tema del metodo clinico della tradizione classica italiana come io l'ho vissuto: senza eroismi, senza dottrina, senza retorica, senza guide. Una profonda delusione? Un simile scetticismo per le teorie era il segnale che il mondo in cui ci stavamo formando era debole in cultura $\mathrm{o}$ in capacità interpretativa? No, di certo. Una ulteriore, terza, risposta, sia pure soltanto marginale, sul come era fatto il metodo clinico classico ce la anticipava - provvisoria, forma larvale di pedagogia metodologica - il modo in cui la trattatistica medica esponeva i quadri di malattia . Nella scansione stessa dei paragrafi, ogni libro di patologia medica già suggeriva con onestà molti dei passaggi di metodo che compiamo nell'avvicinarci alla diagnosi e alla scelta di cura: la definizione, la eziologia, la patogenesi, la clinica, la diagnosi, la diagnosi differenziale, il decorso, la prognosi, la terapia. Oltre a comporre il profilo di malattia, questi elementi erano anche il primo fragile annuncio di un percorso diagnostico.

Anche i trattati del tempo suggeriscono qualche spunto interpretativo sulla genesi del metodo. La premessa è che studiavamo su trattati solo italiani. I libri italiani su cui si è formato il cosiddetto modello classico, pur ispirati idealmente a grandi personaggi del nostro passato (Fabrizio da Acquapendente, Morgagni, Spallanzani, Baccelli e giù sino a Murri o, nella tradizione più vicina, Frugoni o Campanacci), 
erano ancora concepiti, dall'inizio del Novecento, secondo la impostazione mentale della trattatistica tedesca (e, in misura minore, francese). Il carattere di fondo di questa trattatistica era quello di fornire riferimenti certi, saldi, sulla natura e la fenomenologia delle malattie. In altri termini, non solo l' approccio era totalmente centrato sulla malattia ma lo stampo era quello di definire, letteralmente nel senso di delimitare confini precisi alle malattie, di chiuderle. Ben oltre la fine della seconda guerra mondiale, a Germania soccombente, il modello compatto tedesco era ancora dominante. Le malattie erano come sigillate, al sicuro. Era con queste rigide sicurezze che ci si confrontava nel momento della scelta diagnostica. Questa concezione, ripensata dopo l'avvento e il dominio della logica didattica anglosassone, americana quantitativa, statistica, probabilistica, dubitativa, controllata - appare oggi quasi mostruosa; eppure essa è stata centrale anche nella formazione dei nostri Maestri (che passavano lunghi periodi di formazione in quei paesi europei). Era, anche per questo, una trattatistica piena di sindromi eponimiche, in cui vigeva poi una concezione proprietaria delle malattie: non solo ogni malattia era chiusa e ben recintata ma alla sua sicurezza vigilava un proprietario (Sindrome di ..., Malattia di ..., segno di ...) ben deciso a difenderla. Malattie compatte, insegnamento centrato sulla malattia, malattie di proprietà - erano i limiti clamorosi su cui si sono formate intere generazioni di medici, compresa la mia. Resta enigmatico come siamo riusciti a superarli e ad uscirne, cambiati dalla modernità.

Queste considerazioni vanno nel senso di molto relativizzare, per non dire di negare, che nella preparazione classica esistesse una entità concettuale autonoma definibile come metodo. Per questo, più modestamente, può essere utile provare a capire quali e come erano gli strumenti di diagnosi che costruirono, in modo artigianale, quello che vorrei poter chiamare metodo.

\section{Gli strumenti diagnostici determinano il metodo}

Il metodo non è neutrale, indipendente dalle circostanze. Anche il metodo clinico classico è stato dettato se non determinato (come lo è, e anche più vistosamente, il metodo di oggi) dalla quantità e dalla qualità degli strumenti diagnostici di cui si disponeva. Ripensiamo al passato. Immaginiamo, con banale metafora, che la soluzione diagnostica e la decisione di cura si regga, come un tavolo, su 4 gambe: la anamnesi, l'esame obbiettivo, la chimica-clinica di laboratorio, gli esami strumentali. Seguendo i cambiamenti di ognuno di questi 4 supporti si può provare a dar forma ad una parte della storia del metodo classico. Negli anni, dal 1955 - anno della mia laurea - ad oggi questi supporti hanno avuto ben diverso destino. La anamnesi, da estesa e strenua è divenuto oggetto minimo, in parte sostituita dalla sommaria interrogazione per problemi o dalla adozione di cartelle cliniche a domande preorganizzate. L'esame obbiettivo si è fatto sempre più breve, sovrastato se non cancellato dall'apporto della ecografia (e in taluni Atenei l'esame di Semeiotica Medica, nella sua parte di esame fisico del paziente, è stato abolito o è naufragato nel mare indefinito della fisiopatologia clinica). Diversa la sorte toccata alla altre 2 gambe. Con l'avvento di misurazioni automatiche, il laboratorio di chimica clinica è divenuto ricco di dati, spesso non richiesti, anzi ridondante (al punto da generare la diffusa angoscia degli asterischi che segnalano patologia). Gli esami strumentali poi, con l'affermarsi di un imaging strepitoso, hanno sovvertito la logica dei percorsi metodologici, accelerando in una misura un tempo imprevedibile il risultato diagnostico (e anche qui fornendo una quantità ingombrante di reperti incidentali, incidentalomi). In sintesi, le prime 2 gambe del tavolo si sono fatte esili e deboli, le ultime due solide o spregiudicate. Vedremo quali sono state le conseguenze di questo minaccioso squilibrio, del tavolo, sghembo, sempre a rischio di crollare. Per ora preme dire, con tutta l'enfasi possibile, che il cosiddetto metodo classico fondava quasi esclusivamente sui primi due supporti; la anamnesi e l'esame obiettivo.

\section{La anamnesi (e la re-anamnesi del $5^{\circ}$ giorno?)}

Esercitarsi a capire come si interroga un paziente era la prima prova cui il nostro mentore prescelto (speravamo bene) ci sottoponeva. Si trattava di un esercizio impegnativo, una indagine che sortiva spesso storie monumentali. Il compito era anche rischioso. La lettura in pubblico, durante la visita, sotto il controllo del paziente - primo garante della fedeltà -, dei coetanei studenti o giovani medici, del Medico caporeparto soprattutto, era un momento di forte esposizione. Rischioso perché - situazione oggi impensabile - poteva capitare di sentirsi irridere o, peggio, di vedersi strappare in Sala, coram populo, $\mathrm{i}$ fogli con la traccia provvisoria del racconto anamnestico faticosamente confezionato. Il trauma psichico era considerato formativo.

I contenuti del racconto andavano ben oltre la informazione canonica. Nella anamnesi familiare, la indagine sulle cause di morte si estendeva sino al lontano passato degli avi e dei collaterali. La anamnesi personale fisiologica era piena di richiami: la nascita, la scuola, l'allattamento, i primi atti di vita, l'età della scuola e della crisi puberale, i caratteri del ciclo femminile, il matrimonio, le gestazioni, la menopausa 
ecc. La fisiologica, poi, era un racconto a tutto tondo - oggi si direbbe multidimensionale - in cui c'era posto per dati sul lavoro, sul cibo, sui farmaci, i trasferimenti quotidiani, magari lo sport - allora rudimentale - gli affetti, le passioni, la politica. Ricordo che, nella personale remota, la attenzione maggiore era allora alla scarlattina e alla pertosse, la prima come spia di al tempo frequentissime - nefropatia o dei postumi cardiaci del reumatismo articolare, la pertosse, per eventualmente interpretare stati enfisematosi $o$ bronchiectasie, a presentazione, allora, scolastica, libresca.

Era però la anamnesi prossima il punto più insidioso e vulnerabile del racconto. Nella sua ricostruzione i sintomi più incerti erano, come oggi del resto, la febbre, il dolore, i disturbi del respiro, gli edemi, ecc. Tutti i dettagli erano presunti preziosi: tempi, modi, ritmicità, esordi e recidive dei sintomi, irradiazioni, rapporti con eventi fisiologici o patologici, eventuali associazioni di sintomi e loro cronologia. Un esempio: quello delle raccolta dei sintomi d'inizio di una cardiopatia congestizia: il malato crede di riferire esattamente la propria storia con il dire che l'edema agli arti inferiori è comparso improvvisamente, in pochi giorni, in condizioni di pieno benessere; solo dopo questa sua rappresentazione immediata, re-interrogato ammetterà la nicturia, la pesantezza epigastrica, la succulenza pretibiale serotina, in una parola tutto il corteo dei sintomi che preludono allo scompenso di cuore. Tutti questi dettagli erano accuratamente pesati dal Medico capo-reparto. Poteva capitare che al paziente - che nel frattempo si era familiarizzato con il reparto e aveva capito, a suo modo, che cosa poteva essere ancora utile riferire per la sua salute, un particolare che si rivelava poi importante venisse in mente solo qualche giorno dopo il ricovero e questo riorientava l'ipotesi diagnostica. Questi ritardi di racconto erano frequenti, al punto che il Medico caporeparto mio mentore si spinse a proporre di istituzionalizzare anche una reanamnesi del $5^{\circ}$ giorno dall'accesso. L'esperimento durò poche settimane, la proposta fu lasciata cadere, per i nostri pur rispettosi mugugni: sabotaggio concordato.

\section{L'esame obbiettivo (e la coralità della visita)}

L'esame obbiettivo aveva i tre modi tecnici che valgono tutt'oggi. Il primo, l'esplorazione corporale totale, nelle sue 4 espressioni obbligate, si svolgeva secondo la pratica storica, cominciando, chissà perché, dopo la minuziosa ispezione della pelle, dall'apparato linfoghiandolare, e proseguiva sino alla esplorazione completa del corpo del malato. Quando la ipotesi diagnostica era sufficientemente solida, l'esame obbiettivo - secondo modo - si focalizzava, seguendo quasi soltanto la oggettività che si presumeva interessata dalla malattia; era quello che oggi viene chiamato esame obbiettivo guidato dalla ipotesi. La terza modalità riprendeva questo modo selettivo di esame, cercando conferme o eventuali modificazioni della obbiettività ritenuta ormai centrale. Tutto questo veniva scritto in cartella, verificato ed eventualmente corretto dallo stesso Medico caporeparto. Quello che dava un senso speciale a questi passaggi era però la coralità della visita. Questa aveva, a ben pensarci, un aspetto esterno, rituale, ed uno serio, maieutico. La visita del mattino, almeno una volta la settimana, del Capo-in-testa, seguiva tempi teatrali: il pre-annuncio, la attesa dell'arrivo, il silenzio, e poi, cominciata la visita, i cauti lanci diagnostici dei comprimari, la verifica, una proposta (non sempre) demiurgica del Capo a conclusione di una rispettosa drammaturgia. Ben altrimenti fertile era la visita quotidiana, con il Medico Capo-reparto. Vi era ancora qualche traccia, dimessa, di esteriorità ma, a compenso, vi era molta sostanza didattica. La esperienza e la cultura di chi guidava il gioco era decisiva, altrettanto grandi erano però la attenzione e l'impegno di chi provava a misurarsi con la Semeiotica fondamentale. Il Medico Capo-reparto invitava a sentire un reperto paradigmatico, lo studente maturo o il giovane medico erano invitati a controllarlo. Nell'apprendimento di ognuno dei baluardi semeiologici c'era sempre una prima volta, un giorno di fondazione, non dimenticabile, mai dimenticato: il soffio anforico dei grandi versamenti pleurici o il rythme de rappel, reperto oggi dimenticato ma cruciale nella diagnosi di stenosi mitralica, allora comunissima e grave. Vi era anche un contenuto di sfida amicale tra noi discenti: tra chi aveva sentito giusto e chi in parte, e chi era rimasto comunque incerto. La visita era un momento di incomparabile ricchezza formativa. Anche più avanti negli anni, quando, maturati, siamo diventati noi stessi guida, la crescita in professione non finiva. Sentire con civiltà il parere di tutti e poi discuterne, rincorrendo ipotesi diagnostiche messe in campo con libertà e ragione, talvolta anche solo per essere confutate e costituire comunque futura memoria; e poi il piacere della osservazione intelligente che, anche se marginale, allargava il campo delle possibilità diagnostiche. Il potere educativo della esperienza confrontata e condivisa era irripetibile. Certo, oggi un qualunque amministratore fremerebbe all'idea di avere per 2 o 3 ore un primario (oggi, ahimè, Direttore!) e 4-5 assistenti insieme, mentre esercitano la propria destrezza clinica attorno a un letto e doverli pagare tutti per questo loro tirocinio (quasi al modo di una passeggiata, di una promenade: così, letteralmente, si esprimeva un Maestro, spiritoso e umanissimo, parlando della visita, indovinandovi in tralice una levitas piena di grazia).

Anche il Il dopo-visita, un po' simile alla mezz'ora del tea-time inglese, aveva sempre una sua utilità. Si tornava a parlare dei casi più problematici ma in modo 
più confidenziale e libero: l'intero reparto - assistenti, medici, studenti - erano ogni giorno in guardiola, attorno al Capo-reparto. Avviata da qualche battuta di sollievo, la conversazione ritornava sul malato, le idee per così dire si riaggiustavano. Si rivelava in modo ora informale ma preciso l'effetto di mentoring del caporeparto, in ognuno cresceva un poco la fiducia (oggi chiamata auto-stima).

\section{Significato e aspetti strategici di anamnesi ed esame obbiettivo}

In questa somma i due addendi avevano (hanno?) un peso diverso. L'esame obbiettivo (ed a maggior motivo poi le tecniche di laboratorio e strumentali) danno del paziente il quadro di una condizione del momento, di una fase in atto, di uno stato per definizione transitorio, come la istantanea di un evento in fieri. Dalla anamnesi emerge invece - nell'insieme di bagaglio ereditario, predisposizioni, abitudini, ambiente, reazione alle cariche emozionali - la espressione della personalità di fondo, cioè di un elemento statico, continuo, immagine del carattere e dei travagli di una vita. Non a caso, in una immaginaria graduatoria di sondaggi esplorativi tra persone, l'anamnesi è anche il primo contatto, il biglietto da visita scambiato tra paziente e medico. Per questo essa richiede, da parte del medico, doti - quali comprensione, intuito, analisi psicologica, capacità di sintesi, una dottrina profonda - che si affinano con la esperienza.

Prendeva così corpo, col tempo, una vera e propria tecnica della indagine anamnestica. La anamnesi doveva proporsi di tenere il filo nella spesso disordinata descrizione degli eventi. Per questo il paziente andava sorretto e in parte guidato, sia pure con discrezione e ritegno. In una prima fase gli si lasciava campo per una esposizione fluviale, libera, dei problemi; a questa, ascoltata in silenzio, seguiva la richiesta di una ricapitolazione che il medico proponeva per una specie di verifica; se accordata, ci si organizzava, ancora una volta insieme, per estrarre dal racconto, le priorità, le cose che il paziente sentiva come centrali. Senza questo percorso il paziente si sarebbe limitato agli episodi per lui salienti che magari aveva trascritto con diligenza in una auto-anamnesi. Il risultato di quella tecnica anamnestica era invece quello di estorcergli ricordi alla apparenza insignificanti e marginali. In un interrogatorio disciplinato Il paziente, rilassato, si sentiva aiutato e si dava forza per arrivare a definire bene i suoi problemi di fondo. Su tutto, il medico, alla fine, in risposta, suggeriva un suo parere provvisorio e le probabili soluzioni.

Il punto di forza è che, mentre tutto questo avveniva, in questo dialogo stretto e partecipe, nel racconto esteso al dettaglio più minuto, nasceva tra $\mathrm{i}$ due interpreti una intesa virtuosa: si erodeva un po' di asimmetria culturale e nel paziente si affacciava la pur fragile coscienza di poter essere parte della decisione. Nasceva una buona premessa di compliance (ma allora la parola non si usava). Così, con la anamnesi .

L'esame obbiettivo poi, nella confidenza con la fisicità del corpi - quanta inarrivabile comunicazione in quegli atti del vedere, toccare, sfiorare nella percussione, ausculatare il corpo del paziente!! rafforzava e sanciva definitivamente l'intesa. Alla fine la condizione che si creava prendeva un significato ben diverso da quello dei lemmi che si usano, e spesso a sproposito, per definire il rapporto con il paziente. Non era in ballo, per intenderci, la umanizzazione nel senso in cui è stata oggi come istituzionalizzata: predicata, insegnata, persino programmata nei corsi di formazione, quasi si trattasse di un atteggiamento aggiuntivo di galateo delle buone maniere verso il paziente. Quella prima conoscenza era invece un atto intrinseco, costitutivo del rapporto con il paziente, una alleanza, sia pure non tra pari. In alcuni casi estremi le sue conseguenze erano addirittura mostruose, sino a rovesciare un risultato che all'inizio era sembrato positivo. Poteva accadere: il paziente si liberava del suo carico di malattia e lo riversava sul medico; il medico - oggi diremmo con molta incoscienza - se ne caricava quasi corporalmente, come fagocitando i problemi del paziente, e premiandosi in una confusa ebbrezza di potere tecnico. La decisione ridiventava monocratica, il passaggio di responsabilità era una vera e propria delega che il paziente dava al curante. Tuttavia, anche in questi casi non bilanciati, nel sistema per sua natura formale del colloquio si inseriva come una realtà intensificata e sfocata, come una inattesa vibrazione, una tenue ventata di mistero. Anamnesi ed esame obbiettivo erano il segno più riuscito di quella inesorabilità dell'ascolto reciproco che io interpreto non come momento consolatorio o di dovuta pietas ma come atto squisitamente tecnico.

$\mathrm{Al}$ di fuori della proprietà delle conclusioni e al di là di queste, il modus di questi due processi dava quasi sempre il risultato di raggiungere il traguardo olistico, il luogo mentale cioè in cui i problemi, i problemi del corpo malato e la sfera psichica del paziente, si ritrovano magicamente indistricabili, inscindibili. Era lo stato che i filosofi chiamano sublimazione, uno spostamento favorevole della meta: ${ }^{5}$ da soltanto pratica - il fine utilitaristico di raggiungere comunque una diagnosi con la povertà degli strumenti a disposizione - ad un traguardo più lontano e più alto, all'inizio impensabile.

Ci ritrovavamo così, alla fine, alla Murri, ${ }^{6}$ medici della persona dopo avere seguito, forse inconsapevolmente, di Murri, la radicalità del metodo induttivo. Ci mancava solo il suo afflato di amore universale per la umanità. Lo testimoniavamo, più 
umilmente, con la adesione, quasi una complicità fisica, ai problemi del paziente.

\section{La matrice filosofica...}

\section{...della fase della acquisizione da elementi percepibili (anamnesi, esame obbiettivo): reversibilità e sinergia di induzione e deduzione}

Il modo disordinato del nostro buttarci sul paziente, una volta approdati in Reparto dopo i 3 anni propedeutici, anticipava in forma grezza quello che poi sarebbe stato consacrato come il già citato learning by doing, si impara facendo, ripresa tardiva di Gianbattista $V_{i c o}^{7}$ per il quale l'uomo conosce in senso completo soltanto ciò che fa, non la natura dunque ma la storia. Il fondamento di questa certezza sta, in eccentrica polemica con il razionalismo scientifico cartesiano, nell'assumere il fatto, ossia ciò che si compie o si produce, come vero. Vico affermava, con sconcertante modernità, che gli uomini dapprima sentono senza avvertire, poi avvertono con animo perturbato ... finalmente riflettono con mente pura.

Questo modo di pensare oltre cha da vecchie riflessioni, sempre confermate, mi deriva dalla ricordata insofferenza istintiva per la concettualizzazione metafisica e, per inevitabile converso, dalla forte simpatia per il pensiero empirico. Per i filosofi inglesi del Seicento e del Settecento David Hume in testa a tutti - le nostre conoscenze non vengono dai concetti, come sosteneva in termini opposti ed estremi Leibniz, bensì dalla esperienza sensibile che poi si stratifica grazie alla abitudine e al ragionamento. ${ }^{8}$ Partendo dalla nostra tabula rasa, dice Hume, abbiamo costruito un nostro personalissimo modo di incedere mentale, i concetti sono soltanto un modo veloce e spesso ingannevole per codificare la esperienza. Questo gli empiristi. È però vero che, pur non conoscendo attraverso le idee generali leibniziane, anche la esperienza, in particolare proprio quella che si accumula nel procedimento diagnostico e decisionale, richiede certezze. Il passaggio dalle sensazioni particolari alle idee generali richiede dunque un controllo, perché le prime non siano solo impressioni. Per dirla in altro modo, la nostra esperienza osservativa, pur essendo la base di ogni conoscenza, è un fondamento insufficiente perché, induttiva, è solo probabile. Nella decisione diagnostica non ci basta dunque un pur solido empirismo. La verità era che avevamo anche noi, come guida acquisita, un nostro personale (ahimè leibniziano) $a$ priori. Anzi, era augurabile che esso fosse altrettanto ben fondato, esteso, internalizzato, nostro. Era quell' $a$ priori che ciascuno di noi aveva messo insieme con lo studio appassionato della dottrina medica e che ci era stato consegnato nei trattati dei Maestri. La combinazione dei due ingredienti - il riferimento empiristico alla esperienza e alla psicologia da una parte, il richiamo all' a priori e alla essenza deduttiva della dottrina dall'altra - erano il punto d'inizio nella conduzione del processo diagnostico.

È interessante valutare questa interpretazione scolastica del metodo classico della tradizione italiana con il metro del suo testimone più illustre - e più citato - del dopo-Unità d' Italia , tra i clinici, ancora Augusto Murri. Il focus, teorico ed umano, della visione di Murri è l'esame del singolo malato, assumendo la sua centralità come premessa . Lo strumento privilegiato per raggiungerlo è lo spirito di osservazione e il ricorso alla anamnesi, gli strumenti che caratterizzano il metodo induttivo. Murri è indicato come l'assertore primo di questo metodo i cui strumenti consentono di evitare la argomentazione deduttiva, ${ }^{9}$ prefabbricata e paludata ma sempre accessibile, con cui la esperienza si confronti e si corregga. Va osservato però che Murri stesso ha in mente un medico che sia anche di formidabile dottrina, forte delle nozioni apprese nella scuola. Per capire e dare sicurezza alla propria esperienza questo medico può fare sempre ricorso alla nozionistica fondata dai Maestri e consegnata nei trattati. Questa dottrina altro non è - lo si ripete ancora una volta - che il sostanziale a priori che permette di ordinare l'esperienza, mettendola a confronto con un campione credibile di disciplina fondata. In altre parole, anche in Murri, come nel metodo classico, il procedere delle ipotesi e delle decisioni conseguenti si conferma come un continuo vicendevole stimolarsi e inseguirsi di momenti induttivi e deduttivi che, più che contrapporsi, sono sinergici. Anche se intimamente costituito e permeato dal valore primario della osservazione del paziente, il metodo classico tradizionale (e la mia personale e sopravvenuta convinzione) ripropone la storica dialettica HumeKant sul tema della conoscenza. Hume aveva fatto dipendere la scienza dalla esperienza concludendo che la scienza è solo probabile. Kant, pur insofferente dell'estremismo leibniziano sulle idee precostituite, aveva capovolto la prospettiva, basando la certezza e la necessarietà della esperienza sul fatto che essa è fondata a priori dalla scienza. Di fatto scienza ed esperienza costituiscono - altro mio momento eretico - due poli tra cui intercorre una reversibilità completa. Ferraris, ${ }^{8}$ un po' dissacrando, sostiene che la scienza è una esperienza un po' più raffinata e sistematica $\mathrm{e}$ che l'esperienza è una scienza un po' più alla buona $\mathrm{e}$ alla portata di tutti.

La reversibilità e sinergia di induzione e deduzione è sottolineata ancora più incisivamente dal pensiero filosofico degli ultimi anni anche se esso sembra cambiare il peso rispettivo del contributo che le due componenti, induttiva e deduttiva, dànno alla 
diagnosi. Per Karl Popper il senso della matrice filosofica del conoscere è che nella scienza non basta osservare ma bisogna sapere anche cosa osservare: la osservazione non è mai neutrale (e qui viene in mente Heisenberg) ma è sempre intrisa, più o meno consapevolmente, di teoria . Per Popper, in totale contrasto con Vico, la teoria precede sempre la osservazione: anche in un approccio presunto empirico e induttivo la mente umana tende sempre a sovrapporre i propri schemi mentali alle scelte osservate. Dunque, secondo Popper, quella che noi interpretiamo come la induzione - anche quella di Murri - sarebbe in realtà una deduzione perché costruita sempre (con un) a priori. Se mai, per Popper, la induzione è il limite negativo della deduzione, nel senso che essa serve non a costruire (conferme) ma se mai a demolire (confutazioni) gli assiomi generali (generati).

\section{...della fase del ragionamento decisionale: la invarianza dei processi mentali coinvolti}

Il secondo problema conoscitivo riguarda il modo e i percorsi mentali ai quali venivano avviate le conoscenze generate da anamnesi ed esame obiettivo, nell'intento di favorire o determinare la decisione. Anche in questo ambito la tradizione classica italiana ha adottato e praticato un eclettismo che ricorda da vicino quello messo in atto nella fase osservativa, della acquisizione dei dati. La pre-condizione, scontata, era che come ogni persona normalmente dotata e non diversamente da essa, il medico fosse provvisto di logica. Questo non significa che egli dovesse necessariamente conoscere elementi di logica, come dottrina autonoma, separata, ma che sapesse, come in ogni altro momento di vita, ragionare. La domanda è dunque se, nel seguire questi percorsi, il metodo della medicina classica, della tradizione italiana, si caratterizzasse per meccanismi originali che ne rendessero singolare la logica mentale. Una risposta, ovvia, ripetuta, è che il ragionamento era guidato dalle circostanze, personali del medico e storiche, del contesto di cultura . In ogni caso - e questo è un altro punto nodale - gli itinerari del ragionare sono indefinibili per loro natura, da sempre; tali erano, tali sono ora e saranno in futuro, per quanti sforzi le neuroscienze possano produrre nel tentativo di categorizzarli. Per questo, alla questione di quali motivazioni prima innescano e poi indirizzano il ragionamento clinico in una direzione data o analoga o simile o, al contrario, verso la strada opposta, la risposta elementare è che è molto improbabile che la mente abbia cambiato nel tempo la sua tecnica di lavoro, il suo modo di procedere . Tornano a proposito alcune considerazioni generali.

La prima: Nell'ultimo mezzo secolo, la mente del medico si è arricchita di nuove conoscenze, di facilitazioni inattese, consentendogli diagnosi sempre più circoscritte e raffinate. Questa molteplicità di possibili è stata ulteriormente alimentata $\mathrm{o}$, se si vuole, appesantita, dal proliferare, nell'alveo della stretta madre medica, di un gran numero di culture subalterne specialistiche. Si sono così creati setting di lavoro in gran parte nuovi e questi hanno moltiplicato gli approcci pratici ma anche e soprattutto mentali del ragionamento. La quantità di acquisizioni che si è generata è stata messa in ordine, sin dove possibile, attraverso modelli che esemplificano la varietà delle situazioni di contesto generale e di cultura personale del medico ragionante. Gnerre e Nardi, ${ }^{10}$ in questa sede, si sono posti il compito gravoso di descriverne il substrato logico, di elencarne e ordinarne la varietà, di disciplinarlo in modo che ne sfugga la minor quantità possibile di verità operativa. Così, partendo dal confronto dei due modelli di approccio di base quello intuitivo euristico e quello deduttivo analitico ipotetico - vengono indicati i diversi strumenti di ragionamento clinico utilizzati nel processo decisionale: la pattern recognition, le linee-guida, le flow-charts, gli algoritmi, il modello event-driven, lo scartare lo scenario peggiore, ecc. Applicando questi strumenti alle condizioni concrete di lavoro clinico, essi ne confrontano il diverso rendimento in termini di rapidità: strategie rapide: auto-diagnosi, colpo d'occhio, trigger di riconoscimento; strategie lente: esclusione limitata di diagnosi possibili, definizione graduale della diagnosi, ragionamento probabilistico - e di prossimità alla diagnosi. Che dire? Personale giudizio è che, pur analitica e puntigliosamente curata, la esemplificazione non è, e non potrebbe esserlo, esaustiva. I percorsi di ragione che, anche nel solo ambito della medicina come disciplina, la mente, messa alla prova del decidere, ha a disposizione, sono senza numero. È perciò inevitabile che alcuni di essi, pochi o molti che siano, sfuggano ad ogni pur diligente descrizione. A questo tentativo di disciplina sfuggiva dunque anche il metodo clinico antico, nel suo eclettismo.

Più analiticamente, una strutturazione ordinata del ragionamento medico fa porre almeno tre ordini di riserve critiche: i modelli elencati sono provvisori, dedotti a posteriori, non insegnabili. Quanto al primo punto, tutto fa prevedere che, con il cambio vorticoso della pratica materiale medica che si annuncia, anche questi che oggi si presentano come verosimili siano in realtà modelli fragili, esposti ad un rapido mutamento e destinati ad ulteriormente moltiplicarsi e intricarsi. Ancora, questi modelli sono stati dedotti da verifiche successive al momento della diagnosi, ripensando su come deve essersi atteggiata le mente in quella particolare circostanza, dunque, ancora una volta, a fatti clinici avvenuti, sono cioè risultato di considerazioni a posteriori. Proprio in ragione di 
questo, infine, essi hanno scarsa probabilità di essere insegnati: la sintesi loro costitutiva, processo per eccellenza deduttivo, è il risultato di definizioni, postulati, assiomi e problemi ineccepibili ma, per dirla con Cartesio non dà... soddisfazione agli spiriti di quelli che desiderano di imparare, perché non insegna il metodo col quale la cosa è stata trovata. ${ }^{11} \mathrm{Si}$ tratta cioè di una acquisizione sterile sul piano, come si dice, operativo.

Va osservato poi, in proposito, che le acquisizioni più recenti di neurofisiologia, meglio di neuroscienze, su funzionamento della mente ed economia del lavoro cerebrale, ci forniscono una riflessione ancora più indisciplinata e corrosiva. Mi riferisco alla nozione della non linearità dei procedimenti mentali. ${ }^{12} \mathrm{Nel}$ suo lavoro la mente non segue un percorso lineare. Anche se spesso costretta a procedere in modo lineare, essa è probabilmente pensata e creata per muoversi, stimolata, secondo una infinita probabilità di connessioni non prevedibili. Non costretta, essa divaga, raccoglie e manda stimoli, si distrae accendendo nuove scintille, apre canali inesplorati, è sviata da nuovi stimoli, subentrati senza un motivo e generatori essi stessi di nuovi flussi di pensiero, scollegati, fuori tema. Per dirla dantescamente, la mente si immilla. $^{13}$ Del resto funziona così, icasticamente, la mente del bambino che crea legami liberi e volatili e che, se chiamata ad esprimerli, lo fa attraverso una varietà inedita di linguaggi - i cento linguaggi di Malaguzzi. ${ }^{14,15}$ È poi interessante osservare che il link digitale del web, con i suoi infiniti rimandi, replica questo funzionamento libero della mente, mostrandosi, specularmente, come la controparte tecnologica di strade che la mente percorre in modo istintivo, senza una mappa affidabile che non sia la pura ragione e la coscienza. Così, appunto, si muoveva il ragionamento clinico della classica tradizione italiana, con una mirabile libertà e con un unico limite: la solidità delle conoscenze dottrinali duramente compitate e apprese. In forza di questa riserva estesa di libertà esso affrontava e spesso risolveva i suoi problemi, guidato solo, deduttivamente, dalla tenuta di quelle conoscenze di dottrina. Infatti, prima, nella fase dell'apprendimento, dello studio disciplinato, la mente era stata costretta alla linearità, richiamata a non improvvisare, ad imparare dati certi, fondati, nati dalla osservazione e dalla riflessione dei Maestri.

Per questo, ritornando al tema dei percorsi mentali alla diagnosi ${ }^{10}$ lo sforzo attuale di disciplinarizzare il ragionamento clinico, di vederlo come variabilità precostituita di itinerari mentali noti, costituisce un interessante esercizio speculativo ma non si adatta al senso cognitivo stretto, per così dire normalizzato, che si deve alla parola metodo, non ne ha la forza e il disegno.
In definitiva, e per ripetermi, da allora, dai tempi classici ad oggi, nulla è cambiato quanto a (eventuale) definizione della fisiologia e della filosofia dei percorsi mentali impiegati nella diagnosi.

Unica differenza è, se mai, che la mente del medico classico si muoveva in una materia complessiva in certo senso contradditoria Da una parte i trattati davano una elencazione descrittiva di sintomi o di segni che era certamente puntigliosa ma affollata e imprendibile, di tipo fondamentalmente qualitativo, senza cioè alcun orientamento - a parte i cosiddetti, e scomparsi, sintomi patoiognomonici - sulle loro prevalenze e sul loro possibile diverso peso nella decisione finale; non fornivano insomma alcuna guida (appunto: probabilistica) rassicurante. Nel verso contrario, la medicina classica si mostrava rigida sul piano nosografico, lontana cioè dal modo in cui oggi intendiamo la realtà di patologia, cioè come una condizione spesso senza limiti netti tra salute e malattia e governata solo dal problema della distanza da una o dall'altra delle due condizioni; è questa distanza che dà la misura della probabilità che i dati clinici siano effettiva espressione di una specifica condizione morbosa.

In definitiva, sembra problematico riuscire ad insegnare ai medici una strada mentale per arrivare alla diagnosi. Forse, tra quelli descritti da Gnerre e Nardi, il solo profilo di percorso che si può utilitaristicamente proporre in anticipo e perciò trasmettere con umiltà, e insegnare a prescindere, in modo meccanico, poco ragionato ma diagnosticamente redditizio, senza troppo filosofare, è quello di escludere lo scenario clinico peggiore (rule out worst-case scenario), tenendo bene in mente al momento della diagnosi, e ripeterseli come una giaculatoria, i cosiddetti big five (dissecazione aortica, infarto miocardico, embolia polmonare massiva, pneumotorace iperteso, sindrome di Boerhaave) per esorcizzare gli errori diagnostici più rischiosi (o sventolando le red flags).

Alla fine, sul significato del metodo nel ragionamento clinico, sembra illuminante una considerazione di Cesare Scandellari. ${ }^{16}$ Attraverso il racconto della evoluzione del modo di fare il medico, Scandellari riprende, parafrasando Murri, il carattere della diagnosi come attività e dunque come ricerca. Questo gli dà modo di esaminare le analogie tra ricerca medica e ricerca scientifica. Dopo averne indicato gli elementi di contiguità egli, con la onestà intellettuale che gli è propria, (ma con malcelato sottofondo terroristico, dissuasivo) elenca gli aspetti distintivi del metodo scientifico sensu strctiori: limitatezza delle ipotesi possibili, plausibilità delle ipotesi, probabilità delle ipotesi, possibilità di accettazione delle ipotesi mediante conferme, equivalenza pratica delle conclusioni ottenute per confutazione con le conclusioni ottenute per 
conferme, affidabilità delle conclusioni, ecc. Sulla base di queste constatazioni egli conclude che tra i due metodi esistono differenze, di forma - e tali sino al punto da rendere fragile per il procedere clinico la stessa designazione di metodo - ma anche di sostanza.

La differenza di sostanza tra le due attività (Murri), dello scienziato e del medico, è che tra il medico e il paziente è in atto un patto etico, una religio nell'etimo primo di legame, alleanza - che sovrasta e silenzia ogni questione di metodo. La fedeltà a questo patto è costitutiva della attività del medico e dunque non distingue il medico di oggi da quello della tradizione classica italiana. Si tratta di una clausola, che è del tutto estranea alla strategia e alla dinamica della ricerca scientifica. La scienza, dice Gianni Vattimo, che pure contribuisce a creare delle condizioni obbiettive che migliorano la vita dell'uomo, non è interessata alla sua felicità.

\section{Le peculiarità}

\section{Quello che mancava al metodo classico}

Rivisitato oggi il metodo classico si rivela povero e scalzo. ${ }^{17}$

i) Mancava la statistica: La statistica medica segna l'ingresso inatteso della quantificazione in Medicina. In Italia nasce come estensione all'uomo dei criteri di osservazione e misura adottati in microbiologia. Sono microbiologi i primi medici mi vengono in mente Deotto e poi il grande Maccacaro - che scrivono di statistica per i clinici da poco dopo la fine della seconda guerra mondiale. ${ }^{18,19}$ Sulla statistica il metodo clinico classico, qualitativo e intuitivo e spesso fallace, è dapprima sospettoso ma presto ne è sconvolto e ne sarà alla fine bonificato. La statistica medica ci ha insegnato, più che a confermare nostre congetture, a cancellare nostre certezze; spesso, più semplicemente, ci ha educato ad un esercizio del dubbio ancora più strenuo.

ii) Il medico classico neppure lontanamente sospettava di poter essere implicato (e rispondere) in temi di economia sanitaria. Questa è entrata molto faticosamente nella consapevolezza - e nella accettazione (la benigna voluntade dantesca) dei medici. La adozione, contro voglia, dei DRGs (diagnostic related groups) e, nei primi anni ' 90 , l'irrompere della politica della qualità furono passaggi traumatici. Al Medico si chiedeva - e poi si impose - il coinvolgimento gestionale ossia la responsabilità nella conduzione economica dei Reparti. Il totem di questa promozione era la stesura del budget. Ricordi sofferti e sgradevoli. $\mathrm{Ci}$ si cimentava, a proprio rischio, con una materia nella quale non avevamo categorie di orientamento e verso cui non provavamo alcuna curiosità intellettuale. Quanto fosse radicale e totale il passo era ben significato dal nuovo lessico - il pazientecliente, la gestione per processi, il prodotto, gli eventi-sentinella, le non-conformità forzosamente introdotto come segnale un po' sinistro di una nuova cultura. Solo molto più tardi (parlo per me) abbiamo capito che, con la politica della qualità, era offerto alla nostra personalità professionale, scarsa o buona che fosse, la occasione per aggiungere il secondo decisivo segmento della nostra dimensione civile.

iii) Mancava al metodo classico la possibilità di orientarsi sulla informazione scientifica e sulla sua qualità. Le riviste italiane di Medicina erano poche e poco diffuse. Solo alcune avevano referees. Era ignota la sofferenza di doversi difendere e replicare ai peer reviewer, il temuto andirivieni di correzioni e di aggiustamenti cui siamo ora ben addestrati. La editoria era quasi esangue il suo livello, misurato con il metro attuale, modesto. Abbonarsi alle riviste storiche europee era sporadico ed elitario. Poi, l'avvento della EBM, evidence-based medicine, ha scoperto molte verità. Definire livelli di evidenza e gradi di raccomandazione delle prove emerse dagli studi controllati era, prima, impensabile. Anche il solo proporsi di farlo.

iv) Altrettanto sconosciuta era la confidenza con le regole della sperimentazione clinica. Definire obbiettivi di una ricerca, prendere la misura giusta ai reclutamenti, bilanciarli, avere le idee chiare sulla fattibilità delle proposte avanzate, valutare risultati non solo sui dati obbiettivi ma anche su quelli giudicati in positivo dal paziente, - PROs, patient-related outcomes, ${ }^{20}$ trarre conclusioni oneste, senza enfasi. La varietà e il diverso valore clinico dei modelli di sperimentazione impiegati (randomizzati, controllati in doppio cieco, studi caso-controllo, di coorte, osservazionali, semplici case reports, ecc.) sono arrivati molto dopo.

v) Più in generale, in questo stesso contesto, era lontana la ambizione di potersi misurare con il mondo della ricerca. L'avvicinamento a questo mondo, che avvertivamo pieno di fasti (quanto rispetto evocavano quei primi nomi degli autori degli articoli!!), era segnato da un mix di inadeguatezza e cautele e da una debole spinta a rischiare. Solo molto tardi - i primi lavori pubblicati, l'accesso a riviste via via più qualificate - si arrivava a percepire la ricerca come un piacere, e non vanamente intellettuale, come profonda gratificazione e ripagamento. La ricerca era una forma inconscia ma efficace di monetizzazione immateriale, un formidabile antidoto alla stanchezza e ripetitività della routine (quello che oggi viene definito burn-out). 
vi) Si potrebbe pensare, a questo punto, che, già gravato dalle pesanti carenze appena elencate, il metodo cosiddetto classico fosse anche spoglio e dunque bisognevole, per darsi senso, di tutte le componenti che si indicano oggi come relazionali. Mi riferisco, in sintesi, alle valenze oggi declinate come etiche, bioetiche, antropologiche, sociologiche, di psicologia clinica, di scienza della comunicazione, di pedagogia medica, ecc.. Per fermare (ancora, dantescamente, fermalvi dentro) in modo organico i contenuti di queste discipline, negli attuali ordinamenti didattici di molte Università sono stati predisposti dei Corsi integrati con seminari comprensivi del loro intreccio. Queste competenze, va da sé, si presuppongono esterne, sovrapposte a quelle mediche strettamente professionalizzanti, ma al pari di queste altrettanto insegnabili, in corsi di didattica organizzata. Bene, il metodo classico, non aveva nulla di tutto ciò. Per sua fortuna e destino. Non ne aveva bisogno. Il livello di moralità (etica) e di complicità (socializzazione, comunicazione) espresso con gli strumenti della anamnesi e dell'esame obbiettivo raggiungevano - proprio perché inscindibili dal contesto tecnico patologico - un livello di verità umana (psicologia clinica) non paragonabile a quello che, spesso in maniera artificiosa e prefigurata forniscono le discipline speciali a ciò deputate. Queste trasformano il colloquio tra due figure dialoganti in un incontro regolato da schemi di dottrina autonoma a destinazione sistemica ma a genesi, lo si ripeta bene, extra medica; un incontro privo del substrato forte delle conoscenze mediche e della sua capacità di rispondere, in armonia olistica, a tutti i bisogni del pazientepersona. Detto in altro modo, la irruzione degli strumenti della società della comunicazione - cui queste discipline in diversa misura ma univocamente afferiscono - rinvia la infinita varietà e complessità della condizione umana e la sua dignità concreta a strutturazioni schematiche e generali di modelli e di percorsi mentali predefiniti, mortificandola (ל̀ molto semplice dice l'opinionista di comunicazione in - frequente, reiterato - collegamento $\mathrm{TV}$, e fornisce subito la soluzione, effettivamente molto semplice, alle questioni più labirintiche ${ }^{21}$ ). Era invece proprio in forza della chiamata ad una totalità complicata e misteriosa che la medicina classica, si era conquistata, anche, il connotato di arte. Ora, l'avvento di un Evo Elementare di tweet liberatori e risolutivi ne potrebbe cancellare il profilo.

Altra cosa, e meritoria, è lo sforzo di un clinico ${ }^{22}$ di analizzare retrospettivamente e di chiarirsi il cammino relazionale che lo ha portato al risultato del bene del paziente. Egli sa ben distinguervi un primo passo, cognitivo, di capire bene i problemi del malato; un secondo, affettivo, che nasce dal condividerne le esperienze, l'essere, come si dice, nei panni degli altri; un ultimo, più sottile e sublime (vi si ritorna!!), quello motivazionale, che porta, attraverso la compassione, al bisogno urgente di fare qualcosa per lui. Questo, sì, rappresenta una ricostruzione organizzata dei modi di quella che anni fa ho definito Medicina dell'ascolto. ${ }^{23,24}$

\section{Quello che non c'era nel metodo classico, ovvero un mondo della medicina estraneo alla connessione con il paziente}

i) Non c'era il paziente virtuale, il paziente come icona, l'icona come paziente, l'I-patient'. ${ }^{25} \mathrm{Si}$ capisce lo sgomento di un giovane medico che per il tirocinio si aspettava di incontrare un malato, di parlargli, toccarlo, ascoltarlo ecc., ma che si ritrova invece in un semi-interrato tra una selva di PC dove vede il paziente sul monitor; il paziente come icona, la icona come paziente. Le belle sentenze che ha sentito nel corso degli studi - il nostro testo è il corpo del paziente o la mappa non è il territorio - sono amari richiami; il paziente viene osservato attraverso gli EMR, electronical medical records, che ne sono il surrogato. L'I-patient produce cartoma, non risveglia emozioni. ${ }^{25}$

ii) La impersonalità non si limita alla didattica ma si estende ora, nel medico formato, al momento finale del suo lavoro, quello della diagnosi e quello della cura. In tempi remoti in Clinica si additava all'ignominia il collega che, refrattario a toccare il malato, faceva la visita alle cartelle. Oggi le sequenze materiali di avvicinamento al malato sono in crisi. Le visite del mattino sono atti solitari $^{26}$ e muti in cui si interroga il monitor, magari con il paziente che chiede con gli occhi di essere ascoltato. La terapia segue lo stesso destino, la virtual care è dominante, si svolge davanti al computer, senza dover dare troppe spiegazioni sul perché si propone che cosa. Alla fine, nel lavoro di tavolino, il medico si dispone ad una fase, ancora solitaria, ${ }^{27}$ di videoscrittura. Il passaggio dalla interazione di routine con pazienti e colleghi ad una attività isolata e individuale è, così, completato. Di certo essa contribuisce alla (studiatissima) solitudine del medico, a dare forma, nel tempo, alla figura del medico infelice..$^{28}$

iii) Non c'era la tentazione della ipertrofia del ramo satellite. La attuale ridondanza di reperti accessori, soprattutto di chimica clinica, che di tanto in tanto mostra qualche anomalia - spesso solo marginale - spinge il medico incauto a seguire quella traccia, appunto il ramo satellite, che per lui è interessante $\mathrm{o}$ in cui è teoricamente versato trascurando il tronco del problema di fondo del malato: un pericoloso smarrimento delle priorità. 
iv) Non c'era la nobilitazione dei fattori di rischio elevati a malattie (ipertensione arteriosa, indici lipidici, ecc.) con la deriva invitante ad una medicina semplificata e intellettualmente pigra. ${ }^{29}$ La epidemiologia disastrosa di queste situazioni correlate ai fattori di rischio sospinge, da dentro la medicina esercitata, il moloch della amplificazione mediatica, che la sostiene e valorizza, creando confusione di metodo.

v) Non c'era l'overtreatment, l'eccesso di diagnosi e di trattamenti. Lo impediva il pauperismo tecnologico. Le risorse diagnostiche sono oggi frequentemente sovrautilizzate, ${ }^{29}$ spesso semplicemente per accertare la adesione alla terapia più che per il suo monitoraggio. Lo stesso si dica per l'eccesso di trattamento. L'esempio classico è quello dal cancro tiroideo per il quale la esperienza coreana è la più impressionante. La pratica dell'over-treatment è talmente estesa che alcuni pazienti, per colpevole contrappasso, se asintomatici, decidono consapevolmente di addirittura rinunciare a curarsi. ${ }^{30}$

vi) Non c'erano soprattutto - ma di questo si è già fatto parola - gli obbiettivi istituzionali - poi degradati ad aziendali -, dettati dalla scarsità di risorse e dalla applicazione sempre (giustamente) più rigida della politica della qualità. Gli obbiettivi economici, spesso tanto gravosi da essere anteposti al tema primario della cura del malato, accentuano oggi il dualismo della polarizzazione clinici/ amministratori. Effetto negativo è che anche il medico si dispone per acquisire sempre più delle qualità metriche, delle qualità quantitative altre, rubando sul tempo dedicato al paziente. ${ }^{27,31}$

vii) La esigenza, di seguire rigorosamente i tempi (durata della degenza, della visita, dei controlli ambulatoriali, ecc.) solleva un'altra difformità dal passato. Non c'era il limite assillante del tempo. ${ }^{31}$ Non avevamo di certo tutto quello, aperto, a disposizione degli scienziati che possono permettersi di arrivare a conclusioni transitorie e fallibili. Ne avevamo comunque molto. Ci concedevamo dei veri e propri lussi metodologici. Era comune stare ad osservare per giorni, a funzioni vitali rispettate, quale era il l'andamento di un sintomo che sembrava promettente in senso diagnostico. Questi lussi erano ben ripagati sul piano del metodo. A diagnosi finalmente conquistata, riuscivamo spesso ad enucleare, a posteriori, il sintomo/segno cosiddetto patognomonico di quella condizione di malattia. Anche il concetto di decorso, ora tristemente obsoleto, era ben coltivato. Nei casi più fortunati, dal decorso di malattie che vedevamo per le prime volte (allora: la polmonite primaria atipica, le crioglobulinemie, la connettivite mista, ecc.) riuscivamo a mettere insieme estetismo nosografico - abbozzi di storie naturali delle malattie.

\section{Effetti collaterali dell'abbandono del metodo classico: la medicina alternativa}

La rottura del rapporto medico-paziente, il suo graduale e quasi inavvertito abbandono, ha avuto l'effetto di lasciarne il patrimonio a personaggi furbeschi (e, in casi estremi, documentatamente fuori legge) che però hanno colto d'intuito, del rapporto, il valore e la insostituibilità. Il riferimento, si intende, è alla Medicina alternativa. Nella pratica della maggior parte delle cosiddette medicine complementari il dialogo con il paziente ha un ruolo centrale. Nelle più praticate esso è addirittura il caposaldo della presunta terapia. Il dialogo viene praticato in un desolato deserto di cultura, partendo però dalla elementare consapevolezza che il paziente reclama, con insistenza, ascolto e attenzione ai suoi bisogni. Le caratteristiche della medicina alternativa, a ben pensarci, vanno proprio in questa direzione. I suoi presupposti di fondo sono, sommariamente, tre. Primo, essa è, lo si è detto, scientificamente inesistente o si sostiene, avventurosa, su bizzarrie, stravaganze e fantasiose evasioni dal senso comune (l'anima dell'acqua, le molecole della omeopatia). Queste sono portate a fondamento di una anti-scienza che pretende cionondimeno - e questo è curioso - riconoscimento di dignità di cultura. Secondo, essa pratica l'ascolto del paziente in modo intensivo quando non esclusivo, e lo pratica bene, con fortuna, diremmo, e con molto successo, con buoni risultati. Terzo, essa ha avuto la intelligenza, o meglio la astuzia, di scegliere come campo d'azione preminente le condizioni (non direi le malattie) nel solo domain della soggettività. Si tratta di un domain immenso che della clinica raccoglie tutto il non misurabile, il non oggettivabile e quindi non valutabile comparativamente. Vi hanno posto condizioni mal definite come la dolorabilità in tutto il suo ipotetico range (mi dica quant'è il dolore, con un numero da 1 a 10), il senso di malessere, la piccola dispepsia, la stanchezza, la sensazione di febbricola, la nebulosa e codificata sindrome da fatica cronica, la fibromialgia, la ipocondria da conversione viscerale, sino all'angoscia immotivata, e giù giù sino ai segni di disagio sociale, i cosiddetti bisogni indifferenziati. Ora, il metodo classico, con i suoi soli strumenti antichi, può (deve) riprendersi anche il territorio smisurato della soggettività, e riqualificarlo.

\section{Il ritorno, attualizzato, del metodo classico: la medicina dell'ascolto o medicina interpersonale}

La Medicina basata sulle prove di efficacia, $\mathrm{EBM},{ }^{32}$ ma non solo essa, tutto il grande sommovimento tecnologico verso la virtual care, 
hanno spostato il centro di gravità della Medicina ${ }^{33}$ fuori dalllo spazio di incontro tra il medico e il paziente. La domanda se sia possibile oggi ritornare al centro e riporvi, sia pure in una luce nuova, il sodalizio medico-paziente è sensata. Molte buone ragioni fanno pensare che il rispristino è possibile, ma che si tratta di un compito faticoso, anche perché la consegna è in apparenza anti-storica e con ciò esposta al sospetto di tentazioni a ritorni impossibili. Si tratta invece di un dovere preciso che la Medicina Interna, in forza del suo olismo costitutivo, non può delegare. La Medicina Interna del futuro deve riprendersi uno spazio abbandonato, lasciato al caso e alla incompetenza.

Sorge però la domanda, scettica o supponente: in che modo? con che cosa farlo? La risposta è ingenua, disarmata: con gli strumenti storici del metodo classico, la anamnesi multidimensionale, l'esame obbiettivo e la loro sintesi, nella alleanza costitutiva etica, prima definita Medicina dell'ascolto. ${ }^{23,24}$ Certo, terzo passaggio, sarà arduo trovare il punto di saldatura tra la discrezione del metodo classico e il vigore della medicina scientifica (o tecnologica), nel momento in cui questa è tanto seducente e veleggia noncurante vincitrice. La dicotomia tra i destini (rimando alla rozza metafora delle quattro gambe del tavolo) di Medicina tecnologica e Medicina dell'ascolto ha prodotto uno scollamento che sembra insanabile. Eppure, per il bene del paziente, i due partners immaginari devono pur trovare una sintesi, sperimentarne il beneficio e sorvegliare perché esso non si disperda una seconda volta. Questa non è poi un auspicio solitario, velleitario. Il messaggio è ora propugnato con forza nella stessa direzione da Chang e Lee con il termine di Medicina interpersonale..$^{34} \mathrm{La}$ Medicina interpersonale è però - con qualche fastidio introduco una questione filologica - soltanto una variante semantica, letterale, del metodo classico. Rispetto ad esso la Medicina interpersonale immagina un legame ancora più stretto e vincolante tra medico e paziente in quanto lo pensa orientato e disciplinato verso un modello di cura che risponda alle situazioni, alle capacità e alle preferenze del paziente..$^{34}$ Questa ancora maggior attenzione al paziente rispetto al passato si spiega bene con la storia sociale dell'ultimo mezzo secolo: in esso il paziente ha guadagnato moltissimo in termini di riconoscimento di dignità civile. Ne è buon esempio la introduzione nella pratica della sperimentazione clinica controllata dei già citati PROs, cioè di un metodo standardizzato per misurare il punto di vista dei pazienti sul loro stato di salute. I PROs sono ora considerati parte centrale nella valutazione della qualità della vita e l'averli integrati alla raccolta routinaria ed al feedback dei pazienti ha molto migliorato il livello di cura. ${ }^{35-37}$ Così disegnata, la Medicina interpersonale, come la Medicina dell'ascolto, può essere vista come una attualizzazione laica, di storia civile, del modello classico.

Superare il senso del metodo classico nella sua versione ritrovata (o rinominata Medicina dell'ascolto o Medicina interpersonale) non significa ignorare il valore e la sostanza degli algoritmi diagnostici, prognostici e terapeutici. Solo, il metodo classico, nel farli propri, ridimensiona il concetto che essi possano autonomamente dominare il processo decisionale. Se le spettacolari conoscenze accumulate in questo fortunato mezzo secolo di Medicina hanno cambiato il nostro modo di vedere il malato e di offrigli ulteriori opportunità, esse non possono tuttavia mettere fuori causa il suo protagonismo umano, la sua spiritualità e la fecondità del suo rapporto con il medico: naturam expellas furca, tamen usque recurret ${ }^{38}$ cerchi di estromettere la naturalità del rapporto medico paziente con l'impeto della medicina tecnologica innovante ma esso ritorna, inesorabile. La riflessione generale, non banale tuttavia, è che non c'è scampo: in ogni anche lontanissimo futuro pensabile, oltre e dopo le acquisizioni che ci forniranno le nanotecnologie, la robotica, l'intelligenza artificiale, i $5 \mathrm{G}$ e database sempre più voraci e inappellabili, ci sarà sempre un paziente che cerca un medico per farsi visitare e chiedergli vicinanza e aiuto. ${ }^{39}$ Per questo la stessa dizione Medicina interpersonale in fondo, sembra in sé pleonastica: la medicina o è interpersonale o semplicemente non è.

\section{Un futuro per il metodo classico: le malattie a componente comportamentale dominante}

La rinascita del metodo classico potrebbe essere ancora più apertamente decretata, per altra via, dal prossimo - ma già incombente - futuro epidemiologico. Questo sarà dominato da un aumento di incidenza di malattie, già ampiamente diffuse, a forte componente comportamentale (Chang e Lee). Si pensi alla triade: diabete di tipo II, obesità, malattie cardiovascolari (per queste il riferimento è alla quota metabolica della eziologia). Alla loro base vi sono, fondamentali, i comportamenti personali, igienici, dietetici. Questi, di certo, non sono modificabili dalla medicina scientifica; lo sono invece, in grande misura, con il metodo diretto classico: esso li può rendere in larga parte emendabili se non addirittura sradicabili. Solo l'ascolto, il colloquio insistito e partecipe, la visita accurata trascinano insensibilmente il paziente a condividere la responsabilità delle scelte ed a modificare alla fine i suoi stili di vita. ${ }^{40,41} \mathrm{Il}$ metodo classico può fare anche di più nell'altra triade comportamentale funesta: droghe, alcol, suicidi. ${ }^{34}$ Qui il colloquio può significare addirittura salvezza. Si pone qui, in proposito, un tema cruciale sollevato da più parti ed è che, nella logica della divisione specialistica del lavoro, questi ambiti sono già 
presidiati da altre discipline, non mediche, organizzate. Non la penso così. Il mio pregiudizio è: senza la durezza di una laurea in Medicina e del suo tirocinio interminabile, il tipo di colloquio offerto da queste discipline è parziale, surrettizio, quando non solo di maniera.

Test di efficacia del metodo classico: impiego di biosimilari, switch di terapia, indagaine genetica

In modo un po' curioso, mi vengono in mente alcuni esempi di compiti recenti nel lavoro quotidiano del medico in cui è messa alla prova la tenuta del rapporto medico paziente. Essi potrebbero funzionare come test di valore del metodo. Il primo riguarda la capacità di convinzione - e di una futura buona compliance - che bisogna mettere in atto nel fare accettare al paziente l'impiego di farmaci biosimilari al posto degli originators cioè dei farmaci brand. Il secondo è lo switch tra un farmaco in trattamento storico, che ha assicurato al paziente benessere e sicurezza, e del quale si propone la interruzione per passare ad altro più fitting; viene in mente il passaggio dagli anti-vitamina $\mathrm{K}$ ai nuovi anticoagulanti orali, inibitori del Xa o della trombina (vale anche la eventualità contraria, il consiglio di continuare l'antivitamina $\mathrm{K}$ a fronte del desiderio del paziente di passare ad un farmaco più comodo). La difficoltà richiesta da entrambi questi passaggi non può essere messa solo in capo al medico visto come autorità tecnica che guida la partita e decide. Se prima non si è creata una tenuta solida tra i due destini - di paziente e di medico -, se non si è prodotto un tenace spirito di alleanza, questi passaggi diventano impraticabili. Questo, soprattutto quando, come nel caso dei biosimilari, si tratta di contemperare la libertà e la responsabilità del medico prescrittore, mosso dalla esigenza della salute del paziente, con la sostenibilità economica complessiva di un Servizio Sanitario in perpetua fragilità dei conti. Quando funziona, l'alleanza sortisce un altro effetto positivo (confesso qui la mia infatuazione civile!); essa non è soltanto il consiglio giusto dato dal medico al paziente ma è anche l'occasione e lo strumento per trasmettergli una sommessa lezione, di civismo. Senza alleanza non ci sono switch, senza alleanza dare un biosimilare sarà una sfida. Anche applicato ad una condizione sinora inedita, Il vecchio e povero rapporto medico-paziente mostra la sua versatilità nel tempo, segno impensato di modernità.

Una terzo esempio, meno incisivo forse, che misura la forza del rapporto sino a farne un ipotetico test di tenuta, è anche quello del paziente che si trova di fronte alla proposta di una indagine genetica. Se il paziente non viene preso per mano ed aiutato ad entrare in aspri territori scientifici, la proposta gli sembrerà sempre, anche se putativamente promettente, un avversario con cui misurarsi. Peraltro, in casi come questo la incertezza investe spesso, nella dimensione di cultura, lo stesso medico. Si mette allora in piedi una fragile ma onesta condivisione che aiuta a spogliare la nuova medicina della sua faccia più temibile.

\section{La saldatura di medicina tecnologica e medicina dell'ascolto. Per una medicina riformata}

Il medico che ha praticato il metodo clinico classico della tradizione italiana (e non solo) era, già lo si è detto, un medico scalzo, con pochi strumenti di sostegno ma fortissimo nella preparazione di dottrina e nella disposizione di persona. Il patrimonio positivo contenuto ed espresso nel suo tipo di pratica medica non si è svalutato nel tempo, vi sono dentro titoli che non risentono fluttuazioni.

L'avvento della medicina tecnologica e il suo dominio sembrano averne relegato sensibilità e comportamenti a fossili museali, da rispolverare, se mai, per un racconto del passato. Non è così. Se si ripensa al metodo clinico classico vengono anzi allo scoperto, come in controluce, i torti della medicina scientifica, primo tra essi l'aver se non mortificato, prudentemente evitato il suo connubio con il rapporto medico-paziente e con la sua storia fortunata. L'abbraccio acritico con tutto ciò che ci dà la tecnologia lascia un suo retrogusto che non è sempre gradevole. È vero che con la tecnologia non abbiamo perso il gusto buono della Medicina; soltanto, ne abbiamo un po' spento quello migliore.

La Medicina dell'ascolto (Portioli 1 e 2) o la più attualizzata Medicina interpersonale (Chang e Lee) pone qui, argomentandola, la sua esigenza di essere rimessa in piena luce, perché se ne possa cogliere con chiarezza il volto, per di più impreziosito da tutte le sfumature che la azione del tempo, con le sue esperienze e riflessioni, ha dato alla sua fisionomia. Riusare, con testa nuova, la ricchezza del paziente - la seconda inesplorata ricchezza, insieme alla nostra di curanti - e metterla a profitto non è un salto all' indietro, è semplicemente il dovere di non buttare nulla di quello che in questa dimensione è stato acquisito e che ritroviamo rilevante anche in tempi di virtual care. Per esempio, bruscamente, agli antipodi della/ma non in antitesi con la/medicina tecnologica, vi sono, nella nostra pratica clinica, temi estremi che non sono mai esplicitamente discussi. Concetti come compassione, paura, perdita sono spazi umani di confine, forse poco frequentati ma le cui tracce, con le loro penombre, sono ben rintracciabili nella trama del racconto del paziente. ${ }^{42}$ Farli emergere significa ritrovarsi con il senso proprio della Medicina che resta sempre quello di condividere fragilità. 
Ma dove avverrà la saldatura tra medicina interpersonale e medicina tecnologica? Nella intelligenza dei medici, intesa elementarmente come un sistema che è in grado di percepire un ambiente determinato e di agire al suo interno (Hawking). Nel contesto della medicina tecnologica sono premiati, della intelligenza, i versanti della razionalità, legati a nozioni di dottrina clinica, di congruenza matematica e statistica: ovvero la capacità, partendo da dati strumentali opulenti, di estrarre (le poche) inferenze corrette, prendere buone decisioni ed elaborare piani validi. Nella intelligenza, tuttavia, hanno posto anche altre figure mentali propriamente umane - la simpatia, la attenzione alla persona e al suo destino, l'immenso campo di gioco delle capacità istintive - che dalla razionalità non possono essere oscurate, e soccomberne. Sembra evidente l'interesse dei fedelissimi delle due bandiere di non contrapporsi e di non perdersi di vista. La saldatura è fertile, la sua strategia fa bene a tutti. Detto in modo apodittico: la Medicina non può essere solo scientifica ma non può essere, altrettanto, solo dell'ascolto (o interpersonale).

Da solo, riesumato, senza l'apporto della medicina scientifica o pretendendo di sostituirvisi in un ritorno ad una pratica medica verginale, il cosiddetto metodo classico, diventerebbe una sinistra efficiente fabbrica degli errori - mentre i nostri malati ci chiedono prima di tutto di non sbagliare.

Da sola, se si limita a proporsi come un intreccio ragionato - di macchine, algoritmi e statistica - cui delegare scelte e decisioni (e, in futuro vicino, anche opinioni) la medicina tecnologica corre il rischio di perdere per strada il paziente (Sackett et al.), di oscurarne soggettività e individualità. La loro saldatura genera invece una ricchezza diagnostica inedita.

Questa saldatura è anche urgente. Sulla pratica medica pesa l'incognita di una deriva minacciosa. La cosiddetta sanità digitale pone addirittura a suo fondamento una medicina a distanza dal malato. I suoi strumenti in sé tecnicamente preziosi - controllo dei parametri vitali tramite smart watch, braccialetti, sistemi di alert - ci paracadutano in un universo stellare in cui poi, presto, arriverà il conteso $5 \mathrm{G}$, la rete ultraveloce che, moltiplicando all'infinito la capacità di trasmissione di dati, promette di curare il paziente sempre più da remoto. Con orgoglio le app annunciano di poter visitare il paziente in $3 \mathrm{D}$ come se fosse davanti al medico. Non è finita. È già presentata come frontiera (quante frontiere!) della medicina la creazione, per ognuno di noi di un digital twin, di un gemello digitale, che sia una copia esatta di ogni paziente, ${ }^{43}$ inteso, il gemello, non solo come un archivio di ogni suo dato clinico ma come una replica virtuale completa di tutto il suo corpo. Su questo corpo digitale, sul nostro avatar, sembra possibile eseguire ogni tipo di test come, per esempio, verificare gli effetti collaterali che un farmaco può avere su di lui e solo su di lui (cioè su di noi). L' impersonale è arrivata ad una radicalità grottesca. Il problema è che nella stessa direzione cammina, senza esitazione, il paziente aggiornato. Diseducato al rapporto con il suo medico, egli pensa che sia ormai possibile abdicarvi. Da tempo egli è entrato, perché vi si riconosce, in questa ottica che non gli appare affatto diminutiva o rinunciataria: per i suoi problemi di salute il $17 \%$ dei cittadini usa WhatsApp, il 15\% sms, il 19\% le e-mail.

Medicina tecnologica e Medicina dell'ascolto - o Medicina interpersonale - sono dunque urgentemente chiamate ad avere un solo 'metodo', indivisibile. Si tratta di uno stato di necessità. Con questo passo rivoluzionario, semplicemente, si metabolizza il passato, riproponendolo in una sintesi positiva con un presente che è dominante ma che non può escluderlo dal gioco. Dall'incontro le due parti escono rafforzate in pari misura.

Dentro un 'nuovo' rapporto medico-paziente questo sì è visionario - si potrebbe addirittura esprimere un potenziale educativo non solo sui due contraenti, paziente e medico, ma sullo stesso più largo contesto umano in cui entrambi vivono e lavorano. Da un evento privato, cellulare, molecolare, potrebbe partire un richiamo ad un più generale rispetto alla dignità dell'uomo, in qualunque altra platea sociale essa venga messa alla prova (quando non sottomessa o umiliata).

La saldatura (fusione? sintesi?) tra medicina tecnologica e medicina dell'ascolto costituisce, come usa dire in logica, un salto di paradigma. Vince un altro olismo, ben riuscito, tra scienza e pratica.

Il lavoro da fare è molto, e arduo. Il primo compito sarà togliere gli strumenti della medicina interpersonale - l'ascolto, la attenzione, la condivisione - usurpati, dalla presa salda di faccendieri dilettanti. Una volta depurato e riportato alla vecchia dignità, il metodo classico potrà essere ridato in dote alla medicina scientifica che lo aveva accantonato, nella presunzione non manifesta di poter fare da sola. Il metodo classico attraverso la sua investitura di antica storia umana porterebbe la tecnologia stessa a trascendere la sua sola dimensione materiale. A quel punto, la Medicina riavrebbe il suo volto grandioso.

La pedagogia di questa riflessione è semplice. Prima di rifiutare il passato bisogna saper ben discernere. Non è il caso di dire con Verdi torniamo all'antico e sarà un progresso ${ }^{44}$ ma il Vescovo Ambrogio indica una guida sicura sul compito di chi ricerca: ut et nova quaerat et parta custodiat. ${ }^{45} \mathrm{Si}$ devono cercare, quasi compulsivamente, cose nuove ma continuando a custodire le buone tra quelle partorite dal tempo. 


\section{Conclusioni}

Il metodo clinico classico della scuola medica italiana non era un corpus dottrinale insegnato e consegnato stabile ma un processo in movimento. In questa sua natura dinamica era condizionato dagli strumenti diagnostici prevalenti e quasi esclusivi del tempo: la anamnesi e l'esame obbiettivo. La teorizzazione dei suoi principi e fondamenti è stata una operazione a posteriori, generata dal ripensamento della sua attuazione concreta nel tempo. La sua derivazione dal modello compatto centro-europeo, con malattie chiuse (e di proprietà), gli ha consentito di passare da una capacità di valutazione intuitiva ad una di tipo qualitativo; nell'incontro, induzione empirica e deduzione di dottrina si sono mescolate e sommate in un gioco di rimandi, complementarità e reciprocità. Saldezza dei riferimenti di cultura e forza del rapporto medicopaziente hanno prodotto un risultato olistico pieno, nel senso di generare attenzione e competenza sull'intero soma del malato e comprensivamente, in modo armonioso, del suo mondo psichico. Questo ha garantito nel tempo al metodo classico autonomia culturale rispetto a più recenti discipline parcellari, frazionanti e tentativamente vicarie. La assenza di vincoli temporali nella azione diagnostica gli ha anche consentito, nei casi più fortunati, di tentare la costruzione, per una didattica nuova, delle storie naturali di alcune malattie. La Medicina dell'ascolto e/o Medicina interpersonale oggi reclamata è solo una attualizzazione dei contenuti positivi del metodo classico, Il suo riproporsi in una veste rinnovata. $\mathrm{La}$ saldatura della potenza della medicina tecnologica con il patrimonio di umanità del metodo clinico classico può ridare alla Medicina il suo volto grandioso.

\section{Post scriptum}

Lo spirito di queste riflessioni sul metodo classico della tradizione medica italiana è spirito irregolare, la sua rievocazione è indisciplinata. Essa evita istintivamente gli approcci dogmatici, di sistema, e sceglie una più dimessa cronaca del modo di stare con il paziente. In altre parole, non ha la disposizione di esplorare e definire una qualità teorica del metodo ma quella di catturarne, dalla profondità della esperienza, la maggior quantità possibile di reale, umano e tecnico. Forse l'approccio descrittivo - se non (talora) colpevolmente letterario - della riflessione maschera il suo intento vero che è invece di testimoniare con forza la tensione verso una Medicina Riformata. La Medicina Interna italiana è pronta per affrontare e determinarvi un futuro intrinsecamente originale, suo proprio, non delegabile.

\section{Bibliografia}

1. Ferraris M. Pensiero in movimento. Milano: Paravia Editore; 2019.

2. Augias C, Filoramo G. Il grande romanzo dei Vangeli. Torino: Einaudi Editore; 2019.

3. Federspil G, Scandellari C. Medicina in: Gli strumenti del Sapere Contemporaneo - Vol I: Le Discipline. Torino: UTET; 1985.

4. Federspil G, Giaretta P, Rugarli C, et al. Filosofia della Medicina. Milano: Raffaello Cortina Editore; 2008.

5. Garimberti U. Freud e lo spostamento dello sguardo. in Sublimazione: Dizionario di Psicologia. Torino: UTET; 1992.

6. Murri A. Pensieri e precetti (a cura di Antonio Gnudi e Alberto Vedrani). Bologna: Zanichelli Edit., 1924.

7. Vico GB. Scienza nuova (verum ipsum factum). $3^{\text {a }}$ edizione, 1744, libro I, sez. 3.

8. Ferraris M. Goodbye Kant. Milano: Bompiani Editore, 2004.

9. Sotgiu G, Murri A. Un maestro di medicina e di inseganamento conferenza 1972, Bologna, Palazzo Montanari (in: La nascita del concettto di clinica negli scritti medici e in altre opere di Augusto Murri) 70, 2016, pp. 3200-3207.

10. Gnerre P, Nardi R. Metodi di ragionamento clinico e strumenti utilizzati nella pratica clinica in ambito internistico ai fini decisionali. Quad Ital J Med 2020; 8(1):19-30.

11. Cartesio (Renè Descartes). Meditazioni metafisiche (1641) in: M. Ferraris Cartesio e la nascita della filosofia moderna, p. 88, da Renato Cartesio, Opere, a cura di Eugenio Garin. Bari: Laterza; 1967.

12. Baricco A. The Game. Torino: Einaudi editore; 2018.

13. Dante. La Divina Commedia, Par XXVIII, 91-93.

14. Malaguzzi L. Esperienze per una nuova scuola dell'infanzia. Atti Seminario di Studio, Reggio Emilia, 1971.

15. Malaguzzi L. I cento linguaggi dei bambini. Edizioni Junior; 1975.

16. Scandellari C. Augusto Murri e il suo pensiero metodologico. Bollettino della facoltà di Medicina e Chirurgia dell'Univeresità di Ancona, 2000; III (7-8): 12.

17. Salvatore G. Comunicazione personale, 1983.

18. Maccacaro GA. The median in measurements in experimentation. Arch Sci Biol (Bologna) 1955;39:156.

19. Maccacaro GA, et al. Biometria. Principi e metodi per studenti e ricercatori. Padova: Piccin Editore; 1978.

20. Gilbert A, Sebag-Montefiore D, Davidson S, Velikova G. Use of patient-reported outcomes to measure symptoms and health related quality of life in the clinic Gynecol Oncol 2015;136:429-39.

21. Papi G. Il censimento dei radical-chic. Milano: Feltrinelli Ed.; 2019.

22. Donelli D, Antonelli M, Rizzato M, Morini L. Empathic disconnection and the patient-physician relationship in clinical practice. Ital J Med 2018;12:265.

23. Portioli I. Medicina tecnologica e medicina dell'ascolto. Rel. In: 'Il paziente fragile', Teatro Bibiena, Mantova, 4 marzo 2006 (Az. Osp. 'C. Poma' di Mantova).

24. Portioli I. Fragilità del malato e valore etico dell'ascolto. 
Intervista a 'La Cittadella', 9 giugno 2006, pag 16-17.

25. Verghese A. Culture shock: Paient as Icon, Icon as Patient. N Engl J Med 2008;359:2748.

26. Wenzel RP. Medicine, Technology, and Physician Loneliness. N Engl J Med 2019;380:305.

27. Kulkarni A. Navigating Loneliness in the era of virtual care. N Engl J Med 2019;380:307.

28. Smith R. Why are doctors so unhappy? BMJ 2001;322:1073.

29. Steinehjem K, Herren D, Pulver G, et al. Association of frequency of lipid 1 testing with changes in lipid lowering therapy JAMA Intern Med 2017;177:1579.

30. Davies L, Hendrickson CD, Hanson GS. Experience of US patients who itself-identify as having an overdiagnosed thyroid cancer: a qualitative analysis. JAMA Otorinolaryngol Head Neck Surg 2017;143:663.

31. Sinsky C, Culligan L, Li L, et al. Allocation of physician time in ambulatory practice; a time and motion study in 4 specialties. Ann Intern Med 2016;165:753.

32. Sackett DL, Rosenberg WM, Gray JA, et al. Evidencebased medicine: what it is and what it isn't. BMJ 1996;312:71.

33. Lee TH. Eulogy for a quality measure. N Engl J Med 2007;357:1175.

34. Chang S, Lee TH. Beyond evidence-based medicine. N Engl J Med 2018;379:1983.

35. Perlis N, Krahn MD, Boehmen KE, et al. The bladder utility symptom scale (BUSS): a novel patient-reported outcome instrument in bladder cancer. J Urol 2018;piiS0022-5347(18)42498-6.

36. Calvert M, Kyle D, Metrcieca-Bebber $P$, et al.
Guidelines for inclusion of Patient-reported outcomes in clinical trials protocols. The SPIRIT-PRO extension. JAMA 2018;319:483-94.

37. Van Der Wees PJ, Nijhuis-Van der Sanden MW, Avanian $\mathrm{JZ}$, et al. Integrating the use of patient-reported outcomes for both clinical practice and performances measurement: views of experts from 3 countries. Milbank Q 2014;92:754-75.

38. Orazio. Epist I, 10, 24.

39. Haas JS, Cook EF, Puopolo AL, et al. Is the professional satisfaction of general internists associated with patient satisfaction? J Gen Intern Med 2000;15:122.

40. Zolnierek KB, Dimatteo MR. Physician communication and patient adherence to treatment: a metanalysis. Med Care 2009;47:826.

41. Kelley JM, Kraft-Todd Gschapira L, Kossowky J, Russel H. The influence of the patient-clinician relationship on healthcare outcomes: a systematic review and meta-analysis of randomized clinical trials PLoS One 2014;9:e94207.

42. Sims T, Tsai JL. Patients respond more positively to physician who focus on their ideal affect. Emotion 2015;15:303.

43. Cheran G. Il gemello sanitario digitale, Intervista di L. Grassia, La Stampa 10 sett. 2019.

44. Verdi G, citazioni verdiane (non pubblicate).

45. Ambrogio di Milano De Paradiso, 25 in: Il Paradiso - Il giardino piantato a Oriente. Città del Vaticano: Paoline Edizioni; 2018. 


\title{
Modelli di ragionamento clinico e strumenti utilizzati nella pratica clinica in ambito internistico ai fini decisionali
}

\author{
Paola Gnerre, ${ }^{1}$ Roberto Nardi ${ }^{2}$ \\ ${ }^{1}$ Medicina Interna 2, Ospedale San Paolo, Savona; ${ }^{2}$ Medicina Interna, Bologna, Italia
}

\section{Introduzione}

La diagnosi clinica è il processo d'identificazione o di determinazione della natura e della causa di una malattia attraverso la valutazione della storia del paziente, dell'esame obiettivo e della revisione dei dati disponibili. ${ }^{1}$ Il ragionamento clinico è il processo attraverso il quale i medici raccolgono, elaborano ed interpretano le informazioni acquisite sui pazienti, per sviluppare un piano diagnostico-terapeutico ed assistenziale, a partire dai dati anamnestici, attraverso l'esame fisico, l'osservazione seriale dell'ammalato e i risultati degli accertamenti proposti. Il medico genera una serie di diagnosi differenziali fino a definire un orientamento diagnostico conclusivo. Questi passaggi sono effettuati, di norma, in maniera interattiva e dinamica, tanto da dover modificare, in aluni casi, la diagnosi iniziale sulla base dell'interazione con il paziente e delle nuove informazioni che nel tempo sopraggiungono. Durante il processo diagnostico il medico internista deve confrontarsi sostanzialmente con diverse sfide: i) informazioni superficiali ed ambigue, a volte 'ritenute': i dati sono spesso acquisiti in maniera parziale e frammentata nel tempo o devono essere indirettamente assunti dai familiari o dal caregiver. In alcuni casi il paziente stesso tende a sottovalutare o a non dichiarare alcuni sintomi, anche per paura di un iter diagnostico problematico, della conseguente diagnosi e della stessa ospedalizzazione;

Corrispondente: Paola Gnerre, Ospedale San Paolo Savona, Italia.

E-mail: pgnerre@yahoo.it

Ringraziamenti: Si ringrazia il dott. Maurizio Ongari, Direttore di Medicina Interna dell'Ospedale di Porretta (AUSL di Bologna) per la revisione del caso presentato.

Articolo pubblicato secondo la Creative Commons Attribution NonCommercial 4.0 License (CC BY-NC 4.0).

${ }^{\circ}$ Copyright: the Author(s), 2020

Licensee PAGEPress, Italy

QUADERNI - Italian Journal of Medicine 2020; 8(1):19-30 ii) aspecficità dei sintomi: alcuni sintomi segnalati e/o osservati, di tipo costituzionale possono essere talmente aspecifici, da poter indicare diagnosi molteplici, tanto da doverli considerare come potenziali falsi amici del percorso diagnostico; iii) patologie sottese: in alcuni casi esistono co-morbilità multiple e patologie 'ad iceberg' sottese, ma oligoasintomatiche, in grado di modificare il decorso clinico-prognostico della malattia principale e l'orientamento decisionale del medico per la valutazione del problema saliente; iv) condizioni mutevoli e di incertezza: lo stato di un paziente può cambiare in qualsiasi momento nel corso del processo diagnostico: il medico ha bisogno di un tempo adeguato per rivedere correttamente la diagnosi (la cosiddetta epicrisi); v) complessità: il paziente può essere talmente complesso da non essere supportato, per le sue multi-patologie, da indicazioni utili dalle linee guida disponibili. In questi casi il medico esperto e consapevole anche delle opinioni del paziente può decidere in piena autonomia, senza contrastare le regole della Evidence Based Medicine; ${ }^{2}$ vi) appropriatezza e possibili errori nelle informazioni acquisite: il medico richiede accertamenti e indagini strumentali allo scopo di ottenere ulteriori dati per confermare o smentire un'ipotesi diagnostica, ma, in alcuni casi - soprattutto se l'ipotesi diagnostica è incerta o ambigua - tali richieste, se inappropriate, inducono ulteriori errori gestionali del paziente. ${ }^{3}$

\section{L'importanza dell'anamnesi e dell'esame obiettivo per un corretto metodo clinico: considerare l'ipotesi diagnostica iniziale per le decisioni successive}

L'anamnesi è la raccolta diretta da parte del paziente e/o dei suoi familiari di tutte le informazioni necessarie al medico per orientare la diagnosi rispetto al problema clinico rappresentato. Mentre il clinico ascolta l'ammalato, inizia a sviluppare un'impressione della storia del paziente, secondo un'astrazione mentale, che guiderà ulteriori quesiti ed approfondimenti, fino alla rappresentazione del 
problema, che incorpori le caratteristiche più importanti del caso, passo essenziale per sintetizzare i dati e formulare un piano di diagnosi e trattamento. ${ }^{4}$ Nonostante i continui progressi tecnologici e con tutti limiti evidenziati, l'anamnesi e l'esame obiettivo del paziente rimangono una componente fondamentale della valutazione clinica, ai fini dell'orientamento diagnostico: la prima ipotesi diagnostica si basa in gran parte sui dati acquisiti in questo iniziale approccio con il paziente. L'insegnamento dell'esame obiettivo direttamente al letto del malato è da sempre ritenuto di grande importanza. Esso consente l'acquisizione di molte informazioni cliniche, mediante metodi, procedure e manovre utili per riconoscere i principali segni patologici di determinate condizioni e/o malattie. L'esame físico viene insegnato tradizionalmente come una lista (peraltro riportata nel format delle nostre cartelle cliniche) di caratteristiche che devono essere descritte in ogni paziente per ogni organo e/o apparato. L'esame obiettivo guidato dall'ipotesi, invece, insegna a sviluppare le abilità nel ragionamento diagnostico. ${ }^{5} \mathrm{~A}$ differenza del tradizionale esame, effettuato in senso cranio-caudale (con il classico metodo: ispezione, palpazione, percussione, auscultazione e manovre particolari aggiuntive della semeiotica tradizionale) l'esame fisico guidato dall'ipotesi, in base ai problemi attivi, utilizza il sintomo/disturbo specifico del paziente per orientare l'iniziale ipotesi diagnosticodifferenziale e procedere alla ricerca di specifici segni compatibili con tale sospetto. ${ }^{6}$ Saper effettuare correttamente un esame obiettivo aiuta il ragionamento clinico, supporta l'appropriatezza delle indagini da richiedere e favorisce un approccio consapevole alla diagnosi, attraverso la ricerca di specifici elementi, allo scopo di confermare o escludere l'ipotesi diagnostica iniziale. ${ }^{7}$ Purtroppo l'insegnamento sul come effettuare un esame obiettivo al letto del paziente è una pratica che si è progressivamente ridotta, fin dagli anni ' $70,{ }^{8}$ con il rischio conseguente di una crescita a dismisura di richieste di indagini ed accertamenti diagnostici inappropriati. ${ }^{3}$

\section{Le diverse tipologie delle modalità di ragionamento clinico}

Le diverse tipologie delle modalità di ragionamento clinico che stanno alla base delle decisioni mediche, con le relative caratteristiche differenziali, sono schematizzate nella Tabella 1. Le due forme di ragionamento presentate non si escludono vicendevolmente e possono essere interattive e non necessariamente sequenziali: esse contribuiscono in modo complementare all'accuratezza complessiva del processo decisionale. In generale coloro che impiegano entrambi i modelli ottengono migliori risultati., ${ }^{910}$

\section{Il metodo induttivo-euristico}

Il modello intuitivo-euristico è un metodo approssimativo, intuitivo, analogico, non analitico, semplificativo, con un approccio quasi automatico e passivo alla soluzione dei problemi. Esso si basa su poche sintetiche informazioni, acquisite a volte anche in maniera sensoriale, con il solo colpo d'occhio,

Tabella 1. Caratteristiche dei diversi modelli alla base delle decisioni mediche: intuitivo-euristico e analitico-ipoteticodeduttivo.

\begin{tabular}{|c|c|c|}
\hline & MODELLO INTUITIVO-EURISTICO & MODELLO ANALITICO, IPOTETICO DEDUTTIVO \\
\hline $\begin{array}{l}\text { Elaborazione cognitiva } \\
\text { a carattere... }\end{array}$ & $\begin{array}{l}\text { Approssimativo, intuitivo, analogico, } \\
\text { non analitico, semplificativo }\end{array}$ & $\begin{array}{l}\text { Sistematico, basato sull'ipotesi e sul ragionamento } \\
\text { clinico deduttivo (investigativo) }\end{array}$ \\
\hline Basato su... & $\begin{array}{c}\text { Induzione per schemi mentali } \\
\text { e per pattern recognition porta } \\
\text { generalmente ad una singola ipotesi diagnostica }\end{array}$ & $\begin{array}{l}\text { Valutazione di una serie di ipotesi e delle } \\
\text { probabilità in gioco, utilizza metodi Bayesiani }\end{array}$ \\
\hline Dettagli richiesti & $\begin{array}{l}\text { Scarsi, poche sintetiche informazioni, acquisibili } \\
\text { in alcuni casi anche con il solo colpo d'occhio }\end{array}$ & $\begin{array}{c}\text { Richiede numerose informazioni per ottimizzare } \\
\text { il risultato }\end{array}$ \\
\hline Velocità & Rapida, impiegato dai più esperti & Lenta, più spesso utilizzato da medici giovani \\
\hline Evidence based & No, l'esperienza migliora l'efficienza & Si, con i limiti posti dal mondo reale e dai pazienti complessi \\
\hline Potere predittivo & Basso & Elevato \\
\hline Rigore scientifico & Scarso & Alto \\
\hline Limiti & L'eccessiva sicurezza può portare ad errori & Eventuali bias di fondo possono portare a risultati distorti \\
\hline Rischi (vedi testo) & $\begin{array}{l}\text { Errore di euristica inadeguata: la situazione è } \\
\text { troppo complessa per poter essere schematizzata } \\
\text { o semplificata }\end{array}$ & $\begin{array}{l}\text { Errore di comprensione: le premesse o le conclusioni } \\
\text { sono sbagliate }\end{array}$ \\
\hline
\end{tabular}

Modificata da Rosselli, 2007..$^{10}$ 
affidato in alcuni casi solamente all'intuito personale, quasi viscerale, di pancia o d'istinto ('gut feeling' in lingua inglese) e/o alle circostanze estemporanee. Tale approccio presenta evidentemente diversi limiti: ${ }^{11}$ i) euristica di rappresentatività: si valuta una possibile diagnosi senza considerare la reale probabilità di malattia, ma solamente in base ad impressioni personali correlate ad esperienze precedenti o a decisioni emotive, condizionate da un contesto stringente (il cosiddetto 'framing bias', in relazione alle richieste dei pazienti, dei famigliari, dell'azienda sanitaria o ad altre motivazioni estrinseche); ii) euristica di disponibilità: si valuta una possibile diagnosi sulla base del richiamo di casi, a volte assai rari, occorsi di recente o appena acquisiti dalla lettura di una rivista scientifica; iii) euristica di ancoraggio: si valuta una possibile diagnosi persistendo nell'ipotesi diagnostica teorica iniziale, nonostante l'acquisizione di nuovi dati che potrebbero giustificare alternative migliori; iv) euristica di presunzione (ovvero di 'over confidence'): eccessiva sicurezza nelle decisioni intraprese; v) euristica inadeguata: la situazione è troppo complessa per poter essere schematizzata o semplificata.

\section{Il metodo ipotetico-deduttivo}

Il metodo ipotetico-deduttivo è il modello di decisione clinica maggiormente utilizzato in Medicina Interna. Osservare, valutare, concatenare e dedurre. Sono questi i principi che, attraverso la magistrale penna di Sir Arthur Conan Doyle, Sherlock Holmes rivela alla sua spalla Watson. Questa strategia viene, con altre modalità, impiegata dai medici per casi particolarmente complessi o in cui siano presenti elementi di incertezza. È infatti molto simile a quella praticata da un esperto detective che, con specifiche abilità di osservazione, deduzione, conoscenza e vasta esperienza su casi reali, nonché di valutazione del contesto psico-ambientale e delle potenziali incongruenze tra dati disponibili e conclusioni immediatamente emergenti, raccoglie indizi su un particolare caso. ${ }^{12} \mathrm{Il}$ medico effettua una serie di ipotesi sulla possibile diagnosi del paziente attraverso l'analisi dei sintomi presentati dal paziente, la storia clinica e i reperti obiettivi. In questo approccio possono essere comprese strategie quali l'esclusione limitata di diagnosi possibili ('restricted rule-out') e la definizione graduale della diagnosi ('stepwise refinement'). Attraverso questo processo, viene identificato un programma diagnostico, atto a stabilire la diagnosi e la successiva azione terapeutica. L'ipotesi diagnostica viene successivamente perfezionata con ulteriori dati clinici e il medico conferma o smentisce la sua ipotesi sulla base del decorso clinico e dei risultati dei vari test diagnostici. Durante tale verifica il clinico valuta: i) l'adeguatezza dell'ipotesi: disponiamo di dati sufficienti per formulare un'ipotesi verosimili? La presentazione clinica è coerente con l'ipotesi iniziale?; ii) l'appropriatezza: sono appropriati i collegamenti causali o fisiopatologici? Nella nostra valutazione abbiamo distorto la comprensione dei fatti e delle evidenze disponibili?; iii) la coerenza: l'ipotesi diagnostica offre la più congruente spiegazione con la sintomatologia presentata dal paziente?; iv) la comprensione: le premesse o le conclusioni sono sbagliate? Una difettosa generazione di ipotesi diagnostiche e una chiusura prematura del caso clinico sono le principali carenze di questo metodo. Un suo vantaggio invece è la flessibilità adattativa, in funzione delle epicrisi che si rendono di volta in volta necessarie, a seconda della complessità del caso, che consente di correggere il tiro ed evitare possibili errori (Figura 1). ${ }^{8}$

\section{Ragionamento clinico e processo decisionale: le sole conoscenze non bastano}

Il percorso che induce il medico ad ipotizzare una diagnosi, a partire da una serie di informazioni, è uno dei compiti più difficili. Il ragionamento clinico descrive in sostanza i processi mentali complessi che vengono coinvolti nell'elaborazione della decisione finale. Esso si attua attraverso due fasi sostanziali. i) La prima è costituita dalla valutazione attenta e dalla raccolta/selezione dei dati salienti: richiede specifiche e selettive capacità di osservazione (ovvero l'acquisizione delle informazioni primarie), nonché di tipo relazionale, psico-emotivo ed empatico. ii) La seconda è quella della decisione clinica, sulla base dei dati acquisiti: richiede conoscenza, competenza, intuito, visione (ovvero guardare oltre $i$ dati, al fine di 'metterli insieme'), anche in maniera creativa. Deve essere mantenuto un elevato livello di vigilanza - anche nei confronti di possibili trappole, unitamente ad un'irrinunciabile capacità di giudizio e di buon senso.

Consideriamo, ad esempio, un caso clinico come quello rappresentato in Tabella 2.

Molti medici lamentano diverse carenze nell'insegnamento sulla metodologia clinica. Si continua a proporre una metodologia tradizionale, basata - pur se con argomentazioni assolutamente legittime - su anamnesi ed esami obiettivi standard. Le modalità di valutazione della miriade di segni e sintomi proposti dalla semeiotica classica, in assenza di una ragionamento complessivo su cui far scaturire le ipotesi diagnostiche non è molto utile, anzi, a volte, si limita ad una pedissequa, formale, acritica ed asettica compilazione di dati in cartella clinica, senza un esplicito orientamento diagnostico. La selezione dei bisogni attivi del paziente è invece fondamentale per una loro corretta gestione. Le abilità cognitive stanno alla base della diagnosi e di ogni azione 
Tabella 2. Caso clinico.

Un uomo di 58 anni con una lunga storia di ipertensione arteriosa, pregressi episodi di dispnea da sforzo e sporadiche lipotimie, si presenta al Pronto Soccorso per palpitazioni, dispnea con ortopnea e riferito precedente rilievo di soffio cardiaco. Un primo esame obiettivo evidenzia turgore delle giugulari e soffio in crescendo-decrescendo sul focolaio di Erb, irradiato al margine sternale superiore e a sinistra verso l'ascella.

Dopo la somministrazione di furosemide per via endovenosa e nitrati sublinguali, il paziente presenta un improvviso deterioramento clinico, con grave ipotensione e segni di shock cardiogeno. La somministrazione di dopamina per via endovenosa e la successiva somministrazione di noradrenalina non migliorano il quadro clinico.

Un secondo medico, intervenuto di seguito, nota un impulso carotideo bisferiens. Associando questo rilievo semeiotico alla risposta apparentemente paradossa alla terapia farmacologica (peggioramento con riduzione del pre-carico e mancata risposta agli inotropi) ipotizza una possibile cardiomiopatia ipertrofica ostruttiva da ipertrofia asimmetrica del setto.

\section{Commento}

La situazione descritta deriva da un restringimento del tratto di efflusso del ventricolo sinistro (VS), con gradiente pressorio sistolico sottovalvolare aortico, ipercinesia ventricolare, aumentata velocità di flusso, apposizione del lembo anteriore della mitrale al setto interventricolare (effetto Venturi) e conseguente doppio impulso carotideo.

In questo caso il medico subentrato in seguito mostra, rispetto agli altri colleghi, una strategia clinica più efficace, dimostrando un'encomiabile conoscenza delle malattie cardiovascolari e delle varie cause di dispnea, lipotimia, sincope e shock cardiogeno e di saper adattare questa sua competenza, guardando oltre il contesto della situazione clinica. Ha infatti ipotizzato che la dispnea del paziente, associata al polso carotideo bifasico e ad una risposta inadeguata alla terapia farmacologica fosse conseguenza di un'ostruzione della camera di efflusso del VS con disfunzione diastolica ed ha incluso l'ipertrofia del setto asimmetrico come una delle probabile cause.

Questo esempio ci mostra come gli errori decisionali vanno probabilmente oltre le conoscenze acquisite e/o carenti (ovvero le abilità cognitive), ma coinvolgono la capacità di un ragionamento clinico epicritico e il conseguente processo decisionale.

Modificato da Harpartap e Carpenter, 2006. ${ }^{13}$

\section{Le fasi del processo del ragionamento clinico (NEL MODELLO ANALITICO-IPOTETICO DEDUTTIVO)}

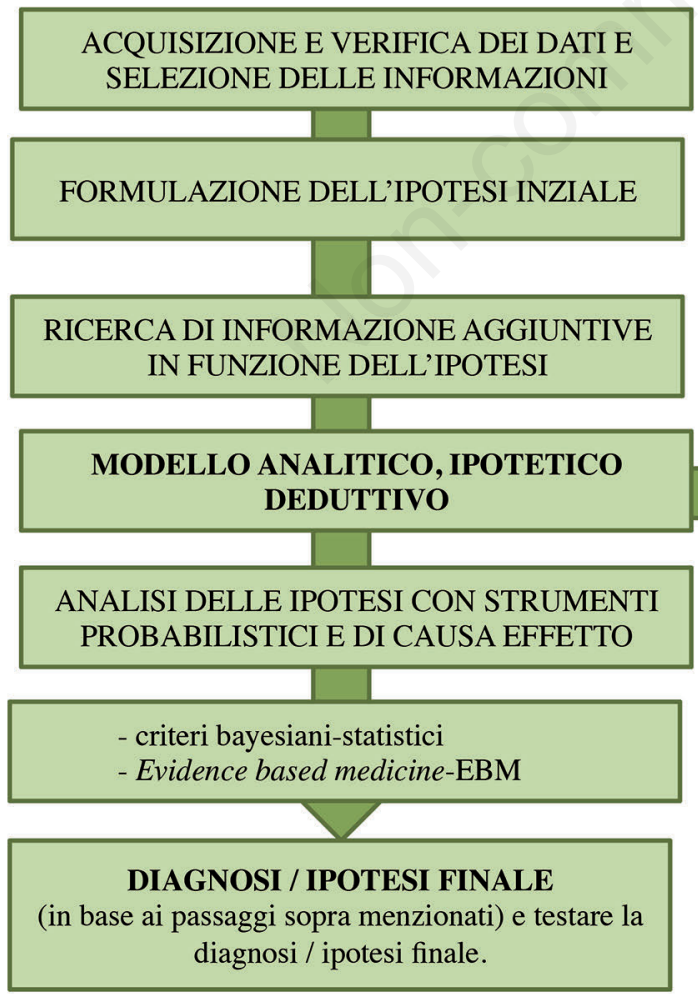

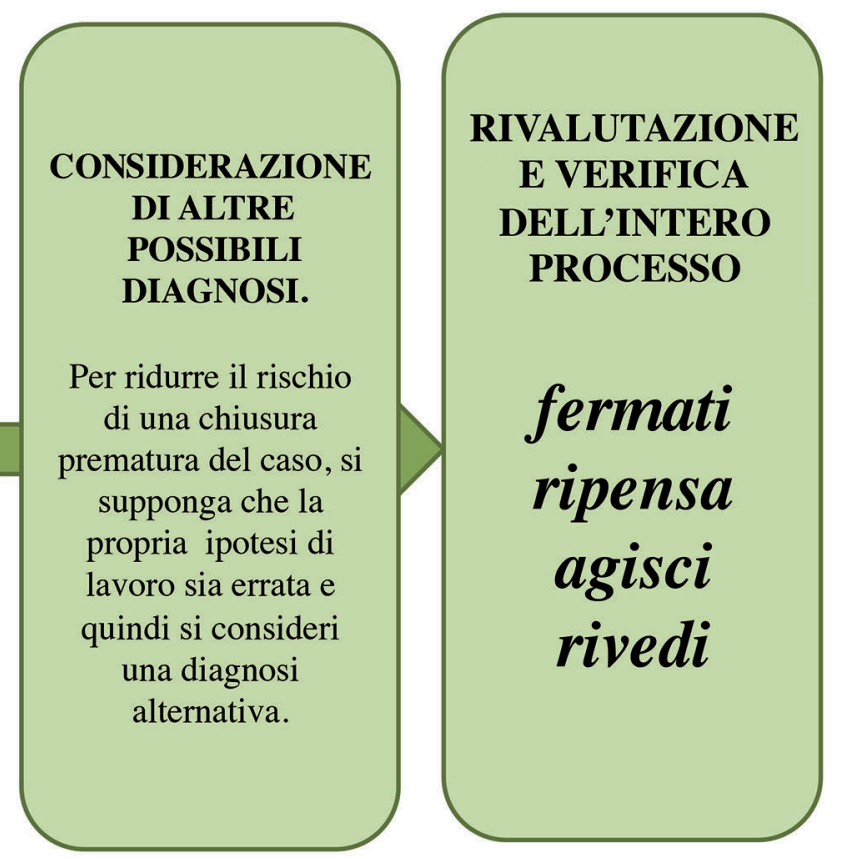

Modificata da Carver College of Medicine, University of OHIO, Dept of Internal Medicine, Clinical and Diagnostic Reasoning

Figura 1. Metodo ipotetico-deduttivo: fasi del ragionamento clinico. 
terapeutica, ma l'eccellenza medica si fonda su altre essenziali competenze di livello gerarchico superiore, che vanno oltre la mera conoscenza. ${ }^{14}$ L'internista dovrebbe essere in grado di richiamare sul singolo caso non solamente le sue cognizioni, ma anche le sue capacità di comprendere il contesto e di selezionare in maniera responsabile le informazioni prioritarie, agendo di conseguenza con decisioni appropriate.

\section{Strumenti di ragionamento clinico}

Diverse strategie sono utilizzate nel processo decisionale medico, con strumenti spesso implicitamente utilizzati, anche inconsapevolmente, nella pratica clinica (Tabella 3 ).

A seconda dell'ambito di cura verrà prevalentemente utilizzato un modello di ragionamento rispetto ad un altro. Ad esempio, in medicina d'urgenza il medico non si concentra solamente sulla diagnosi probabile, ma deve soprattutto pensare ad escludere condizioni potenzialmente letali o altre problematiche che potrebbero mettere in pericolo il paziente (Tabella 4).

I processi decisionali possono basarsi su pensieri veloci (rapidi, automatici, spesso efficaci ma rigidi) o su pensieri lenti (analitici, controllati, faticosi ma flessibili ed adattativi). Nella medicina moderna, spesso gravata da impellenze organizzative, il modello veloce si sta sempre più affermando, anche se contrassegnato da maggior rischio di errore. ${ }^{15}$ Le strategie lente vengono utilizzate come strumento di approfondimento della diagnosi rapida o a fronte di problemi complessi, non immediatamente inquadrabili

Tabella 3. Strategie di decisioni cliniche: caratteristiche salienti e possibili limiti.

\begin{tabular}{|c|c|c|c|c|c|}
\hline Pattern recognition & $\begin{array}{l}\text { Linee guida, flow } \\
\text { charts e algoritmi } \\
\text { (modello } \\
\text { algoritmico- } \\
\text { cook-book) }\end{array}$ & $\begin{array}{l}\text { Metodo ipotetico- } \\
\text { deduttivo }\end{array}$ & $\begin{array}{l}\text { Event driven } \\
\text { (guidato da eventi) }\end{array}$ & $\begin{array}{l}\text { Rule out worst-case } \\
\text { scenario }\end{array}$ & $\begin{array}{l}\text { Metodo exhaustion } \\
\text { (metodo esaustivo) }\end{array}$ \\
\hline $\begin{array}{l}\text { Richiede la } \\
\text { interiorizzazione e la } \\
\text { memoria di una serie di } \\
\text { esperienze cliniche } \\
\text { precedenti }\end{array}$ & $\begin{array}{l}\text { Livello più } \\
\text { semplificato } \\
\text { (in funzione di dati } \\
\text { pre-definiti) nella } \\
\text { gerarchia } \\
\text { decisionale clinica }\end{array}$ & $\begin{array}{l}\text { Modello di decisione } \\
\text { clinica } \\
\text { maggiormente } \\
\text { utilizzato }\end{array}$ & $\begin{array}{l}\text { Considera i segni } \\
\text { e/o i sintomi dei } \\
\text { pazienti prima di } \\
\text { una diagnosi } \\
\text { definitiva } \\
\text { Con questo metodo } \\
\text { si trattano sintomi } \\
\text { e segni prima di aver } \\
\text { effettuato una diagnos } \\
\text { definitiva, } \\
\text { particolarmente nei } \\
\text { pazienti instabili }\end{array}$ & $\begin{array}{l}\text { Considera una lista } \\
\text { di possibili diagnosi } \\
\text { che non possono } \\
\text { mancare per } \\
\text { determinati sintomi } \\
\text { si }\end{array}$ & $\begin{array}{l}\text { Ricerca iterativa, } \\
\text { incessante e cumulativa } \\
\text { (per sfinimento) di dati } \\
\text { in maniera } \\
\text { indiscriminata. } \\
\text { Viene costruito in maniera } \\
\text { acritica ed afinalistica il } \\
\text { data base del paziente, e, } \\
\text { sull'esuberanza dei dati } \\
\text { raccolti, si inizia il } \\
\text { ragionamento diagnostico } \\
\text { Rientra nell'ambito del } \\
\text { sovra-utilizzo, di } \\
\text { prestazioni inappropriate, } \\
\text { in particolare quelle } \\
\text { diagnostiche, non } \\
\text { giustificata solo dalla } \\
\text { medicina difensiva, ma } \\
\text { anche da uno scarso } \\
\text { impiego del ragionamento } \\
\text { ipotetico-deduttivo }\end{array}$ \\
\hline $\begin{array}{l}\text { Il caso viene correlato } \\
\text { ad una precedente } \\
\text { esperienza }\end{array}$ & $\begin{array}{l}\text { 'se X allora Y', } \\
\text { ma non sempre è } \\
\text { tutto così'semplice: } \\
\text { - l'allineamento fra } \\
\text { due sequenze è un } \\
\text { problema facile se } \\
\text { dispongo di un } \\
\text { algoritmo efficiente, } \\
\text { - l'allineamento } \\
\text { multiplo è un } \\
\text { problema difficile } \\
\text { e (quasi sicuramente } \\
\text { non troverò mai un } \\
\text { algoritmo efficiente }\end{array}$ & $\begin{array}{l}\text { Problem solving per } \\
\text { utilizzare le } \\
\text { conoscenze, } \\
\text { precedenti } \\
\text { per definire le } \\
\text { soluzioni più } \\
\text { adeguate } \\
\text {, } \\
\text { e) }\end{array}$ & $\begin{array}{l}\text { È probabilmente il } \\
\text { più usato in } \\
\text { medicina d'urgenza } \\
\text { rispetto a qualsiasi } \\
\text { altra } \\
\text { specializzazione } \\
\text { medica }\end{array}$ & $\begin{array}{l}\text { Considera le } \\
\text { diagnosi } \\
\text { differenziali } \\
\text { peggiorative e/o } \\
\text { meno comuni }\end{array}$ & $\begin{array}{l}\text { Può comportare un uso } \\
\text { eccessivo delle risorse }\end{array}$ \\
\hline
\end{tabular}


in una precisa categoria nosografica (Tabella 5). ${ }^{16-22}$

Consideriamo di seguito gli aspetti salienti di alcuni degli strumenti potenzialmente disponibili.
Protocolli, punteggi, algoritmi $e$ flow chart

In questo approccio può essere incluso il metodo delle regole di previsione clinica ('clinical prediction

Tabella 3. Segue dalla pagina precedente

\begin{tabular}{|c|c|c|c|c|c|}
\hline Pattern recognition & $\begin{array}{l}\text { Linee guida, flow } \\
\text { charts e algoritmi } \\
\text { (modello } \\
\text { algoritmico- } \\
\text { cook-book) }\end{array}$ & $\begin{array}{l}\text { Metodo ipotetico- } \\
\text { deduttivo }\end{array}$ & $\begin{array}{l}\text { Event driven } \\
\text { (guidato da eventi) }\end{array}$ & $\begin{array}{l}\text { Rule out worst-case } \\
\text { scenario }\end{array}$ & $\begin{array}{l}\text { Metodo exhaustion } \\
\text { (metodo esaustivo) }\end{array}$ \\
\hline $\begin{array}{l}\text { Corrisponde al livello } \\
\text { più basso della gerarchia } \\
\text { decisionale clinica }\end{array}$ & $\begin{array}{l}\text { Metodo rigido, è } \\
\text { necessario } \\
\text { riconoscere il } \\
\text { modello e/o il } \\
\text { contesto ed applicare } \\
\text { le raccomandazioni } \\
\text { Economico, poco } \\
\text { ansiogeno, fa } \\
\text { risparmiare energie }\end{array}$ & $\begin{array}{l}\text { Richiede: } \\
\text { - un'elaborazione } \\
\text { consapevole ed } \\
\text { analitica delle } \\
\text { conoscenze, per } \\
\text { dare origine nuove } \\
\text { soluzioni } \\
\text { - una valutazione } \\
\text { critica dei dati per } \\
\text { interpretare/ } \\
\text { confermare o } \\
\text { respingere le } \\
\text { ipotesi diagnostiche } \\
\text { provvisorie } \\
\text { - un affinamento } \\
\text { delle ipotesi - } \\
\text { diagnostiche con le } \\
\text { informazioni } \\
\text { aggiuntive } \\
\text { - una verifica finale } \\
\text { dell'ipotesi } \\
\text { diagnostica più } \\
\text { probabile }\end{array}$ & $\begin{array}{l}\text { Valuta le possibili } \\
\text { evoluzioni } \\
\text { successive } \\
\text { e considera altre } \\
\text { decisioni in } \\
\text { funzione delle varie } \\
\text { alternative } \\
\text { diagnostiche } \\
\end{array}$ & $\begin{array}{l}\text { Bisogna considerare/ } \\
\text { riconoscere la } \\
\text { possibilità di una } \\
\text { malattia critica } \\
\text { sottesa }\end{array}$ & $\begin{array}{l}\text { Può comportare un } \\
\text { consumo eccessivo di } \\
\text { tempo }\end{array}$ \\
\hline $\begin{array}{l}\text { La maggior parte dei } \\
\text { professionisti richiede } \\
\text { numerose esperienze } \\
\text { precedenti su casi } \\
\text { analoghi }\end{array}$ & $\begin{array}{l}\text { ACLS, ATLS, } \\
\text { Probabilità di } \\
\text { embolia } \\
\text { polmonare e di } \\
\text { trombosi veosa } \\
\text { profonda (TVP) } \\
\text { Linee guida per } \\
\text { singole patologie, } \\
\text { in assenza di } \\
\text { co-morbilità }\end{array}$ & $\begin{array}{l}\text { Richiede maggior } \\
\text { tempo decisionale }\end{array}$ & $\begin{array}{l}\text { Esclude comunque } \\
\text { uno scenario } \\
\text { peggiore }\end{array}$ & & $\begin{array}{l}\text { Può comportare una } \\
\text { dilazione decisionale }\end{array}$ \\
\hline \multirow[t]{2}{*}{$\begin{array}{l}\text { Richiede la } \\
\text { memorizzazione di un } \\
\text { numero critico di } \\
\text { esperienze cliniche } \\
\text { concrete }\end{array}$} & $\begin{array}{l}\text { Limiti: } \\
\text { non valido per i } \\
\text { casi complessi } \\
\text { e multi-patologici }\end{array}$ & $\begin{array}{l}\text { Utile per i medici } \\
\text { inesperti, che hanno } \\
\text { bisogno di tempo } \\
\text { per decidere su casi } \\
\text { non precedentemente } \\
\text { esperiti }\end{array}$ & $\begin{array}{l}\text { Si concentra sulle } \\
\text { azioni di } \\
\text { stabilizzazione, } \\
\text { non sulla diagnosi }\end{array}$ & & $\begin{array}{l}\text { Può comportare una delega } \\
\text { delle responsabilità }\end{array}$ \\
\hline & $\begin{array}{l}\text { Il mondo clinico } \\
\text { reale dei pazienti } \\
\text { non sempre può } \\
\text { essere limitato da } \\
\text { linee guida rigide, } \\
\text { riferite ad una } \\
\text { singola patologia }\end{array}$ & $\begin{array}{l}\text { Utile per i medici } \\
\text { più esperti, che } \\
\text { hanno bisogno di } \\
\text { più tempo per } \\
\text { decidere su casi } \\
\text { particolarmente } \\
\text { complessi }\end{array}$ & $\begin{array}{l}\text { Il metodo aiuta nelle } \\
\text { successive } \\
\text { decisioni } \\
\text { diagnostico- } \\
\text { terapeutiche }\end{array}$ & & $\begin{array}{l}\text { Applicato più } \\
\text { frequentemente da medici } \\
\text { inesperti e principianti, } \\
\text { disorientati, stanchi, } \\
\text { deprivati di sonno, ma } \\
\text { anche per guadagnare } \\
\text { tempo } \\
\text { È un metodo invalidato da } \\
\text { numerosi studi, che } \\
\text { dovrebbe essere } \\
\text { definitivamente } \\
\text { abbandonato }\end{array}$ \\
\hline
\end{tabular}


rules'), di cui alla Tabella 5. I protocolli, le flow chart e gli algoritmi sono utilizzati per semplificare il processo decisionale: la conclusione diagnostica è raggiunta mediante successive scelte tra le possibili relazioni tra uno step e l'altro dell'intero percorso diagnostico-terapeutico. ${ }^{23}$ Gli algoritmi e le flow chart sono strumenti sempre più utilizzati, anche se considerati da alcuni troppo semplicistici. Offrono però il potenziale vantaggio di risparmiare tempo e ansia soprattutto quando i medici devono prendere decisioni rapide in situazioni potenzialmente letali. Per l'impiego ottimale di questi protocolli, i medici devono comprendere bene ed approfondire le conoscenze scientifiche e i riferimenti bibliografici su cui si fondano. Un esempio è dato dagli algoritmi in uso per il trombo-embolismo venoso (esempio score di Wells e score di Geneva). ${ }^{24}$

\section{L'associazione di più sintomi o segni (pattern recognition)}

Il pattern recognition può essere 'trigger'o 'fit' ${ }^{21}$ Nel semplice 'pattern recognition trigger' l'associazione di più sintomi o segni richiama un modello già noto, in grado di far scattare l'ipotesi

Tabella 4. Quale processo decisionale è migliore? Non esiste una risposta univoca.

Dipende dal setting assistenziale (Pronto Soccorso e medicina d'urgenza, Osservazione breve intensiva, Medicina interna, Geriatria, Lungodegenza) e dal contesto organizzativo dell'Ospedale/Reparto

Dipende dal tipo di paziente (critico, stabile, instabile, cronico stabilizzato, terminale)

Dipende dal livello di esperienza, competenza e capacità critica, anche di livello superiore del clinico

Dipende dalla valutazione prognostica

Tabella 5. Strategie rapide e lente del processo decisionale.

STRATEGIE RAPIDE

Diagnosi a colpo d'occhio ('spot'- 'spot eye' 'one shot'diagnosis) correlata a percezioni uditive o visive e da precedenti esperienze del medico: non richiede riflessioni e talora neppure indagini successive

Auto-diagnosi (Self labelling)

Il paziente stesso denunciando il sintomo principale suggerisce una possibile diagnosi, in relazione alle sue precedenti esperienze

Il problema riferito al medico corrisponde alla diagnosi (Presenting complaint)

Il sintomo lamentato dal paziente definisce di per sé la diagnosi (ad es. cefalea, lombalgia, eccetera)

Trigger di riconoscimento (Pattern recognition trigger)

l'associazione di più segni o sintomi evoca una possibile ipotesi diagnostica

\section{STRATEGIE LENTE}

Esclusione limitata di diagnosi possibili (Restricted rule-out)

Viene preso in considerazione un ventaglio di ipotesi diagnostiche escludendo via via quelle meno probabili

Definizione graduale della diagnosi (Stepwise refinement)

A partire dal sintomo, si procede per approfondimenti successivi, aggiungendo via via alcuni dati e se ne scartano altri, fino ad arrivare ad una ipotesi diagnostica fondata

Ragionamento probabilistico

- Deterministico: non vi è incertezza (da certe premesse si generano sempre le stesse conseguenze)

- Stocastico: vengono considerate le variazioni (casuali e non) delle variabili presenti, per evidenziare i possibili risultati in termini di probabilità

prevalenza delle malattie e del valore predittivo di segni, sintomi e dati È il metodo che più di qualsiasi altro si basa su procedimenti scientifici ma per essere applicato correttamente richiede la conoscenza della laboratoristici-strumentali e la capacità di utilizzare il Teorema di Bayes sulla probabilità condizionata. Comporta un uso approssimativo ma efficace della prevalenza stimata di una determinata malattia

Riconoscimento del modello idoneo di sintomi/segni già noti (Pattern recognition fit)

I sintomi ed i segni clinici vengono inquadrati in un contesto globale diagnostico-differenziale (in casi complessi) che richiama altri quadri clinici, oggetto di diagnosi definite proficuamente in precedenza.

Regole di previsione clinica (Clinical prediction rule)

Corrispondono a strumenti matematici che hanno lo scopo di guidare i clinici nel processo decisionale. Sono utilizzati criteri di orientamento diagnostico codificati, ad esempio scala di Wells per la TVP

Capacità di formulare la prognosi

- giudizio soggettivo: previsione clinica di sopravvivenza (ipotesi)

- giudizio attuariale: fattori predittivi di malattia avanzata (strumenti predittivi, punteggi, indici, eccetera, sulla base di studi di ricerca)

Modificata da De Gobbi et al. ${ }^{2}$ 
diagnostica. Ad esempio l'associazione di tachicardia, ansia e dimagrimento può evocare una diagnosi di ipertiroidismo. Nel pattern recognition fit il quadro clinico viene confrontato con esperienze precedentemente diagnosticate dal medico, ma con un perfezionamento del metodo clinico, aggiungendo all'ipotesi iniziale le possibili diagnosi differenziali alternative, riferibili ad ipotesi diagnostiche più complesse. Un tipico esempio dei limiti di un metodo trigger non fit è la 'door-way diagnosis' di un paziente che si agita per un severo dolore al fianco e al dorso. Una diagnosi semplicistica sembrerebbe abbastanza ovvia, inducendo il medico ad una prematura ed errata conclusione diagnostica di colica renale (inquadrabile in un errore del tipo bias di ancoraggio). La presentazione clinica del paziente, con un giudizio clinico complessivo e dei fattori di rischio presenti, potrebbe invece evocare anche un possibile aneurisma dell'aorta addominale complicato da rottura o dissezione, che, se non diagnosticato tempestivamente, potrebbe condurre a morte il paziente. I medici possono essere portati, per impellenza, superficialità ed affaticamento, ad ignorare eventuali ulteriori dati disponibili, in grado di confutare la diagnosi iniziale. Un possibile pregiudizio di conferma, da parte di chi prende una decisione sostenendo strenuamente la propria tesi ed ignorando ipotesi alternative, si compendia in un'erronea, a volte opportunistica, raccolta dei dati, che può in alcuni casi condurre ad esiti devastanti. Il buon medico dovrebbe, invece, pensare ad escludere le ipotesi peggiori per diagnosticare quelle più attendibili.

\section{Escludi l'ipotesi peggiore (rule-out worst case)}

Quando vedo un paziente, particolarmente se instabile o critico, devo domandarmi quale possa essere lo scenario peggiore, senza dimenticare nulla, in riferimento ad ogni sintomo chiave o ad un gruppo di sintomi. Con questo metodo si deve esclude la possibilità che i sintomi del pazienti siano correlate a patologie gravate da un'elevata mortalità. Ad esempio, nel valutare un paziente con dolore toracico acuto il medico dovrebbe innanzitutto escludere condizioni patologiche potenzialmente a rischio di vita, quali una sindrome coronarica o aortica acuta, un'embolia polmonare, un pneumotorace spontaneo o la sindrome di Boerhaave. Questa strategia può essere utile nell'area dell'emergenza, proprio per evitare al medico pericolose sottovalutazioni o omissioni diagnostiche di condizioni potenzialmente letali. Il limite di questo metodo è che i medici, particolarmente se inesperti, possano essere così spaventati dal non riconoscere eventuali sintomi di allarme, tanto da coinvolgere tutto l'ospedale in ipotesi diagnostiche a volte assurde e prive di elementi oggettivi di reale emergenza incombente, con enorme, inutile spreco di risorse. In questa strategia diagnostica si inserisce la grande importanza delle red flags nella pratica clinica, ovvero di situazioni che si presentano con sintomi o segni apparentemente comuni e benigni ma che ad un'indagine più accurata possono sottendere situazioni gravi. Alcuni esempi comuni, ma non per questo da sottovalutare, sono riportati nel Tabella 6.

Il disturbo principale lamentato dal paziente, contrassegnato come red flag o sintomo di allarme, va inquadrato nel contesto complessivo, includendo i fattori di rischio sottesi, le condizioni pre-morbose e/o concomitanti, i disturbi che si presentano con caratteristiche diverse da quelle precedenti o con sintomi nuovi (anche quando aspecifici, riscontrabili in diverse altre patologie), insorti più recentemente. Ad esempio, in caso di cefalea di nuova insorgenza l'acronimo mnemonico SNOOP può essere utile per l'individuazione dei casi a rischio: S: Systemic symptoms, illness, or condition (ad es: febbre, calo ponderale, cancro, gravidanza, stato di immunodeficienza inclusa l'infezione da HIV); $\mathrm{N}$ : Neurologic symptoms or abnormal signs (ad esempio:

Tabella 6. Alcuni esempi di bandiere rosse ('red flags') o sintomi di allarme.

Cefalea: quasi sempre benigna ma è necessario escludere, particolarmente nei casi di recente insorgenza, segni di rigidità nucale (meningite o emorragia sub aracnoidea) e di deficit neurologici (neoplasie, ipertensione endocranica, dissezione di arteria cerebrale, eccetera);

Vertigini: più frequentemente benigne, ne va indagata la possibile origine periferica o centrale; in particolare vanno ricercati segni neurologici di sindrome cerebellare (atassia, Incapacità di eseguire correttamente una sequenza di movimenti fini coordinati, impossibilità di eseguire movimenti rapidi alternati, dismetria, ipotonia, nistagmo, eccetera);

Dolore toracico: spesso riferito al medico di base, nella maggioranza dei casi benigno (aspecifico). Bisogna prestare attenzione ai fattori di rischio, ai dati anamnestici ai segni ed ai sintomi concomitanti, che possano far ipotizzare infarto miocardico, embolia polmonare (angor coeruleus), pneumotorace o sindrome aortica acuta;

Dolore lombare: può sottendere patologie gravi del rachide, come neoplasie o metastasi vertebrali, infezioni (spondilo-discite) o una sindrome della cauda equina. Tali condizioni devono essere escluse dallo screening iniziale, mediante la raccolta dei dati clinici e confermate poi da acquisizioni di imaging; nei pazienti ultracinquantenni a rischio cardio-vascolare deve essere esclusa una rottura/dissezione dell'aorta addominale.

Modificato da Kassirer e Kopelman, $1991 .^{21}$ 
stato confusionale, alterato livello di coscienza, edema della papilla, sintomi o segni neurologici focali, meningismo, convulsioni); $\mathrm{O}$ : Onset is new (soprattutto se in età $>40$ anni) o improvviso (ad es. a rombo di tuono); O: Other associated conditions or features (ad es: trauma cranico, utilizzo di droghe o esposizione a tossici, cefalea al risveglio, peggiora con la manovra di Valsalva o con la tosse, l'esercizio fisico e l'attività sessuale); P: Previous headache history con peggioramento della cefalea o variazione nella gravità, nella frequenza o nelle caratteristiche cliniche degli attacchi. ${ }^{25,26}$

\section{Raccolta ridondante di dati (metodo exhaustion)}

Il metodo exhaustion utilizza una raccolta ridondante di dati del paziente per perseguire la diagnosi più probabile. Tale strategia viene spesso utilizzata dagli studenti ma anche da medici esperti quando eccessivamente stanchi o affaticati o quando il dubbio diagnostico o il rischio di errore è alto. L'evidente svantaggio di questo metodo è che è laborioso e richiede tempo. Talvolta e in condizioni di grave incertezza questo metodo viene opportunisticamente impiegato per rimandare le decisioni ad un tempo successivo o ad altri colleghi che dovranno farsi carico del caso.

\section{Metodo guidato dall'evento (event driven)}

Il metodo guidato dall'evento è un modello clinico tipicamente utilizzato in area dell'emergenza. Un esempio di questa strategia è il trattamento dei sintomi presentati dal caso clinico del nostro paziente (come lo stato ipotensivo), prima di iniziare a stabilire una diagnosi. Tale metodologia, con tutte le riserve del caso, è giustificabile quando si tratta di garantire le funzioni vitali e la sopravvivenza del paziente. Il medico del Pronto Soccorso prova rapidamente a stabilire la pervietà delle vie aeree, cerca un accesso venoso centrale e somministra liquidi e agenti vasopressori per migliorare la pressione arteriosa. Di frequente la diagnosi non è effettuata prima del trasferimento del paziente in terapia intensiva, dato che il medico tende a concentrarsi sulle azioni terapeutiche urgenti prima di focalizzarsi sulle possibilità diagnostiche. La strategia guidata dagli eventi è spesso combinata con quella dell'esclusione dello scenario peggiore, con l'obiettivo di stabilizzare rapidamente i pazienti.

\section{Il metodo riduzionistico}

Il riduzionismo è una filosofia di approccio ai problemi complessi tesa alla loro semplificazione, riferita a modelli teorici minimi sufficienti nel definire gli elementi sostanziali in questione. Tale metodologia è assimilabile al concetto del rasoio di Ockham
('Pluralitas non est ponenda sine necessitate'), ${ }^{27}$ che esclude il bisogno di complicare l'interpretazione dei casi considerando tutte le variabili in gioco. Il metodo riduzionistico si fonda su un teorema - non dimostrato - della razionalità assoluta, per cui il ragionamento clinico può basarsi su molteplici abilità di raccogliere informazioni su una scelta, sulla capacità di elaborare le informazioni estemporaneamente e di trovare la soluzione ottimale al problema da affrontare. ${ }^{28}$ L'approccio riduzionistico può essere utile per l'internista che, di fronte al paziente pluri-patologico, deve estrapolare gli elementi salienti ai fini decisionali.

\section{Conclusioni}

'Vedi gli alberi e non la foresta, detective. Gli alberi, e non la foresta' Da Jack Reacher - La prova decisiva, Film del 2012, Regia di C. McQuarrie

Quale metodo è migliore ai fini del processo decisionale? Nessuna ricerca ha ancora definito quali percorsi possono essere più utili per $\mathrm{i}$ singoli professionisti nei vari setting di cura. Probabilmente la maggior parte dei medici utilizza - anche non consapevolmente -i vari metodi di decisione clinica di tipo intuitivo o razionale/analitico - singolarmente $\mathrm{o}$ in combinazione (metodo duale o binario), a seconda delle varie situazioni cliniche e della complessità dei casi. Qualsiasi strategia decisionale può essere inficiata da errori o da un ragionamento clinico difettoso. I medici devono saper riconoscere le circostanze in cui la loro strategia clinica può risultare imperfetta, proprio per evitare una diagnosi sbagliata; devono essere sempre consapevoli del rischio di una diagnosi effettuata in maniera superficiale e frettolosa, particolarmente nei casi in cui la conclusione diagnostica avviene in una fase precoce di valutazione del paziente, quando non tutti gli elementi necessari ai fini decisionali sono disponibili. In alcuni casi il contesto organizzativo, per situazioni di congestione e di affollamento in ospedale e nei reparti, può indurre a decisioni frettolose, esponendo il medico al rischio di errore. È necessario controllare sempre l'evoluzione del quadro clinico, ricercare nuove informazioni e rivalutare sistematicamente il paziente rispetto ai dati iniziali, inseguendo i propri giustificati dubbi, anche ricorrendo ad un'osservazione breve intensiva, per poter assumere una conclusione diagnostica sulla quale essere in grado ragionevolmente di rendere conto (secondo il principio dell' 'accountability'). L'internista dovrebbe in sostanza essere in grado di applicare le sue conoscenze ed esperienze precedenti, da quelle basilari a quelle più avanzate, per valutare, selezionare e comprendere $\mathrm{i}$ dati, analizzare gli 
elementi salienti, selezionare le priorità, ricercare le co-morbilità attive, sottese e/o silenti e creare ipotesi credibili ai fini di una sintesi diagnostica non erronea. ${ }^{29,30}$ Con una visione globale e multidimensionale, senza fretta né impellenze estrinseche e con una visione dall'alto, ad elicottero, proprio per poter veder la foresta e non solamente gli alberi. Ma anche senza dover ottemperare pedissequamente a linee guida, rinunciando al giudizio critico, particolarmente in casi complessi multi-patologici. Le pressioni sul medico finalizzate ad ottimizzare i costi di gestione e l'efficienza (ad esempio, la durata della degenza che deve essere sempre più breve), a scapito dell'accuratezza, efficacia e qualità delle cure, hanno contribuito ad alimentare una mentalità del tipo prima prova - o anche prescrivi un trattamento -, poi pensa, a detrimento della correttezza della diagnosi, con aumentato rischio di errori. ${ }^{31}$ La difficoltà a formulare la diagnosi a partire dai problemi posti dal paziente passa attraverso la non sempre univoca definizione dello stato di malattia e della sua nosografia. Il giudizio diagnostico appare come un complesso atto immaginativo e creativo, a volte luce che si accende improvvisa, espressione di una conoscenza tacita. ${ }^{32,33}$ Gran parte del procedimento diagnostico - e quindi la decisione terapeutica - è il risultato di una ricerca personale attiva e ponderata. La diagnosi costituisce l'atto intellettualmente più nobile della nostra professione. Il buon medico sa, a partire dai dati anamnestici, dall'esame obiettivo e da una valutazione multidimensionale, anche mediante uno sforzo creativo, individuare i problemi del paziente, formulare ipotesi diagnostiche preliminari $o$ provvisorie, sottoporre queste ultime ai più appropriati controlli servendosi del laboratorio e delle indagini strumentali e, in base ai risultati ottenuti, formulare il giudizio finale. Nella gerarchizzazione delle scelte decisionali, il buon medico deve saper selezionare le priorità e tenere conto del rapporto costo/beneficio nell'adottare ipotesi diagnostiche alternative, in funzione degli elementi disponibili. Il buon medico deve saper rilevare i sintomi, valutarli, coordinarli e ricavarne la diagnosi, per poi prescrivere la terapia. Compiere queste operazioni, e compierle bene, non sempre è facile. Il cosiddetto occhio clinico (ovvero il buonsenso) non è che la loro risultante, che emerge quasi istantaneamente in un intelletto ben dotato dalla natura e ben educato dall'istruzione e dall'esperienza. Tutto ciò, nell'accezione moderna, si esprime, nella pratica reale, nelle capacità del buon medico di assumere le decisioni in base a una valutazione ponderata, non influenzata da conflitto di interesse alcuno, alle conoscenze scientifiche e alle prove disponibili, e all'esperienza, al contesto reale oggettivo, tenendo conto delle scelte e dei diritti del paziente. È necessario disporre dei tempi dovuti. In sostanza, l'elogio della lentezza, del pensiero critico e dell'intelligenza emotiva del medico internista, nell'interesse del paziente, richiamano il dovere del medico a non essere mai frettoloso, a saper ascoltare il paziente, valutandone la complessità, rispettandone i tempi e considerandone il decorso clinico, anche in termini di continuità assistenziale. Il metodo clinico deve continuare a far parte fondante della competenza del clinico: esso va insegnato a tutti i medici che intendono essere a contatto con gli ammalati, affinché non vengano fuorviati dalla falsa idea che la tecnologia possa bastare. Tali principi fondanti, gradevolmente espressi da Vito Cagli, fanno parte della mentalità degli Internisti, che certamente vi ritroveranno i valori che ispirano la loro pratica quotidiana, in una passione che deve aiutarci a essere uomini che vivano per la loro scienza e non come pallidi burocrati attenti a timbrare il cartellino all'ora giusta o a cercare il DRG più conveniente. Un medico senza passione non sente la vita né la morte; forse può scorgere la malattia, ma non vedrà mai l'ammalato. ${ }^{27,34}$ Decidere è la scelta d'intraprendere un'azione tra più alternative e rappresenta un elemento essenziale di libertà. Decidere in maniera razionale significa conoscere le opzioni disponibili e le conseguenze che possono scaturire dalla nostra scelta. Le linee guida (LG), su cui si fondano oggi, anche per riferimenti normativi, le valutazioni sulla qualità dell'assistenza prestata e le relative responsabilità professionali, rappresentano per il medico un'opportunità per ottimizzare i processi di cura. Purtroppo le LG presentano diverse limitazioni. Sono troppo spesso incentrate sulle singole patologie e non sono mirate alle peculiarità dell'ammalato. In Medicina Interna i pazienti raramente hanno singole malattie e poche, se non nessuna, LG sono in grado di supportare il medico nel gestire casi complessi e garantire la personalizzazione delle cure. Le LG sono, a volte, anche troppo minuziose, descrivendo ogni possibile intervento che potrebbe essere appropriato ed ottimale per un paziente con una singola malattia, fino al paradosso di indurre, specialmente nei pazienti pluri-patologici, una serie innumerevole di accertamenti diagnostici e di prescrizioni, ${ }^{35}$ senza facilitare decisioni univoche ed orientate da parte del medico. La scienza clinica e l'esperienza del medico hanno entrambe un ruolo fondamentale in Medicina Interna e devono necessariamente essere complementari. Non é possibile sostituire l'una con l'altra, privilegiando tecniche di calcolo quantitativostatistico rispetto alla valutazione della rilevanza clinica dei dati, in base alla capacità d'intuizione e di ragionamento del medico, senza incorrere in errori di tipo dogmatico e manicheo. ${ }^{36}$ Ribadiamo, in sostanza, gli elementi salienti già più volte esplicitati a partire 
dall'originale declinazione della 'mission' della FADOI: i) Siamo convinti che la buona metodologia clinica resta un valido strumento per la crescita razionale ed equilibrata degli ospedali; ii) Sostituire il ragionamento clinico con l'immediato ricorso alla diagnosi strumentale vuol dire lasciar crescere una visione fortemente tecnologica della medicina, ovvero: iii) costruire una medicina insostenibile, sia dal punto di vista organizzativo che sul piano economico. $^{37}$

\section{Post scriptum}

Nel manoscritto abbiamo volute mantenere diversi termini inglesi originali 'virgolettati' o in corsivo non per una auspicata anglofilia o ripudio della lingua italiana. La lingua inglese domina la letteratura scientifica medica di qualità. Abbiamo voluto facilitare la lettura delle principali parole chiave sul tema e di molti articoli di riferimento, con un glossario pertinente all'argomento affrontato.

\section{Bibliografia}

1. Diagnosis. The American Heritage Dictionary of the English Language. $4^{\text {th }}$ Edition ed: Houghton Mifflin Company; 2003.

2. Sackett DL et al. Evidence based medicine: what it is and what it isn't, BMJ 1996, 312: 71.

3. Descovich C, Nardi R, Ligabue A et al. Inappropriate emergency laboratory test ordering: defensive or peer evidence shared based medicine? Italian Journal of Medicine 2013, 2/4: 13-22.

4. McGrath M. https://leanforward.hms.harvard.edu/2017 /10/24/clinical-reasoning-storing-stories-to-narrow-thediagnosis/, 24 ottobre 2017.

5. Staitieh BS, Saghafi R, Kempker JA, Schulman DA. Teaching a Hypothesis-driven Physical Diagnosis Curriculum to Pulmonary Fellows Improves Performance of First-Year Medical Students, Ann Am Thorac Soc. 2016, 13/4: 489-494.

6. Yudkowsky R, Otaki J, Lowenstein T, Riddle J, Nishigori H, Bordage G. A hypothesis-driven physical examination learning and assessment procedure for medical students: initial validity evidence, Med Educ 2009, 43: 729-740.

7. Yudkowsky R, Downing S, Klamen D, Valaski M, Eulenberg B, Popa M. Assessing the head-to-toe physical examination skills of medical students, Med Teach 2004; 26: 415-419.

8. La Combe MA. On bedside teaching, Ann Intern Med 1997; 126: 217-220.

9. Carver College of Medicine, University of OHIO, Dept of Internal Medicine, Clinical and Diagnostic Reasoning, https://medicine.uiowa.edu/internalmedicine/education/m aster-clinician- program/students/clinical-and-diagnosticreasoning).

10. Rosselli A. Medicina d'emergenza-urgenza: dal modello cognitivo all'approccio operativo e alla strategia della formazione. Una premessa all'avvio delle Scuole di specialità, XXIX Seminario dei Laghi. L'educazione alla salute, Gardone Riviera, 12 ottobre 2007.

11. Redelmeier DA. Improving patient care. The cognitive psychology of missed diagnoses, Ann Intern Med 2005, 138/1:78

12. Rapezzi C, Ferrari R, Branzi A. White coats and fingerprints: diagnostic reasoning in medicine and investigative methods of fictional detectives, BMJ 2005; 331: 1491-1494.

13. Harpartap S, Carpenter C. Clinical Decision making: Opening the Black Box of Cognitive Reasoning. Annals of Emergency Medicine, 2006, 48/6: 713-719.

14. Churches A. Bloom's Taxonomy Blooms Digitally, 2008; http://www.techlearning.com/showArticle.php? articleID $=196605124$

15. De Gobbi R. Le trappole del ragionamento medico: ecco cosa ci insegna la psicologia cognitiva, http://www. pillole.org/public/aspnuke/news.asp? $\mathrm{id}=7067 \& \mathrm{sid}=$ 279574456

16. Caimi V, Tombesi M. Medicina Generale, UTET Editore, Torino 2003; Scandellari C, La Diagnosi Clinica Milano: Masson Editore 2005.

17. Scandellari C. La Diagnosi Clinica Milano: Masson Editore 2005.

18. Federspil G. I fondamenti del metodo in medicina clinica e sperimentale. Padova: Piccin Editore 1980.

19. Heneghan C, Glasziou P, Thompson M. Diagnostic strategies used in primary care, BMJ 2009; 338b: 946.

20. Norman G, Building on experience-the development of clinical reasoning, N Engl J Med 2006; 355: 2251-2252.

21. Kassirer JP, Kopelman RI. Learning Clinical Reasoning. Philadelphia, PA: Lippincott Williams \& Wilkins; 1991.

22. De Gobbi R, Di Marco L, Fassina R. Strategie diagnostiche in Medicina Generale, Società Italiana Medicina Generale 2011; 6: 3-8.

23. Serio A, Cicala M, La metodologia clinica nella formazione del medico, MEDIC 2013; 21/1: 75-81.

24. 2014 ESC Guidelines on the diagnosis and management of acute pulmonary embolism, European Heart Journal 2014, 35: 3033-3080.

25. Dodick D. Pearls: headache, Semin Neurol 2010, 30: 74-81.

26. Martin VT. The diagnostic evaluation of secondary headache disorders, Headache 2011, 51/2: 346-352.

27. Novacula Occami, William of Ockham-1287-1347.

28. Scandellari C, Medicina degli schiavi e dei liberi, Tutor 2014, 3: 6-11.

29. Bloom BS, Engelhart M, Furst EJ, Hill WH, Krathwohl DR. Taxonomy of educational objectives: The classification of educational goals. Handbook I: Cognitive domain. New York: David McKay Company, 1956.

30. Anderson LW, Krathwohl D. A Taxonomy for Learning, Teaching and Assessing: a Revision of Bloom's Taxonomy of Educational Objectives. Longman, New York, 2001.

31. Simpkin AL, Vyas JM, Armstrong KA. Diagnostic medicine Training. Ann Intern Med, 2017;167:507-508. [Epub aheadReasoning: An Endangered Competency in Internal Med of print 12 September 2017]. doi: https://doi.org/10.7326/M17-0163).

32. Cagli V. Elogio del metodo clinico. Mutamenti e problemi della Medicina al letto del malato, Armando Ed, Roma 1997. 
33. Cagli V. La crisi della diagnosi, Armando Ed. Roma 2007.

34. Nardi R. Recensione del volume di Vito Cagli "La crisi della diagnosi”, Italian Journal of Medicine 2007; 1/3: 80.

35. Boyd CM, Darer J, Boult DJ, Fried LP, Wu AW. Clinical practice guidelines and quality of care for older patients with multiple comorbid diseases: implications for pay for performance, JAMA 2005, 294/6: 716-724.

36. Nardi R et al. Medicina Interna, paziente complesso, EBM e le non evidenze, It J Med 2009, 3: 191-200.

37. Iori I. La mission di FADOI, http://www.fadoi.org/fadoi _chi_siamo/ 


\title{
I difetti del ragionamento clinico come causa di errori diagnostici
}

\author{
Micaela La Regina, ${ }^{1}$ Francesco Orlandini ${ }^{2}$ \\ ${ }^{1}$ S.S. Risk Management, Azienda Sociosanitaria Ligure 5, La Spezia; ${ }^{2}$ Direttore Sanitario ASL4 Li-guria, Chiavari (GE), Italia
}

\section{Introduzione}

I difetti del ragionamento clinico possono determinare sia errori di diagnosi che di trattamento. ${ }^{1}$

Secondo l'American National Academy of Medicine (nota precedentemente come Institute of Medicine), un errore diagnostico è un fallimento nello (i) stabilire una spiegazione accurata e tempestiva dei problemi di salute del paziente o nel (ii) comunicare tale spiegazione al paziente. Questa definizione include: diagnosi errate, ritardate o omesse. ${ }^{2}$

L'incidenza degli errori diagnostici varia in base alla definizione utilizzata, alla disciplina in esame e alla metodologia di ricerca. Ad esempio, 1 diagnosi su 10 è errata (negli studi basati su secret shoppers), 1 autopsia ogni 10-20 identifica importanti discrepanze diagnostiche, 1 paziente su 3 è stato vittima di un errore diagnostico (secondo survey effettuate su pazienti), 1 paziente su 20 sperimenterà ogni anno un errore diagnostico (secondo gli studi di revisione di cartelle cliniche). Gli errori diagnostici sono la causa più comune di reclami per negligenza ${ }^{3}$ e circa la metà dei medici ammette almeno un errore diagnostico al mese e percepisce gli errori diagnostici come i più pericolosi (secondo survey condotte su medici). ${ }^{4}$

Le malattie più comunemente oggetto di omissioni o ritardi diagnostici sono: l'embolia polmonare e le reazione avverse o overdose da farmaci $(2,5 \%)$, il tumore polmonare $(3,9 \%)$, il carcinoma del colon-retto $(3,6 \%)$, la sindrome coronarica acuta $(3,1 \%)$, il carcinoma mammario $(2,9 \%)$ e l'ictus $(2,6 \%) .{ }^{5} \mathrm{Ad}$ ogni modo, i medici sopravvalutano le loro capacità diagnostiche: solo il $10 \%$ dei medici ammette di aver

Corrispondente: Micaela La Regina, S.S. Risk Management, Azienda Sociosanitaria Ligure 5, via Fazio 30, 19121 La Spezia, Italia.

Tel.: 0187/533782. E-mail: micaela.laregina@as15.liguria.it

Articolo pubblicato secondo la Creative Commons Attribution NonCommercial 4.0 License (CC BY-NC 4.0).

${ }^{\circ}$ Copyright: the Author(s), 2020

Licensee PAGEPress, Italy

QUADERNI - Italian Journal of Medicine 2020; 8(1):31-36 commesso almeno un errore diagnostico nell'ultimo anno, ${ }^{6}$ ma fino al $40 \%$ delle diagnosi di cui i clinici erano certi si è rivelato sbagliato all'autopsia. ${ }^{7}$ Anche quando la diagnosi è corretta, possono insorgere errori nella scelta del trattamento. Infatti, 1 paziente su 2 affetti da malattie acute o croniche non riceve terapie evidence-based e 1 ogni 3-5 riceve farmaci o indagini non necessari e/o potenzialmente pericolosi. ${ }^{1}$

Un terzo degli errori deriva da deficit nell'esecuzione (dimenticanze, lapsus o sviste nella gestione appropriata di casi correttamente diagnosticati), ma quasi la metà sono errori di ragionamento o di decisione (scarsa capacità di raccogliere o sintetizzare le informazioni cliniche, decidere o agire sulla base di esse).

Il $25 \%$ di tali errori comportano morte o invalidità permanente e almeno tre quarti sono altamente prevenibili. ${ }^{4}$

Una pietra miliare della ricerca sugli errori di ragionamento clinico in medicina interna è il lavoro di Graber et al. ${ }^{8}$ Essi hanno analizzato 100 casi e raggruppato gli errori diagnostici rilevati in 3 categorie: i) errori senza colpa (in caso di presentazione subdola o atipica della malattia o paziente non collaborante) $7 \%$; ii) errori di sistema (riconducibili a guasti tecnici, problemi alle apparecchiature o difetti organizzativi) $19 \%$; iii) errori cognitivi (deficit di conoscenza, raccolta o sintesi di dati) $28 \%$.

$\mathrm{Nel} 46 \%$ dei casi si riscontrava addirittura la coesistenza di errori cognitivi e errori di sistema. Oltre a ciò, le diagnosi errate erano associate prevalentemente a fattori contribuenti cognitivi ( $92 \%$ vs 50\%), mentre i ritardi diagnostici a fattori di sistema ( $89 \%$ vs 36\%). Nei casi in cui la diagnosi autoptica non coincideva con la diagnosi clinica risultavano predominanti i fattori cognitivi (90\% vs 10\%). Nel complesso, gli autori identificavano 228 fattori contribuenti di sistema e 320 cognitivi, con una media di 5.9 fattori per caso. Fra i fattori contribuenti cognitivi, un'erronea raccolta (14\%) o sintesi $(83 \%)$ dei dati erano più frequenti di un deficit di conoscenza $(3 \%)^{8}$.

Di fatto, esistono due tipi di ragionamento clinico: analitico e non analitico. Il ragionamento analitico, denominato anche ipotetico-deduttivo, è utilizzato comunemente dai medici più giovani $\mathrm{o}$ in casi con 
manifestazioni insolite o meno familiari, ed è basato su liste di diagnosi differenziali e la conseguente raccolta di informazioni per validare o escludere tali diagnosi. Il ragionamento non analitico è più veloce, basato su scorciatoie mentali, dette euristiche, quali massime, regole empiriche, e sul riconoscimento di modelli (patterns). In pratica, il medico confronta automaticamente i sintomi/segni del paziente che ha davanti con quelli di casi incontrati precedentemente nella pratica clinica o conosciuti attraverso lo studio, e arriva alla diagnosi in pochi secondi. ${ }^{9}$ Un tipo di ragionamento non esclude l'altro; entrambi possono essere mutualmente utilizzati nello stesso paziente. Nessuno di essi è immune da errori. Se le euristiche e il riconoscimento dei pattern sono efficienti e accurati in molte situazioni, possono anche favorire talora degli errori, quando ad esempio il caso del paziente non calza alla perfezione con il pattern atteso a causa di una presentazione atipica, dell'evoluzione della patologia o di comorbidità. ${ }^{10}$ Un altro tallone di Achille del ragionamento non analitico sono i bias, procedimenti basati su percezioni, pregiudizi o ideologie, estranei al pensiero razionale. I bias possono essere distinti in interni o esterni ai clinici ${ }^{1}$ o cognitivi e affettivi ${ }^{11}$ (Tabella 1).

Tabella 1. Bias ed euristiche del ragionamento clinico: esempi e strategie correttive.

\begin{tabular}{llll}
\hline Bias & Descrizione & Esempio & Strategiacorrettiva \\
\hline Anchoring & $\begin{array}{l}\text { Tendenza a fermarsi alla prima } \\
\text { impressione e a non considerare } \\
\text { tutte le informazioni disponibili }\end{array}$ & $\begin{array}{l}\text { Un medico ha diagnosticato una } \\
\text { meningite vitale invece di una }\end{array}$ & $\begin{array}{l}\text { Andare oltre la propria diagnosi preferita } \\
\text { e la prima impressione }\end{array}$ \\
& $\begin{array}{l}\text { solo sulla febbre elevate e il dolore caso di acquisizione di nuovi dati o } \\
\text { al collo, e ignorando che il dolore al decorso inatteso } \\
\text { collo peggiora non solo con la }\end{array}$ & \\
& flessione ma anche con la & palpazione e ignorando la precedente \\
& ferita all'avambraccio &
\end{tabular}

\begin{tabular}{|c|c|c|c|}
\hline Availability & $\begin{array}{l}\text { Tendenza ad accettare la prima } \\
\text { diagnosi che viene in mente a } \\
\text { causa di una recente } \\
\text { osservazione piuttosto che } \\
\text { considerare la prevalenza e } \\
\text { l'incidenza di tale diagnosi }\end{array}$ & $\begin{array}{l}\text { Il medico ha formulato una diagnosi } \\
\text { di meningite virale anziché di } \\
\text { osteomielite cervicale, dal momento } \\
\text { che aveva appena diagnosticato un } \\
\text { altro caso di meningite virale }\end{array}$ & $\begin{array}{l}\text { Considerare sempre l'incidenza e la } \\
\text { prevalenza delle diverse ipotesi } \\
\text { diagnostiche }\end{array}$ \\
\hline Confirmation & $\begin{array}{l}\text { Cercare solo segni e sintomi } \\
\text { che confermano la propria } \\
\text { ipotesi preferita o interpretare } \\
\text { i risultati clinici solo per } \\
\text { supportare tale ipotesi, senza } \\
\text { cercare o ignorando evidenze } \\
\text { contrarie }\end{array}$ & $\begin{array}{l}\text { Il medico ha diagnosticato } \\
\text { un'eruzione cutanea sotto l'ascella } \\
\text { di un paziente diabetico come } \\
\text { intertrigine omettendo la diagnosi di } \\
\text { eritema migrante da malattia di } \\
\text { Lyme }\end{array}$ & $\begin{array}{l}\text { Utilizzare uno strumento oggettivo come } \\
\text { una checklist per la diagnostica } \\
\text { differenziale, per verificare se la } \\
\text { diagnosi correlate con i risultati dei test }\end{array}$ \\
\hline $\begin{array}{l}\text { Diagnosis momentum } \\
\text { bias }\end{array}$ & $\begin{array}{l}\text { Considerare definita una diagnosi } \\
\text { senza prove, ma solo a causa } \\
\text { di un'etichetta applicata al primo } \\
\text { contatto e trasmessa da tutte le } \\
\text { persone che si sono prese cura } \\
\text { del paziente }\end{array}$ & $\begin{array}{l}\text { Il medico ha attribuito alla } \\
\text { sindrome da astinenza da alcol } \\
\text { l'agitazione psicomotoria di un } \\
\text { paziente identificata come alcolista, } \\
\text { non riconoscendo un quadro di } \\
\text { sepsi grave }\end{array}$ & $\begin{array}{l}\text { Rivedere criticamente le diagnosi } \\
\text { formulate da altri e cercare prove a } \\
\text { supporto di esse }\end{array}$ \\
\hline Framing* & $\begin{array}{l}\text { Scegliere un'opzione in base al } \\
\text { fatto che gli si sia presentata con } \\
\text { connotazioni positive o negative } \\
\text { o dietro influenza del contesto }\end{array}$ & $\begin{array}{l}\text { Il medico può decidere di richiedere } \\
\text { una TAC cranica nello stesso paziente } \\
\text { più spesso se è stata presentata come } \\
\text { associata al } 90 \% \text { di veri positivi } \\
\text { che al } 10 \% \text { di falsi negativi } \\
\text { Il medico può diagnosticare più } \\
\text { facilmente un aneurisma addominale } \\
\text { rotto in pronto soccorso rispetto a un } \\
\text { ambulatorio }\end{array}$ & Cambiare prospettiva \\
\hline Gambler's fallacy & $\begin{array}{l}\text { Ritenere una diagnosi meno } \\
\text { probabile, solo perchè si è } \\
\text { presentata diversi pazienti } \\
\text { precedenti }\end{array}$ & $\begin{array}{l}\text { Il medico ha mancato una diagnosi di } \\
\text { embolia polmonare dal momento che } \\
\text { aveva diagnosticato } 4 \text { casi di embolia } \\
\text { polmonare nell'ultima settimana }\end{array}$ & $\begin{array}{l}\text { Considerare sempre la probabilità } \\
\text { pre-test }\end{array}$ \\
\hline Multiple alternative bias & $\begin{array}{l}\text { Ridurre la diagnosi differenziale } \\
\text { a poche ipotesi diagnostiche più } \\
\text { familiari, quando sono disponibili } \\
\text { più opzioni }\end{array}$ & $\begin{array}{l}\text { Il medico ha omesso una rara diagnosi } \\
\text { di febbre mediterranea familiare e } \\
\text { ha sottoposto il paziente all'intervento } \\
\text { chirurgico per appendicite }\end{array}$ & $\begin{array}{l}\text { Utilizzare uno strumento oggettivo come } \\
\text { una checklist per la diagnostica } \\
\text { differenziale, per verificare se la } \\
\text { diagnosi correlate con i risultati dei test }\end{array}$ \\
\hline
\end{tabular}


Tabella 1. Segue dalla pagina precedente

\begin{tabular}{|c|c|c|c|}
\hline Bias & Descrizione & Esempio & Strategiacorrettiva \\
\hline Outcome bias & $\begin{array}{l}\text { Optare per la diagnosi associata } \\
\text { al miglior risultato, valorizzando } \\
\text { la speranza del medico rispetto } \\
\text { ai dati clinici }\end{array}$ & $\begin{array}{l}\text { Il medico ha interpretato un nodulo } \\
\text { polmonarecome benigno, invece di } \\
\text { ordinare ulteriori indagini }\end{array}$ & \\
\hline $\begin{array}{l}\text { Frequency gambling } \\
\text { bias and Worst-case bias }\end{array}$ & $\begin{array}{l}\text { In presenza di un quadro clinico } \\
\text { ambiguo, optare per una diagnosi } \\
\text { benigna, assumendo che le } \\
\text { malattie benigne siano più comuni. } \\
\text { È l'opposto del bias del caso } \\
\text { peggiore }\end{array}$ & $\begin{array}{l}\text { Il medico ha interpretato la } \\
\text { poliglobulia come reattiva piuttosto } \\
\text { che come disturbo emoproliferativo } \\
\text { in un fumatore accanito }\end{array}$ & $\begin{array}{l}\text { Ampliare la storia clinica per trovare } \\
\text { altre cause o associazioni }\end{array}$ \\
\hline $\begin{array}{l}\text { Posterior probability } \\
\text { error }\end{array}$ & $\begin{array}{l}\text { Assumere che un paziente che } \\
\text { ha gli stessi sintomi della volta } \\
\text { precedente, ha la stessa malattia }\end{array}$ & $\begin{array}{l}\text { Il medico ha diagnosticato } \\
\text { l'insufficienza cardiaca invece } \\
\text { dell'embolia polmonare in un } \\
\text { paziente che presentava dispnea e } \\
\text { ricoveri ospedalieri ripetuti per } \\
\text { insufficienza cardiaca }\end{array}$ & $\begin{array}{l}\text { Utilizzare una check-list per la } \\
\text { diagnostica differenziale e la regola } \\
\text { dell'esclusione dello scenario peggiore } \\
\text { Considerare sempre l'incidenza e la } \\
\text { prevalenza delle diverse ipotesi } \\
\text { diagnostiche } \\
\text { Utilizzare una check-list per la } \\
\text { diagnostica differenziale e la regola } \\
\text { dell'esclusione dello scenario peggiore } \\
\text { Considerare sempre l'incidenza e la } \\
\text { prevalenza delle diverse ipotesi } \\
\text { diagnostiche }\end{array}$ \\
\hline
\end{tabular}

\begin{tabular}{|c|c|c|c|}
\hline Search satisfying bias & $\begin{array}{l}\text { Smettere di cercare le diagnosi } \\
\text { secondarie, dopo aver formulato } \\
\text { la diagnosi principale, perdendo } \\
\text { in tal modo comorbidità, } \\
\text { complicanze e diagnosi secondarie }\end{array}$ & $\begin{array}{l}\text { Attribuire alla cardiopatia } \\
\text { ipertensiva la fibrillazione atriale che } \\
\text { si è verificata in un paziente iperteso, } \\
\text { senza considerare un possibile } \\
\text { ipertiroidismo }\end{array}$ & \\
\hline Sunk cost bias* & $\begin{array}{l}\text { La tendenza a perseguire una linea } \\
\text { di condotta, anche dopo che si è } \\
\text { dimostrata non ottimale, perché } \\
\text { sono state investite delle risorse } \\
\text { in tale linea di condotta }\end{array}$ & $\begin{array}{l}\text { Il medico ha continuato a cercare un } \\
\text { cancro in un paziente che lamentava } \\
\text { astenia, anche se le indagini erano } \\
\text { ripetutamente negative } \\
\text { Non aggrapparti a un errore solo } \\
\text { perché hai passato molto tempo } \\
\text { a farlo Aubrey De Graf }\end{array}$ & \\
\hline Visceral bias & $\begin{array}{l}\text { Optare per una diagnosi } \\
\text { facendosi guidare dalle emozioni }\end{array}$ & $\begin{array}{l}\text { Il medico ha attribuito una anemia } \\
\text { sideropenica all'ipermenorrea in una } \\
\text { paziente sua coetanea, senza } \\
\text { considerare eventuali malattie } \\
\text { intestinali maligne }\end{array}$ & \\
\hline Commission bias & $\begin{array}{l}\text { Tendenza a fare qualcosa anche } \\
\text { se non è supportata da evidenze } \\
\text { robuste o può di fatto causare } \\
\text { danno }\end{array}$ & $\begin{array}{l}\text { Il medico ha accondisceso alla } \\
\text { richiesta di eseguire una puntura } \\
\text { lombare, avanzata dai familiari di } \\
\text { una ragazza di } 18 \text { anni con febbre } \\
\text { e cefalea, nonostante la conta dei } \\
\text { neutrofili fosse normale. } \\
\text { Successivamente la paziente ha } \\
\text { sviluppato una severa cefalea } \\
\text { post-puntura lombare che ne ha } \\
\text { richiesto la ospedalizzazione }\end{array}$ & $\begin{array}{l}\text { Considerare sempre le evidenze } \\
\text { Bilanciare benefici e rischi }\end{array}$ \\
\hline Premature closure & $\begin{array}{l}\text { Smettere di raccogliere } \\
\text { informazioni, dopo aver } \\
\text { raggiunto una diagnosi plausibile }\end{array}$ & $\begin{array}{l}\text { Il radiologo non ha visto una } \\
\text { seconda frattura, dopo aver } \\
\text { identificato la prima }\end{array}$ & $\begin{array}{l}\text { Rivedere il caso, richiedere altre } \\
\text { opinioni (es. seconda opinione) e } \\
\text { consultare risorse oggettive (es. la } \\
\text { revisione da parte di un ortopedico } \\
\text { potrebbe rilevare una frattura } \\
\text { concomitante) }\end{array}$ \\
\hline Representativeness bias & $\begin{array}{l}\text { Formulare una diagnosi tenendo } \\
\text { conto solo delle manifestazioni } \\
\text { tipiche di una patologia }\end{array}$ & $\begin{array}{l}\text { Il medico non ha riconosciuto un } \\
\text { infarto miocardico che si è } \\
\text { presentato con nausea e vomito }\end{array}$ & $\begin{array}{l}\text { Considerare le manifestazioni atipiche } \\
\text { soprattutto nelle donne }\end{array}$ \\
\hline Extrapolation bias* & $\begin{array}{l}\text { Estendere le esperienze e i risultati } \\
\text { degli studi clinici a gruppi di } \\
\text { pazienti nei quali gli interventi } \\
\text { considerati (es. test) non sono } \\
\text { stati adeguatamente valutati }\end{array}$ & $\begin{array}{l}\text { Il medico ha richiesto una TC per } \\
\text { escludere una syndrome coronarica } \\
\text { acuta in un paziente con un } \\
\text { pregresso bypass aortocoronarico }\end{array}$ & $\begin{array}{l}\text { Utilizzare i test per le indicazioni } \\
\text { previste }\end{array}$ \\
\hline
\end{tabular}

*Questi bias possono influenzare non solo il processo diagnostico, ma anche le scelte relative al trattamento. 
I fallimenti del ragionamento analitico derivano più spesso dal non seguire regole diagnostiche appropriate $\mathrm{e}$ includono: perdita di informazioni chiave, inadeguata revisione di dati esistenti, deficit di conoscenze scientifiche, mancanza di competenze nella pratica clinica basata sull'evidenza e nel processo decisionale, errata considerazione del valore dei test diagnostici, scarsa supervisione del ragionamento non analitico da parte di quello analitico. ${ }^{10}$ Infine, un ambiente di lavoro rumoroso, frequenti interruzioni, eccessivo carico di lavoro, stanchezza e pressioni temporali possono compromettere l'efficienza del ragionamento clinico. ${ }^{11}$

La HealthResearch \& Educational Trust (HRET), l'Hospital Improvement Innovation Network (HIIN) team e la Society to Improve Diagnosis in Medicine $(\mathrm{SIDM})^{12}$ hanno pubblicato un documento dal titolo Diagnostic error - Change Package che contiene un insieme di strategie e concetti che ogni ospedale dovrebbe implementare (migliorare l'efficacia del lavoro di gruppo e l'affidabilità del processo diagnostico, coinvolgere pazienti e caregiver, potenziare $\mathrm{i}$ sistemi di reporting and learning $\mathrm{e}$ ottimizzare le performance cognitive dei medici). ${ }^{12} \mathrm{~A}$ questo scopo sono disponibili numerosi strumenti:

- checklist per il processo diagnostico come la CATCH (Comprehensive history and physical exam: accurata storia clinica e esame obiettivo, Alternate explanations: spiegazioni alternative del quadro clinico, Take a diagnostic time out to be certain: prendersi una pausa di riflessione per verificare la diagnosi; Consider critical diagnoses not be missed: considerare le diagnosi critiche che non devono essere omesse, Help if needed: chiedere aiuto, se necessario) $;{ }^{13}$

- strumenti mnemonici di supporto al processo decisionale come la checklist VITAMIN CC \& D (cause Vascolari, Infettive e Tossiche,
Traumatiche, Autoimmuni, Metaboliche, Idiopatiche e iatrogene, Neoplastiche, Congenite, disturbi di Conversione e malattie Degenerative);

- liste di sintomi e segni di allarme (Red flags);

- sistemi elettronici di supporto alla decisione clinica, come il sistema Isabel, che si è risultato associato con i migliori tassi di accuratezza diagnostica $; 3$

- domande per neutralizzare i bias (Tabella 2); ${ }^{14}$

- pratica clinica riflessiva che si può realizzare con una delle seguenti strategie: i) l'esperienza della palla di cristallo: ${ }^{12}$ fermarsi e chiedersi: se la mia diagnosi fosse sbagliata, quali alternative dovrei considerare? ii) la regola dell'esclusione dello scenario peggiore: ${ }^{12}$ escludere prima le diagnosi più gravi; iii) la Sfida tra il Team Blue e Rosso, ${ }^{15}$ derivata dal settore militare, è un metodo sicuro per migliorare la capacità decisionale in situazioni cliniche complesse. Lo staff viene diviso in due team: il Team Blu raccoglie la storia clinica, riassume i punti salienti e genera delle ipotesi diagnostiche e il Team Rosso che fa da revisore indipendente, sottoponendo a revisione critica il quadro clinico presentato e identificando diagnosi alternative a quelle presentate dal team blu; iv) Take 2 - think, $d o^{15}$ è una strategia progettata per migliorare la consapevolezza e il riconoscimento di potenziali errori e ridurre la morbilità e la mortalità di diagnosi errate, mancate o ritardate. Letteralmente, significa Prenditi 2 minuti per porre la diagnosi, per verificare, cioè, se ci sono situazioni che richiedono un approfondimento o una rivalutazione della diagnosi (Momento think) e agire (Momento do). È necessario uno sguardo più attento se il medico è affamato, arrabbiato, in ritardo o stanco (situazioni riassunte nell' acronimo HALT: hungry, angry, late, tired), a rischio di bias

Tabella 2. De-biasing questions.

\begin{tabular}{|c|c|}
\hline Situazioni ad alto rischio & Domande \\
\hline Passaggio di consegne & Questo paziente mi è stato passato in consegna da un altro turno? \\
\hline $\begin{array}{l}\text { Influenze esterne } \\
\text { Eccessiva fiducia in collaboratori e colleghi }\end{array}$ & $\begin{array}{l}\text { La diagnosi mi è stata suggerita, direttamente o indirettamente, dal paziente, da un } \\
\text { infermiere o da un altro medico? }\end{array}$ \\
\hline Eccessiva fiducia in se stessi & Ho scelto la prima diagnosi che mi è venuta in mente? \\
\hline Chiusura prematura & Ho considerate ogni organo e apparato? \\
\hline Pregiudizio o identificazione & $\begin{array}{l}\text { Ho qualche pregiudizio verso questo paziente?Ho qualcosa in comune con questo } \\
\text { paziente? }\end{array}$ \\
\hline Ambiente rumoroso e/o affollato & Ho subito interruzioni o distrazioni durante la valutazione di questo paziente? \\
\hline Stanchezza personale & Ero stanco o assonnato durante la valutazione di questo paziente? \\
\hline Sovraccarico cognitivo & Sono troppo oberato dal punto di vista cognitivo? \\
\hline Situazione stereotipata & Sto stereotipando questo paziente? \\
\hline Eccessivo carico di lavoro, pressioni temporali & Sto trascurando una diagnosi $d a$ non perdere? \\
\hline
\end{tabular}


cognitivi (ad esempio, influenze di contesto, parzialità di inquadramento) $\mathrm{o}$ in caso di difficile coinvolgimento del paziente, deficit di conoscenze, pressione temporali, presentazioni ad alto rischio; una rivalutazione della diagnosi è invece necessaria, se l'evoluzione clinica o la risposta al trattamento sono diverse dall'atteso, il paziente peggiora, si è in prossimità del cambio turno o della dimissione 0 in caso il paziente/caregiver esprimano preoccupazioni o perplessità. Le strategie per la rivalutazione della diagnosi possono essere individuali, come il Timeout diagnostico, di gruppo come la sfida dei team Rosso e Blu; basate su servizi specialistici o sul ricorso a medici più esperti. L'approccio Take 2 - think, do aiuta a escludere lo scenario peggiore, a identificare presentazioni atipiche o rare, rivalutare $i$ pazienti che non migliorano, riconoscere le preoccupazioni dei pazienti e degli operatori sanitari, riconoscere i gruppi di pazienti ad alto rischio, favorire la discussione, il ricorso ad altri e l'escalation in caso di dilemmi diagnostici, favorire la comunicazione efficace in caso di trasferimento di cure.

Infine, gli aspiranti medici dovrebbero essere addestrati a un ragionamento clinico appropriato ed efficace. I dodici consigli per insegnare a evitare errori diagnostici e i dieci comandamenti per ridurre gli errori cognitivi possono essere utili a questo scopo (Tabelle 3 e 4$).{ }^{16}$

\section{Bibliografia}

1. Scott IA. Errors in clinical reasoning: causes and remedial strategies. BMJ. 2009;338:b1860.

2. Committee on Diagnostic Error in Health Care; Board on Health Care Services; Institute of Medicine; The National Academies of Sciences E, and Medicine. Overview of Diagnostic Error in Health Care. 2015.

3. Wilson RM, Harrison BT, Gibberd RW, Hamilton JD. An analysis of the causes of adverse events from the Quality in Australian Health Care Study. Med J Aust. 1999;170(9):411-5.

Tabella 3. Dodici consigli per insegnare come evitare gli errori diagnostici.

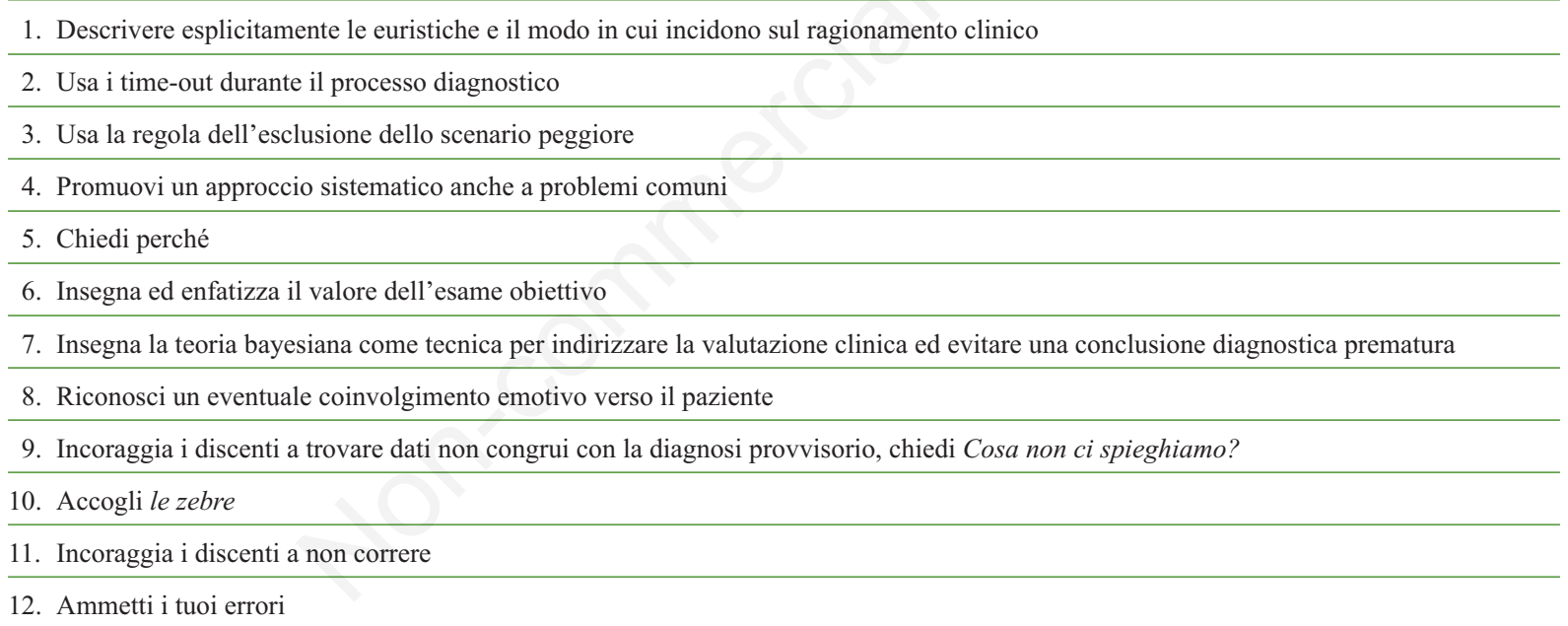

\section{Tabella 4. Dieci comandamenti per ridurre gli errori cognitivi.}

1. Rifletti su come pensi e decidi

2. Non fare affidamento sulla tua memoria quando prendi decisioni critiche

3. Renderai il tuo ambiente di lavoro facile da usare usando le informazioni dall'ultima tecnologia wireless come Tablet PC e PDA

4. Considererai altre possibilità anche se sei sicuro della tua prima diagnosi

5. Conoscerai la probabilità bayesiana e l'epidemiologia delle malattie nella tua diagnosi differenziale.

6. Sarai mentalmente a provare le condizioni comuni e gravi che hai aspettato di vedere nella tua specialità

7. Ti chiederai se sei la persona giusta per prendere la decisione finale o è necessario uno specialista dopo aver considerato i valori e $\mathrm{i}$ desideri del paziente

8. Prenditi tempo per decidere e non farti pressare da nessuno

9. Creati procedure affidabili e rivedi le decisioni prese a distanza

10. Registra in un database i problemi del tuo paziente e le decisioni prese, per la rivalutazione e il miglioramento 
4. Schiff GD, Hasan O, Kim S, Abrams R, Cosby K, Lambert BL et al. Diagnostic error in medicine: analysis of 583 physician-reported errors. Arch Intern Med. 2009;169(20):1881-7.

5. Ely JW, Levinson W, Elder NC, Mainous AG $3^{\text {rd }}$, Vinson DC. Perceived causes of family physicians' errors. J Fam Pract. 1995;40(4):337-44.

6. Podbregar M, Voga G, Krivec B, Skale R, Pareznik R, Gabrscek L. Should we confirm our clinical diagnostic certainty by autopsies? Intensive Care Med. 2001;27(11):1750-5.

7. Graber ML, Franklin N, Gordon R. Diagnostic error in internal medicine. Arch Intern Med. 2005;165(13): 1493-9.

8. Phua DH, Tan NC. Cognitive aspect of diagnostic errors. Ann Acad Med Singapore. 2013;42(1):33-41.

9. Clinical Excellence Commission. Diagnostic Error: Learning Resource for Clinicians. 2015. http://www. cec.health.nsw.gov.au/_data/assets/pdf_file/0005/3058 43/6.Diagnostic-Error-Learning-resource-for-clinicians. pdf. Last accessed October $25^{\text {th }} 2019$.

10. Health Research \& Educational Trust. Improving Diagnosis in Medicine Change Package. 2018. http:// www.hret-hiin.org/. Last accessed October $25^{\text {th }} 2019$.

11. Commission CE. Diagnostic Error: Learning Resource for Clinicians. http://www.cec.health.nsw.gov.au/ data/assets/pdf_file/0005/305843/6.Diagnostic-ErrorLearning-resource-for-clinicians.pdf. 2015. Accessed October $25^{\text {th }} 2019$.

12. Graber ML. Educational strategies to reduce diagnostic error: can you teach this stuff? Adv Health SciEduc Theory Pract. 2009;14 Suppl 1:63-9.

13. Riches N, Panagioti M, Alam R, Cheraghi-Sohi S, Campbell S, Esmail A et al. The Effectiveness of Electronic Differential Diagnoses (DDX) Generators: A Systematic Review and Meta-Analysis. PLoS One. 2016;11(3):e0148991.

14. Croskerry P, Singhal G, Mamede S. Cognitive debiasing 1: origins of bias and theory of debiasing. BMJ QualSaf. 2013;22 Suppl 2:ii58-ii64.

15. Clinical Excellence Commission. Take 2 - think, do Project. 2015. http://www.cec.health.nsw.gov.au/ data/ assets/pdf_file/0008/305846/3.Take-2-Think-DoInformation-for-Clinicians.pdf. Last accessed October $28^{\text {th }} 2019$.

16. Graber ML, Kissam S, Payne VL, Meyer AN, Sorensen A, Lenfestey $\mathrm{N}$ et al. Cognitive interventions to reduce diagnostic error: a narrative review. BMJ QualSaf. 2012;21(7):535-57. 


\title{
Metodologia clinica e EBM: vantaggi e limiti nel mondo reale dei pazienti complessi - Il mondo reale
}

\author{
Tiziana Marcella Attardo \\ UO MCAU, Ospedale Barone Lombardo di Canicattì (AG), ASP Ag, Italia
}

\section{Introduzione}

La Evidence Based Medicine (EBM) - nella originale definizione di David L. Sackett ${ }^{1}$ - costituisce un approccio alla pratica clinica in cui le decisioni risultano dall'integrazione tra l'esperienza del medico e l'utilizzo coscienzioso, esplicito e giudizioso delle migliori evidenze scientifiche disponibili, mediate dalle preferenze del paziente. ${ }^{2}$

Pertanto una corretta diagnosi e un conseguente trattamento appropriato devono essere frutto della simbiosi tra una significativa esperienza clinica (casistica clinica numerosa e variegata) e un'ottima applicazione dei risultati degli studi clinici, prove di una letteratura medica qualificata. ${ }^{2}$

Inoltre, in uno storico editoriale di Sackett et al., gli autori introducono come componenti dell'EBM, accanto alle evidenze dalla ricerca clinica e la expertise individuale del medico, i diritti e le preferenze dei pazienti (Figura 1). ${ }^{3}$

\section{Ruolo e limiti dell'EBM}

La Evidence-based Medicine costituisce, indubbiamente, un importante modo per garantire che l'atto medico - sia esso diagnostico, terapeutico o palliativistico - si basi sui risultati di ampie sperimentazioni di rilievo scientifico. Certamente, senza expertise clinica, la pratica rischia di essere tirannizzata dalle evidenze, perché anche eccellenti evidenze possono essere inapplicabili o inappropriate

Corrispondente: Tiziana Marcella Attardo, UO MCAU, Ospedale Barone Lombardo, Canicattì (AG), ASP Ag, Italia. E-mail: claudia.fantucchio@alice.it

Articolo pubblicato secondo la Creative Commons Attribution NonCommercial 4.0 License (CC BY-NC 4.0).

${ }^{\circ}$ Copyright: the Author(s), 2020

Licensee PAGEPress, Italy

QUADERNI - Italian Journal of Medicine 2020; 8(1):37-45 per il paziente individuale; così come, senza le migliori evidenze aggiornate (current) la pratica rischia di diventare rapidamente obsoleta, non contestualizzata, con rischi di malpractice sui pazienti. ${ }^{1,4}$

Nella messa in pratica tre sono le componenti dell'EBM, che richiedono attitudini, strumenti e competenze da parte dei clinici, non ancora inserite stabilmente nei curricula formativi e professionali. ${ }^{5}$ La prima, la componente essenziale, richiede al medico di avvertire bisogno d'informazione al fine di soddisfare il divario di conoscenza emerso dall'incontro con il paziente e di convertirlo in quesiti clinico-assistenziali ben definiti. La seconda, la componente tecnica, gli consente di ritrovare con la massima efficienza le migliori evidenze disponibili in letteratura e di interpretarle criticamente nella loro validità interna ed applicabilità clinica. Infine la terza, il clinical judgment determina il peso decisionale delle evidenze, tenendo conto sia delle preferenze aspettative del paziente, sia del contesto sociale, organizzativo ed economico. ${ }^{5}$

Attualmente tutti i clinici e tutte le società scientifiche di Medicina riconoscono ed accettano il ruolo della EBM come strumento dell'attività clinica. Vi sono tuttavia anche limitazioni oggettive: intanto le aree, come le malattie rare, poco interessate dalle ricerche. Inoltre si evidenziano rilevanti difetti di trasferibilità delle linee - guida, generate dalle migliori evidenze scientifiche, a causa di limiti di età, sesso, etnie dei pazienti e contesti assistenziali, oltre che alla coesistenza di più patologie concomitanti, comuni specie tra i soggetti anziani. La valutazione critica delle ricerche, specie in ambito terapeutico, determina inoltre non di rado notevoli differenze di interpretazione circa i limiti, l'attendibilità, la rilevanza, la significatività clinica e la trasferibilità che possono essere a esse riconosciute, lasciando inevitabilmente margini importanti di soggettività nel giudizio finale dei singoli clinici, dei responsabili dei servizi sanitari e degli stessi pazienti. ${ }^{6}$

Infatti il clinico non può attingere all'EBM come ad un tram con un percorso predefinito, obbligato a seguire un determinato binario per portarlo a destinazione; deve, invece, rivolgersi all'EBM come 
un'automobile con cui sia possibile, in certi casi, scegliere una strada alternativa - per diverse ragioni rispetto a quella indicata dalla mappa ufficiale. ${ }^{7}$

Gli studi inclusi nelle revisioni sistematiche in base ai quali vengono costruite le linee guida vengono effettuati su pazienti selezionati. Certamente gli studi clinici randomizzati e controllati (RCT) rappresentano il disegno di studio più affidabile per acquisire evidenze sperimentali sull'efficacia degli interventi sanitari (in particolare terapeutici), ma esistono numerosi problemi per adattare i loro risultati al paziente individuale. Essi vengono generalmente condotti su popolazioni selezionate ed omogenee - e come tali spesso molto diversi da quelli della pratica corrente e del mondo reale - escludendo i pazienti complessi (comorbidità, anziani), le donne, i bambini, che rischiano di compromettere la validità interna dello studio. ${ }^{8}$

I trials, infatti, reclutano i casi da studiare secondo rigidi criteri che escludono alcuni pazienti per età, comorbosità, inaffidabilità nel seguire le cure, etc.; i criteri di esclusione dagli studi riguardano, però, soggetti che, abitualmente, si incontrano nella pratica corrente. ${ }^{9}$

Da qui la domanda è se le linee - guida derivate dalle migliori evidenze scientifiche possano essere applicate - e se si, con quali limiti - nel mondo reale, ai pazienti internistici, sempre più complessi, multipatologici, comorbidi, spesso fragili per età e per condizioni cliniche, tanto da non potere essere inclusi negli RCT, di fatto disegnati su pazienti ideali, fuori dal mondo reale.

Inoltre la competenza e la motivazione di chi

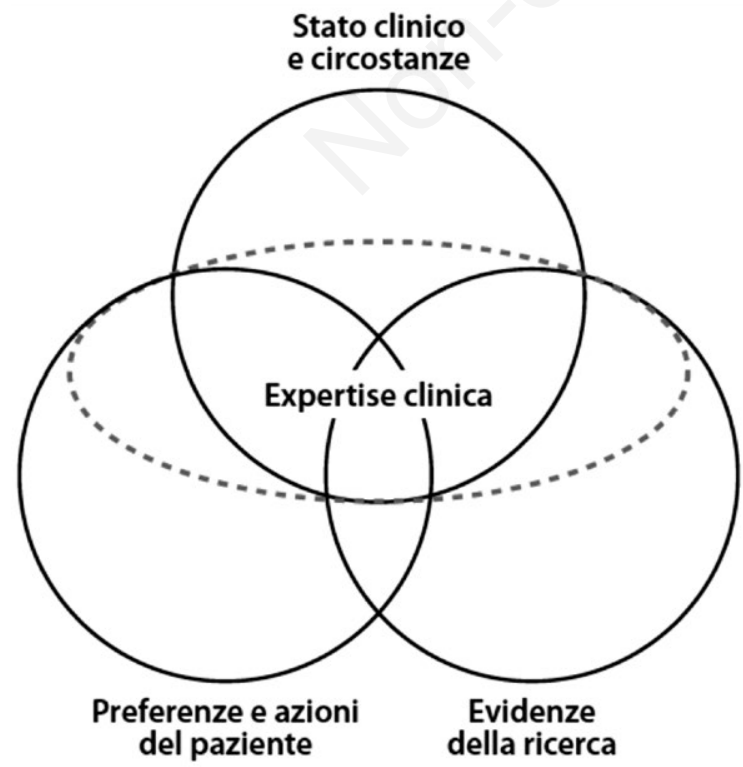

Figura 1. Componenti dell'EBM. Tradotta ed adattata da Haynes, 2002. ${ }^{3}$ partecipa ad un RCT - oltre che le condizioni organizzativo-assistenziali - sono ideali rispetto alla realtà. La pratica clinica quotidiana è fatta invece di pazienti reali, assistiti da medici reali che operano in strutture sanitarie reali, per cui spesso è difficile riconoscere tra la popolazione selezionata di un RCT la variabile e complessa (comorbidità, polifarmacia) individualità del singolo paziente. ${ }^{8}$

Inoltre, i risultati di un RCT sono medi e, quindi, non possono prevedere la risposta individuale del singolo paziente. In aggiunta gli end-point misurati dagli RCT - spesso surrogati invece che clinicamente significativi - sia i metodi utilizzati per riportare i risultati - quasi sempre misure relative che hanno la capacità di enfatizzare l'efficacia dei trattamenti rischiano di effettuare alcune distorsioni percettive che possono condizionare le scelte del clinico, penalizzando la risposta clinica del paziente. ${ }^{8}$

\section{L'EBM e le aree grigie}

In molti settori della medicina non esiste ricerca di buona qualità, ma numerose ed ampie zone grigie dove esiste incertezza sull'efficacia di un intervento diagnostico-terapeutico e/o delle sue alternative. Questo, però, non dovrebbe paralizzare la flowchart decisionale, perché la EBM richiede di fondare le decisioni cliniche sulle migliori evidenze disponibili e non sulle migliori evidenze possibili che in alcune aree della medicina - per motivi etici, economici o metodologici - potrebbero non essere mai disponibili. ${ }^{8}$

La carenza di RCT e di studi clinici di valore scientifico per numerosità di pazienti arruolati e/o per rigore metodologico in ambito di aree grigie, di malattie rare e/o di pazienti complessi pone la problematica della carenza di evidenze di qualità in questi ambiti e di produzione di Linee guida. In realtà il secondo editoriale di Sackett ${ }^{10}$ demitizza l'idea che esistano disegni di studio superiori e afferma il concetto che $\mathrm{i}$ differenti problemi di medicina devono essere affrontati con differenti disegni di studio; se l'efficacia di un nuovo trattamento esige il disegno sperimentale del trial controllato e randomizzato, gli effetti del trattamento nella pratica corrente richiedono studi osservazionali siano essi definiti studi di coorte, di outcome o di effectiveness. ${ }^{4}$

\section{Reale impatto dell'EBM nella pratica clinica quotidiana ed ostacoli alla sua implementazione}

Indubbiamente l'EBM rappresenta una strategia efficace per migliorare la qualità delle cure.

La conoscenza dei principi e dei metodi della medicina basata sulle prove di efficacia (EBM) e il 
riferimento alle linee guida per la pratica clinica sono considerati strumenti chiave per migliorare la qualità delle cure.

In realtà l'EBM viene applicata molto meno di quanto non sarebbe desumibile da quanto affermato finora e, fin dall'inizio della sua introduzione, si sono osservate numerose barriere alla sua applicazione. Ciò dipende, in parte, dalle intrinseche difficoltà nel reperimento e nella valutazione degli studi scientifici e nella loro trasferibilità al caso clinico concreto, ma soprattutto dal persistere di abitudini professionali consolidate dalla routine (specie nell'uso dei test diagnostici e nelle decisioni terapeutiche). Molteplici appaiono le barriere per l'implementazione dell'uso dell'EBM tra cui il deficit di risorse, la mancanza di tempo, la carenza di competenze e di conoscenze adeguate, l'accesso inadeguato e queste sono stati riscontrati nelle cure primarie, nelle cure ospedaliere/specialistiche, nelle cure di riabilitazione, nell'educazione medica, nella gestione e nel processo decisionale. Certamente l'identificazione di esse è il primo step per poterle superare. ${ }^{11}$

La legislazione italiana ${ }^{12}$ ha rivisto l'impianto giuridico che norma le responsabilità dei professionisti sanitari e la sicurezza delle cure, prescrivendo il rispetto delle linee guida e delle buone pratiche. Ma quanto è presente e quanta consapevolezza c'è da parte degli operatori sanitari? Quanta l'attitudine e le conoscenze degli operatori sanitari nei confronti di EBM e linee guida? Allarmanti appaiono i dati di una recente indagine presso l'ASL5 La Spezia dove è stato effettuato un censimento di tutti i documenti clinici archiviati come linee guida e protocolli clinici presso la Direzione Generale fino al 30 maggio 2016. La valutazione dei documenti è stata condotta attraverso la Scheda di ricognizione dell'attività di produzione e revisione dei PDTA elaborata dalla Regione Liguria. L'attitudine e le conoscenze degli operatori sanitari sono state valutate attraverso interviste semistrutturate. A fronte delle iniziative del legislatore sulla responsabilità civile e sicurezza delle cure che accrescono l'importanza delle linee guida, la ricerca ha evidenziato una realtà nel complesso digiuna dei concetti fondamentali dell'EBM e dei metodi di produzione ed uso delle linee guida stesse. Anche se l'attitudine è genericamente positiva, le barriere all'uso sembrano essere predominanti, vissute più come ostacoli che come stimolo. In contesti periferici o in ospedali di dimensioni medio/piccole, le linee guida potrebbero rimanere confinate a un ruolo giuridico, con scarse ricadute a livello professionale. ${ }^{13}$

\section{La medicina della complessità}

Se andiamo a ricercare la parola complessità sul dizionario Treccani, ${ }^{6}$ il termine viene definito come caratteristica di un sistema (perciò definito complesso), concepito come un aggregato organico e strutturato di parti tra loro interagenti, in base alla quale il comportamento globale del sistema non è immediatamente riconducibile a quello dei singoli costituenti, dipendendo dal modo in cui essi interagiscono. ${ }^{6}$

La comune origine $^{14}$ filologica dei termini complesso e complicato risiede nella radice indoeuropea: plek (piega, intreccio). Infatti il termine complicato deriva da cum + plicare e cioè con pieghe, più pieghe, quindi nell'ambito medico, si riferisce a più malattie. Al contrario il termine complesso, derivando dal latino cum + plectere (con intrecci), sta ad indicare la difficoltà a sciogliere l'intreccio e, nell'ambito medico, indica come le diverse patologie compresenti nello stesso paziente tentino ad interagire tra di loro - intrecciandosi per l'appunto - rendendo difficile ed impegnativo governarle e valutarle.

Queste premesse bene descrivono la difficoltà e l'impegno che nel mondo reale comporta la presa in carico di questi pazienti complessi. Il miglioramento delle condizioni sociosanitarie, l'aumento della sopravvivenza a condizioni cliniche un tempo fatali e l'invecchiamento della popolazione hanno portato progressivamente a una profonda modificazione dello scenario di cura, con un progressivo incremento delle malattie ad andamento cronico, spesso presenti contemporaneamente nello stesso individuo. Oggi l'obiettivo principale è rappresentato dalla gestione del malato cronico e dalla definizione di nuovi percorsi assistenziali, o in grado di prendere in carico l'individuo nel lungo termine e prevenire la disabilità, garantendo, quindi, sia la continuità assistenziale ospedale-territorio, sia l'integrazione degli interventi sociosanitari. ${ }^{14}$

La presenza di più patologie croniche induce il malato a richiedere l'intervento di più figure professionali specialistiche, con il rischio di interventi frammentati, focalizzati più sul trattamento della singola malattia che sulla gestione del malato nella sua interezza, o di istruzioni diagnostico-terapeutiche contrastanti, che rendono difficoltosa la partecipazione del paziente stesso al processo di cura, di primaria importanza nelle patologie croniche, contribuendo, in aggiunta, all'aumento della spesa sanitaria. I pazienti affetti da più patologie croniche hanno un maggiore rischio di andare incontro ad outcome negativi, quali aumento della morbidità, sia fisica sia psicologica, aumentata frequenza e durata di ospedalizzazione, aumentato rischio di disabilità e non autosufficienza, peggiore qualità di vita e aumento del rischio di mortalità. Per il processo di cura e di assistenza di questi pazienti è necessario acquisire le conoscenze e diventare padroni di strumenti atti ad identificare, 
nell'ambito di una visione globale dell'individuo malato, fra tutti i determinanti e le loro connessioni, quelli che hanno un ruolo chiave nell'influenzare lo stato di salute dell'individuo, in modo da porre delle priorità e pianificare una strategia terapeutica e assistenziale multiprofessionale e personalizzata. In tal modo, grazie a nuove modalità assistenziali, si potrebbero fornire ai malati cronici e complessi nuovi percorsi di cura, sempre più individualizzati ed in grado di rispondere ai bisogni clinici. Questo favorirebbe la presa in carico a lungo termine, garantirebbe la continuità assistenziale ospedaleterritorio, l'integrazione degli interventi sociosanitari, favorendo il loro mantenimento, il più a lungo possibile, nel proprio ambiente di vita, migliorando la qualità di vita del paziente e l'umanizzazione del trattamento. ${ }^{15}$

\section{L'EBM e il paziente complesso}

La complessità in Medicina considera l'insieme delle diverse condizioni morbose non solo in quanto compresenti, ma nella loro interazione multidimensionale (comorbidità, multimorbidità a genesi comune o diversa, convergenza su elementi clinici comuni ed interconnessione con acuzie e cronicità e con l'intensità di cura necessaria). ${ }^{14}$

La complessità non ha ancora un adeguato inserimento nell'insegnamento clinico, essenzialmente basato sulle ontologie. Quest'ultimo, infatti, con approccio riduzionistico, ha l'obiettivo di trasferire puntualmente ma separatamente le conoscenze e le competenze generate attraverso l'articolazione della medicina della specialità.

L'approccio alla complessità, invece, richiede la capacità di includere tutti i diversi elementi rilevanti presenti, poiché tutti contribuiscono alla genesi del quadro clinico rendendo essenziali per la formazione, insieme all'EBM, l'insegnamento per problemi e la comunicazione per affrontare la dimensione persona del paziente nella sua globalità. La presa in carico di questi pazienti complessi richiede certamente una valutazione di tipo tecnico - clinico affinché lo studio del paziente sia adeguato, ma anche una di tipo umanistico - comunicativo per una appropriata analisi del paziente, e di tipo economico - gestionale secondo i criteri dell'Health Technology Assessment (HTA), ${ }^{14}$ processo multidisciplinare che, grazie alle informazioni sulle questioni cliniche, economiche, sociali ed etiche connesse all'uso di una tecnologia sanitaria, in modo sistematico, trasparente, imparziale e solido, ne convalida l'utilizzo.

Non esiste una definizione univoca di complessità: in Medicina Interna studiare la complessità significa comprendere il bisogno di ricomposizione, di riaggregazione delle conoscenze, superare la frammentarietà. ${ }^{2}$ La complessità rende la Medicina una scienza probabilistica, con un elevato rischio di errore, per l'incertezza che permea le decisioni mediche, che pur devono essere adottate in un tempo limitato e in un contesto di conoscenza non sempre definito. ${ }^{2}$

La scomposizione del malato nelle singole patologie da cui è afflitto porta lo specialista d'organo a un proprio percorso diagnostico e terapeutico che spesso si sovrappone o addirittura confligge con quello di altri colleghi, portando a iperprescrizioni di indagini laboratoristiche e strumentali e a politerapie farmacologiche contrastanti e dannose. In quest'ottica si inserisce la possibilità di intervenire in modo incisivo, con provvedimenti ad hoc, anche sui costi spropositati della medicina difensiva, partendo dai presupposti fondanti della EBM ma, anche superandoli, non essendo questi in grado di essere rigidamente e funzionalmente applicabili al paziente complesso. In quest'ottica di approccio integrato al paziente va sicuramente incentivata la ricerca in merito ai principi ispiratori della systems medicine ovvero su approcci olistici integrati multidisciplinari (scienze biologiche e mediche in senso ampio - quindi comprendenti l'analisi approfondita delle componenti socioeconomiche e psicologiche - intorno alla persona, medical informatics e computer science, fino alla modellizzazione matematica per l'ottimizzazione della sperimentazione clinica) che affrontino il paziente complesso con strumenti di analisi potenzialmente capaci di comprendere gli elementi strutturanti la complessità e quindi di suggerire strategie di ottimizzazione terapeutica e di gestione. ${ }^{14}$ Indubbiamente alla ricerca biomedicale in senso stretto, con le caratteristiche di olisticità descritte, deve poi affiancarsi un approccio severo all'analisi degli aspetti di sostenibilità gestionale finalizzato all'ottimizzazione dell'impiego delle risorse, sempre più limitate, per traslare best practices veramente innovative ed efficienti. ${ }^{14}$

La malattia-ontologia non corrisponde a un fenotipo unico e stabile, ma diverso per ogni individuo, a cui deve essere adattato ogni intervento clinico. I sistemi biologici del nostro corpo costituiscono una realtà unitaria, che supera le singole parti, per cui la comprensione del sistema richiede la valutazione contemporanea delle sue componenti, senza regole gerarchiche.

La medicina della complessità si esercita con la sintesi, che diviene sinergia, tra l'EBM e la medicina narrativa, cogliendo attraverso tutti i sensi ogni dinamica della vita dell'individuo. ${ }^{14}$

La metodologia è quella abituale della buona medicina, accentando il rigore metodologico con cui si svolgono: anamnesi ed esame obiettivo; individuazione del sintomo/sintomi guida (ovvero motivo/i del ricorso alle cure); impostazione dell'iter diagnostico con approccio inclusivo; impostazione 
dell'iter terapeutico integrato; impostazione di un programma di follow-up e di proseguimento corretto del percorso clinico. Ciascuna di queste fasi si deve svolgere con approccio inclusivo, senza gerarchie esclusive, ma includendo e valorizzando ogni elemento con approccio olistico e sistematico.

\section{Il Fenoma Complesso}

La odierna realtà con cui il medico si trova attualmente ad interagire e che lo porta ad operare su pazienti affetti da più condizioni morbose coesistenti impone di guardare oltre le definizioni tassonomiche tradizionali e di individuare nuove presentazioni fenotipiche relative ai pazienti, siano essi adulti ma anche, e soprattutto, anziani. I progressi della medicina hanno infatti permesso, da un lato, di curare, ma non di guarire, patologie che un tempo avevano un esito letale precoce (es. cardiopatie congenite, difetti genici) e, dall'altro, hanno prolungato la sopravvivenza di pazienti affetti da malattie croniche (es. cardiovascolari, metaboliche). Tutto ciò ha condotto all'emergenza di quadri clinici/fenotipi complessi; il fenotipo o Fenoma Complesso, lungi dall'essere una semplice sommatoria delle condizioni morbose che lo compongono, rappresenta un'entità fenomenica con caratteristiche peculiari in termini di eziopatogenesi, necessità terapeutiche e prognosi. La gestione del paziente complesso richiede un approccio che vada al di là del semplice coordinamento delle varie prestazioni specialistiche, ma che si configuri come messa a punto di percorsi diagnostico-terapeutici-riabilitativi il più possibile individualizzati, con buon rapporto costo/efficacia, e che prevedano sempre di più l'empowerment del paziente e della sua famiglia e la costituzione di percorsi assistenziali in continuità ospedale-territorio. ${ }^{14}$

\section{Revisione critica dei modelli gestionali}

La realizzazione di un modello di assistenza sanitaria che possa prendere in carico in modo sostenibile i pazienti con patologie croniche richiede una nuova definizione dei modelli gestionali. In tale ottica lo sviluppo di una sanità d'iniziativa, ovvero di un modello assistenziale che sia in grado di intervenire, quando possibile, prima dell'insorgere della malattia e di gestire la malattia stessa in modo tale da rallentarne il decorso e limitarne le riacutizzazioni, garantisce al paziente interventi adeguati e differenziati in rapporto al livello di rischio.

Il Chronic Related Group (CReG) della Regione Lombardia ne rappresenta un esempio. Esso classifica gli assistiti in funzione della cronicità individuando, con una particolare tecnica di clusterizzazione, le prestazioni più probabili e ridefinisce, di conseguenza, un Piano di Assistenza Individuale. Il CReG fotografa l'assistito nella sua condizione reale di bisogno con l'obiettivo di farsene carico nel modo più esaustivo possibile, organizzando un modello assistenziale che supporti il paziente e incrementi la sua compliance con le cure di cui ha bisogno. ${ }^{14}$

Altro modello è l'Expanded Chronic Care Model (Expanded CCM) della Regione Toscana, capace di superare l'approccio per patologia e focalizzare l'attenzione sul rischio, classificando i pazienti in base al rischio di cronicità. Esso espande l'attenzione non solo alle condizioni sanitarie, ma anche a quelle sociali, economiche e culturali degli assistiti e alla prevenzione primaria. ${ }^{14}$

\section{Gli strumenti tecnologici degli approcci di sistema alla complessità: systems biology e systems medicine}

Lo sviluppo tecnologico delle metodiche di biologia molecolare e cellulare consente l'analisi su vasta scala di DNA, mRNA, proteine e metaboliti contribuendo al progresso della systems biology. La systems biology, grazie a una rapida evoluzione della computer science applicata alla biologia e alla medicina, ha la capacità di prevedere l'outcome di un certo stato di modifiche nell'assetto geneticoproteomico-metabolomico-esposomico e quindi di consentire lo sviluppo di nuove strategie diagnosticoterapeutiche. Gli approcci di systems biology contribuiranno alla raccolta di informazioni rilevanti per l'identificazione di nuovi target terapeutici e nuovi approcci innovativi di diagnosi e terapia che consentiranno, a loro volta, l'applicazione di un approccio personalizzato di systems medicine. ${ }^{14}$

\section{Ipotesi per una gestione sostenibile della complessità}

Una gestione scientificamente accettabile, organizzativamente praticabile ed economicamente sostenibile, del paziente complesso richiede un approccio alla sua gestione attraverso un nuovo modello di assistenza sanitaria, che faccia della medicina di sistema o systems medicine la sua base concettuale, che colloca realmente i singoli cittadini al centro del processo dell'assistenza sanitaria, conciliando le differenze individuali in tutte le fasi del processo, dalla prevenzione, dalla diagnosi, dal trattamento al follow-up. ${ }^{14,16}$

Il primo passo verso tale sistema è conoscitivo: stanti le attuali difficoltà dei sistemi informativi sanitari, si è posta la necessità di identificare la popolazione dei malati complessi, con le relative 
caratteristiche, anche allo scopo di delineare schemi e percorsi gestionali appropriati. Fondamentale è la costruzione di una piattaforma di gestione e monitoraggio di tutti i fattori che possono influenzare lo sviluppo della malattia in un dato individuo, tra cui non solo i fattori genetici e biologici, ma anche le influenze ambientali e quelle legate allo stile di vita, oltre che i singoli episodi assistenziali, proprio allo scopo di integrare informazioni complesse provenienti da molteplici fonti di dati e generare un output utilizzabile per sostenere la salute dei singoli cittadini. Solo così si può conseguire l'orientamento dei sistemi organizzativo-gestionali.

L'attuale contingenza di scarsità di risorse, con la necessità improrogabile dell'allocazione prioritaria delle risorse in maniera quanto più ottimale ed efficiente possibile, impone, proprio a partire da quest'ambito, un cambiamento dei sistemi sanitari, nel medio - lungo periodo, verso logiche di sostenibilità e di creazione di valore (rapporto costo/outcome) per il cittadino-paziente. È tuttavia indubbio come tale trasformazione sarà possibile agendo, oltre che sullo sviluppo organizzativo, sulla cultura e sullo sviluppo dei sistemi di gestione delle risorse, davvero integrati e orientati ai problemi di salute e non più alla sola e puntuale erogazione dei singoli servizi di cura. ${ }^{14,16}$

Tutto inizia dalla comprensione della complessità, tipica del mondo reale e completamente differente dai sistemi semplici e lineari (Tabella 1 ) ${ }^{17}$ I sistemi sanitari funzionano come sistemi adattativi complessi piuttosto che rigide, lineari o meccaniche organizzazioni. Componenti critiche dei primi sono creatività e innovazione, mentre le seconde utilizzano principalmente controllo e prevedibilità. Forse un buon equilibrio tra entrambi può dare valore alla prestazione di cure, in quanto entrambi gli approcci sono necessari per raggiungere l'eccellenza. ${ }^{17}$

L'EBM, divenuta nel 2017 EBHC (Assistenza sanitaria basata sull'evidenza) ${ }^{18}$ non riconosce più il risultato di una media campionaria di un RCT come applicabile tout court, in modo non problematico, allo scenario del singolo paziente. Esiste una comprensione più sofisticata del processo che dall'evidenza scientifica viene traslato ad una situazione particolare, al singolo caso clinico, a quel preciso paziente con la sua situazione particolare. ${ }^{18}$

Ad esempio, ispirati ai concetti di processo decisionale condiviso ${ }^{19}$ e di cura incentrata sulla persona,$^{20}$ recentemente il supporto alle decisioni cliniche individualizzate è stato pensato grazie alla fornitura di nuovi strumenti in nuovi formati, come, ad esempio, l'app Magic ${ }^{21}$ o le griglie delle opzioni. ${ }^{22}$

La capacità di effettuare ricerche online di informazioni sulla salute da parte dei pazienti - per esempio - preoccupa i clinici perché spesso i pazienti si confondono e posso avere aspettative verso trattamenti che, però. hanno scarse prove scientifiche a sostegno. Allo stesso tempo, la scienza della sintesi delle evidenze della ricerca è avanzata al punto in cui è sempre più possibile quantificare i compromessi terapeutici e descrivere l'equilibrio tra danni e benefici per i singoli pazienti. Per questo, nel tentativo di personalizzare l'uso delle prove e garantire che le scelte siano coerenti con le loro preferenze personali dei singoli pazienti, sono stati sviluppati strumenti per i pazienti che traducono le prove esistenti e che aiutino i pazienti e i medici a lavorare insieme per decidere i passi successivi. Naturalmente in primo luogo è evidente che il processo decisionale in ambito sanitario richiede collaborazione e confronto e, in secondo luogo, per raggiungere questo obiettivo è necessario avere strumenti progettati per personalizzare l'assistenza a livello individuale. ${ }^{18}$

La novità di questi strumenti è che non prescrivono solo la natura oggettiva (funziona il farmaco?), ma presentano anche argomenti tratti dalla cultura umana (i pazienti lo apprezzano? È conveniente?). ${ }^{18}$

Tali strumenti includono i pazienti nel processo decisionale e si allontano dall'idea che la medicina debba essere determinata esclusivamente da ciò che è meglio dal punto di vista medico, consentendo di considerare anche le priorità, le preoccupazioni e le

Tabella 1. Confronto delle caratteristiche tra un sistema complesso ed uno tradizionale.

\begin{tabular}{ll}
\hline SISTEMI COMPLESSI & SISTEMI TRADIZIONALI \\
\hline Sono organismi viventi & Sono macchine \\
\hline Sono imprevedibili & Sono a comportamento controllato \\
\hline Sono creativi & Sono controllabili e prevedibili \\
\hline Abbracciano ed incorporano la complessità & Sono rigidi, autoconservativi \\
\hline Evolvono continuamente & Trovano comfort nel controllo \\
\hline
\end{tabular}

Tradotta ed adattata da Freddi e Romàn-Pumar, 2001. ${ }^{17}$ 
preferenze dei pazienti. ${ }^{23}$ Un esempio è il modello chiamato deliberazione collaborativa, ${ }^{23}$ costruito su 5 proposizioni (Tabelle 2 e 3 ) che suggerisce alla pratica clinica una forma di assistenza centrata sul paziente, colloquio motivazionale, definizione degli obiettivi, pianificazione dell'azione e processo decisionale condiviso.

\section{Conclusioni}

Qualche anno fa la rivista L'Espresso ha ironizzato sul gioco dell'oca ${ }^{24}$ sottolineando che nella medicina moderna sembra sparito lo sguardo d'insieme e ormai chi sta male viene spedito da mille diversi esperti per capire cosa ha. Il giornalista alla fine concludeva: ... in ogni caso forse un problema ce l'ha, la medicina contemporanea così iperspecializzata e iperfondata sulle più evolute macchine diagnostiche. E il problema è che quasi nessuno ti guarda più per intero, dai piedi alla testa, dall'anima all'unghia. Sembra che ciascuno di noi non sia più una cosa sola e totalmente interconnessa al suo interno, bensì un'addizione di componenti meccaniche, pistoni, giunti, cuscinetti a sfera, assali. Tu stai male e vai dal medico di base, che ti spedisce dallo specialista dei pistoni, il quale ti assicura il funzionamento del pistone quindi lui non c'entra e ti assegna al tecnico dei cuscinetti a sfera, proviamo un po' a vedere che dice lui, eccetera. E a questo punto al sottoscritto - rigidamente razionalista, illuminista, scientista, cartesiano e kantiano - viene tuttavia un dubbio: non è che oggi hanno tanto successo i bufalari della salute, on line o meno, perché la medicina vera sbaglia qualcosa, almeno nell'approccio al paziente?. ${ }^{24}$

Ma a seguire arrivava, puntuale, la risposta del Dr Fontanella Andrea - allora Presidente Nazionale Fadoi, ora Past President e Presidente della Fondazione FADOI - che ricordava all'illuminista e razionalista giornalista che c'è chi ogni giorno lavora con un approccio d'insieme e di sintesi. La soluzione che viene proposta - continuava il Dr Fontanella - per fare fronte a questo fenomeno sta nell'affermazione del metodo della Medicina Interna, che si rivolge al malato nella sua interezza, partendo da un attento colloquio e da un puntiglioso esame fisico, in molti casi sufficienti per porre una diagnosi valida. Gli internisti lavorano nei reparti di Medicina Interna presenti in tutti gli ospedali e seguono quasi due milioni di ricoveri l'anno. L'internista lavora come un direttore d'orchestra. Conosce la partitura, utilizza $i$ vari strumenti e li fa esprimere al meglio. É uno specialista della complessità, anche perché spesso $i$ pazienti, specialmente se anziani, hanno più malattie insieme. Oggi gli internisti in tutto il mondo

Tabella 2. Elementi della Medicina Collaborativa.

\begin{tabular}{l}
\hline Impegno interpersonale costruttivo \\
\hline Riconoscimento di azioni alternative \\
\hline Apprendimento comparativo \\
\hline Costruzione delle preferenze ed elicitazione \\
Integrazione delle preferenze \\
Tradotta ed adattata da Elwyn et al., 2014. ${ }^{23}$
\end{tabular}

Tradotta ed adattata da Elwyn et al., 2014.23

Tabella 3. Elementi della Medicina Collaborativa.

Fornire prove affidabili ed applicare nuovi standard e sistemi per la sintesi delle prove (ulteriori lavori dovrebbero concentrarsi su strategie di presentazione e diffusione ottimali e l'impegno a creare versioni rivolte al pubblico con strategie ottimali di coinvolgimento dei pazienti)

Creare strumenti generici di assistenza personalizzata: se i pazienti possono capirli, lo saranno anche i medici e c'è più possibilità che vengano generate conversazioni migliori. I clinici impegnati apprezzano gli strumenti brevi, ma saranno disposti a leggere versioni più lunghe se hanno bisogno di saperne di più sulle prove sottostanti

Semplificare la ricerca degli strumenti: utilizzare i metodi di ottimizzazione dei motori di ricerca per assicurarti che gli strumenti siano classificati in modo ottimale nei risultati

Creare fonti attendibili: le persone stanno diventando più scettiche, dobbiamo creare fonti attendibili di prove ed essere trasparenti sui limiti nella qualità delle prove

Portare strumenti all'incontro clinico: format di presentazione innovativi, basati su linee guida affidabili, progettati per l'uso negli incontri clinici potrebbero supportare la collaborazione e la decisione clinica

Tradotta ed adattata da Elwyn et al., 2015.22 
partecipano ai programmi della 'slow medicine', che vuol dire che fare di più non sempre equivale a fare meglio, e bisogna scegliere esami e cure appropriati $e$ di buona qualità, senza eccessi o inutili prescrizioni... Nelle situazioni complesse il ruolo dell'internista è fondamentale, perché ha visione olistica e capacità di sintesi. Il ricorso alla specializzazione estrema nasce anche dall'enorme sviluppo della tecnica, alle grandi possibilità delle diagnosi di laboratorio e genetiche, alle tecnologie di immagine sempre più sofisticate. Però in questa esplosione tecnologica il paziente ha bisogno di un inquadramento globale che tenga conto dei suoi veri bisogni e spesso anche della sua fragilità e della sua complessità, di fiducia e continuità assistenziale. Ieri avevamo comportamenti dettati dalla saggezza clinica ma meno conoscenza e minore supporto tecnico. Oggi abbiamo molta più conoscenza e spropositato sviluppo tecnico, ma minore saggezza nel gestirli. Se vogliamo garantirci il futuro del domani dobbiamo ritornare ai modi dell'ieri con la conoscenza e la tecnica dell'oggi. Gilioli ha ragione quando conclude con il dubbio che "i bufalari della salute abbiano successo perché la medicina vera sbaglia qualcosa, almeno nell'approccio al paziente». Infatti noi internisti - concludeva Fontanella - lavoriamo per ridare spazio ad una Medicina a misura d'uomo. A tutto vantaggio dell'uomo. ${ }^{25}$

La medicina basata sull'evidenza (EBM) non è un vecchio cappello (Tabella 4), ${ }^{17}$ un ricettario scritto da qualche arrogantello a beneficio di qualche ragioniere della Sanità per sopprimere la libertà del medico, una prassi medica obbligatoria, deterministica e totalitaria, una disciplina per controllare i costi e ignorare le preferenze dei pazienti, un limite invalicabile alla medicina personalizzata, umanistica, individuale. ${ }^{17}$ L'EBM resta, invece, un riferimento di eccellenza per indirizzare le decisioni cliniche, l'integrazione della propria con le migliori esperienze e con le preferenze dei pazienti, un modo per migliorare la pratica medica limitando la variabilità e gli errori laddove non esistano evidenze concrete, per identificare il gold standard e differenziare tra le diverse alternative disponibili. Certamente le evidenze devono essere integrate con un nuovo modo di pensare che si basa sulla complexity science. I sistemi sanitari operano come sistemi adattativi complessi piuttosto che come organizzazioni meccaniche, rigide e lineari e l'innovazione è uno dei risultati più importanti della complexity science. ${ }^{17}$

In ogni caso non vi sono dubbi sul fatto che la EBM abbia contribuito, in pochi anni e in modo sostanziale, a cambiare l'atteggiamento dei medici rispetto alle conoscenze scientifiche, alla natura e validità delle prove, e al modo in cui vengono prodotte e utilizzate. ${ }^{6}$

Certamente, forti dell'esperienza del'EBM, è tempo di concentrare la nostra energia su strumenti che possano aiutare a facilitare l'assistenza personalizzata dei singoli individui, ${ }^{18}$ dove l'empowerment del paziente e la sua corretta formazione/informazione rivestono un ruolo fondamentale per assicurare trasparenza, condivisione e partecipazione attiva, sia del paziente che del Clinico, al percorso terapeutico così da raggiungere quell'alleanza strategica che permetta di assicurare la scelta delle migliori opzioni, nell'ottica di una medicina sempre più efficace e personalizzata.

\section{Bibliografia}

1. Sackett DL, Rosenberg WM, Gray JA, et al . Evidence based medicine: what it is and what it isn't. BMJ 1996; 312:71-2.

2. Nardi R, Fabbri T, Belmonte G et al. Medicina interna, paziente complesso, evidence based medicine e le non

Tabella 4. L'EBM: cosa è e cosa non è!

\begin{tabular}{ll}
\hline Cosa non è & Cos $\boldsymbol{e}$ ̀े \\
\hline $\begin{array}{l}\text { Un vecchio cappello (Médecine d'Observation francese di inizio } \\
\text { del XIX secolo) }\end{array}$ & Un riferimento di eccellenza per guidare le decisioni cliniche \\
\hline $\begin{array}{l}\text { Una medicina ricettario perpetrata da qualche arrogantello per ridurre } \\
\text { i costi e per sopprimere la libertà clinica }\end{array}$ & 'integrazione della propria esperienza con quella degli altri \\
\hline $\begin{array}{l}\text { Un blocco / divieto / barriera per non fare qualcosa quando non } \\
\text { esistono prove }\end{array}$ & $\begin{array}{l}\text { Un modo per migliorare la pratica medica e limitare la variabilità e gli } \\
\text { errori creati quando non ci sono evidenze }\end{array}$ \\
\hline $\begin{array}{l}\text { Una pratica obbligatoria, deterministica, totalitaria di medicine } \\
\text { alternative disponibili }\end{array}$ & Un modo per identificare il gold standard e differenziarlo tra le diverse \\
\hline Un modo per controllare i costi e ignorare le preferenze del paziente & Un approccio valido per gli interventi medici \\
\hline $\begin{array}{l}\text { Uno strumento politico / economico / commerciale } \\
\text { Un limite alla medicina personalizzata /individualizzata }\end{array}$ & L'integrazione della decisione clinica con le preferenze dei pazienti \\
\hline Tradotta ed adattata Freddi e Romàn-Pumar, $2001 .{ }^{17}$ &
\end{tabular}


evidenze. Italian Journal of Medicine 2009; 3: 191-200.

3. Haynes RB, Devereaux PJ, Guyatt CH. Clinical expertise in the era of evidence-based medicine and patient choice. ACP Journal Club 2002; 136: A11-4.

4. Pagliaro L, Colli A. Evidence-based medicine: nuovo paradigma della medicina e marchio di garanzia nel supermercato della letteratura medica. Recenti Prog Med 2014; 105: 370-373.

5. Cartabellotta A. La medicina basata sulle evidenze: criticità e prospettive. Rec Progr Med 2006;97:640-6.

6. Enciclopedia Treccani online. Data consultazione online 18/01/2020.

7. Antiseri D. Questioni di metodologia clinica: domande a Vito Cagli. Working Papers n. 106, 2007. Pubblicazioni a cura del Centro di metodologia delle scienze sociali, LUISS Guido Carli, Roma.

8. https://www.gimbe.org/eb/limiti.html\#Areegrigie data consultazione online 16/01/2020

9. R. Festa. Principio di evidenza totale, decisioni cliniche ed Evidence Based Medicine, in Aa.Vv., Forme della razionalità medica, a cura di G. Federspil e P. Giaretta, Rubbettino, Soveria Mannelli, 2004, pp. 47-82.

10. Sackett DL, Wennberg JE. Choosing the best research design for each question. It's time to stop squabbling over the "best" methods. BMJ 1997; 315: 1636.

11. Sadeghi-Bazargani H, Tabrizi JS, Azami-Aghdash S. Barriers to evidence-based medicine: a systematic review. J Eval Clin Pract. 2014;20(6):793-802.

12. GAZZETTA UFFICIALE DELLA REPUBBLICA ITALIANA Serie generale - n. 66 MINISTERO DELLA SALUTE DECRETO 27 febbraio 2018 Istituzione del Sistema Nazionale Linee Guida (SNLG).

13. Minozzi S, Ruggiero F, Capobussi M et al. EBM, guidelines, protocols: knowledge, attitudes and utilization in the era of law on professional responsibility and safety of health care. Recenti Prog Med. 2018;109(5):294-306.

14. Quaderni n. 23 del Ministero della Salute. Criteri di appropriatezza clinica, tecnologica e strutturale nell'assistenza del paziente complesso. Data consultazione online 16/01/2020

15. Ministero della Salute http://www.archeo.salute.gov.it/ data consultazione on line 15/01/2020.

16. https://www.quotidianosanita.it/studi-e-analisi/articolo. php?articolo_id $=17658 /$ data consultazione on line $18 / 01 / 20020$

17. Freddi G, Romàn-Pumar JL. Evidence-based medicine:what it can and cannot do. Ann Ist Super Sanità 2011; Vol. 47, No. 1: 22-25.

18 Wieringa S, Engebretsen E, Heggen K, Greenhalgh T. Has evidence-based medicine ever been modern? A Latour-inspired understanding of a changing EBM. J Eval Clin Pract. 2017 Oct;23(5):964-970.

19 Edwards A, Elwyn G. Shared decision-making in health care: achieving evidence-based patient choice. 2009:310. eBook. Oxford University Press.

20. Ekman I, Swedberg K, Taft C, et al. Person-centered care-ready for prime time. Eur J Cardiovasc Nurs. 2011;10(4):248-5.

21. Vandvik PO, Otto CM, Siemieniuk RA, et al. Transcatheter or surgical aortic valve replacement for patients with severe, symptomatic, aortic stenosis at low to intermediate surgical risk: a clinical practice guideline. BMJ. 2016; 354

22. Elwyn G, Quinlan C, Mulley A, et al. Trustworthy guidelines - excellent; customized care tools- even better. BMC Med. 2015;13:199.

23. Elwyn G, Lloyd A, May C et al. Collaborative deliberation: a model for patient care. Patient Educ Couns. 2014 Nov;97(2):158-64.

24. Gilioli A. Ridateci i medici che sanno fare diagnosi: oggi per il paziente è via crucis tra gli specialisti. Da L'Espresso, 7 dicembre 2017. Data consultazione online 18/01/2020.

25. Fontanella A. Noi, medici internisti, siamo i veri dottor House della medicina italiana. Da L'espresso, 14 dicembre 2017. Data consultazione on line 18/01/2020. 


\title{
Metodologia clinica e EBM: vantaggi e limiti nel mondo reale dei pazienti complessi - Trappole e possibili bias
}

\author{
Maurizio Cavalleri \\ Medicina Interna, Ospedale Sestri Levante (GE), Italia
}

\section{Introduzione}

La valutazione critica della letteratura scientifica (critical appraisal) rappresenta il cardine attorno cui ruota tutto il ragionamento della medicina basata sulle evidenze.

Il critical appraisal è infatti lo strumento che ci permette di di identificare e selezionale le migliori evidenze nel mare magnum della letteratura scientifica in modo da poterle poi trasferire nella pratica clinica secondo il principio dell'evidence-based-practice.

Nel gennaio 2014 The Lancet ha pubblicato la serie Research: Increasing Value, Reducing Waste documentando che per aumentare il ritorno degli investimenti della ricerca (value) è necessario stabilire priorità più rilevanti, migliorare disegno, conduzione e analisi, ottimizzare le procedure di gestione e regolamentazione, garantire un adeguato reporting e una migliore usabilità della ricerca. È stata quindi costituita la REWARD (REduce research Waste And Reward Diligence) Alliance e lanciata la campagna Lancet-REWARD che ha pubblicato il REWARD Statement e le raccomandazioni con relativi indicatori di monitoraggio su cinque aree di potenziali sprechi della ricerca biomedica: rilevanza della ricerca, adeguatezza del disegno dello studio, dei metodi e delle analisi statistiche, efficienza dei processi di regolamentazione e gestione, completa accessibilità ai dati, usabilità dei report.

Nello specifico in questa sede ci occupiamo di uno di questi 5 elementi chiave delle letteratura scientifica: il rigore nella conduzione metodologica.

Corrispondente: Maurizio Cavalleri, Medicina Interna, Ospedale Sestri Levante (GE), Italia.

E-mail: mcavalleri.doc@hotmail.it

Articolo pubblicato secondo la Creative Commons Attribution NonCommercial 4.0 License (CC BY-NC 4.0).

${ }^{\circ}$ Copyright: the Author(s), 2020

Licensee PAGEPress, Italy

QUADERNI - Italian Journal of Medicine 2020; 8(1):46-48
Le dimensioni che condizionano la valutazione critica della letteratura scientifica primaria (ovvero studio osservazionali e studi sperimentali) sono tre: la validità interna, la rilevanza clinica e l'applicabilità.

Queste dimensioni di fatto si applicano anche alla ricerca secondaria, in particolare alle revisioni sistematiche e meta-analisi, per le quali però va considerata una quarta dimensione: la consistenza.

In questo capitolo della monografia tratteremo specificamente il tema della validità interna degli studi primari.

Sinonimo di validità interna è validità metodologica il cui scopo è quello di ridurre al minimo gli errori sistematici (BIAS) che possono inficiare i risultati dello studio.

La validità interna è direttamente proporzionale al rigore metodologico seguito dai ricercatori nella pianificazione, conduzione dello studio e analisi dei dati.

Definiamo innanzitutto cosa intendiamo per BIAS. Un errore casuale è un errore dovuto al caso, che può verificarsi o no in modo casuale, quindi imprevedibile. Replicazioni multiple della stessa misurazione producono differenti risultati in tutte le direzioni per variazioni casuali ma la media da il risultato corretto.

$\mathrm{Al}$ contrario il BIAS è un errore sistematico dovuto ad un difetto metodologico intrinseco allo studio e quindi riproducibile e prevedibile, appunto in modo sistematico. Il bias di conseguenza comporta una distorsione sistematica delle rilevazioni in modo sbilanciato a favore del braccio sperimentale o di quello di controllo, rendendo quindi meno attendibile e significativo il risultato dello studio. In questo caso replicazioni multiple della stessa misurazione producono risultati sempre nella stessa direzione.

Lo strumento cardine per la valutazione del rischio di BIAS nei trial clinici randomizzati controllati è il Cochrane collaboration tool del 2011.

Questo strumento sostanzialmente si articola in 6 dimensioni cioè nella valutazione di 6 tipi di bias che possono presentarsi nei vari step della conduzione di un trial.

La prima dimensione descrive il cosiddetto selection Bias cioè il bias che si può verificare nella 
selezione dei pazienti: questo bias descrive differenze sistematiche nelle caratteristiche basali dei pazienti arruolati nei gruppi di studio. Tale dimensione si ripartisce ulteriormente in due items definiti Random sequence generation e allocation concealment.

\section{Random sequence generation}

L'investigatore dovrebbe innanzitutto descrivere accuratamente la modalità con cui viene generata la sequenza di randomizzazione. Ad esempio se viene dichiarato che è stato usato un software informatico, lancio dei dadi etc.. questo è una garanzia che effettivamente la sequenza è random. Viceversa se ad esempio viene usato il numero sequenziale di cartella clinica, giorni di ricovero a altre modalità analoghe, questo può essere oggetto di distorsioni e non rappresenta una reale randomizzazione

\section{Allocation concealment}

Nei trial controllati e randomizzati (RCTs) il concetto di mascheramento/occultamento/cecità entra in gioco in due diversi momenti. Prima della randomizzazione l'occultamento della lista (allocation concealment) ha l'obiettivo di prevenirne il sovvertimento e il conseguente bias di selezione. Dopo la randomizzazione, la cecità (blinding) impedisce a una o più categorie di soggetti coinvolti nel trial di conoscere il trattamento assegnato.

In tutti i trial l'occultamento della lista di randomizzazione è sempre possibile ed efficace nel prevenire il bias di selezione. Viceversa, il blinding non è sempre attuabile per motivazione etiche e/o pratiche (open trial), e la sua efficacia nel prevenire i bias dipende da numerose variabili.

Il CONSORT statement sottolinea la necessità di riportare sia il processo di allocation concealment sia quali soggetti sono blinded, nonché le strategie utilizzate per attuare il blinding.

Sul concetto di singolo, doppio o triplo cieco le definizioni in letteratura non sono univoche e questo crea spesso poca chiarezza per cui il CONSORT statement sottolinea la necessità di identificare quali soggetti sono blinded, abbandonando la classica terminologia (singolo, doppio, triplo cieco) poco riproducibile e di conseguenza ambigua.

Essere a conoscenza del trattamento assegnato può influenzare, consciamente o senza volerlo, il giudizio di varie categorie di soggetti coinvolti nel trial.

Participants: soggetti randomizzati.

Healthcare providers: medici, infermieri, fisioterapisti e altri professionisti che assistono i partecipanti e/o somministrano l'intervento.

Data collectors: professionisti che raccolgono i dati (segni, sintomi, questionari, etc.); possono identificarsi con gli healthcare providers e/o con gli outcomes assessors.

Outcomes assessors: professionisti che hanno il compito di definire se il partecipante ha raggiunto, $o$ meno, l'outcome di interesse.

Data analysts: statistici che analizzano i dati.

Data safety and monitoring committee: comitato che rivede i dati sulla sicurezza-efficacia dei trattamenti.

Writers: autori del manoscritto.

Il CONSORT Statement raccomanda (item 11) che gli Autori dovrebbero: i) riportare quali soggetti sono blinded: partecipanti, professionisti che erogano l'assistenza, valutatori degli esiti, statistici che analizzano i dati; ii) riportare quali soggetti non sono blinded, specificando le motivazioni; iii) descrivere le tecniche di blinding incluse le variabili (odore, colore, sapore, consistenza, etc.) che rendono apparentemente identici i trattamenti; iv) documentare le modalità per verificare il successo del blinding.

L'impossibilità di attuare il blinding - o il suo insuccesso - introducono due principali bias che possono sovrastimare in maniera variabile l'efficacia dei trattamenti.

\section{Performance bias}

Consiste nella differenza sistematica dell'assistenza erogata ai due gruppi di pazienti, conoscendo quale trattamento è stato assegnato. Anche se la standardizzazione del protocollo non dovrebbe permetterlo è frequente, ad esempio, il concomitant treatment bias, somministrazione di altre terapie che riducono la probabilità dell'esito nel gruppo dei trattati e/o la aumentano in quello dei controlli. Il performance bias si previene mantenendo blinded partecipanti e healthcare providers.

\section{Detection bias (ascertainment bias)}

Il bias di accertamento dell'esito consegue alla possibilità che l'outcomes assessor sia condizionato dal conoscere quale trattamento riceve il paziente. Negli studi che misurano outcome soggettivi riportati dal paziente - è anche definito reporting bias. Viene prevenuto mantenendo blinded partecipanti, outcomes assessors e possibilmente anche healthcare providers. All'aumentare dell'oggettività dell'outcome, diminuisce la probabilità del detection bias, che di fatto scompare se l'outcome è... la morte del paziente.

Idealmente un trial dovrebbe mantenere blinded tutte le categorie di soggetti coinvolti; se questo obiettivo è facilmente raggiungibile negli studi che confrontano una terapia farmacologica con il placebo, 
alcuni trial che prevedono un controllo attivo richiedono specifiche strategie.

Se lo studio confronta due terapie farmacologiche con differente modalità/via di somministrazione è necessario prevedere il doppio placebo (double dummy): ad esempio, al gruppo dei trattati viene somministrato il farmaco orale e il placebo per via parenterale, mentre al gruppo di controllo placebo per os e farmaco attivo per via parenterale.

Quando uno o entrambi i trattamenti non sono mascherabili (chirurgia, terapie interventistiche, terapie manuali, etc.), partecipanti e healthcare providers sono inevitabilmente a conoscenza del trattamento. In questi studi è possibile effettuare una valutazione in cieco degli outcome, attraverso professionisti che non conoscono il trattamento ricevuto dal paziente (outcomes assessment committee). Questa modalità può essere utilizzata solo se l'esito è valutato sui risultati di indagini strumentali e non direttamente sul paziente, la cui presenza potrebbe contaminare il giudizio degli outcomes assessors.

Infine, per gli outcome di laboratorio che non presentano margini di rilevazione soggettiva, l'utilizzo di strumenti accurati e ben calibrati previene il bias di accertamento dell'esito.

\section{Attrition bias/incomplete outcome data}

Seguendo idealmente la sequenza di sviluppo di un trial dopo la randomizzazione e l'esposizione ai trattamenti segue il periodo di follow-up nel quale alcuni Pazienti possono uscire dallo studio perché deceduti, per progressione o altre cause specifiche.

Altri Pazienti invece possono essere semplicemente persi al follow -up cioè potremmo non avere più informazioni su di loro (persi al follow-up). Non sapremmo quindi se sono usciti dallo studio e perché, se per cause imputabili al trattamento o no.

In generale non ci sono cut-uff standard numerici di percentuali di persi al follow-up per definire se il rischio di bias per questa dimensione è alto o basso (in generale comunque se i follow-up sono più del $20 \%$ il rischio è alto).
I criteri da valutare per definire se alto o basso rischio sono se i persi al follow-up sono bilanciati o no tra i 2 bracci di trattamento, se sono riportate le cause della perdita (non differenti nei bracci e non imputabili al trattamento) e se viene effettuata l'analisi statistica per Intention to treat e non solo per protocol.

\section{Selective reporting bias}

Quest'ultima dimensione valuta l'effettiva trasparenza nella pubblicazione di tutti i risultati, sia quelli positivi che negativi.

Innanzitutto dovrebbe essere disponibile il protocollo dello studio, pubblicato antecedentemente allo studio sui registri appositi come clinicaltrial.gov.

Secondariamente il revisore dovrebbe verificare che i risultati dello studio riportino in modo completo i risultati di tutti gli outcome predefiniti nella sezione metodi del protocollo dello studio

I risultati non significativi potrebbero essere riportati parzialmente (dati solo su alcuni sottogruppi, solo alcuni follow up, solo alcune scale, solo alcune parti di scale, reporting incompleto, etc.)

In tutti questi casi si parla di un alto rischio di selective reporting bias.

Quelle descritte sono le 6 dimensioni che definiscono la validità interna /metodologica nella conduzione di uno studio.

La valutazione del rischio di bias è uno strumento che può essere utilizzato per la valutazione del singolo studio o, più spesso, nell'ambito di una revisione sistematica in cui il risk of bias rappresenta il primo dominio di una più completa e complessa valutazione globale della qualità dell'evidenza su uno specifico quesito. In questo caso la valutazione è outcomecentrica cioè per ogni outcome considerato nel quesito della revisione si fa una valutazione del rischio di bias dei singoli studi (giudizio che può variare da outcome a outcome, come nel caso del giudizio sul performance bias per un outcome soggettivo rispetto ad un outcome oggettivo). 


\title{
Significatività statistica e rilevanza clinica
}

\author{
Daniela Tirotta \\ UO Medicina Interna, Ospedale Cervesi di Cattolica, AUSL Romagna, Italia
}

\section{Premessa}

L'incertezza è un problema costante in medicina, anche se la sua importanza è spesso sottovalutata e le sue conseguenze trascurate: ${ }^{1}$ alberga nella diagnosi differenziale di una patologia complessa, così come nella creazione di nuove linee guida da parte di una società scientifica (esempio ne è la ricerca continua di nuovi target pressori, così come l'approccio diagnostico alla neoplasia mammaria, che oscilla costantemente tra l'over e l'under diagnosis).

L'attuale rigore metodologico della sperimentazione clinica ha prodotto dati sicuramente più affidabili, ma il concetto della significatività statistica può creare interpretazioni errate.

\section{Esempio}

In un recente trial sul trattamento adiuvante del cancro al seno. ${ }^{2}$ Gli Autori concludono che gli inibitori dell'aromatasi hanno portato a tassi significativamente più alti di sopravvivenza libera da malattia, sebbene il tasso di sopravvivenza globale non sia più alto.

Il significativamente più alto corrisponde al $4 \%$. Siamo davvero sicuri che questa percentuale corrisponda ad un beneficio tangibile per il paziente?

\section{Non è sbagliato il disegno dello studio, ma il linguaggio utilizzato per riportare i risultati}

La questione non è trascurabile, poiché molte pratiche mediche sono state accettate più per la difesa di figure di spicco, che per un attento studio. In un

Corrispondente: Daniela Tirotta, UO Medicina Interna, Ospedale Cervesi di Cattolica, AUSL Romagna, Italia. E-mail: danitirotta@libero.it

Articolo pubblicato secondo la Creative Commons Attribution NonCommercial 4.0 License (CC BY-NC 4.0).

${ }^{\circ}$ Copyright: the Author(s), 2020

Licensee PAGEPress, Italy

QUADERNI - Italian Journal of Medicine 2020; 8(1):49-57 articolo $^{3}$ Prasad e Colleghi hanno rivisto tutti gli articoli originali pubblicati in una rivista ad alto impatto tra il 2000 e il 2010 ed hanno trovato che circa il $40 \%$ dei 363 articoli che testano lo standard di assistenza ha comportato un'inversione delle tendenze della pratica medica.

Probabilmente la maggior parte di queste inversioni avrebbe potuto essere evitata, se l'incertezza inerente la pratica consolidata fosse stata riconosciuta e contestualizzata in base alla forza delle prove.

Come possiamo essere più schietti sull'incertezza ed evitare l'overconfidence? Interpretando gli studi, tenendo conto della qualità delle evidenze e della loro dimensione, come succede con il metodo Grade nella stesura delle linee guida.

\section{Cosa si intende per significatività statistica}

Negli studi clinici leggiamo spesso che la soglia della significatività statistica ha un valore di 0,05 . Ma cosa significa e perché questa soglia convenzionale?

Immaginiamo di formulare un'ipotesi scientifica che asserisce che il valore di colesterolemia è più basso nei soggetti trattati con un nutraceutico che nei soggetti trattati con placebo.

La corrispondente ipotesi nulla è che il valore di colesterolemia sia uguale nei trattati con nutraceutico e nei trattati con placebo (cioè la differenza trai 2 bracci sia nulla).

Supponiamo che i due gruppi siano di 40 pazienti ciascuno, randomizzati per nutraceutico o placebo; alla fine di un anno di trattamento, la colesterolemia media del primo gruppo è 194,4 (DS 15,6) mg/dL, mentre quella del gruppo di controllo è 202,1 (DS $14,8) \mathrm{mg} / \mathrm{dL}$. Per confrontare due medie, calcolate su due campioni indipendenti, il test statistico appropriato è la $t$ di student.

Utilizzando il valore con un foglio di calcolo per la $\mathrm{t}$ di student, il valore di $\mathrm{P}$ che risulta è $0,026(<0,05)$, ovvero il risultato è statisticamente significativo.

Ma cosa significa? Significa che sotto l'ipotesi nulla, cioè se non esistono differenze tra soggetti trattati e non trattati, la probabilità di un risultato come quello ottenuto o più estremo è pari a 26 su 1000 , cioè piuttosto improbabile. 
Concludiamo, dunque, che è poco probabile che la differenza riscontrata tra i due gruppi sia dovuta al caso.

In sintesi, più piccolo è il valore di $P$, più grande è l'evidenza contro l'ipotesi nulla, dichiarata non plausibile; è improbabile, cioè, che si sia realizzata per effetto del caso, e quindi rifiutata.

In caso, invece, di un P elevata $(>0,05)$, l'ipotesi nulla non viene rifiutata, ma l'informazione che si ricava è che non vi sono dati sufficienti per una conclusione.

Fu Fisher a proporre la p come un indicatore della discrepanza tra i dati e l'ipotesi nulla negli anni ' $20 .{ }^{4}$ Un decennio più tardi, Neyman e Pearson hanno sviluppato il metodo che da loro prende il nome, con lo scopo di sostituire l'interpretazione soggettiva della significatività statistica con un processo decisionale. ${ }^{4}$

\section{Esempio}

Nel nostro esempio relativamente al confronto tra l'ipotesi nulla e l'ipotesi alternativa, in merito alla colesterolemia, il risultato del test d'ipotesi (secondo Neyman e Pearson) è una decisione, non un'inferenza. La decisione che non si può asserire che esista differenza tra la colesterolemia del gruppo dei trattati con nutraceutici e quella dei trattati con placebo (mancato rifiuto dell'ipotesi nulla) o asserire che la differenza esiste (rifiuto dell'ipotesi nulla) espone il ricercatore al rischio di due tipi di errore: i) un risultato falsamente positivo, o errore di tipo I: comportarsi come se vi fosse una differenza tra le due terapie, quando di fatto non vi è (rifiutare l'ipotesi nulla quando essa è vera); ii) un risultato falsamente negativo, o errore di tipo II: concludere che le due terapie non differiscono, quando di fatto sono diverse (ovvero non rifiutare l'ipotesi nulla quando l'ipotesi alternativa è vera). Più usato è il complemento a 1 dell'errore di tipo II (1-beta), definito potenza.

Neyman e Pearson dichiarano che non si è in grado di valutare lo stato di realtà basandosi su un singolo esperimento, con una $P$, mentre Goodman, ${ }^{2}$ ha paragonato questo processo a quello in un sistema giudiziario che non focalizza la sua attenzione su quale accusato sia colpevole o innocente, ma cerca di controllare il numero di sentenze erronee (nel lungo termine, non sbaglieremo molto spesso).

Nella pratica statistica si è quindi generato una sorta di metodo ibrido in cui: i) prima dell'esperimento, si fissa il livello di errore di tipo I (praticamente sempre il 5\%); ii) sempre prima dell'esperimento, si fissa la potenza (che è il complemento a 1 dell'errore di tipo II), praticamente sempre fissata all' $80 \%$; iii) si calcola quindi il valore $P$ e si rifiuta l'ipotesi nulla se tale valore è inferiore al valore prefissato di errore di tipo I .

Importante sottolineare che il test di significatività può portare a rifiutare l'ipotesi nulla, ma non può mai dimostrarla o confermarla. Ciò implica la scorrettezza metodologica degli studi che, basandosi sull'assenza di significatività statistica, concludono per l'equivalenza tra due gruppi. Un esempio è rappresentato da uno studio recente che confronta 3 tecniche per l'elettroforesi delle proteine: ${ }^{5,6}$ gli autori non ottengono la significatività statistica $(\mathrm{P}>0,05)$ e concludono che le caratteristiche analitiche sono equivalenti. Il mancato rifiuto dell'ipotesi nulla, tuttavia, permette di affermare che i dati non consentono di rifiutare l'ipotesi nulla (di equivalenza trai metodi), e non di concludere che l'ipotesi nulla è vera e quindi che i 3 metodi possono essere considerati equivalenti.

\section{L'intervallo di confidenza}

L'enfasi sulla significatività statistica può portare, se disattenti, a leggere in maniera distorta gli studi. Per risolvere questa problematica, è stato introdotto l'intervallo di confidenza; i) se l'IC include il non effetto (pari a 0 per una differenza o 1 per un rapporto), ciò implica l'assenza di una significatività statistica; ii) se, invece, per esempio l'intervallo di confidenza ha come limite inferiore 0,024 , per cui lo zero (valore di non effetto) non è incluso nell'intervallo dei valori possibili, si raggiunge la significatività statistica; iii) nel caso, invece, il limite inferiore dell'intervallo di confidenza sia pari a $-0,03$, la scarsa distanza del limite stesso dallo zero sta a suggerire che non ci si trova molto distante dal raggiungere la significatività statistica. In queste situazioni occorre tenere sempre presente il ruolo della potenza statistica (quindi della dimensione campionaria). Aumentando, infatti, il campione, non solo si ridurrà la $\mathrm{P}$, ma si ridurrà anche l'IC, distribuendosi sempre in modo simmetrico attorno alla media.

La $\mathrm{P}$ non va mai valutata a prescindere dal contesto:

- Valori simili di P devono portare a conclusioni simili, non diametralmente opposte.

Nel confronto tra due gruppi di 100 pazienti randomizzati a trattamento con nutraceutico o placebo, il colesterolo plasmatico dopo un anno di trattamento è 197,07 (DS 14,7) $\mathrm{mg} / \mathrm{dL}$ nel primo gruppo e 201,14 (DS 14,7) nel gruppo di controllo; la t è pari a 1,963 , che corrisponde a un valore di $\mathrm{P}=0,051$. Il valore è più alto della soglia di 0,05 , per cui non possiamo rifiutare l'ipotesi nulla e non possiamo asserire che vi sia differenza tra i due gruppi. La dimensione dell'effetto, cioè la differenza nella colesterolemia, è $4,07 \mathrm{mg} / \mathrm{dL}$.

Immaginiamo di ripetere l'esperimento in condizioni analoghe e di ottenere una P di 0,049, che consente di rifiutare l'ipotesi nulla. Siamo sicuri che i due studi portino a conclusioni opposte? 
- Il valore di P non dovrebbe mai essere riportato senza inserire anche la dimensione dell'effetto osservato: un effetto di piccola dimensione in uno studio con un'ampia dimensione campionaria può risultare nel medesimo valore di P di un effetto importante in uno studio di piccole dimensioni.

Consideriamo due studi che confrontano pazienti trattati con nutraceutico con un gruppo di controllo trattato con placebo; entrambi gli studi riportano risultati statisticamente significativi e per entrambi la P è pari a 0,0009: i) nel primo studio, i soggetti trattati con nutraceutico hanno una colesterolemia di 197,14 (DS 14,65), mentre il gruppo in placebo ha 201,14 (DS 14,60) mg/dL; la t risulta 3,349 . Reclutati 600 soggetti; ii) nel secondo studio, il gruppo in nutraceutico ha colesterolemia 196,81 (DS 13,28), mentre quello in placebo 205,38 (DS 11,62) mg/dL; la t risulta 3,434. Reclutati 100 soggetti. Se andiamo, tuttavia, a esaminare la dimensione dell'effetto, cioè la differenza nei livelli di colesterolo, questa è 4,0 $\mathrm{mg} / \mathrm{dL}$ nel primo studio (di alta numerosità), mentre è $8,57 \mathrm{mg} / \mathrm{dL}$, quindi più che doppia, nel secondo studio (di minor numerosità).

\section{Cosa si intende per significatività clinica}

Abbiamo parlato fino ad ora di significatività statistica, ma implica per forza significatività clinica?

Esempio 1: Per esemplificare il concetto, immaginiamo unTRC ben condotto in cui si conclude che, indossando un casco, si ridurrebbe dell' $80 \%$ la probabilità di essere colpiti da un fulmine a ciel sereno. Indossereste il casco?

Nell'interpretazione del quesito clinico è essenziale considerare il contesto in cui è posto.

Esempio 2: Nello studio di confronto tra nutraceutico e placebo immaginiamo che la dimensione dell'effetto, che rappresenta l'informazione rilevante per il clinico, sia una differenza di $7,7 \mathrm{mg} / \mathrm{dL}$, con un valore di P molto piccolo (es. 0,0001), ciò significa che la differenza fra i valori di colesterolemia è più significativa?

Occorre, in realtà fissare l'attenzione sulla differenza tra le due medie: una differenza di 7,7 $\mathrm{mg} / \mathrm{dL}$ di colesterolemia è clinicamente rilevante? Non è la dimensione di $\mathrm{P}$ che risponde a questa domanda. La risposta va chiesta all'esperto dello specifico settore clinico, non alla statistica. Molti di noi riterrebbero che una differenza di $7,7 \mathrm{mg} / \mathrm{dL}$ di colesterolemia non sia clinicamente rilevante.

Nell'interpretazione di un articolo, dunque, dopo aver parlato di affidabilità dei risultati, il successivo quesito è: l'effetto ottenuto con l'intervento è grande o piccolo?

Infatti la significatività statistica non coincide necessariamente con la rilevanza clinica, perché non coincide con la rilevanza clinica/organizzativa dell'intervento stesso.

Le dimensioni dell'effetto considerato attengono, infatti, una serie di variabili, tra cui: i) gli eventi avversi clinici, economici ed organizzativi che l'intervento può generare; ii) le preferenze del paziente e degli operatori; iii) il fastidio o le resistenze che l'intervento potrebbe provocare; iv) il contesto in cui l'intervento si applica.

\section{Gli 11 step della check-list della significatività}

La premessa è che il raggiungimento di un outcome primario positivo non basta ad accontentarsi per far adottare tout court un nuovo farmaco e modificare la pratica clinica. Un dato studio verrà passato al vaglio da schiere di esperti clinici, redattori di linee guida, autorità regolatorie, medici, membri di società scientifiche e associazioni di pazienti prima di decidere se un nuovo trattamento verrà approvato 0 meno. Ma intanto, per capire se i risultati di un certo studio sono così interessanti da preludere a probabili ricadute sulla pratica clinica, è bene andare a leggere in maniera più approfondita i risultati, ponendosi queste 11 domande tecniche, 11 step, come pubblicato su un recente lavoro del New England. ${ }^{5}$

\section{Step 1: Il primo step è correlato al concetto già espresso di significatività statistica: un valore della $\mathbf{P}<0,05$ è abbastanza buono?}

Una $\mathrm{P}<0,05$ significa che c'è una probabilità del $5 \%$ di un risultato falso positivo (ovvero di una non differenza di risultati trai due bracci di trattamento). Dunque, se si vuole avere un risultato al di là di ogni ragionevole dubbio, è bene puntare ad una $\mathrm{P}$ molto inferiore (ad esempio $<0,001){ }^{5}$

\section{Esempio}

In linea con questo criterio i risultati dello studio PARADIGM-HF, ${ }^{7}$ nel quale l'associazione sacubitrilvalsartan confrontata con l'enalapril ha raggiunto l'endpoint primario (end-point composito di mortalità cardiovascolare e ricoveri per scompenso cardiaco) con una $\mathrm{P}<0,00001$ e questo ha portato all'approvazione del sacubritril-valsartan da parte delle autorità regolatorie.

Al contrario, nello studio SAINT I5 di NXY-059, ${ }^{8}$ che confrontava un agente anti radicali liberi, rispetto al placebo per il trattamento dell'ictus ischemico acuto, il valore $\mathrm{P}$ per l'esito primario della disabilità a 90 giorni era 0,038 , pertanto non una forte evidenza di efficacia. è stato necessario un secondo studio più ampio (SAINT II6), che non ha rivelato alcun effetto significativo $(\mathrm{P}=0,33)$, ed ha spinto gli autori a concludere che NXY-059 è inefficace per il trattamento dell'ictus ischemico acuto. 
Step 2: Dal secondo step in poi si analizza, invece, la significatività clinica

Qual è l'ordine di grandezza del beneficio derivante da un determinato trattamento? $\mathrm{Al}$ di là della significatività statistica, un'altra fondamentale questione da porsi è la rilevanza clinica dei risultati dello studio; per questo è necessario calcolare gli effetti del trattamento in scala relativa (relative risk o hazard ratio) e assoluta (calcolando ad esempio le differenze nel tasso di eventi che si verificano nel follow-up e il number needed to treat).

\section{Esempio 1}

Nello studio IMPROVE-IT, ${ }^{9}$ ezetimibe è stato confrontato con placebo in pazienti con sindromi coronariche acute che erano in trattamento con simvastatina; l'hazard ratio per l'outcome primario composito di morte cardiovascolare, infarto del miocardio, angina instabile, rivascolarizzazione, ictus era 0,94 (intervallo di confidenza al $95 \%$ [IC], da 0,89 a 0,$98 ; \mathrm{P}=0,016$ ). I tassi di eventi primari a 7 anni erano del $32,7 \%$ con ezetimibe rispetto al $34,7 \%$ con placebo, una differenza di 2 punti percentuali, con un IC $95 \%$, che variava da quasi 0 a 4 punti percentuali.

Cosa ci dicono i risultati dello studio quindi?

Sebbene i risultati di questo studio siano stati descritti come positivi, ci si potrebbe chiedere se il beneficio di ezetimibe sia abbastanza grande da giustificarne il costo e le potenziali complicazioni. Un comitato consultivo della Food and Drug Administration (FDA) ha raccomandato di non espandere l'indicazione di ezetimibe alla riduzione degli eventi cardiovascolari.

È opportuno ribadire che non vi è correlazione tra grandezza della $\mathrm{P}$ e grandezza dell'effetto associato all'evento. Un'importante significatività statistica, cioè, non implica, a prescindere, una maggiore significatività clinica.

\section{Esempio 2}

Immaginiamo 2 trial che confrontino l'effetto di un farmaco A e di un farmaco B con il placebo nella riduzione della pressione arteriosa in presenza di cardiopatia ipertensiva. Il farmaco A riduce la pressione rispetto al placebo con una $\mathrm{P}=0.0001$, anche il farmaco $\mathrm{B}$, ma con una $\mathrm{P}=0.05$. Il lettore può essere più sicuro che la differenza ottenuta con il farmaco $\mathrm{A}$ non sia dovuta al caso, ma in nessun modo deve interpretare i dati come una dimostrazione di maggiore potenza o efficacia del farmaco A rispetto a B.

Step 3: L'outcome primario è rilevante dal punto di vista clinico (ed internamente consistente)?

Sebbene gli studi di fase 3 abbiano in genere una potenza sufficiente a raggiungere dei risultati di rilevanza clinica, per alcune patologie ci si accontenta dei cosiddetti outcome surrogati (es. riduzione dell'emoglobina glicata come misura surrogata dell'effetto anti-glicemico nei soggetti con diabete). Ma i risultati di alcuni grandi trial hanno messo in seria discussione l'affidabilità di questi endpoint surrogati.

\section{Esempio}

Esempi di outcome surrogati: i) riduzione della massa di un tumore invece della mortalità; ii) riduzione della pressione arteriosa invece della mortalità totale o cardiovascolare o dei casi di infarto; iii) riduzione di un marcatore tumorale (es. PSA) invece della mortalità; iv) riduzione della glicemia nei diabetici invece della riduzione della mortalità o di complicanze gravi.

Esempi applicati a studi clinici:

Nello studio ACCORD, ${ }^{10}$ il braccio trattato in maniera più intensiva, rispetto a quello della terapia standard, otteneva una maggiore riduzione dell'emoglobina glicata. Peccato però che era anche gravato da una maggior mortalità.

Studio CAST, ${ }^{11}$ nel quale sono stati usati 3 antiaritmici (flecainide, moricizina ed encainide) in pazienti con contrazioni ventricolari premature (CVP), a loro volta predittive di morte cardiaca improvvisa.

I 3 farmaci hanno ridotto le CVP, dimostrandosi quindi efficaci. La sperimentazione è però stata bloccata perché a 10 mesi morivano di più i pazienti in terapia antiaritmica. Questo perché i farmaci agivano sull'esito surrogato, ma non su quello rilevante, cioè la mortalità: riducevano i casi di CVP, ma aumentavano il rischio di altre aritmie letali.

\section{Outcome multipli o compositi}

Di fronte, invece, ad un outcome composito, gli Autori ${ }^{5}$ raccomandano una lettura separata delle sue diverse componenti, per capire qual è quella che ha maggiormente generato il risultato.

Gli outcome multipli o compositi comprendono più esiti, che vengono valutati in maniera cumulativa. Per esempio, si valuta se un dato farmaco riduce il colesterolo, riduce i casi di ospedalizzazione da cardiopatia ischemica e la mortalità cardiovascolare. Così facendo è più facile dimostrare l'efficacia di un trattamento, è quindi sempre bene diffidare di un esito composito, a meno che i diversi esiti compresi non siano tutti rilevanti per il malato.

Supponiamo che gli autori pubblichino un lavoro, in cui valutano un esito, che però non era stato definito a priori come esito primario dello studio, per esempio, accorgendosi al termine della ricerca che il farmaco riduce la pressione, ma non la mortalità, indicano 
come esito dello studio la riduzione dei valori pressori, mentre lo studio era stato concepito per valutare la mortalità. Come ci si può accorgere di ciò? Da qualche anno è stato imposto l'obbligo di pubblicare i disegni degli studi nelle banche dati internazionali prima di iniziare la sperimentazione.

\section{Esempio}

Nel trial RITA- $3,{ }^{12}$ che ha valutato gli effetti del trattamento interventista rispetto ad uno conservativo in una popolazione di pazienti con sindrome coronarica acuta, un numero inferiore di pazienti del gruppo con trattamento interventista ha presentato l'endpoint primario, un composito di mortalità, infarto del miocardio, angina refrattaria a 4 mesi. Un risultato tuttavia trascinato dal dimezzamento dei casi di angina refrattaria, mentre nessuna differenza era stata rilevata nei tassi di infarto o di mortalità. Sulla base di questo studio non era dunque possibile concludere che la strategia interventista fosse un intervento salvavita. Ma in seguito uno studio con un follow-up di 5 anni ha evidenziato una riduzione del $22 \%$ nel tasso di infarti e mortalità tra i pazienti del gruppo di intervento, dato confermato anche da successive metanalisi. Tutto ciò ha dunque portato ad adottare la rivascolarizzazione coronarica precoce nei soggetti con sindromi coronarie acute per migliorarne la prognosi. Analogo il risultato di alcune metanalisi. ${ }^{13,14}$

\section{Step 4: Gli outcome secondari sono d'aiuto?}

L'impressione che i risultati di uno studio siano positivi viene rinforzata dalla presenza di outcome secondari prespecificati che mostrino anch'essi un beneficio del trattamento in esame. Al contrario se gli outcome secondari risultano negativi, si insinua il dubbio della validità dell'intero studio.

\section{Esempio}

Nello studio SAINT I5 ${ }^{15}$ sull'NXY-059 nell'ictus ischemico acuto, non esistevano prove di beneficio per due outcome secondari chiave: i punteggi sulla National Institutes of Health Stroke Scale e sull'indice di Barthel. Questa assenza di prove ha aumentato il sospetto riguardo all'outcome primario positivo, un sospetto che è stato rafforzato dal risultato negativo nello studio sequel, SAINT II. ${ }^{16}$

Al contrario, nello studio EMPA-REG OUTCOME ${ }^{17}$ di empagliflozin rispetto al placebo nel diabete di tipo 2, il beneficio di empagliflozin rispetto all'outcome primario composito (morte cardiovascolare, infarto del miocardio o ictus) era di significato borderline, con un rapporto di rischio di 0,86 (IC al 95\%, da 0,74 a 0,99; $\mathrm{P}=0,04$ ). Tuttavia, questo risultato è stato guidato da un tasso decisamente più basso di morte cardiovascolare (hazard ratio, 0,62; IC al 95\%, da 0,49 a 0,77; $\mathrm{P}<0,001)$ ed è stato rafforzato da risultati simili per quanto riguarda la morte per tutte le cause $(\mathrm{P}<0,001)$ e i ricoveri per insufficienza cardiaca $(\mathrm{P}=0,002)$. Pertanto, l'effetto di empagliflozin è stato osservato principalmente nei secondari, sebbene l'esito positivo per il risultato primario abbia conferito credibilità iniziale.

\section{Step 5: I risultati dello studio sono confermati per tutti i sottogruppi importanti?}

Gli effetti di un farmaco: i) possono essere diversi a seconda delle caratteristiche dei pazienti; ii) oppure essere più evidenti in alcuni sottogruppi ad alto rischio, come nel caso delle statine nei soggetti con diversi fattori di rischio cardiovascolare (è questo il motivo per cui le statine in prevenzione primaria vengono riservate ai pazienti ad alto rischio); iii) più complesso è il caso di un trial positivo a favore di un farmaco, nell'ambito del quale però l'analisi per sottogruppi non evidenzia un beneficio dall'assunzione di questa terapia. La prudenza è d'obbligo nell'interpretare i risultati delle analisi per sottogruppi. Ė anche ragionevole però risparmiare ad alcuni pazienti un trattamento probabilmente per loro inefficace o potenzialmente dannoso.

\section{Esempio}

Nello studio PLATO, ${ }^{18,19}$ che ha coinvolto pazienti con una sindrome coronarica acuta, il rischio di outcome primario composito di morte cardiovascolare, infarto del miocardio o ictus era inferiore del $16 \%$ con ticagrelor rispetto a clopidogrel nella popolazione complessiva dello studio $(\mathrm{P}<0,001)$. Tuttavia, tra i pazienti che hanno ricevuto un'alta dose di mantenimento di aspirina, il rischio era maggiore del $45 \%$ con ticagrelor rispetto a clopidogrel, mentre tra i pazienti che avevano ricevuto una bassa dose di mantenimento, ticagrelor era associato a un rischio inferiore del $21 \%$ ( $\mathrm{P}=0,0006$ per l'interazione). Sebbene la validità di questa osservazione sia ancora contestata (è emersa da analisi di sottogruppi e manca di plausibilità biologica evidente), la FDA ha emesso un avvertimento che una dose di mantenimento superiore a $100 \mathrm{mg}$ di aspirina riduce l'efficacia di ticagrelor e deve essere evitata.

\section{Step 6: Il trial è sufficientemente grande da essere convincente?}

Quando a raggiungere la significatività statistica per l'outcome primario è un trial di piccole dimensioni, la prudenza nell'interpretazione del risultato è d'obbligo, in quanto possono subentrare false positività. 


\section{Esempio}

In uno studio sulla $\mathrm{N}$-acetilcisteina rispetto al placebo per prevenire la nefropatia indotta da agenti di radiocontrasto, ${ }^{20} 1 \mathrm{su} 41$ pazienti trattati con $\mathrm{N}$ acetilcisteina ha avuto un evento di outcome primario (danno renale), rispetto a 9 su 42 pazienti che stavano assumendo placebo, con conseguente rischio relativo con $\mathrm{N}$-acetilcisteina di 0,10 (IC al $95 \%$, da 0,02 a 0,$90 ; \mathrm{P}=0,01)$. Sulla base di questo piccolo studio, la conclusione dello studio che la Nacetilcisteina è un mezzo efficace per prevenire il danno renale è troppo forte; un'affermazione più appropriata è che la $\mathrm{N}$-acetilcisteina potrebbe essere efficace. Una conclusione del genere motiverebbe lo svolgimento di uno studio più ampio e definitivo. Sfortunatamente, una successiva meta-analisi ${ }^{21}$ di 10 studi randomizzati (1916 pazienti) ha riportato che l'evidenza era troppo debole ed eterogenea per supportare l'uso di N-acetilcisteina per questa indicazione.

\section{Step 7: Il trial è stato interrotto troppo presto?}

Capita a volte che uno studio venga interrotto precocemente, perché l'analisi ad interim evidenzia una forte superiorità di un trattamento rispetto ad un altro. Purtroppo questa pratica porta spesso a sopravvalutare l'efficacia di un trattamento, oltre a rischiare di far perdere dati preziosi sugli endpoint secondari e di safety.

\section{Esempio}

Un esempio recente è lo studio SPRINT $^{22}$ sul controllo intensivo rispetto allo standard della pressione arteriosa, che aveva un outcome primario composito di infarto miocardico, sindrome coronarica acuta, ictus, insufficienza cardiaca, morte cardiovascolare.

Lo studio è stato interrotto con una mediana di 3,26 anni anziché al previsto follow-up di 5 anni e, al momento in cui è stato interrotto, il rapporto di rischio per l'outcome primario con controllo intensivo era di 0,75 (IC al 95\%, 0,64 a 0,89; $\mathrm{P}<0,001)$. L'eccezionale velocità di pubblicazione è stata sorprendente; sono trascorse solo 4 settimane tra il momento in cui il trial è stato interrotto e il momento in cui il manoscritto è stato presentato per la pubblicazione. La qualità e la completezza di qualsiasi database provvisorio sono inevitabilmente imperfette - ci saranno eventi primari (e altri) in sospeso ancora da accertare e giudicare. Inoltre, il momento in cui viene interrotta una sperimentazione è il momento in cui è più probabile che sia presente una stima esagerata dell'efficacia. La chiusura ordinata del processo dopo l'interruzione anticipata richiede diversi mesi ed è necessaria per ottenere una solida interpretazione di tutte le prove. La segnalazione anticipata di risultati preliminari e incompleti è generalmente poco saggia.

\section{Step 8: Le preoccupazioni relative alla safety controbilanciano i risultati positivi sull'efficacia?}

Di fronte ad un farmaco nuovo con un'efficacia maggiore dei precedenti, bisogna immediatamente andare a vedere i risultati di safety. Benefici e rischi assoluti vanno presentati in termini di differenze percentuali. Di utilità sarebbe anche avere a disposizione il NNT (number needed to treat) e il NNH (number needed to harm).

La considerazione del numero di pazienti necessario per il beneficio rispetto al numero necessario per danneggiare può fornire una guida al beneficio clinico netto.

11 beneficio clinico netto di un nuovo trattamento può essere specifico per il paziente. Il bilancio per i singoli pazienti tra efficacia e sicurezza non è semplice e possono essere utili tecniche statistiche.

\section{Esempio}

Nello studio SPRINT, ${ }^{23}$ 1'abbassamento intensivo della pressione arteriosa ha portato a un tasso di esito cardiovascolare composito primario inferiore di 1,6 punti percentuali e un tasso di morte inferiore di 1,2 punti percentuali rispetto ai tassi con controllo standard della pressione arteriosa durante un periodo di follow-up mediano di 3,26 anni. Tuttavia, questi benefici devono essere valutati rispetto ai tassi di ipotensione, sincope e danno renale acuto, che erano più elevati (rispettivamente di 1,4 punti percentuali, 1,1 punti percentuali e 1,8 punti percentuali) con un controllo intensivo della pressione sanguigna.

Si noti che tutti questi benefici e rischi, sebbene statisticamente significativi, rappresentano piccole differenze assolute. Pertanto, i comitati di orientamento, i medici curanti e i pazienti devono affrontare una sfida quando cercano di determinare quale strategia adottare.

\section{Step 9: Il bilancio efficacia-sicurezza è paziente-specifico?}

Il bilancio clinico netto di un nuovo trattamento potrebbe risultare declinato in maniera diversa a seconda delle categorie di pazienti (cioè vantaggioso per alcuni, ma deleterio per i pazienti ad aumentato rischio di eventi avversi). Fare un bilancio rischibenefici individualizzato per il singolo paziente però non è facilissimo, anche se di certo rappresenterebbe un importante passo verso una terapia realmente su misura. 


\section{Esempio}

Per lo studio DAPT, ${ }^{24}$ sono stati sviluppati 34 modelli multivariabili per prevedere il rischio di infarto del miocardio o trombosi dello stent e il rischio di sanguinamento maggiore.

Oltre a tenere conto della durata del trattamento antipiastrinico (12 mesi contro 30 mesi), questi modelli includevano 9 caratteristiche del paziente e procedurali e sono stati progettati per consentire la determinazione dei rischi relativi di ischemia rispetto al sanguinamento nei singoli pazienti. Le limitazioni includevano l'omissione di alcune variabili, che sono note per prevedere il rischio di ischemia e sanguinamento, l'incapacità di considerare direttamente i predittori di mortalità e l'assenza di validazione esterna. Questa analisi rappresenta uno sviluppo utile verso l'individuazione dell'assistenza al paziente.

\section{Step 10: Ci sono errori nel disegno o nell'esecuzione del trial?}

Un risultato altamente significativo nell'outcome primario già rassicura molto sul fatto che quel risultato non può dipendere dal caso. Tuttavia bisogna anche andare a vedere che non vi siano bias di disegno o di conduzione dello studio, prima di accettare che il trattamento testato apporti un reale beneficio.

\section{Esempio}

Ad esempio, il primo studio randomizzato di denervazione renale nell'ipertensione resistente al trattamento, SYMPLICITY HTN-2,3725 ha mostrato che a 6 mesi la pressione sistolica era notevolmente più bassa nel gruppo di trattamento rispetto al gruppo di controllo (differenza media, $31 \mathrm{~mm} \mathrm{Hg} ; \mathrm{P}<0,0001$ ). Tuttavia, l'assenza di cecità ha introdotto importanti problemi (ad esempio, effetti placebo e Hawthorne e l'ascertainment bias o detection bias. Nel successivo studio controllato, la denervazione renale SYMPLICITY HTN-3,3926 sembrava essere inefficace, sottolineando in tal modo la potenziale inaffidabilità di studi non controllati.

Un altro punto molto importante da tener presente è il grado di aderenza al trattamento dei soggetti arruolati nello studio oltre al tasso dei drop-out che può andare ad inficiare la validità dello studio.

Lo studio ATLAS ACS 2-TIMI 51 trial 40 di rivaroxaban ${ }^{27}$ rispetto al placebo in pazienti con sindromi coronariche acute ha mostrato differenze tra $\mathrm{i}$ gruppi molto significative a favore della dose più bassa di rivaroxaban rispetto sia all'outcome primario (morte cardiovascolare, infarto del miocardio, o ictus) e morte cardiovascolare da sola. Ma il 27,6\% dei pazienti ha interrotto il trattamento prematuramente e i dati sullo stato vitale mancavano per il $7,2 \%$ dei pazienti, fattori che hanno introdotto incertezza. Questi problemi sembravano essere maggiori in questo studio rispetto ad altri grandi studi che affrontano le sindromi coronariche acute e hanno contribuito alla decisione della FDA di negare l'approvazione di rivaroxaban per questa indicazione. ${ }^{28}$

\section{Step 11: I risultati dello studio sono applicabili ai miei pazienti?}

I risultati di qualsiasi trial, a voler essere rigorosi, sono applicabili solo alla tipologia di pazienti arruolata per quel determinato studio. La questione se sia possibile estrapolare questi risultati ad altri pazienti non è banale.

Altra considerazione importante da fare è la geografia dello studio, cioè le popolazioni studiate. Nel caso dei trial internazionali comprendenti diversi Paesi nei vari continenti, questo è un punto a favore della generalizzabilità dei risultati. Per ragioni opposte, vanno presi con grande attenzione $\mathrm{i}$ risultati di studi monocentrici, che raramente possono avere un impatto sulle linee guida e che spesso mancano di controlli di qualità, a meno che non fungano da studio pilota con risultati confermati da successivi studi con arruolamento multicentrico. Infine, le differenze genetiche, anatomiche, ambientali e dietetiche tra i popoli a volte rendono i risultati difficili da generalizzare in tutti i paesi.

\section{Esempio}

Nel caso del già citato studio SPRINT (che ha valutato i risultati di un trattamento antipertensivo intensivo versus quello standard relativamente ad un endpoint primario composito comprendente infarto, sindromi coronariche acute, ictus, scompenso cardiaco e mortalità cardiovascolare), ad esempio, erano stato esclusi tutti i pazienti sotto i 50 anni d'età e quelli con diabete o con una storia di ictus. Dunque, a voler essere precisi, i risultati di questo studio riguardano solo il $20 \%$ dei pazienti ipertesi.

\section{Messaggi chiave}

1. Prima di trasferire nella pratica corrente i risultati di un nuovo studio clinico, ne va attentamente valutata la significatività statistica.

2. La significatività statistica prescinde dalla significatività clinica ed è intimamente correlata ai concetti di potenza dello studio ed intervallo di confidenza.

3. La valutazione della significatività clinica di uno studio, che ha raggiunto la significatività statistica, prevede un percorso di valutazione di più step.

La Figura 1 sintetizza il corretto percorso di valutazione della significatività di uno studio clinico, prima dell'introduzione del cambiamento di comportamento. 


\section{Studio statisticamente significativo, ovvero Outcome primario POSITIVO}

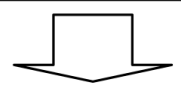

\section{Valutazione degli 11 quesiti base:}

1. Il valore della $P$ fornisce un'evidenza abbastanza forte?

2. Quanto è grande il beneficio del trattamento?

3. L'outcome primario è clinicamente rilevante e consistente?

4. Gli outcome secondari sono di supporto?

5. I risultati dello studio sono confermati per tutti i sottogruppi importanti?

6. Il trial è sufficientemente grande, da essere convincente?

7. Il trial è stato interrotto troppo presto?

8. Le preoccupazioni relative alla safety controbilanciano i risultati positivi sull'efficacia?

9. Il bilancio efficacia-sicurezza è paziente-specifico?

10. Ci sono errori nel disegno o nell' esecuzione del trial?

11. I risultati di questo studio sono applicabili ai miei pazienti?

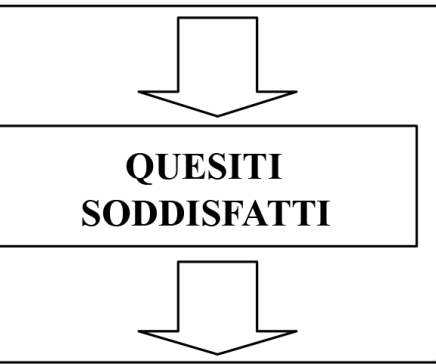

Approvazione normativa, studio sull'efficacia nel mondo reale, analisi del rapporto costo-efficacia, stima del rimborso, orientamento del medico e raccomandazione per l'uso nei pazienti perseguiti

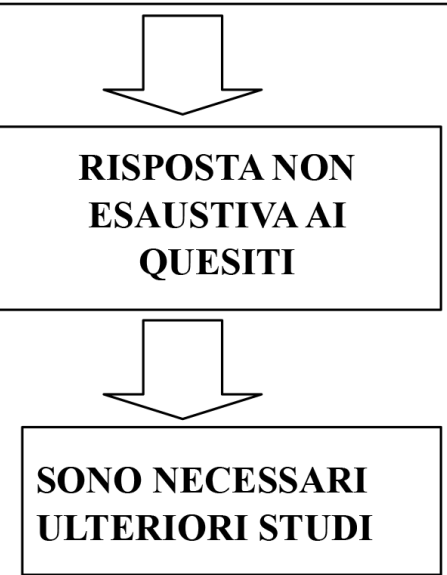

Figura 1. Percorso di valutazione della significatività di uno studio clinic, prima dell'introduzione del cambiamento di comportamento.

\section{Bibliografia}

1. Da Hatch S. Uncertainty in medicine, Editorials, BMJ 2017;357:j2180.

2. Goss PE, Ingle JN, Pritchard KI, et al. Extending aromatase-inhibitor adjuvant therapy to 10 years. N Eng1 J Med 2016;357:209-19.

3. Prasad V, Vandross A, Toomey C, et al. A decade of reversal: an analysis of 146 contradicted medical practices. Mayo Clin Proc 2013;357:790-8.

4. Zanolla L, Graziani MS. Come interpretare correttamente il valore di P?. Biochimica clinica, 2016, vol. 40 , n. 1 p. 40-44.
5. Pocock SJ, Stone GW. The Primary Outcome Is Positive - Is That Good Enough? N Engl J Med 2016; 375:971979.

6. Poisson J, Fedoriw Y, Henderson MP, et al. Performance evaluation of the Helena V8 capillary electrophoresis system. Clin Biochem 2012;45:697-9.

7. McMurray JJV, Packer M, Desai AS, et al. Angiotensinneprilysin inhibition versus enalapril in heart failure. $\mathrm{N}$ Engl J Med 2014;371:993-1004.

8. Lees KR, Zivin JA, Ashwood T, et al. NXY-059 for acute ischemic stroke. N Engl J Med 2006;354: 588-600.

9. Cannon CP, Blazing MA, Giugliano RP, et al. Ezetimibe 
added to statin therapy after acute coronary syndromes. N Engl J Med 2015;372:2387-239.

10. The Action to Control Cardiovascular Risk in Diabetes Study Group. Effects of intensive glucose lowering in type 2 diabetes. N Engl J Med 2008;358:2545-2559.

11. Greene HL, Roden DM, Katz RJ, Woosley RL, Salerno DM, Henthorn RW. The Cardiac Arrhythmia Suppression Trial: first CAST then CAST-II. J Am Coll Cardiol. 1992 Apr;19(5):894.

12. Fox KAA, Poole-Wilson PA, Henderson RA, et al. Interventional versus conservative treatment for patients with unstable angina or non-ST-elevation myocardial infarction: the British Heart Foundation RITA 3 randomised trial. Lancet 2002;360:743-751.

13. Bavry AA, Kumbhani DJ, Rassi AN, Bhatt DL, Askari AT. Benefit of early invasive therapy in acute coronary syndromes: a meta-analysis of contemporary randomized clinical trials. J Am Coll Cardiol 2006;48: 1319-1325.

14. Fox KA, Poole-Wilson P, Clayton TC et al. 5-year outcome of an interventional strategy in non-STelevation acute coronary syndrome: the British Heart Foundation RITA 3 randomised trial. Lancet. 2005 Sep 10-16;366(9489):914-20.

15. Lees KR, Zivin JA, Ashwood T, et al. NXY-059 for acute ischemic stroke. N Engl J Med 2006;354:588-600.

16. Shuaib A, Lees KR, Lyden P, et al. NXY-059 for the treatment of acute ischemic stroke. N Engl J Med 2007;357:562-57.

17. Zinman B, Wanner C, Lachin JM, et al. Empagliflozin, cardiovascular outcomes, and mortality in type 2 diabetes. N Engl J Med 2015;373:2117-2128.

18. Wallentin L, Becker RC, Budaj A, et al. Ticagrelor versus clopidogrel in patients with acute coronary syndromes. N Engl J Med 2009;361:1045-1057.

19. Carroll KJ, Fleming TR. Statistical evaluation and analysis of regional interactions: the PLATO trial case study. Stat Biopharm Res 2013;5:91-101.

20. Tepel M, van der Giet M, Schwarzfeld C, Laufer U, Liermann D, Zidek W. Prevention of radiographiccontrast-agent-induced reductions in renal function by acetylcysteine. N Engl J Med 2000;343:180-184.

21. Sun Z, Fu Q, Cao L, Jin W, Cheng L, Li Z. Intravenous $\mathrm{N}$-acetylcysteine for prevention of contrast-induced nephropathy: a meta-analysis of randomized, controlled trials. PLoS One 2013;8.

22. Drazen JM, Morrissey S, Campion EW, Jarcho JA. A SPRINT to the finish. N Engl J Med 2015;373:2174-2175.

23. The SPRINT Research Group. A randomized trial of intensive versus standard blood-pressure control. N Engl J Med 2015;373:2103-2116.

24. Mauri L, Kereiakes DJ, Yeh RW, et al. Twelve or 30 months of dual antiplatelet therapy after drug-eluting stents. N Engl J Med 2014;371:2155-216.

25. Esler MD, Böhm M, Sievert H, Rump CL. Catheterbased renal denervation for treatment of patients with treatment-resistant hypertension: 36 month results from the SYMPLICITY HTN-2 randomized clinical trial. Eur Heart J. 2014 Jul;35(26):1752-9.

26. Alexandre Persu, Fadl Elmula M, Fadl Elmula et al. Renal Denervation After Symplicity HTN-3 - Back to Basics. Review of the Evidence.Eur Cardiol. 2014 Dec; 9(2): 110-114.

27. Mega JL, Braunwald E, Wiviott SD, et al. Rivaroxaban in patients with a recent acute coronary syndrome. $\mathrm{N}$ Engl J Med 2012;366:9-19.

28. Krantz MJ, Kaul S. The ATLAS ACS 2-TIMI 51 trial and the burden of missing data: (Anti-Xa Therapy to Lower Cardiovascular Events in Addition to Standard Therapy in Subjects With Acute Coronary Syndrome ACS 2-Thrombolysis In Myocardial Infarction 51). J Am Coll Cardiol 2013 Aug 27;62(9):777-81. 


\title{
Dalla medicina personalizzata alla medicina di precisione
}

\author{
Gianfranco Gensini, ${ }^{1}$ Nicoletta Scarpa ${ }^{2}$ \\ ${ }^{1}$ Direttore Scientifico Multimedica IRCCS; ${ }^{2} \mathrm{CESMAV}$, Firenze, Italia
}

\section{Introduzione}

Per medicina di precisione si intende il tentativo di personalizzare il più possibile prevenzione, diagnosi e cure in base alle caratteristiche del singolo (persona priva di elementi di rischio, persona con elementi di rischio, paziente con eventi clinici, paziente in riabilitazione dopo eventi clinici e/o in prevenzione nei confronti di possibili eventi futuri). ${ }^{1,2}$ Negli ultimi due decenni, stiamo assistendo a un cambiamento epocale: si sta affermando un concetto di medicina cucito sulle differenze individuali, che tiene conto delle differenze genetiche, dell'ambiente, delle caratteristiche del microbioma e dello stile di vita delle singole persone.

Non è raro sentire utilizzare il termine medicina personalizzata come sinonimo di medicina di precisione. Ma l'uso indiscriminato dei due termini non appare appropriato, in quanto la medicina fino dai suoi albori ha sempre ricercato nelle caratteristiche della persona gli elementi utili per adattare al meglio gli interventi preventivi, di cura e di riabilitazione, è sempre stata personalizzata o almeno si è sempre cercato di ragionare in funzione del singolo paziente e delle sue caratteristiche individuali.

Per comprendere il senso di questa affermazione è sufficiente pensare a quanto veniva affermato da Ippocrate circa 2.500 anni fa. Già agli albori della medicina infatti Ippocrate suggeriva di somministrare diverse medicine a pazienti diversi: quelle dolci non danno lo stesso beneficio a tutti, né gli astringenti producono lo stesso effetto, né i pazienti sono in grado di assumere la stessa bevanda. A fine Ottocento il concetto di individualità viene rafforzato anche dal

Corrispondente: Gianfranco Gensini, MultiMedica Spa, Via Fantoli 16/15, Milano, Italia.

E-mail: gfgensini@gmail.com

Articolo pubblicato secondo la Creative Commons Attribution NonCommercial 4.0 License (CC BY-NC 4.0).

${ }^{\circ}$ Copyright: the Author(s), 2020

Licensee PAGEPress, Italy

QUADERNI - Italian Journal of Medicine 2020; 8(1):58-61 medico William Osler, considerato il padre della medicina moderna, il quale dichiarava in un'affermazione citata assai di frequente che $a$ volte $\grave{e}$ molto più importante sapere che tipo di paziente ha una malattia piuttosto che quale tipo di malattia ha un paziente.

Se quindi la medicina è sempre stata orientata verso la personalizzazione, in che cosa consiste il cambiamento epocale che stiamo vivendo? E siamo davvero sicuri che il concetto di medicina di precisione sia un concetto nuovo?

Cercheremo di ripercorrere le tappe principali che hanno portato alla medicina di precisione e di capire quali sono i possibili sviluppi di questa nuova medicina.

\section{Le tappe storiche}

Come anticipato si è sempre saputo o almeno si è sempre percepito che i pazienti non fossero tutti uguali e che quindi la terapia dovesse essere personalizzata sul singolo paziente.

È all'inizio del 1900 però che con l'identificazione dei gruppi sanguigni si ha la prima prova tangibile che i pazienti non sono uguali. ${ }^{3}$ Grazie alla tecnologia dell'epoca è stato possibile per la prima volta identificare il gruppo sanguigno e assumere nella cura un approccio moderno, preciso. Le trasfusioni diventano quindi il primo esempio concreto di medicina di precisione. ${ }^{3}$

Successivamente, l'idea della variabilità umana viene rafforzata dalla risposta anomala ad alcuni trattamenti farmacologici, risposta che era possibile registrare solo in certe persone.

Per esempio, negli anni Cinquanta il medico tedesco Vogel scoprì che non tutti sono in grado di percepire il gusto della feniltiocarbammide. Sempre negli stessi anni la farmacogenomica mette chiaramente in luce che le caratteristiche genetiche possono determinare risposte diverse ai farmaci. ${ }^{4}$ Per esempio è stato scoperto in quegli anni che una carenza genetica individuale dell'enzima glucosio-6fosfato-deidrogenasi a livello dei globuli rossi, relativamente diffusa in pazienti neri e rara nei soggetti caucasici, induce anemia emolitica in seguito 
a trattamento con la primachina, un farmaco antimalarico. ${ }^{4}$

Sempre grazie alla farmacogenomica si è visto che bassi livelli o varianti di un altro enzima, la pseudocolinesterasi, sono risultati responsabili di risposte anomale alla succinilcolina, un agente impiegato nell'induzione dell'anestesia. ${ }^{4}$

Già nel corso del 900 l'approccio culturale della medicina e la visione era quella di personalizzare e di intervenire in modo preciso sul singolo paziente. Mancavano però le conoscenze e gli strumenti tecnologici per rendere concreta questa visione.

È solo nel 2000 che grazie al sequenziamento del genoma umano (2001) e via via allo sviluppo delle nuove scienze omiche che assistiamo a una vera rivoluzione della medicina di precisione. L'avvento di metodi per il sequenziamento del DNA ha cambiato profondamente la nostra comprensione dei meccanismi alla base della vita. È stato possibile identificare e diagnosticare malattie ereditarie, studiare le componenti genetiche delle malattie comuni multifattoriali, comprendere le alterazioni genomiche dei tumori, sviluppare nuovi trattamenti farmacologici e predirne efficacia ed effetti avversi.

Il consenso scientifico internazionale definisce la Medicina di precisione come lo studio delle caratteristiche genotipiche e fenotipiche (ambiente, stile di vita) di ogni singolo individuo, e si pone lo scopo di integrare tra loro tali informazioni, conferendo caratteristiche uniche a patologie complesse che possono cosi essere diagnosticate $e$ curate in maniera più efficiente, con un chiaro impatto sia dal punto di vista medico che socio-economico. ${ }^{1}$

Secondo i National Institutes of Health, applicare tali approcci alla medicina potrebbe estendere le possibilità di trattamento anche a molte patologie ancora intrattabili, di cui non è stato ancora individuato un marcatore bersaglio da colpire in modo specifico con un farmaco. L'obiettivo principale è quindi quello di combinare informazioni genetiche e dati clinici per personalizzare le cure, a seconda delle caratteristiche del paziente. Alla base c'è il presupposto che, confrontando i dati genetici delle persone con le conoscenze a disposizione sulle diverse malattie, sarà possibile ottenere diagnosi più accurate e terapie più efficaci. ${ }^{5}$

$\mathrm{Si}$ può quindi dire che sebbene l'approccio personale e la relazione medico-paziente siano elementi fondamentali della medicina di precisione non sono la novità. Il presupposto indispensabile per lo sviluppo della medicina di precisione è quindi l'esplosione dei dati legati agli studi di biologia molecolare, il sequenziamento del genoma umano, oltre a discipline scientifiche, come epigenetica, transcrittomica, proteomica e metabolomica che hanno prodotto una vasta quantità di informazioni che permettono una sempre più precisa caratterizzazione del paziente.

\section{Il 2015 anno della svolta}

La medicina di precisione diventa quindi molto popolare negli ultimi anni anche per le prospettive politico-sanitarie che vengono attribuite.

Il 2015 è un anno importante perché grazie a Barack Obama allora presidente degli Stati Uniti viene lanciato il progetto Precision Medicine per cui vengono stanziati 215 milioni di dollari. Nell'arco di poco tempo si è quindi passati da un annuncio di visione all'implementazione di azioni di sostegno.

La medicina di precisione diventa quindi la medicina del futuro, e viene definita come la medicina delle $4 \mathrm{P}: 6,7$ i) personalizzata, in quanto ciascun individuo ha caratteristiche proprie, ciascuno è geneticamente unico non saranno quindi più considerati rappresentativi popolazioni standard; ii) preventiva e predittiva in quanto capace di individuare le patologie a rischio; iii) partecipativa, in quanto si ritiene che ogni individuo può avere un ruolo decisivo nella gestione della propria patologia se acquisisce consapevolezza della propria storia medica e viene coinvolto nelle scelte terapeutiche.

Ciò che si sta verificando è quindi un cambiamento radicale se si pensa che tradizionalmente la medicina curava una malattia alla comparsa delle manifestazioni cliniche, il passo successivo è stato quello di concentrarsi sui fattori di rischio e la loro prevenzione. Per esempio applicare una politica antifumo per ridurre il rischio di cancro del polmone. Ora siamo di fronte a una svolta ulteriore in quanto grazie alla tecnologia non solo possiamo analizzare il nostro genoma ma possiamo anche capire la risposta del nostro genoma all'ambiente (epigenetica). ${ }^{6}$ È possibile quindi capire la predisposizione alle malattie ma anche la capacità di risposta alle terapie quindi l'efficacia e il rischio di effetti collaterali (per esempio allergie).

\section{Dalla Medicina Basata sulle Evidenze alla Medicina di Precisione}

Sono trascorsi 24 anni da quando fu pubblicato sulla prestigiosa rivista scientifica Journal of the American Medical Association il primo articolo (1992) che proponeva alla comunità scientifica internazionale un nuovo approccio strutturato alla medicina, l'Evidence Based Medicine (EBM). ${ }^{8}$ In questi anni l'EBM ha svolto e continua a svolgere un ruolo fondamentale nella ricerca clinica, ponendo al centro della medicina le evidenze, o prove di efficacia, degli atti sanitari (uso di farmaci, interventi, modalità organizzative della sanità) utilizzando essenzialmente 
tre elementi: i) la competenza e l'esperienza del clinico; ii) l'analisi dei risultati delle migliori ricerche cliniche; e iii) i valori del paziente. Ciò nonostante oggi ci si rende conto che questo modus operandi per quanto razionale e analitico, non è più sufficiente per garantire un approccio adeguato alla gestione clinica dei pazienti complessi che caratterizzano larga parte della popolazione attuale. Il problema nasce in particolare dall'aumento della longevità della popolazione, aspetto che determina con frequenza crescente la presenza e la sovrapposizione di più patologie. La medicina contemporanea si trova quindi di fronte sempre più spesso pazienti complessi in cui le comorbilità o le multimorbilità, richiedono di essere affrontati da team multidisciplinari. Allo stesso tempo, il progresso scientifico dovuto allo sviluppo delle nuove tecnologie, così come l'accrescersi delle conoscenze, dalla genomica alla transcrittomica, all'epigenetica alla proteomica, alla metabolomica, e a tutte le medomiche richiedono nuove competenze e nuovi criteri di indagine. Oggi, la medicina e quindi l'approccio clinico basato sull'EBM, deve dotarsi non solo di nuove metodologie di ricerca clinica e di nuovi strumenti di diagnosi, ma anche di un nuovo modello formativo rivolto alla classe medica finalizzato a ottenere risorse umane, ossia clinici e scienziati con un expertise multi fattoriale.

Il primo passo da compiere è dunque capire quali strumenti e o servizi possono aiutarci a cambiare il nostro attuale approccio clinico basato sull'EBM. Il problema essenziale della medicina è oggi la condivisione di informazioni legate alla complessità. Questa esigenza, vivacemente percepita in ambito accademico, è oggi elemento di attenzione e riferimento anche per i governi impegnati a riscrivere sistemi di welfare sanitario sostenibili e quindi fondati sulla predittività e sulla prevenzione non più in termini di popolazione ma arrivando sempre di più al singolo individuo. In questo senso negli Stati Uniti d'America lo stesso presidente, Barack Obama, nel suo Discorso sullo Stato dell'Unione il 20 gennaio 2015 lanciò la sfida verso una medicina della precisione che consentisse di arrivare il più presto possibile a un approccio personalizzato della medicina. Questa iniziativa, subito ripresa dal New England Journal of Medicine e da un ampio numero di autorevolissime riviste scientifiche, si è progressivamente affermata come un obiettivo fondamentale della medicina moderna, attuale e futura. La medicina della precisione è riassumibile dal motto: data driven treatments as unique as your own body, una prospettiva in grado di arrivare al giusto farmaco alla giusta dose al paziente giusto (the right drug at the right dose to the right patient), ivi comprese le differenze di genere. ${ }^{9}$

La precision medicine, è già molto avanti in ambiti di ricerca come l'oncologia dove la ricerca di biomarker specifici sta dando grandi risultati, una frontiera nel quale anche il cardiovascolare sia in termini di popolazione che di diagnostica sta producendo dati molto interessanti e significativi. ${ }^{10,11}$

Se la precision medicine rappresenta il futuro dietro l'angolo della ricerca scientifica più innovativa, riteniamo che la gestione della complessità debba necessariamente passare attraverso un modello di EBM 2.0 che contempli, ma soprattutto integri vari approcci clinici, dalla Precision Medicine, alla Knowledge Based Medicine al System Medicine e last but not least alla Narrative Medicine secondo una visione realmente olistica del paziente in grado di analizzare e condividere nella relazione medico paziente gli aspetti psicologici a quelli sociali, fino al credo religioso, che incidono in modo definito nel decorso delle varie patologie.

Recentemente la medicina di precisione ha visto la comparsa di una nuova opportunità di trattamento delle neoplasie attraverso la cosiddetta terapia agnostica, che non segue il classico modello istologico, secondo una sequenza che prevede: i) la localizzazione del tumore; ii) la tipizzazione istologica; iii) l'eventuale presenza di biomarkere/o mutazioni genomiche; iv) l'identificazione di possibili farmaci a bersaglio molecolare.

Nel modello di terapia agnostica la sede del tumore non è più un elemento diagnostico fondamentale, mentre $\mathrm{i}$ test genetici diventano fondamentali per decidere quale trattamento indipendentemente dalla sede. ${ }^{12}$

\section{Conclusioni}

In conclusione, questa visione globale e innovativa di un EBM 2.0 omnicomprensiva, che è stata anche definita Medicina del Rinascimento si fonda su alcuni elementi fondamentali che possiamo così sintetizzare: i) attuare la medicina della precisione attraverso un network integrato di competenze scientifiche multidisciplinari in rete; ii) utilizzare e integrare le conoscenze scientifiche grazie a un uso coerente delle tecnologie più innovative e dell'informatica, attraverso la systems medicine; iii) implementare la relazione medico-paziente, che deve essere sempre più attenta non solo all'anamnesi della storia del paziente, ma a tutti quegli aspetti più intimi della psiche umana fino a coglierne le diversità inerenti il credo religioso; iv) creare un curriculum formativo universitario standardizzato per i futuri medici.

\section{Bibliografia}

1. National Research Council (US). Committee on a framework for developing a new taxonomy of disease. National Research Council 2011. 
2. Ginsburg G, Phillips KA. Precision medicine: from science to value. Health Aff (Millwood) 2018;37:694701

3. Erb IH. Blood group classification. The Can Med Association J 1940. https://www.ncbi.nlm.nih.gov/pmc/ articles/PMC537907/pdf/canmedaj00217-0006.pdf

4. Paoletti V, Parlapiano C, et al. La farmacogenetica: aspetti teorici e pratici. Ann Ist Super Sanità 1983;23:241-56

5. National Institute of Health. What is precision medicine initiative? National Institute of Health. https://ghr.nlm. nih. gov/primer/precisionmedicine/initiative

6. Cappelletti P. Medicina di precisione e medicina di laboratorio. La Rivista Italiana della Medicina di Laboratorio - Italian Journal of Laboratory Medicine 2016;12:129-33.

7. Alonso SG, de la Torre DI, et al. Predictive, Personalized, Preventive and Participatory (4P)
Medicine Applied to Telemedicine and eHealth in the Literature. J Med Syst 2019;43:140.

8. Evidence Based Medicine Working Group. Evidencebased medicine. A new approach to teaching the practice of medicine. JAMA 1992;268:2420-5.

9. President Barack Obama. The precision medicine iniziative: data driven treatments as unique as your own body. The White House 2015. https://obamawhitehouse. archi ves.gov/blog/2015/01/30/precision-medicine-initiativedata-driven-treatments-unique-your-own-body

10. Turnbull AK. Personalized medicine in cancer: where are we today? Fut Oncol 2015;20.

11. Leopold JA, Loscalzo J. The emerging role of precision medicine in cardiovascular disease. Circ Res 2018;122: 1302-15.

12. Flaherty KT, Dung $\mathrm{T}$, et al. Tissue agnostic drug development. ASCO Educational book 2018. https:// ascopubs.org/doi/pdf/10.1200/EDBK 173855 


\title{
Una possibile alternativa alle decisioni: il metodo Clinical Multi-criteria Decision Assessment (CMDA)
}

\author{
Ombretta Para \\ Medicina Interna 1, AOU Careggi, Firenze, Italia
}

Per la valutazione globale dell'impatto di un trattamento farmacologico, nel tentativo di utilizzare un approccio olistico, dovrebbero essere presi in considerazione diversi parametri clinici, organizzativi ed economici, confrontando e ponderando più criteri.

$\mathrm{Si}$ rende pertanto necessario un cambiamento metodologico: per scegliere il trattamento farmacologico più adatto per un determinato quadro clinico dobbiamo passare da un contesto decisionale basato sulla valutazione di un singolo criterio ad un quadro più completo e sistematico che consenta ai decisori di valutare contemporaneamente tutti i molteplici parametri.

Da questa visione nasce un modello metodologico basato su criteri multipli chiamato Valutazione Decisionale Clinica Multicriterio (Clinical Model Decision Assesment - CMDA) utilizzato per ottimizzare il processo decisionale clinico che porta alla scelta del miglior trattamento farmaceutico (o dispositivo medico) per la patologia specifica.

L'utilizzo di tale modello è in grado di fornire un'indicazione chiara e completa del miglior trattamento da intraprendere, rivestendo un ruolo decisivo nella scelta terapeutica del medico.

In ambito di ricerca clinica il gold standard è sicuramente lo studio randomizzato controllato (RCT). Gli RCT vengono impiegati per valutare l'effetto di un intervento in cui i pazienti vengono assegnati in modo casuale, in cieco, a ricevere $i$ trattamenti che vengono confrontati. Tale metodo permette di eliminare bias di selezione ed i possibili pregiudizi del medico rispetto al trattamento, consentendo di utilizzare la teoria della probabilità. ${ }^{1}$

Negli studi clinici gli endpoint primari e secondari

Corrispondente: Ombretta Para, Medicina Interna 1, AOU Careggi, Firenze, Italia.

E-mail: ombretta.para@gmail.com

Articolo pubblicato secondo la Creative Commons Attribution NonCommercial 4.0 License (CC BY-NC 4.0).

${ }^{\circ}$ Copyright: the Author(s), 2020

Licensee PAGEPress, Italy

QUADERNI - Italian Journal of Medicine 2020; 8(1):62-66 dovrebbero indicare quale trattamento sia più efficace per una determinata patologia in una specifica popolazione di pazienti; pertanto in un RCT la corretta formulazione dell'endopoint primario è un punto estremamente critico ed importante che è necessario considerare come primo passo nella progettazione dello studio.

Visto il rigore metodologico con cui viene costruito un RCT, esso è in grado di fornire i risultati più attendibili dal punto di vista scientifico riguardo l'efficacia di un trattamento rispetto ad un altro.

Tuttavia anche gli RCT presentano degli svantaggi: ${ }^{2,3}$ possono essere costosi e richiedere molto tempo per l'esecuzione e l'esecuzione. Inoltre la popolazione esaminata potrebbe non essere realmente rappresentativa della popolazione generale.

Poiché la dimensione del campione e il potere statistico di un RCT si basano sull'endpoint primario selezionato, né gli RCT né le meta-analisi sono in grado di offrire contemporaneamente prove su tutti i risultati importanti necessari al fine di stabilire l'efficacia clinica relativa complessiva per ciascun trattamento.

Raramente è possibile valutare in modo completo l'efficacia di un intervento usando semplicemente $\mathrm{i}$ dati di un'unico studio clinico per cui è necessario un metodo analitico in grado di acquisire dati da una serie diverse di fonti (RCT, studi osservazionali, ecc.).

Inoltre, anche per una corretta analisi costibenefici è necessario considerare più parametri clinici, la conformità e la via di somministrazione, l'efficacia a lungo termine e tutti gli aspetti della sicurezza e dei costi.

Un nuovo strumento utilizzato per confrontare più interventi è la meta-analisi a rete, un'estensione della meta-analisi tradizionale ${ }^{4,5}$ che attraverso un'analisi probabilistica ${ }^{6}$ consentendo di classificare i trattamenti in base alla loro probabilità di essere i trattamenti di maggior efficacia.

La metanalisi a rete (o network meta-analysis NETMA), pur esistendo già da diverso tempo, ha trovato solo recentemente una collocazione riconosciuta nel campo della evidence-based medicine. Dal punto di vista teorico, la NETMA è uno strumento sviluppato per operare nei casi in cui si 
realizzano ambedue le seguenti condizioni: i) confrontare due trattamenti $\mathrm{A}$ e $\mathrm{B}$ in cui non esiste uno studio controllato che abbia confrontato A $v s$ B l'uno contro l'altro (ossia non esiste un trial clinico di confronto testa a testa o diretto tra A e B); ii) esistono invece alcuni studi controllati nei quali un terzo trattamento (es. C) è stato confrontato (in studi separati) sia con A che con B. ${ }^{4-7}$

Un ulteriore metodo comunemente usato per includere più parametri nella valutazione è la stima della qualità della vita intesa come anno di vita adeguato alla qualità o QALY. ${ }^{7}$ Il QALY (acronimo di Quality Adjusted Life Years) è un'unità di misura che valuta più criteri, combinando insieme la durata della vita con la qualità della stessa. Purtroppo però gli studi clinici e osservazionali non esprimono $i$ risultati in termini di anno di QALY. ${ }^{8}$

Queste considerazioni chiariscono perché sia fondamentale creare un modello decisionale alternativo che prenda in considerazione più criteri in grado di bilanciare tutti i possibili endpoint per aiutare il decisore a prendere la scelta giusta.

L'analisi decisionale a criteri multipli (MCDA) è una sotto-disciplina della ricerca in contesti come business, governo e sanità.

I criteri che vengono presi in considerazione nella valutazione e che spesso entrano in conflitto tra loro il costo e la qualità. Per esempio, nell'acquisto di un'auto, i costi, il comfort, la sicurezza e il risparmio di carburante possono essere alcuni dei criteri principali che consideriamo: è inusuale che l'auto più economica sia la più comoda e la più sicura.

In diverse discipline scientifiche e campi sociali, l'analisi decisionale multicriteriale è ben sviluppata, ha ottenuto un buon consenso generale e viene costantemente utilizzata. ${ }^{9-12}$ Esistono solo pochissime applicazioni dell'MCDA nella ricerca sui sistemi sanitari, anche se questa tendenza è in aumento. ${ }^{13-15}$

Tale metodo è particolarmente utile nella risoluzione di problemi caratterizzati dalla necessità di scegliere tra diverse alternative ${ }^{16}$ perché permette di identificare l'importanza relativa di ogni parametro esiste, è un' analisi razionale, coerente e trasparente ed è ragionevolmente facile da usare. ${ }^{17}$

Il primo passo nella MCDA è stilare la lista di tutte le possibili alternative di intervento per risolvere un problema complesso.

Il secondo passo è chiarire o identificare tutti i criteri pertinenti per la valutazione delle alternative disponibili e dovrebbe portare alla produzione di una tabella delle conseguenze contenente criteri (parte sinistra della tabella) e alternative (parte destra della tabella).

Nella terza fase, le conseguenze della scelta di ogni alternativa sono riportate nella cella appropriata. I dati riportati possono essere note o risposte a domande specifiche (sì/no/non lo so) o una scala numerica; in quest'ultimo caso il significato o il valore devono essere chiaramente spiegati.

Il quarto passo è quello di classificare ogni opzione rispetto a ciascun criterio e ciò porterà alla produzione di una serie di gradi in base alla tabella delle conseguenze che esprimono le preferenze del decisore o il riassunto delle valutazioni oggettive e che possono identificare quali opzioni sono preferite dal punto di vista di ciascun criterio selezionato.

Nella quinta fase la tabella delle classifiche può essere utilizzata per eliminare le alternative ritenute secondarie o che non riescono a raggiungere un livello minimo accettabile su uno o più degli obiettivi. Un'alternativa 'A' può essere considerata dominante rispetto all'alternativa ' $B$ ' se: i) l'alternativa $A$ è migliore dell'altra su tutti i criteri; ii) l'alternativa A è migliore dell'altra solo per alcuni criteri e uguale in altri. Nel caso in cui più di un'opzione favorevole emerga da questa procedura, sarà necessario fare una scelta, poiché non ci sarà alcuna opzione che sia la migliore su tutti i criteri. A questo punto dovrebbe essere eseguito un compromesso tra i diversi criteri. Poiché le restanti alternative disponibili saranno ritenute peggiori su alcuni dei criteri e tutti i criteri non hanno la stessa rilevanza, è necessario un metodo per identificare e classificare le prestazioni in base ciascun criterio (questo è il sesto passo).

Esistono diversi modi per farlo. Il metodo più efficace e trasparente può essere la conversione delle conseguenze in punteggi di valore. Ciò consiste nell'effettuare una stima del valore di ciascuna conseguenza partendo da ciascun criterio. Generalmente, viene utilizzata una scala da 100 a 0 , dove 100 (o 10 o 1 ) rappresenta la migliore prestazione e 0 la peggiore; in questo modo vengono chiaramente definite le migliori e peggiori performance. Dopo aver identificato il punteggio migliore e il peggiore, è necessario derivare i punteggi intermedi. È molto facile se abbiamo un criterio misurabile in cui le misure corrispondono ai punteggi di valore. In assenza della misurabilità dei criteri, il DM dovrà valutare direttamente ciascuna alternativa. ${ }^{18}$

L'MCDA può essere applicato anche nel processo di valutazione del miglior trattamento farmaceutico per una specifico patologia in un determinato territorio. L'ipotesi che suggeriamo è di applicare il modello MCDA in ambito clinico al fine di aiutare $\mathrm{i}$ clinici ad ottenere la migliore decisione basata sulla Evidence Based Medicine e su una metodologia trasparente e sistematica che consenta ai clinici di valutare simultaneamente tutti i criteri pertinenti. Il modello MCDA applicato in questo campo potrebbe essere chiamato valutazione decisionale con criteri multipli clinici (CMDA) e potrebbe essere integrato successivamente in un contesto di un più ampio 
quadro MCDA per un'ulteriore valutazione economica o sociale.

Nel CMDA l'approccio innovativo è quello di prendere in considerazione un consenso clinico per la selezione di tutti i criteri clinici (domini) e parametri correlati pertinenti e per l'attribuzione di pesi specifici a ciascuno dominio e parametro di valutazione. Ciò significa che i risultati clinici misurati su un endpoint specifico (parametri) saranno ponderati in base all'importanza che un consenso clinico, composto da un comitato scientifico (a livello regionale o nazionale) darà al dominio specifico. Utilizzando l'approccio CMDA, il consenso clinico diventa il punto cardine della procedura di valutazione che mira a scegliere i migliori trattamenti clinici, garantendo un processo trasparente e oggettivo.

Nella CMDA, i risultati di meta-analisi e studi osservazionali sono utilizzati da un consenso clinico dopo aver attribuito pesi a domini specifici e parametri correlati. La decisione deriverà da un confronto di tutte le conseguenze (vale a dire efficacia, sicurezza, aderenza, via di somministrazione) esistenti dietro la scelta di utilizzare un trattamento farmacologico specifico. ${ }^{18}$

Il CMDA combina risultati di meta-analisi, prove di revisione della letteratura e risultati di studi osservazionali con pesi di consensi clinici su dominio e parametri in modo tale da produrre un punteggio (in valore assoluto) che collega ciascun parametro con un intervento specifico, quindi un punteggio finale per ciascun trattamento. Più alto è il punteggio finale, più appropriato è l'intervento per il trattamento della malattia, considerando tutti i criteri (dominio e parametri). I risultati consentiranno al medico di valutare il miglior trattamento clinico per i suoi pazienti considerando allo stesso tempo tutti i criteri rilevanti come l'efficacia clinica e la via di somministrazione.

Riportiamo un esempio tratto dalla letteratura: ${ }^{18}$ l'ipotesi di applicazione della CMDA ai farmaci utilizzati nell'osteoporosi (OP).

L'osteoporosi è caratterizzata da una ridotta massa ossea e un'interruzione dell'architettura ossea, con conseguente aumento del rischio di fratture da fragilità. L'onere economico delle fratture da fragilità è stato stimato in $€ 37$ miliardi. I costi dovrebbero aumentare del $25 \%$ nel $2025 .{ }^{19}$

L'onere della frattura aumenta con l'invecchiamento della popolazione, specialmente nei paesi occidentali. Pertanto, la prevenzione delle fratture è uno scopo importante per il processo decisionale. Dopo aver corretto i fattori di rischio e lo stile di vita modificabili, è necessario un intervento farmacologico nei pazienti ad alto rischio di frattura affetti da OP. In realtà i bifosfonati sono considerati la terapia di prima linea per la prevenzione e il trattamento delle fratture osteoporotiche, sia vertebrali che non vertebrali. ${ }^{20}$

La riduzione dell'incidenza di nuove fratture vertebrali è l'endpoint più comune e rilevante per ottenere la registrazione di qualsiasi nuovo farmaco per la prevenzione primaria o secondaria dell'OP. La maggior parte degli studi randomizzati controllati (RCT) sulla prevenzione dell'osteoporosi considera la prevenzione delle fratture non vertebrali come endpoint secondario anche se la mortalità e la morbilità delle fratture non vertebrali e specialmente le fratture dell'anca sono più rilevanti rispetto a quelle vertebrali fratture.

Le riduzioni del rischio relativo (RR) per lo sviluppo di nuove fratture vertebrali e non vertebrali variavano rispettivamente dal $41 \%$ al $70 \%$.

Questi dati sono riferiti all'effetto di ogni singolo bisfosfonato rispetto al placebo, ma non sono disponibili dati da RCT di confronto diretti focalizzati sulla riduzione dell'incidenza di nuove fratture vertebrali e non vertebrali poiché le dimensioni del campione di studio e la durata del follow-up dovrebbero essere rispettivamente molto più grandi $\mathrm{e}$ più lunga e i costi conseguenti sarebbero inaccessibili. Un altro metodo comunemente usato per confrontare i bifosfonati si basa sulla valutazione della variazione della BMD: ${ }^{21}$ un aumento della BMD è correlato a una riduzione del rischio di fratture, anche se è solo uno dei fattori determinanti dell'attività anti-fratturativa dei bifosfonati. 22,23

Tuttavia la valutazione dell'efficacia di un trattamento per l'osteoporosi si basa non solo sull'efficacia nella prevenzione delle fratture, ma anche su altri fattori rilevanti come sicurezza, conformità, ${ }^{24}$ efficacia a lungo termine e via di somministrazione, soprattutto in una malattia cronica datata. Valutando diversi fattori un farmaco che si era dimostrato estremamente efficace dal punto di vista clinico in un contesto sperimentale può così diventare meno efficace nella pratica quotidiana se l'aderenza al trattamento o la sua sicurezza a lungo termine risultano scarse o se si verifica una perdita di efficacia nel tempo. D'altra parte, gli strumenti migliori per indagare sulla sicurezza e l'aderenza terapeutica sono i dati di registro e gli studi di coorte.

Inoltre, i dati degli studi randomizzati e delle meta-analisi sia classiche che a rete, nonché gli studi di coorte sono inevitabilmente deboli, poiché analizzano solo un risultato alla volta. Si rende necessaria una valutazione di tutti i parametri e risultati rilevanti per facilitare il processo decisionale basato sull'evidenza.

Abbiamo postulato che un consenso clinico potrebbe accertare come domini: efficacia clinica a 3 anni, aderenza, cambiamento nella BMD, sicurezza e via di somministrazione. Lo stesso consenso deve 
definire tutti i parametri per ciascun dominio e quindi attribuire il peso relativo a ciascuno di essi.

L'uso dell'MCDA si sta diffondendo progressivamente proprio perché sembra il metodo più accurato per affrontare e risolvere situazioni complesse, in grado di considerare i molteplici fattori che influenzano le scelte terapeutiche.

Il CMDA può essere utile anche nel contesto di una valutazione farmaco-economica, anche allo scopo di valutare gli interventi sanitari nazionali o regionali.

Uno dei limiti più rilevanti di questo metodo è la relatività dei domini e dei parametri stabiliti dal consenso clinico che può variare in base alla sua composizione. La variabilità dell'accordo di consenso sul peso dei parametri può causare punteggi finali diversi per gli stessi interventi in diversi paesi. Un altro limite è identificare il valore di ciascun intervento per ciascun criterio o parametro considerando la letteratura. Il modo migliore dovrebbe essere quello di mettere i risultati da studi di confronto diretti o meta-analisi probabilistiche, quando possibile.

In conclusione, sembra possibile applicare la metodologia dell'MCDA in ambito clinico attraverso un nuovo modello modificato, il CMDA. La valutazione delle decisioni elaborata da questo modello potrebbe essere di ausilio per effettuare il miglior trattamento disponibile per una determinata patologia. Saranno tuttavia necessari ulteriori studi applicativi per superare alcuni limiti e migliorare il modello di CMDA. ${ }^{19}$

\section{Bibliografia}

1. Kenneth F, Schulz, David A. Grimes Generation of allocation sequences in randomised trials: chance, not choice THE LANCET February 9, 2002;359:515-19.

2. Sanson-Fisher RW, Bonevski B, Green LW, D'Este C. Limitations of the randomized controlled trial in evaluating population-based health interventions. Am J Prev Med 2007;33(2):155-61.

3. Rothwell PM. External validity of randomised controlled trials: "to whom do the results of this trial apply?". Lancet 2005;365(9453):82-93.

4. Ades AE, Welton N, Lu G. Introduction to Mixed Treatment Comparisons. Bristol, United Kingdom: University of Bristol; 2007.

5. Jansen JP, Crawford B, Bergman G, et al. Bayesian meta-analysis of multiple treatment comparisons: an introduction to mixed treatment comparisons. Value Health 2008;11:956-64.

6 Mortimer D, Segal L. Comparing the incomparable? A systematic review of competing techniques for converting descriptive measures of health status into QALY-weights. Med Decis Making 2007;28(1):66-89.

7. Karvetski CW, Lambert JH, Linkov I. Scenario and multiple criteria decision analysis for energy and environmental security of military and industrial installations. Integr Environ Assess Manage 2011;7(2):228-36.

8. Mavrotas G, Ziomas IC, Diakouaki D. A combined MOIP-MCDA approach to building and screening atmospheric pollution control strategies in urban regions. Environ Manage 2006 Jul;38(1):149-60.

9. Thokala P, Duenas A. Multiple criteria decision analysis for health technology assessment. Value Health 2012;15(8):1172-81.

10. Littlejohns P, Sharma T, Jeong K. Social values and health priority setting in England: "values" based decision making. J Health Organ Manage 2012;26(3):363-73.

11. Postmus D, Tervonen T, van Valkenhoef G, Hillege HL Buskens E. A multi-criteria decision analysis perspective on the health economic evaluation of medical interventions. Eur J Health Econ 2014;15:709-16.

12. Figueira J, Greco S, Ehrgott M, editors. Multiple Criteria Decision Analysis: State of the Art Surveys Series. New York: Springer; 2005.

13. Baltussen R, Niessen L. Priority setting of health interventions: the need for multi-criteria decision analysis. Cost Effect Res Alloc 2006;4:14.

14. Wade SW, Strader C, Fitzpatrick LA, Anthony MS, O'Malley CD. Estimating prevalence of osteoporosis: examples from industrialized countries. Arch Osteoporos 2014;9:182.

15. Piscitelli P, Brandi M, Cawston H, Gauthier A, Kanis JA, Compston J, et al. Epidemiological burden of postmenopausal osteoporosis in Italy from 2010 to 2020: estimations from a disease model. Calcif Tissue Int 2014 Nov;95(5):419-27.

16. Hernlund E, Svedbom A, Ivergård M, Compston J, Cooper C, Stenmark J, et al. Osteoporosis in the European Union: medical management, epidemiology and economic burden. A report prepared in collaboration with the International Osteoporosis Foundation (IOF) and the European Federation of Pharmaceutical Industry Associations (EFPIA). Arch Osteoporos 2013;8(1):136.

17. THE EUROPEAN PROSPECTIVE OSTEOPOROSIS STUDY (EPOS) GROUP. Incidence of vertebral fracture in Europe: results from the European Prospective Osteoporosis Study. J Bone Miner Res 2002;17:716-24.

18. Migliore A, Integlia D, Bizzi ., Piaggio T. Is it the time to rethink clinical decision-making strategies? Froma a single clinical outcome evaluation to a Clinica Multicriteria Decision Assessment (CMDa), Med Hypotheses (2015).

19. Kling JM, Clarke BL, Sandhu NP. Osteoporosis prevention, screening, and treatment a review. J Womens Health (Larchmt) 2014;23(7):563-72.

20. Lyles KW, Colón-Emeric CS, Magaziner JS, Adachi JD, Pieper CF, Mautalen C, et al. HORIZON Recurrent Fracture Trial. Zoledronic acid and clinical fractures and mortality after hip fracture. N Engl J Med 2007;357(18): 1799-809.

21. Shiligsmann M, Rabenda V, Gathon HJ, Ethgen O, Reginster JY. Potential clinical and economic impact of nonadherence with osteoporosis medications. Calcif Tissue Int 2010;86(3):202-10.

22. Brooks PM. The burden of musculoskeletal disease-a global perspective. Clin Rheumatol 2006 Nov;25(6): 778-81.

23. Helmick CG, Felson DT, Lawrence RC, et al. Estimates 
of the prevalence of arthritis and other rheumatic conditions in the United States. Part I. Arthritis Rheum 2008;58:15-25.

24. Gaujoux-Viala Cécile, Smolen Josef S, Landewé Robert, Dougados Maxime, Kvien Tore K, Mola Emilio Martin, et al. Current evidence for the management of rheumatoid arthritis with synthetic disease-modifying antirheumatic drugs a systematic literature review informing the EULAR recommendations for the management of rheumatoid arthritis. Ann Rheum Dis 2010. 


\title{
Il metodo clinico in condizioni di incertezza: errori, vincoli, esperienza, buon senso e le opinioni del paziente
}

\author{
Maria Luigia Cipollini, Elisabetta Romboli \\ Medicina A Cardio-Renale, Ospedale Maggiore - Ausl Bologna, Italia
}

Ippocrate: con innocenza e purezza custodirò la mia vita e la mia arte.

Ippocrate è il padre fondatore dell'ars medica antiqua e, in specifico, l'arte di ristabilire l'equilibrio, l'armonia e la salute. Ma già dai tempi degli antichi greci, scienza ed arte medica non potevano essere separate, coniugandosi in un equilibrato intersecarsi tra ragionamento ed esperienza. Nei tempi più moderni la Medicina si è invece identificata sempre più come scienza e il metodo clinico è diventato l'applicazione spesso acritica delle evidenze scientifiche. Attualmente però il Clinico ha dovuto rimettere in discussione il suo modo di essere Medico e riscoprire la necessità di calare il suo sapere scientifico sul paziente, che è prima di tutto persona con specifiche necessità, bisogni, timori con esigenze di ascolto e comprensione. Siamo di fronte ad una nuova fase in cui necessita un cambiamento della mentalità generale con una riscoperta del valore di creare un rapporto di empatia col paziente, che permetta di creare quella relazione di fiducia imprescindibile per una buona riuscita di un processo terapeutico.

Il medico peraltro sempre più si trova ad operare in condizioni di incertezza soprattutto in specifici contesti come la Medicina Interna, dove la complessità rappresenta la regola e non l'eccezione. Le condizioni di certezza sono infatti rappresentate da quelle situazioni in cui vi è una unica patologia e per le quale vi sono ampie dimostrazioni consolidate di provata efficacia di una specifica terapia. Le

Corrispondente: Elisabetta Romboli, UOS Cardio-Renale, Medicina Interna A - Dipartimento Medico, Ospedale Maggiore, Azienda USL Bologna, Largo Bartolo Nigrisoli 2, 40133 Bologna, Italia.

Tel. +39 051-6478074. Fax +39 051-2172536.

E-mail: e.romboli@ausl.bo.it

Articolo pubblicato secondo la Creative Commons Attribution NonCommercial 4.0 License (CC BY-NC 4.0).

${ }^{\circ}$ Copyright: the Author(s), 2020

Licensee PAGEPress, Italy

QUADERNI - Italian Journal of Medicine 2020; 8(1):67-72 condizioni di incertezza non riguardano soltanto quelle situazioni in cui non sono disponibili provate documentazioni: in questi contesti il medico deve orientarsi su criteri probabilistici, e pertanto non assoluti, ma soprattutto deve orientare le proprie decisioni in base a diverse variabili non solo biologiche ma anche psico-sociali ed economiche. Il giudizio clinico deve coniugare il pensiero, partendo da basi fisiopatologiche e conoscenze scientifiche, ma integrando queste con alcune priorità quali le opinioni del paziente nell'ambito del contesto sociale, normativo ed etico. Sono proprio queste situazioni quelle di gran lunga più frequenti in Medicina Interna e che condizionano ed orientano le decisioni del medico internista. ${ }^{1} \mathrm{Si}$ opera infatti con soggetti anziani spesso pluripatologici, plurifarmaco-trattati, non di rado con disabilità motorie e cognitive $\mathrm{e}$ in contesti sociali non ottimali ed il clinico deve gestire questa complessità ovvero quando le differenti componenti che costituiscono un tutto sono inseparabili e quando vi è un tessuto interdipendente e interattivo fra le parti e il tutto, e il tutto e le parti. ${ }^{2}$ La complessità richiede una strategia, che è l'arte di muoversi nell'incertezza. Complessità significa impossibilità da parte della scienza di ridurre in termini lineari la realtà oggetto di studio, ovvero aspira alla conoscenza multidimensionale e multidisciplinare, ma è consapevole dell'impossibilità della conoscenza completa. Come ricorda Wilson il pensiero complesso richiede un equo giudizio circa le possibili interazioni tra i diversi componenti in causa. ${ }^{3}$ La complessità rende la Medicina una scienza probabilistica, con un elevato rischio di errore, per l'incertezza che permea le decisione mediche, che devono essere adottate in un tempo limitato ed in un contesto di conoscenza non sempre definito. La definizione di paziente complesso, adottata dall'Agency for Healthcare Research and Quality (AHRQ), si riferisce a una persona affetta da due o più malattie croniche, in cui ciascuna delle condizioni morbose presenti è in grado d'influenzare l'esito delle cure delle altre coesistenti, attraverso varie modalità: la limitazione della speranza di vita, l'aumentata morbilità intercorrente, le interazioni tra le terapie farmacologiche, l'impossibilità del pieno impiego di cure adeguate per controindicazione. ${ }^{4}$ 
Nell'accezione più completa di complessità, le componenti di tipo biologico, socio-economico, culturale, comportamentale ed ambientale diventano tutte importanti determinanti di salute, purtroppo e paradossalmente considerate, nei criteri di eleggibilità, come potenziali confondenti ai fini di un'oggettiva valutazione dei risultati di un trial clinico. La complessità pone all'internista non solo un miglioramento nelle sue competenze (Tabella 1), ma anche della sua adattabilità al cambiamento, attraverso nuove conoscenze e con i massimi livelli di consapevolezza. La valutazione clinica deve prendere in considerazione tutte quelle variabili personali di contesto e di bisogni del singolo paziente, finalizzato ad una decisione più adeguata in considerazione dell'unicità del processo assistenziale. ${ }^{5}$

Nell'ultima decade del secolo scorso abbiamo assistito ad un cambiamento epocale ovvero al passaggio, da un metodo clinico empirico, alla medicina basata sulle evidenze (EBM), determinando, in diverse generazioni di clinici, un cambiamento culturale radicale in tutti gli ambiti di assistenza sanitaria, pratica professionale, formazione, pianificazione della politica sanitaria e della ricerca, comunicazione con pazienti e cittadini. ${ }^{6} \mathrm{Ma}$ l'EBM presenta alcuni limiti di applicabilità nel mondo reale. Le basi su cui si poggia la medicina basata sulle evidenze sono i risultati dei trials clinici controllati (RCT) ovvero di studi eseguiti su popolazione selezionata. L'applicabilità di uno studio clinico randomizzato (RCT), ovvero la sua validità esterna è il grado con cui i risultati della ricerca possono essere applicati al paziente individuale. Essa è influenzata principalmente dai criteri di selezione/esclusione dei pazienti dello studio, dal setting assistenziale, dagli aspetti organizzativi, dalle tecnologie, dalla clinical competence, dalla capacità del paziente di aderire alle cure, ma anche dalla significatività e rilevanza clinica dei risultati ottenuti. Uno dei problemi più critici, inerente la generalizzazione dei risultati di un RCT, riguarda i criteri di eleggibilità/esclusione da uno studio. Il profilo del paziente tipo ammissibile per uno studio clinico corrisponde, in generale, a un soggetto di sesso maschile, giovane adulto, affetto da una sola malattia, acuta o subacuta, senza compromissioni funzionali, con un'ottima compliance, con follow up di durata limitata, con valutazione dell'efficacia piuttosto che della sicurezza/tollerabilità, controllato con placebo, con endopoint surrogati. ${ }^{7}$

Al contrario, il mondo reale dei pazienti ricoverati in ospedale è più spesso costituito da persone di sesso femminile, affette da malattie croniche degenerative con associate frequenti sindromi geriatriche (cadute, delirium, demenza, incontinenza urinaria, dolore cronico, depressione) e numerose altre comorbilità, perdita dell'autonomia ed in trattamento polifarmacologico. Proprio per questo motivo l'EBM ha una diffusione disomogenea nella pratica medica. Secondo dati più recenti, con riferimento ai medici di varie specializzazioni, la percentuale delle decisioni basate sulle regole dell'EBM varia dall' 11 al $70 \%{ }^{8}$ Gli specialisti d'organo e/o di apparato, tendono ad

Tabella 1. La gestione del paziente complesso in Medicina Interna.

\begin{tabular}{|c|c|}
\hline Caratteristiche del paziente internistico & Competenze richieste all'internista \\
\hline - Adulti, generalmente anziani o molto anziani & - Valutazione clinica, laboratoristica, strumentale e multidimensionale \\
\hline - Con diverse co-morbidità & $\begin{array}{l}\text { - Inquadramento nel setting assistenziale (attuale e futuro) e valutazione } \\
\text { della situazione }\end{array}$ \\
\hline - Con prevalenti malattie croniche/cronico-riacutizzate & - Stratificazione del rischio e selezione degli elementi salienti \\
\hline - In alcuni casi robusti & - Definizione delle priorità e dei bisogni \\
\hline - In alcuni casi fragili & - Selezione degli obiettivi di cura e della terapia farmacologica appropriata \\
\hline - A volte disabili, a rischio di perdita dell'autosufficienza & - Valutazione evidence based delle prove di efficacia disponibili \\
\hline - A volte instabili, in alcuni casi critici & - Decision making in condizioni d'incertezza \\
\hline - Con limitata speranza di vita & - Stratificazione prognostica \\
\hline - In trattamento poli-farmacologico & - Relazioni con i famigliari e i caregiver \\
\hline - Con frequenti ricoveri & - Relazioni e interazioni con gli specialisti \\
\hline - In alcuni casi a rischio di dimissione difficile & - Educazione al self-management \\
\hline - Che richiedono il giusto tempo di ascolto e di valutazione & - Coordinamento e comunicazione \\
\hline - Che richiedono l'intervento di diversi specialisti & - Pianificazione del case management \\
\hline - Condivisi fra diversi medici nel processo di assistenza & - Continuità assistenziale \\
\hline - Che richiedono continuità assistenziale & \\
\hline
\end{tabular}


utilizzare maggiormente l'EBM, probabilmente per il più limitato campo d'azione, in riferimento a pazienti con problemi omogenei, ben rappresentati nei grandi trial clinici, al contrario di quelli più complessi trattati dagli internisti. ${ }^{9}$ A queste limitazioni deve aggiungersi quanto descritto da Chang e Lee, ${ }^{10}$ ovvero l'imprescindibile integrazione delle linee guida basate su dati biologici derivanti dalla ricerca clinica con le norme operative, i programmi assicurativi e di incentivazione $e$, non ultimo, i sistemi di informazione. Ed è proprio per questo motivo che non possono essere applicate ad ogni caso come un abito che vada bene per ogni taglia.

Nel 1996 Sackett definì l'EBM come l'uso coscienzioso, esplicito e giudizioso delle attuali migliori prove nel prendere decisioni sulla cura dei singoli pazienti; in tal senso le linee guida sono da considerare degli indirizzi a cui riferirsi e non l'applicazione acritica di una regola. L'applicazione corretta delle linee guida non consiste nel trasferire in maniera automatica le acquisizioni sperimentali alla pratica clinica, ma sapere integrare l'esperienza clinica individuale con le migliori conoscenze derivanti dalla revisione sistematica degli studi clinici relativi a un determinato argomento. Il metodo clinico deve pertanto essere una sintesi tra la conoscenza delle migliori evidenze presenti in letteratura e l'esperienza professionale del medico. Il Clinico deve avere la capacità di valutare la trasferibilità e l'appropriatezza dei dati sperimentali al singolo paziente, tenendo conto dei suoi valori, delle sue preferenze, del contesto socio-assistenziale e, non ultimo, della sostenibilità economica delle sue scelte diagnostico-terapeutiche.

Il Clinico attualmente, e in particolare l'Internista, deve raffrontarsi con una popolazione di pazienti che spesso discosta dalle caratteristiche dei pazienti scelti nei trials, in genere eseguiti su popolazioni altamente selezionate. È dimostrato infatti per esempio che gli anziani in genere non vengono arruolati nei trials clinici pubblicati su riviste ad alto impact factor in Medicina Interna, dove l'età rappresenta la principale motivazione per l'esclusione dagli RCT nel 72\% dei casi. ${ }^{11}$ Inoltre, non sempre sono disponibili evidenze in grado di dimostrare l'efficacia di una determinata terapia per quella specifica malattia. Ad esempio, in ambito terapeutico, considerando l'efficacia e/o potenziale nocività di diversi tipi di trattamento è stato documentato che solo il $38 \%$ degli interventi può essere ritenuto utile o potenzialmente utile. Prove chiare, sia di segno positivo (utili), che negativo (verosimilmente inefficaci e dannose) possono essere fornite solo nel 19\% dei casi. ${ }^{12}$ Questi dati confermano le condizioni di incertezza del mondo reale in cui l'internista è chiamato ad operare. In questi casi è necessario basarsi su prove meno dirette, come: i) l'osservazione clinica di gruppi di pazienti che hanno avuto risultati differenti con cure diverse somministrate nella pratica quotidiana e non in uno studio mirato; ii) studi sulle popolazioni che hanno dimostrato come determinati comportamenti sono efficaci; iii) riunioni di consenso fra esperti che, sulla base di dati scientifici indicativi ma non conclusivi e certi, indicano comunque il più ragionevole atteggiamento da tenere nella pratica clinica quotidiana.

Data la complessità del mondo reale in cui il Clinico si trova ad agire, in particolare il Medico Internista, i risultati dei grandi trials per quanto rigorosi, risulta difficile applicare i loro risultati in modo meccanico ed indiscriminato. Certamente offrono un'indicazione su quelle che sono con alta probabilità statistica le migliori terapie per tutti, ma il clinico dovrà valutare quali possono essere i rischi ed i benefici di quello specifico intervento in quel particolare paziente. Andranno considerate le caratteristiche specifiche del paziente considerando non solo quella specifica malattia ma il corollario di patologie di cui è affetto il paziente e i numero farmaci che quel paziente deve assumere, valutando interazioni ed effetti collaterali possibili. Inoltre, andranno considerate le sue esigenze, le sue preoccupazioni, aspettative e preferenze. L'Art. 1 della legge 219-17 promuove e valorizza la relazione di cura e di fiducia tra medico e paziente che si basa sul consenso informato nel quale si incontrano l'autonomia decisionale del paziente e la competenza, l'autonomia professionale e la responsabilità del medico. Il processo decisionale di cura va condivisa con il paziente, non può prescindere dal dialogo o meglio dalla costituzione di una alleanza medicopaziente, conditio sine qua non per una congrua aderenza all'iter diagnostico-terapeutico proposto e concordato. Senza l'alleanza terapeutica medicopaziente si perde l'efficacia anche del farmaco migliore, che può non essere assunto, essere assunto irregolarmente, $\mathrm{o}$ addirittura non essere accettato. Non a caso anche le prescrizioni di farmaci risultati efficaci nei trials clinici controllati ad un anno di distanza la compliance dei pazienti non supera il 50\%. In sintesi, non deve essere spostato l'asse medico-paziente verso la direttrice medico-ricerca clinica. Il medico che applica i principi della Medicina Basata sulle Evidenze deve cercare le migliori soluzioni possibili, servendosi delle prove scientifiche di efficacia di maggior valore presenti nella letteratura medica. Le deve però applicare criticamente, alla luce della sua esperienza e abilità ed in funzione dell'assistenza mirata a quel singolo paziente.

In una medicina incentrata sui pazienti del mondo reale, la migliore evidenza non può peraltro derivare solamente dagli RCT ma anche da studi osservazionali ben condotti che i medici dovranno saper interpretare 
nella loro pratica clinica. ${ }^{13}$ Come ricorda Van Spall, ${ }^{11}$ per decisioni cliniche adeguate è necessario poter disporre, oltre che di trials finalizzati a dimostrare l'efficacia degli interventi, anche di studi pragmatici in grado di documentarne l'applicabilità. L'obiettivo della medicina centrata sul paziente è quello di fare una prescrizione patient tailored ma questo contrasta con i principi dell'EBM per l'impossibilità di confrontare $\mathrm{i}$ benefici di terapie in presenza di trattamenti concomitanti e prognosi differenti. Le future revisioni sistematiche dovranno integrare i dati ottenuti dagli RCT e dagli studi osservazionali al fine di valutare efficacemente sia i benefici sia i danni delle terapie. ${ }^{14}$

In merito agli studi e ai dati che emergono, è necessario valutare anche il peso e il significato di concetti che possono avere un valore diverso se analizzato dal ricercatore-epidemiologo o dal clinico nella pratica quotidiana e ne è un esempio il concetto di significatività statistica. Significatività statistica non sempre esprime un risultato con rilevanza clinica: risultati statisticamente significativi possono essere clinicamente non importanti e, viceversa, effetti terapeutici statisticamente non significativi possono veicolare il messaggio di una terapia clinicamente utile. La significatività statistica è un concetto probabilistico ovvero rappresenta la probabilità di ottenere un falso positivo quando si afferma che esiste un certo effetto. Per convenzione, per sostenere che un effetto è statisticamente significativo, questa probabilità deve essere inferiore al $5 \%(\mathrm{P}<0.05)$. La rilevanza statistica degli studi è riportata in termini di outcomes pesanti (mortalità totale o causa-specifica, eventi non fatali, morbilità, number needed to treat, ecc.). Il clinico peraltro deve valutare se, oltre a questi outcomes, vi possono essere altri indicatori altrettanto importanti e soprattutto utili per quello specifico paziente tali da indurre cambiamenti in alcune prescrizioni anche se il confronto non ha mostrato significatività per quanto riguarda gli outcomes primari (mortalità, morbilità, etc.).

I trials clinici controllati (RCT) rappresentano le basi, quando disponibili, delle Linee Guida di numerose patologie di ampio impatto nella popolazione. Ma qual è la qualità di queste Linee Guida? Sono concordanti le Linee Guida riguardanti specifiche patologie da parte di diverse Società Scientifiche? Quante di queste Linee Guida non sono basate su studi clinici controllati ma su opinione di esperti? Questi quesiti sono estremamente attuali anche in riferimento a recenti ordinamenti legislativi (Legge Gelli sulla Responsabilità Professionale del Medico - Legge $n^{\circ} 24 ; \mathrm{GU}^{\circ} 64 \mathrm{del}$ 17.03.2017) nei quali avere seguito o non seguito le raccomandazioni delle Linee Guida rappresenta il discrimine per giudicare la colpa/non colpa del medico. Le Linee Guida possono presentare diverse limitazioni
(Tabella 2) in quanto sono troppo spesso incentrate sulle singole patologie e non sono mirate al singolo paziente. Le raccomandazioni basate sulle evidenze sono in molti casi non definitive e a volte addirittura contrastanti, mentre le decisioni del medico devono essere univoche e rapide. Si può pertanto affermare che l'Evidence Based Medicine è necessaria ma non sufficiente per la pratica clinica. ${ }^{15}$ Sono indispensabili ulteriori nuove evidenze, impostate sia su studi clinici controllati e/o osservazionali maggiormente applicabili alla realtà di tutti i giorni. I pazienti raramente hanno singole malattie e sono più uniche che rare le Linee Guida che sono in grado di supportare il medico nel gestire in maniera flessibile i casi più complessi e garantire la personalizzazione delle cure. Infatti, essendo strutturate per trattare pazienti con una singola malattia, ${ }^{16}$ possono paradossalmente rendere problematica la decisione del clinico nei pazienti pluripatologici. Anzi, è stato documentato che una pedissequa adesione alle linee guida negli anziani può avere effetti secondari rilevanti. ${ }^{17,18}$ Attenzione però a limitare l'importanza della EBM o, ancor peggio, a darne una connotazione negativa tale da indurre allo scetticismo o addirittura all'inerzia decisionale. Nel mondo della complessità, intesa come ambito d'azione avere questa consapevolezza rappresenta una barriera razionale contro il rischio di errore. Le linee guida basate su RCT rappresentano la base delle conoscenze mediche ma anche con queste premesse il medico internista non deve perdere la capacità critica che ha sempre contraddistinto il metodo clinico che ha avuto come antesignano Augusto Murri: egli deve estrapolare gli elementi più solidi dalle LG, per evitare errori. ${ }^{19}$ In sostanza, il medico, e nello specifico l'internista, deve essere in grado di estrapolare dalla complessità del paziente il problema prevalente e individuare la best available evidence. Nella valutazione dell'efficacia clinica delle scelte d'intervento, l'internista dovrebbe sempre chiedersi se queste funzionano e producono più effetti positivi che negativi, considerandone gli effetti in relazione alle risorse utilizzate ed ai rischi potenziali e/o attuali. Il valore aggiunto all'EBM è rappresentato proprio dalla integrazione dei dati evidence-based con l'esperienza, il buon senso e soprattutto con la condivisione delle decisioni cliniche con il paziente tenendo conto dei suoi valori e delle sue preferenze (Tabella 3). Coinvolgere il paziente nella cura è un processo importante, per esempio nella definizione dei patient-reported outcomes ma nel complesso non sempre applicabile in quanto diversi elementi interferiscono quali emozioni, disfunzione cognitiva, depressione, stato di coscienza, presenza di più patologie organiche, stato sociale ed economico, solitudine, rendendo difficile il normale coinvolgimento del paziente. ${ }^{20}$ Non tutti desiderano infatti essere coinvolti alla stessa maniera nel processo di cura, a 
volte anche per una mancato adattamento psicodinamico alla malattia ed un rifiuto alla stessa. Le persone hanno un vissuto, con priorità e valori che, nella vita reale, incidono sulle decisioni diagnostiche e terapeutiche, nonché sulla prognosi. Per il medico è difficile non solo valutare efficacemente i desideri del paziente, ma anche trasmettere informazioni necessarie che sono quasi sempre fondate su criteri probabilistici non facilmente comprensibili da parte dei pazienti. D'altra parte la letteratura medica è povera di riferimenti su come $\mathrm{i}$ medici possano riuscire a condividere le evidenze della letteratura scientifica con $\mathrm{i}$ loro pazienti per le decisioni del caso. Ed è proprio in questo sentiero impervio che il clinico deve addentrarsi per allargare le proprie competenze e sviluppare la comprensione del malato, della famiglia e delle sue aspettative. La spiegazione semplice delle conoscenze disponibili integrata dalla formulazione esplicita del proprio parere, il controllo della comprensione di quanto discusso per una buona riuscita del piano diagnostico-terapeutico rappresentano le basi per costruire una solida alleanza fra curante e paziente. ${ }^{21}$ Questa alleanza genera nel paziente la consapevolezza di essere curato secondo una metodologia rigorosa volta a dargli sempre il meglio, scegliendo esami diagnostici e terapia di dimostrata efficacia: ovvero una medicina che viene definita interpersonal medicine incentrata sul paziente di cui il medico deve occuparsi come persona e non come malattia. ${ }^{10}$ Spesso la soluzione di un disturbo apparentemente organico, richiede al medico di affrontare fattori comportamentali e sociali attraverso una serie di interazioni quali l'impegno, la convinzione e la resilienza che si verificano a casa, al lavoro, negli ospedali tra clinici e pazienti e fra pazienti e famiglie. Attraverso tali interazioni, vengono costruite delle relazioni che permettono al paziente di superare

Tabella 2. Principali limitazioni delle attuali linee guida.

\begin{tabular}{l}
\hline Sono orientate a una specifica patologia \\
\hline - Sono basate su RCT selezionati, raramente dedicati al paziente anziano con co-morbidità \\
\hline - Non sono basate su studi condotti in pazienti complessi e fragili, le raccomandazioni sono limitate al paziente ideale \\
\hline$-\quad$ Le raccomandazioni non contemplano l'eterogeneità del paziente in termini di: \\
1) Comorbilità \\
2) Poli-farmacoterapia \\
3) Stato funzionale e cognitivo \\
4) Stato socio-familiare \\
5) Setting assistenziale
\end{tabular}

Tabella 3. Differenze tra Medicina Basata sulle Evidenze e ma Medicina Incentrata sul paziente.

\begin{tabular}{|c|c|}
\hline Medicina Basata sulle Evidenze & Medicina Incentrata sul paziente \\
\hline Disease-centered & Patient centered \\
\hline $\begin{array}{l}\text { - Viene valutata la migliore evidenza disponibile in riferimento } \\
\text { alla malattia }\end{array}$ & $\begin{array}{l}\text { - Viene valutata in base a bisogni, alle aspettative del paziente e alla } \\
\text { valutazione del rapporto beneficio/rischio }\end{array}$ \\
\hline $\begin{array}{l}\text { - Approccio biomedico: integrazione delle evidenze scientifiche } \\
\text { con l'esperienza del medico }\end{array}$ & $\begin{array}{l}\text { - Approccio olistico umanistico, interattivo e empatico con il } \\
\text { paziente, con applicazione delle evidenze scientifiche (quando e se } \\
\text { disponibili) in base a una giusta valutazione dei problemi prioritari } \\
\text { e con le aspettative del paziente }\end{array}$ \\
\hline $\begin{array}{l}\text { - Base sperimentale forte, prevalentemente mediante studi clinici } \\
\text { randomizzati effettuati in gruppi di pazienti omogenei }\end{array}$ & $\begin{array}{l}\text { - Base pragmatica forte, prevalentemente fondata su studi } \\
\text { osservazionali in pazienti appartenenti al mondo reale }\end{array}$ \\
\hline $\begin{array}{l}\text { - Criteri di ammissione/esclusione rigidi dei pazienti negli studi } \\
\text { clinici con selezione metodologicamente corretta dei pazienti } \\
\text { e valutazione della significatività statistica }\end{array}$ & $\begin{array}{l}\text { - La comorbilità e complessità dei pazienti del mondo reale } \\
\text { richiedono esperienza, capacità discriminativa degli elementi clinici } \\
\text { salienti, ovvero rilevanza clinica piu`che statistica }\end{array}$ \\
\hline - $\quad$ Il paziente non interagisce mai con lo sperimentatore & $\begin{array}{l}\text { - Il paziente è a fianco del clinico, che deve decidere in un prevalente } \\
\text { contesto di complessità, in una relazione di cura orientata all'ascolto } \\
\text { dei bisogni del paziente }\end{array}$ \\
\hline $\begin{array}{l}\text { - Prevale il giudizio sul metodo: si valuta se vengono rispettati i } \\
\text { consolidati obiettivi della ricerca metodologicamente corretta }\end{array}$ & $\begin{array}{l}\text { - Prevale il giudizio clinico: ovvero saper selezionare le priorità per } \\
\text { quel paziente, in base a un ragionamento sia fisiopatologico, sia di } \\
\text { valutazione multidimensionale, contestualizzato al setting } \\
\text { assistenziale }\end{array}$ \\
\hline $\begin{array}{l}\text { - In sintesi: prevale l'attenzione alla validità metodologica } \\
\text { dello studio }\end{array}$ & $\begin{array}{l}\text { - In sintesi: si richiede di valutare al letto del malato nel paziente } \\
\text { reale l'applicabilità dei risultati degli studi clinici }\end{array}$ \\
\hline
\end{tabular}


determinati ostacoli. La medicina interpersonale richiede abilità da parte del clinico nel sedersi accanto al paziente lungo il percorso della malattia, come compagno di viaggio ma anche guida esperta. Vari studi clinici hanno dimostrato come siano fondamentali l'empatia, la coordinazione e la motivazione; non sorprende infatti che una migliore comunicazione da parte del medico è associata ad un aumento del 19\% nell'aderenza dei pazienti alla terapia. Possiamo pertanto perseguire una versione empatica della medicina che abbraccia l'emozione e apprezza il comportamento, se valutiamo la natura umana tanto quanto la biologia umana. E allora è necessario che riscopriamo la medicina come arte, che avvenga quel salto di mentalità con un recupero di quella sensibilità che deve contraddistinguere l'operato medico con la consapevolezza e la coscienza che il paziente nella sua complessità rappresenta un'unicità da scoprire, comprendere e curare alla luce però delle evidenze scientifiche che la letteratura propone che vanno calate e interpretate in quello specifico e peculiare contesto.

\section{Bibliografia}

1. Nardi R, Fabbri T, Belmonte G et al. Medicina Interna, paziente complesso, evidence based e le non evidenze, It J Med 2009; 3: 191-200.

2. Morin E. I sette saperi necessari all'educazione del futuro. Milano: Raffaello Cortina; 2001.

3. Wilson T, Holt T, Greenhalgh T. Complexity science: complexity and clinical care. BMJ 2001; 323 (7314): 685-8; Plsek PE, Wilson T. Complexity, leadership, and management in healthcare organisations. BMJ 2001; 323 (7315): 746-9.

4. Agency for Healthcare Research and Quality (AHRQ). Definition: Complex Patient, Funding Opportunity Announcement (FOA). Technical Assistance Conference Call. October 15, 2007.

5. Fraser SW, Greenhalgh T. Coping with complexity: educating for capability. BMJ 2001; 323 (7316): 799-803.

6. Cartabellotta A. La medicina basata sulle evidenze: criticità e prospettive. Recenti Prog Med 2006; 97 (11): 640-6.
7. Gambassi G. EBM o Evidence Biased Medicine. Dai trial clinici al mondo reale. 538 Congresso Nazionale SIGG, XIII Congresso Nazionale FADOI, 2008.

8. Pelletier K. Conventional and integrative medicine: evidence based? Sorting fact from fiction. Focus Alternative Complement Ther 2003;8 (1): 3-6.

9. Gill P, Dowell AC, Neal RD, Smith N, Heywood P, Wilson AE. Evidence based general practice: a retrospective study of interventions in one training practice. BMJ 1996;312(7034): 819-21.

10. Chang S, Lee TH. Beyond Evidence-Based medicine, N Eng J Med 379;21:2018.

11. Van Spall HG, Toren A, Kiss A, Fowler RA. Eligibility criteria of randomized controlled trials published in high-impact general medical journals: a systematic sampling review. JAMA 2007; 297 (11):1233-40.

12. Liberati A. Centro Cochrane Italiano, XIII Riunione Annuale Network Cochrane Italiano. Workshop. Napoli, 4 novembre 2008.

13. D’Agostino Jr RB, D'Agostino Sr RB. Estimating treatment effects using observational data. JAMA 2007; 297 (3): 314-6.

14. Vandenbroucke JP, Psaty BM. Benefits and risks of drug treat- ments: how to combine the best evidence on benefits with the best data about adverse effects. JAMA 2008;300(20): 2417-9.

15. Tonelli MR. The philosophical limits of evidence-based medi- cine. Acad Med 1998;73 (12): 1234-40.

16. Shaneyfelt TM, Centor RM. Reassessment of clinical practice guidelines: go gently into that good night. JAMA 2009;301 (8): 868-9.

17. Van Weel C, Schellevis FG. Comorbidity and guidelines: conflicting interests. Lancet 2006;367 (9510): 550-1.

18. Tinetti ME, Bogardus Jr ST, Agostini JV. Potential pitfalls of disease-specific guidelines for patients with multiple conditions. N Engl J Med 2004;351(27):2870-4.

19. O'Connor PJ. Adding value to evidence-based clinical guide-lines. JAMA 2005;294 (6): 741-3.

20. Heath I, Rubinstein A, Stange KC, van Driel ML. Quality in primary health care: a multidimensional approach to complexity. BMJ 2009; 338: b1242.

21. Epstein RM, Alper BS, Quill TE. Communicating evidence for participatory decision making. JAMA 2004;291 (19): 2359-66. 


\section{Meta-cognizione e capacità di decision making}

Fabio Gilioli

UOC Medicina Interna, Ospedale S. Maria S. Maria Bianca, AUSL di Modena, Mirandola (MO), Italia

\section{Introduzione}

Negli Stati Uniti si stima che ogni anno vi siano da 44.000 a 98.000 pazienti deceduti per errori medici, spesso di natura diagnostica, responsabili fino al 17\% di tutti gli eventi avversi. ${ }^{1-3}$ La diagnosi è l'esito di un processo decisionale (decision making) determinato dal ragionamento clinico del medico. La logica conseguenza é che la maggior parte degli errori sia dovuta agli aspetti del ragionamento clinico correlabili, in base alla recente letteratura. a pregiudizi cognitivi (cognitive biases) definibili come deviazioni dal giudizio razionale dell'individuo. ${ }^{4,5}$ Sono stati proposti molti metodi per ridurre la presenza di cognitive biases. La metacognizione è una di queste strategie e viene ampiamente utilizzata sia in ambito educativo che psicologico. Essa può essere espressa come la consapevolezza ed il controllo che un soggetto ha dei processi cognitivi, un'abilità descritta dagli autori anglosassoni come thinking about thinking ${ }^{4-6}$ ma che raramente viene insegnata nel corso degli studi universitari di medicina. Il tema é sicuramente complesso quindi lo scopo di questo articolo è di fornire solo alcuni elementi di riflessione sul ruolo della metacognizione sia nei processi di decision making che nell'attività formativa dei futuri medici.

\section{Pensiero intuitivo e pensiero meditato}

Da alcuni anni, in particolare dopo gli studi di Gladwel $^{7}$ e Kahneman ${ }^{8}$ prevale la teoria che l'attività

Corrispondente: Fabio Gilioli, UOC Medicina Interna, Ospedale S. Maria S. Maria Bianca, via Antonio Fogazzaro 6, 41037 Mirandola (MO), Italia.

E-mail: f.gilioli@ausl.mo.it

Articolo pubblicato secondo la Creative Commons Attribution NonCommercial 4.0 License (CC BY-NC 4.0).

${ }^{\circ}$ Copyright: the Author(s), 2020

Licensee PAGEPress, Italy

QUADERNI - Italian Journal of Medicine 2020; 8(1):73-80 mentale dell'uomo si esplichi attraverso due modalità di pensiero definite dagli psicologi Keith Stanovich e Richard West ${ }^{9}$ sistema 1 e il sistema 2 rispettivamente uno di carattere intuitivo ed uno meditato.

Il sistema 1 è utilizzato frequentemente con poco sforzo e nessun controllo volontario. Ha competenze innate quali la percezione del mondo intorno a noi, riconosce oggetti e orienta l'attenzione. Il nucleo centrale della sua attività è basato sulla memoria associativa che consente di distinguere in una frazione di secondo gli eventi normali da quelli sorprendenti e cerca una interpretazione causale degli eventi che si verificano. Le attività mentali, con la pratica diventano veloci e automatiche, consentono di sviluppare competenza, capacità di giudizio e quindi permettono di effettuare una scelta conclusiva con modalità intuitiva, spesso anche accurata. Le peculiarità del sistema 1 presentano limiti caratterizzati da una ridotta capacità di applicare ragionamenti statistici (probabilità e casualità sono concetti sostituiti da quelli più semplici basati sulla causalità), scarsa propensione all'incertezza e dubbio, tendenza a preferire riflessioni basate su esperienze ripetute, emotivamente piacevoli, processi cognitivi fluidi e ha quindi maggiore facilità a saltare a conclusioni premature preoccupandosi meno di ricercare informazioni o conferme. $^{8} \mathrm{Di}$ fatto vengono utilizzate scorciatoie mentali tipiche del pensiero euristico. (il termine da cui trae origine ha la stessa radice di eureka ed è il verbo greco heurìskein, trovare). Kahneman ${ }^{8}$ definisce l'Euristica, su cui torneremo successivamente, una semplice procedura che aiuta a trovare risposte adeguate, anche se imperfette, a questioni difficili.

Il sistema 2 invece è lento, logico, rivolto ad attività mentali impegnative come le elaborazioni di calcoli, richiede concentrazione e un pensiero cosciente. Le operazioni automatiche del sistema 1 generano modelli di idee molto complessi, ma solo il sistema 2 è in grado di elaborare pensieri in una serie ordinata di stadi.

Nella maggior parte del tempo, il sistema 2 adotta 
i suggerimenti del sistema 1 senza modificarli ma quando compaiono difficoltà interviene e procede ad una elaborazione dettagliata per risolvere il problema contingente. ${ }^{8} \mathrm{Si}$ connota una relazione continua tra il sistema 1 presenzialista, sempre attivo (non può essere disattivato a piacere) ed il sistema 2 che compie continuamente sforzi per entrare in scena.

Considerato quindi il ruolo del sistema 1 nelle scelte effettuate in qualunque ambito della nostra vita è importante riassumerne le principali caratteristiche: ${ }^{8}$

Trascura ambiguità e dubbi: i) inferisce e inventa cause e intenzioni; ii) tende in partenza a credere e a confermare; iii) esagera su aspetti di coerenza emozionale; iv) si concentra su prove esistenti e tende a ignorare le prove mancanti; v) fornisce un peso eccessivo a eventi a bassa probabilità; vi) utilizza le scorciatoie mentali del pensiero euristico.

\section{Processo di decision making: fast or slow?}

Il modello a doppio sistema descritto si applica anche al processo di decision making clinico del medico.$^{10}$ In tale contesto il sistema 1 agisce in una prima fase, dopo la raccolta delle informazioni anamnestiche (data gathering), con un percorso veloce e non analitico che abbina i dati del paziente con un approccio diagnostico basato su pattern di riconoscimento e schemi mentali del medico (Tabella 1) generando un'ipotesi diagnostica (hypothesis generation).

La seconda fase, è invece fondamentale per la valutazione delle ipotesi (hypothesis evaluation). Il processo è sostenuto dal sistema 2, più lento ed in grado di generare una complessa fase di elaborazione delle informazioni necessaria per una corretta diagnosi differenziale sulla base delle ipotesi prodotte nella fase iniziale.

L'interazione dei sistemi 1 e 2 può essere interrotta dal medico per vari motivi, limitando in tal caso, l'analisi delle informazioni disponibili. Ad esempio si può essere soddisfatti dal riconoscimento di un pattern diagnostico, dagli esiti di esami di laboratorio o radiologici, anche se non completamente congrui con la storia clinica del paziente, con il conseguente rischio di effettuare una diagnosi prematura. Questo è un approccio tipico del pensiero euristico condizionato da pregiudizi cognitivi, numerosi e non facilmente identificabili,${ }^{10}$ che vedremo nel capitolo successivo. In sostanza il sistema 1 tende ad accettare la conclusione clinica più coerente ma non tiene conto delle informazioni non disponibili. Ne consegue che tale metodo è utile a clinici esperti, in modo particolare per la formulazione di diagnosi rapide, ma espone tutti medici a maggiori rischi di errori diagnostici. ${ }^{5,10}$

\section{Pensiero euristico e bias}

L'aspetto essenziale del pensiero euristico e dei bias è quindi quello di sostituire a un giudizio difficile un giudizio più facile. Un lungo elenco di pregiudizi di carattere clinico, descritti in sintesi nella Tabella 2, è stato proposto da Croskerry ${ }^{11}$ che ne ha analizzato anche il ruolo peculiare nell'ambito di un sistema di emergenza-urgenza caratterizzato da scenari clinici molto incerti e tempi decisionali ristretti. ${ }^{12}$

Tabella 1. Esempi di approccio diagnostico. ${ }^{10}$

\begin{tabular}{ll}
\hline Modelli & Descrizione \\
\hline Pattern di riconoscimento & Esempi di schemi familiari: \\
& a) cefalea, rigidità al collo, fotofobia, febbre $>$ MENINGITE \\
& b) dolore addominale in sede tipica, febbre, insorgenza recente $>$ APPENDICITE \\
& Vantaggi: \\
& $-\quad$ diagnosi rapida \\
& Limiti: \\
& $-\quad$ difficoltà diagnostica se lo schema non presenta tutti gli elementi tipici del pattern \\
& $-\quad$ utile al clinico esperto \\
& $-\quad$ necessità di conferma del pattern per ridurre gli errori \\
\hline Strategia di esclusione degli scenari più critici & Approccio utile per escludere le diagnosi più critiche per il paziente \\
& Esempio: dolore toracico acuto \\
& Vantaggi: \\
& $-\quad$ consente di evidenziare diagnosi life saving \\
& Limiti \\
& $-\quad$ non vengono effettuate ulteriori ipotesi diagnostiche oltre a quelle life saving
\end{tabular}


Possiamo comunque sintetizzare in poche semplici regole l'attività del pensiero euristico: i) la soluzione più semplice a un problema clinico è spesso corretta; ii) è molto probabile l'utilizzo della esperienza clinica recente per gestire quella successiva iii) le diagnosi 'ovvie' spiegano più spesso i sintomi di quelle 'non ovvie'.

Esistono inoltre condizioni che limitano ulteriormente il pensiero analitico: i) ambientali o situazionali: operare in condizioni di emergenzaurgenza o con ridotte risorse disponibili. In tali contesti è difficile effettuare una completa raccolta delle informazioni anamnestiche per la gestione di una caso clinico. Siamo quindi esposti a ritenere più probabili le diagnosi mentalmente disponibili che di solito corrispondono a quelle che più comunemente osserviamo (bias di disponibilità); ii) personali: eccesso di fiducia nelle proprie capacità, spinte emozionali o di risultato. Condizioni che inducono ad agire con informazioni incomplete effettuando scelte basate più sulle opinioni invece che sulle evidenze disponibili (bias di conclusione prematura di un processo diagnostico); iii) culturali: la mancanza di adeguate nozioni di statistica può favorire diagnosi errate. Ad esempio siamo indebitamente influenzati dalle caratteristiche degli eventi precedenti piuttosto che dalla probabilità che questi accadano. Tale eventualità descrive la fallacia dello scommettitore un errore logico che riguarda l'errata convinzione che eventi occorsi nel passato influiscano su quelli futuri nell'ambito di attività governate dal caso. Tipica è l'erronea convinzione che un evento casuale ha meno probabilità di verificarsi perché accaduto di recente. Questo limite culturale se applicato ad un processo decisionale di carattere clinico può generare, ad esempio, la seguente conclusione: oggi ho già diagnosticato quattro emorragie cerebrali quindi l'attuale paziente con mal di testa può avere solo una cefalea.

\section{Giudizio intuitivo: \\ più competente o più consapevole?}

A questo punto è naturale porci alcune domande: quando ci si può fidare di un professionista che afferma di avere una intuizione? Quando i giudizi riflettono vera competenza e quando invece rivelano una illusione di validità? Kahneman ${ }^{8}$ afferma che se sono presenti due condizioni: un ambiente abbastanza regolare da essere prevedibile ed esiste l'opportunità di imparare con regolarità ed effettuare una pratica prolungata, è probabile che le valutazioni siano competenti e quindi affidabili. In sostanza come ritengono altri autori, il miglioramento della expertise del professionista unitamente al continuo aggiornamento delle nozioni mediche appaiono gli unici strumenti utili per perfezionare la capacità di giudizio. Tali aspetti potrebbero comunque non essere sufficienti per limitare l'impatto dei biases cognitivi mentre acquisire maggiore consapevolezza dei limiti del nostro pensiero sembra un utile presupposto per raggiungere tale scopo. ${ }^{10-13}$

\section{Metacognizione e capacità di decision making}

La Metacognizione, definita ${ }^{6}$ come la capacità di riflettere sul proprio pensiero (thinking about thinking), si pone tra le strategie utili per comprendere il pensiero alla base del ragionamento clinico e dell'attività decisionale. ${ }^{13-17}$ I processi metacognitivi dipendono da una complessa interazione di regioni cerebrali responsabili del livello di attenzione per lo svolgimento di un compito, valutano i requisiti di un problema, pianificano le strategie di soluzione e ne monitorizzano i progressi modificando il piano quando necessario. Inoltre da essi dipendono la consapevolezza di sé, della memoria e persino delle aspettative individuali. ${ }^{6}$ Le strategie metacognitive hanno dimostrato di avere un impatto positivo sull'apprendimento e sulle prestazioni all'interno di contesti educativi non medici (matematica, lettura, scrittura), specialmente per migliorare le capacità di problem solving, ${ }^{18,19}$ mentre hanno trovato poca attenzione da parte della letteratura dedicata ai percorsi di apprendimento rivolti al personale sanitario. ${ }^{17}$ Solo alcuni studi hanno osservato una correlazione tra metacognizione ed il miglioramento del ragionamento clinico nel personale infermieristico e tra medici pediatri. ${ }^{19,20}$

In ambito clinico appare invece evidente la relazione tra pregiudizi cognitivi ed errori diagnostici. ${ }^{21-24}$ Uno studio focalizzato sugli errori nel processo di decision making commessi da medici internisti ha evidenziato una maggiore accuratezza diagnostica, statisticamente significativa, quando erano utilizzate competenze metacognitive rispetto a quando venivano impiegati metodi di ragionamento non analitici. ${ }^{22}$ Attualmente in diversi paesi, tra cui USA e Gran Bretagna, alcune agenzie di accreditamento richiedono ai medici in formazione capacità di autovalutazione basate sul riconoscimento dei propri punti di forza e di debolezza come parte di un percorso di apprendimento e miglioramento delle competenze. ${ }^{6,13}$ 


\section{Tecniche per migliorare le capacità metacognitive}

Verranno ora esposte in sintesi alcune tecniche ritenute utili a sviluppare capacità metacognitive in grado di migliorare processi di apprendimento e appropriatezza del giudizio clinico.

\section{Riflessione}

Caratteristica di questa tecnica è l'analisi delle azioni attraverso l'abitudine di pensare agli esiti e conseguenze delle nostre scelte nella pratica clinica quotidiana. Tale strumento appare particolarmente utile per stimolare gli studenti (in particolare quelli meno dediti al ragionamento analitico) a non accettare la diagnosi che più facilmente viene evocata. Appare inoltre importante durante riunioni di de-briefing o nel corso di incontri dove si analizzano casi clinici utili per identificare criticità organizzative. Una tipica domanda che potrebbe essere evocata da questa tecnica è: si sarebbero potute intraprendere scelte con esiti più favorevoli?

\section{Organizzatori grafici}

Permettono rappresentazioni visive di dati e process $\mathrm{i}^{25}$ favorendone la comprensione e memorizzazione. ${ }^{26}$ Vengono utilizzati anche in ambito didattico: l'uso di immagini consente di sintetizzare concetti, procedure e risultati. ${ }^{27}$ Gli organizzatori grafici possono anche essere impiegati per fornire indicazioni generali, piani d'azione e feedback agendo come strumento metacognitivo favorente la presa di coscienza delle informazioni disponibili.

Tabella 2. Comuni biases cognitivi. ${ }^{12}$

\begin{tabular}{ll}
\hline Bias & Descrizione \\
\hline Ancoraggio & Tendenza a fissarsi troppo precocemente su alcune caratteristiche specifiche (definite ancore) del quadro \\
clinico per cui il successivo processo decisionale ne sarà fortemente influenzato. Questo approccio può & essere utile (consente un rapido inquadramento del caso clinico) ma alcuni medici risentono eccessivamente \\
& di tale effetto, non riescono a modularlo ed eventuali informazioni che vengono acquisite nelle fasi successive \\
& del processo diagnostico non pongono in discussione quelle ottenute nella fase iniziale. Gli effetti delle \\
& ancore possono essere minacciosi perché non sappiamo come esse ci guidano, limitano il nostro pensiero e \\
& non riusciamo a immaginare cosa avremmo pensato se l'ancora fosse stata diversa.
\end{tabular}

Accertamento

Si verifica quando l'approccio cognitivo del medico è preformato su ciò che si aspetta specificamente di trovare. Ad esempio il clinico ha maggiori probabilità di porre diagnosi di insufficienza cardiaca congestizia in un paziente che riferisce di non avere assunto regolarmente la terapia diuretica.

Disponibilità

Tendenza a giudicare la frequenza degli eventi in base alla facilità con cui gli esempi ci vengono in mente. ${ }^{8}$ Di conseguenza le esperienze comuni saranno quelle più agevolmente ricordate. L'euristica è guidata dal presupposto che 1'evidenza più disponibile sia la più pertinente. Pertanto, se ad esempio un medico di emergenza ha visitato recentemente un paziente con cellulite che ha determinato uno quadro di shock settico (e un ricovero in terapia intensiva), avrà una maggiore tendenza a ricordare la sepsi quando si presenterà un altro paziente con cellulite.

Conferma

Basato sulla generale tendenza degli individui a ricercare prove di conferma di un'ipotesi piuttosto che verificare informazioni che tentano di confutarla. Le conseguenze sono che un medico può formulare una diagnosi attraverso dati clinici relativamente deboli o ambigui ignorandone altri che tendono a smentirla.

Framing o effetti di formulazione Modi diversi per presentare le stesse informazioni suscitano percezioni (anche emozionali) e possibili ricadute diagnostiche differenti. Ad esempio: descrizioni dello stesso scenario clinico come possibile sepsi o cellulite in una giovane persona per il resto sana possono incidere sulle scelte gestionali: nel primo caso si può essere inclini ad accertamenti approfonditi ed al ricovero ospedaliero mentre nel secondo più favorevoli ad un approccio terapeutico domiciliare.

Conclusione prematura

Nella valutazione iniziale di un caso clinico si possono effettuare diverse ipotesi diagnostiche. La conclusione prematura si verifica quando una diagnosi viene avvalorata prima che sia stata completamente verificata. Questo può riflettere una certa pigrizia di pensiero e il desiderio di giungere rapidamente a una definizione del quadro clinico. Condizioni di affaticamento o stress possono favorire questo tipo di bias.

Rappresentatività

Compare quando ci si concentra sulla somiglianza tra descrizione e lo stereotipo cognitivo, ignorando i dubbi in merito alla veridicità della descrizione (8). Pertanto può accadere che il quadro clinico di un paziente sia correlato a modelli mentali del medico (tradizionalmente nel percorso formativo del medico si insegna il riconoscimento di pattern diagnostici) che per la loro rappresentatività impediscono di valutare altre opzioni diagnostiche atipiche. 


\section{Feedback}

La capacità di autovalutazione delle nostre prestazioni è un processo metacognivo complesso in particolare per gli studenti la cui crescita formativa trae beneficio da feedback oggettivi ed attendibili che in genere vengono raccomandati precocemente nel corso di attvità professionali. Archer ha osservato invece che feedback tardivi risultano più utili ed efficaci per migliorare processi di apprendimento. ${ }^{28}$

\section{Think-aloud strategies}

Tecnica frequentemente utilizzata per l'insegnamento in ambito sanitario. Consente di ragionare ad alta voce sui processi decisionali e motivazioni responsabili di conclusioni diagnostiche. Risulta particolarmente utile a tutor o insegnanti per analizzare e condividere con gli studenti il pensiero di un professionista esperto: come risolve quesiti clinici ed esprime giudizi. ${ }^{29}$

\section{Cognitive debiasing and forcing strategies}

L'obiettivo è quello di forzare gli studenti a limitare l'eccessiva fiducia delle proprie convinzioni e ridurre l'effetto di bias cognitivi chiedendo di produrre argomentazioni a favore e contro ipotesi o conclusioni diagnostiche. Anche la riflessione, come indicato in precedenza, è di fatto una tecnica di debiasing in grado di produrre buoni risultati. ${ }^{14} \mathrm{I}$ medici esperti utilizzano in modo automatico tecniche di forzatura cognitiva basate sul principio di stepping back che consentono mentalmente di rivedere il caso con un approccio olistico analizzando le principali insidie e criticità prima di procedere con ulteriori scelte cliniche. Tale tecnica può essere insegnata agli studenti per migliorare le loro capacità di decision making e ridurre errori diagnostici. ${ }^{30-32}$

\section{Predizione del risultati}

Metodo di particolare utilità per lo studente che attraverso l'analisi di precedenti performance è in grado di pianificare strategie efficaci per migliorare gli esiti di prestazioni future. Ad esempio si possono valutare i punti di forza e di debolezza di pregresse attività professionali o didattiche (esiti di esami universitari), argomenti sui quali si sono incontrate le maggiori difficoltà e quindi pianificare azioni correttive. Tale approccio consente anche di determinare se le proprie conoscenze soddisfano una determinata soglia di competenza e identificare risorse, tecniche e metodi specifici utili per colmare le eventuali lacune..$^{33}$

\section{Self-questioning}

Tecnica metacognitiva che comporta una serie di specifiche domande verbali o scritte rivolte a definire nel dettaglio gli aspetti diagnostici e terapeutici di un quadro clinico. L'esito può tradursi in ulteriori riflessioni, un nuovo approccio mentale al problema ed autocorrezione di alcune scelte. La maggiore difficoltà nella applicazione pratica di questa strategia è porre le domande idonee per autovalutare i maggiori problemi di quel paziente Alcuni autori ${ }^{34}$ hanno creato delle Question Card per aiutare i medici meno esperti o gli studenti nel formulare tali quesiti.

\section{Limiti della metacognizione nella pratica clinica}

Uno dei limiti principali limiti delle tecniche metacognitive è rappresentato dalla mancanza di condivisione sulla modalità più efficace per l'applicazione pratica in ambito clinico. ${ }^{35,36}$ Questa criticità è particolarmente evidente quando il processo decisionale è svolto in ambienti o situazioni stressanti. In tali contesti i medici devono prendere decisioni rapide basate su intuito ed esperienza facendo leva sull'attività di pensiero del sistema 1 . Paradossalmente le tecniche metacognitive richiedono tempo e rallentano l'intero processo decisionale quindi risultando poco efficaci per ridurre bias ed errori. ${ }^{37}$ Recentemente alcuni studi, ${ }^{5}$ ancora con casistiche di piccole dimensioni, hanno proposto l'applicazione di checklist mnemoniche in grado di facilitare l'applicazione delle tecniche metacognitive che sembrano risultare efficaci anche nella real life dei setting clinici. Saranno necessari trial più ampi per avere le conferme di questi risultati preliminari.

\section{Conclusioni}

La diagnosi è una competenza peculiare della professione medica che trova nelle capacità cognitive del pensiero umano e negli attuali contesti clinici sempre più complessi i maggiori ostacoli, quasi a raffigurare un collo di bottiglia limitante un appropriato giudizio clinico (Figura 1). Il processo decisionale è svolto prevalentemente, secondo le più recenti teorie, con modalità intuitiva e ampio ricorso alla memoria associativa (sistema 1) ma influenzato da bias cognitivi di cui solo in parte il medico è consapevole. Il continuo aggiornamento basato sulla acquisizione di nozioni non è l'unico elemento in grado di completare il curriculum del professionista la cui expertise dovrà verosimilmente arricchirsi, nei 


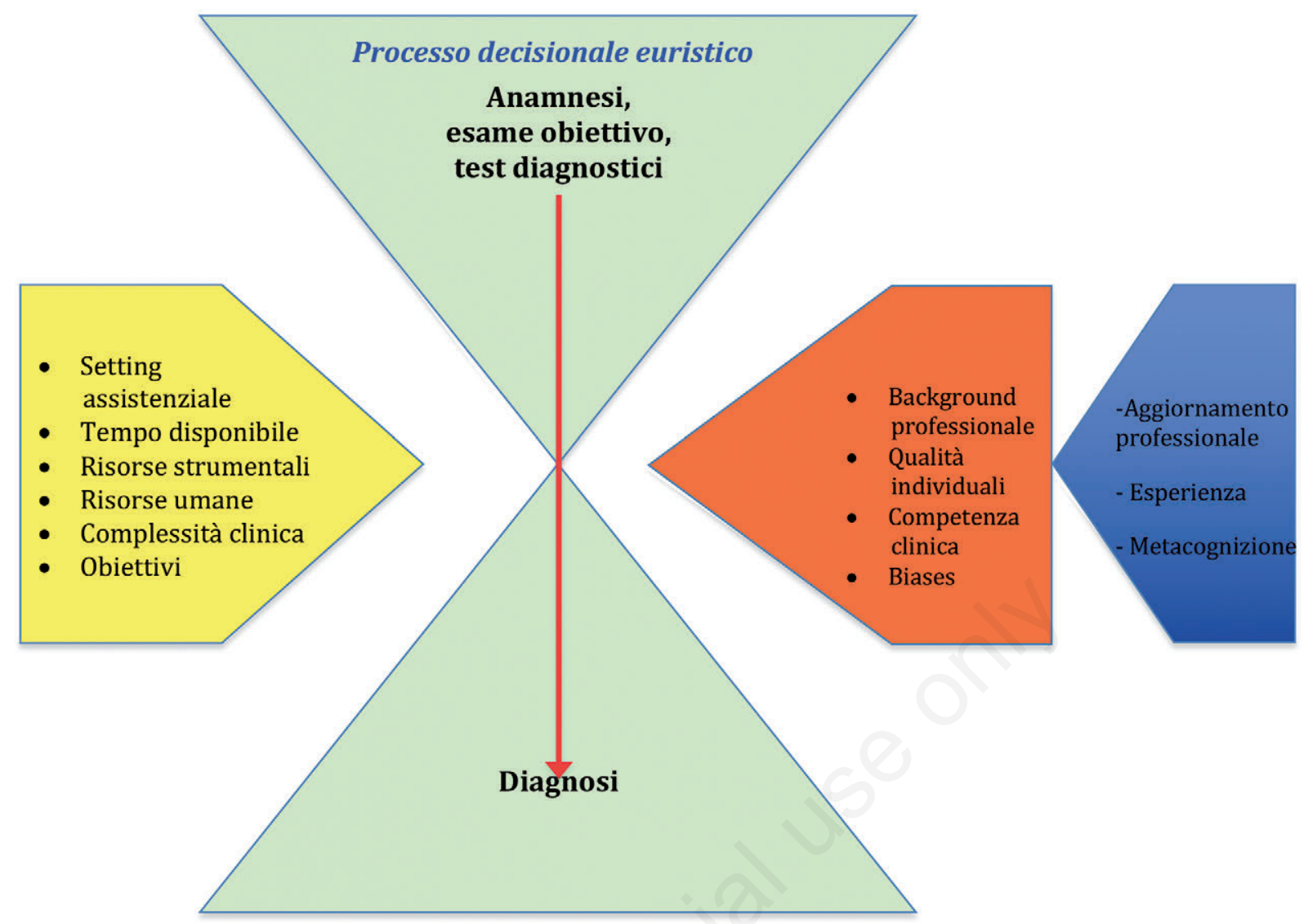

Figura 1. "Collo di bottiglia" del giudizio clinico: elementi condizionanti.

prossimi anni, di strumenti cognitivi utili per rendere più appropriati i propri giudizi clinici nei diversi setting assistenziali (emergenza-urgenza, specialità mediche e chirurgiche). La metacognizione gioca un ruolo importante sia per favorire il percorso di apprendimento dello studente che per migliorare le capacità diagnostiche del medico esperto attraverso l'acquisizione di una maggiore coscienza dei propri limiti e mediante l'uso di tecniche utili per limitare l'effetto dei bias.

La raccomandazione sia per le Facoltà di Medicina e Chirurgia che per le società scientifiche mediche è quella di iniziare a discutere di tali aspetti che, alla luce delle attuali conoscenze, sono fattori condizionanti la qualità della prestazioni mediche.

\section{Punti chiave}

- Alcuni errori diagnostici in ambito clinico sono determinati da processi decisionali condizionati da cognitive bias.

- La metacognizione è in grado di migliorare le capacità di decisione making ma la ricerca delle migliori modalità per l'applicazione nella pratica clinica ha trovato poco spazio nelle letteratura medica e comunque è ancora raramente oggetto di insegnamento e confronto.

- Tecniche utili per sviluppare processi metacognitivi includono la riflessione, feedback, tecniche di de-biasing, confronti ad alta voce, metodi di self-questioning.

- Studenti e medici dovrebbero costantemente riflettere sui personali punti di forza e di debolezza come parte essenziale di un continuo processo di miglioramento della proprie competenze.

\section{Bibliografia}

1. Kohn L, Corrigan J, Donaldson M (Eds.) To err is human: building a safer health system. Washington, D.C.: National Academy Press, 2000.

2. Kuhn GJ. Diagnostic errors. Acad Emerg Med. 2002;9:740-750. 
3. Leape L, Brennan T, Laird N et al. The nature of adverse events in hospitalized patients - results of the Harvard Medical Practice Study II. N Engl J Med 1991; 324; 377-84.

4. Gallagher EJ. Ann Emerg Med 2003; 41:121-122.

5. Chew KS et al. Teaching metacognition in clinical decision-making using a novel mnemonic checklist: an exploratory study, Singapore Med J 2016, 57 (12), 694-700.

6 Colbert CY et al. Teaching Metacognitive Skills: Helping Your Physician Trainees in the Quest to 'Know What They Don't Know' The American Journal of Medicine, vol 128, No 3, March 2015.

7. Gladwell M. Blink: the power of thinking without thinking . London: Little, Brown and Company, 2005.

8. Kahneman D. Thinking, fast and slow. London: Penguin Books, 2012.

9. Stanovich KE, West RF. Individual Differences in Reasoning: Implication for the Rationality Debate, in «Behavioral and Brain Sciences», 23, 2000, pp. 645-665.

10. Trimble M, Hamilton P. The thinking doctor: clinical decision making in contemporary medicine, Clinical Medicine 2016 Vol 16, No 4: 343-6.

11. Croskerry P. Achieving quality in clinical decision making: cognitive strategies and detection of bias . Acad Emerg Med 2002; 9; 1184-204.

12. Keijzers $G$ et al. Deliberate clinical inertia: Using meta-cognition to improve decision-making Emergency - Medicine Australasia 2018, 30, 585-590.

13. Marcum JA. An integrated model of clinical reasoning: dual process theory of cognition and metacognition. $\mathrm{J}$ Eval Clin Pract. 2012;18:954-961.

14. Mamede S, van Gog T, van den Berge K, et al. Effect of availability bias and reflective reasoning on diagnostic accuracy among internal medicine residents. JAMA. 2010;304:1198-1203.

15. Cutrer WB, Sullivan WM, Fleming AE. Educational strategies for improving clinical reasoning. Curr Probl Pediatr Adolesc Health Care. 2013;43:248-257.

16. Croskerry P, Norman G. Overconfidence in clinical decision making. Am J Med. 2008;121:S24-S29.

17. Burman NJ, Boscardin CK, Van Schaik SM. Careerlong learning: relationship between cognitive and metacognitive skills. Med Teach. 2014;36:715-723.

18. Pintrich PR, Wolters CA, Baxter GP. Assessing metacognition and self-regulated learning. In: Schraw $\mathrm{G}$, Impara JC, eds. Issues in the Measurement of Metacognition. Lincoln, NE: Buros Institute of Mental Measurements; 2000. Available at: http:// digitalcommons.unl.edu/burosmetacognition/3/.

Accessed December 12, 2013.

19. Azevedo R. Theoretical, conceptual, methodological and instructional issues in research on metacognition and self-regulated learning: a discussion. Metacog Learn. 2009;4:87-95.

20. Kuiper RA, Pesut DJ. Promoting cognitive and metacognitive reflective reasoning skills in nursing practice: self-regulated learning theory. J Adv Nurs. 2004;45:381-391.

21. Monteiro S, Sherbino J, Sibbald M, Norman G. Critical thinking, biases and dual processing: The enduring myth of generalisable skills Medical Education. 2019;00:1-8.

22. Mamede S, van Gog T, van den Berge K, et al. Effect of availability bias and reflective reasoning on diagnostic accuracy among internal medicine residents. JAMA. 2010;304:1198-1203.

23. Croskerry P. The importance of cognitive errors in diagnosis and strategies to minimize them. Acad Med. 2003;78:755-780.

24. Dunphy BC, Cantwell R, Bourke S, et al. Cognitive elements in clinical decision-making: toward a cognitive model for medical education and understanding clinical reasoning. Adv Health SciEduc Theory Pract. 2010;15:229-250.

25. Dye GA. Graphic organizers to the rescue! Helping students link and remember information. Teach Excep Children. 2000;32:72-76.

26. Torre DM, Dalley B, Stark-Schweitzer T, Siddartha S, Petkiva J, Ziebert M. A qualitative evaluation of medical student learning with concept maps. Med Teach. 2007;29:949-955.

27. West DC, Park JK, Pomeroy JR, Sandoval J. Concept mapping assessment in medical education: a comparison of two scoring systems. Med Educ. 2002; 36:820-826.

28. Archer JC. State of the science in health professional education: effective feedback. Med Educ. 2010;44: 101-108.

29. Lajoie SP. Metacognition, self regulation, and selfregulated learning: a rose by any other name? Educ Psychol Rev. 2008;20:469-475.

30. Finn B. Framing effects on metacognitive monitoring and control. Mem Cognit. 2008;36:813-821.

31. Bond WF, Deitrick LM, Arnold DC, et al. Using simulation to instruct emergency medicine residents in cognitive forcing strategies. Acad Med. 2004;79:438-446.

32. Croskerry P. Cognitive forcing strategies in clinical decision making. Ann Emerg Med. 2003;41:110-120.

33. Niedringhaus K. Teaching Better Research Skills by Teaching Metacognitive Ability. Perspectives: Teaching Legal Research and Writing. 2010;18:113. Available at: http://info.legalsolutions. thomsonreuters. com/signup/ newsletters/9.aspx. Accessed December 15,2014

34. Pangaro L. Uniformed Services University Medicine Clerkship Handbook. 2013. Bethesda, MD: Uniformed 
Services University. Available at: http://www.usuhs. mil/med/clerkship/index.html. Accessed July 30, 2014.

35. Croskerry P, Singhal G, Mamede S. Cognitive debiasing 1: origins of bias and theory of debiasing. BMJ Qual Saf 2013; 22 Suppl 2:ii58-ii64.
36. Croskerry P. From mindless to mindful practicecognitive bias and clinical decision making. N Engl J Med 2013; 368:2445-8.

37. Norman G. Dual processing and diagnostic errors. Adv Health Sci Educ Theory Pract 2009; 14 Suppl 1:37-49. 


\title{
Complessità, caos e ragionamento clinico
}

\author{
Flavio Tangianu, ${ }^{1}$ Salvatore Corrao, ${ }^{2}$ Antonino Mazzone, ${ }^{3}$ Paola Gnerre, ${ }^{4}$ Giovanni Scanelli, ${ }^{5}$ Maurizia Gambacorta, ${ }^{6}$ \\ Daniela Tirotta, ${ }^{7}$ Andrea Fontanella, ${ }^{8}$ Michele Adamo, ${ }^{9}$ Roberto Nardi ${ }^{10}$ \\ ${ }^{1}$ Dipartimento di Medicina, Università di Insubria (VA); ${ }^{2}$ Dipartimento di Medicina Clinica, ARNAS Civico Di Cristina \\ Benfratelli, Palermo; PROMISE, Università di Palermo; ${ }^{3}$ Medicina Interna, Legnano (MI); ${ }^{4}$ Medicina Interna 2, Ospedale San

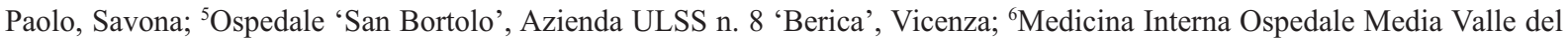 \\ Tevere, ASL 1 Umbria; ${ }^{7}$ Medicina Interna, Cattolica (RN); ${ }^{8}$ Medicina Interna, Ospedale del Buon Consiglio-Fatebenefratelli, \\ Napoli; ${ }^{9}$ Medicina Generale e Cardiologia extra-ospedaliera, Bologna; ${ }^{10}$ Medicina Interna, Bologna, Italia
}

\section{Cosa intendiamo per complessità in Medicina}

La complessità non è sinonimo di complicato o di confuso: essa è riferita all'organizzazione di un sistema, con molteplici entità integrate fra loro e reciprocamente interagenti in equilibrio dinamico. ${ }^{1} \mathrm{Al}$ contrario, in un sistema complicato si conoscono le proprietà di ogni singolo componente, le regole delle interazioni esistenti e ne è noto l' esito atteso finale, come un enorme 'puzzle'. Definire il concetto di complessità non è semplice. ${ }^{2}$ 'The Complexity of Measuring Clinical Complexity' è il titolo di un editoriale pubblicato sugli Annals of Internal Medicine nel 2011. ${ }^{3}$ Secondo il Ministero della Salute la 'complessità in medicina' considera l'insieme delle diverse condizioni morbose non solo in quanto compresenti, ma nella loro interazione multidimensionale (comorbilità, multi-morbilità a genesi comune o diversa, convergenza su elementi clinici comuni e interconnessione con acuzie e cronicità e con l'intensità di cura necessaria); la 'medicina della complessità' si esercita con la sintesi, che diviene sinergia, tra l'EBM e la medicina narrativa, cogliendo attraverso tutti i sensi ogni dinamica della vita dell'individuo. ${ }^{4}$ In natura esistono tre diverse classi di sistemi dinamici, riscontrabili in biologia, in medicina, psicologia, in economia e nelle scienze politiche, ${ }^{5}$ ben descritti da Weaver nel 1948: ${ }^{6}$ i) i «sistemi semplici», caratterizzati dalla presenza di poche variabili; ii) i «sistemi a complessità disorganizzata», caratterizzati da un numero

Corrispondente: Roberto Nardi, Medicina Interna, Bologna, Italia.

E-mail: nardidoc48@gmail.com

Articolo pubblicato secondo la Creative Commons Attribution NonCommercial 4.0 License (CC BY-NC 4.0).

${ }^{\circ}$ Copyright: the Author(s), 2020

Licensee PAGEPress, Italy

QUADERNI - Italian Journal of Medicine 2020; 8(1):81-90 estremamente elevato di variabili, ciascuna con un comportamento individuale casuale o sconosciuto; iii) i «sistemi a complessità organizzata», caratterizzati da un numero considerevole di variabili connesse in un tutto organico. Lo studio dei sistemi complessi riguarda le proprietà collettive di un contesto contrassegnato da un gran numero di componenti in interazione tra loro, con risultati osservabili diversi dalla mera somma delle parti. La scienza della complessità tende a comprendere l'architettura generale delle variabili in causa, focalizzandosi sulla struttura delle interconnessioni esistenti piuttosto che sulle singole componenti. ${ }^{7,8}$ La strutturazione delle relazioni tra diversi fattori è stata definita dal concetto di Gestalt (letteralmente: 'ciò che compare agli occhi', ovvero la 'forma percepita'), inteso come la 'visione d'insieme', in base alla capacità di valutare la 'complessità' in quanto tale, non spezzettata né ridotta in singoli elementi, ${ }^{9}$ dato che 'Il tutto è diverso dalla somma delle sue parti' [Das Ganze unterscheidet sich von der Summe seiner Teile $].{ }^{10}$ La Gestal è il motore fondamentale della 'saggezza' (pagina 85): essa caratterizza '- al di là della conoscenza posseduta - i 'professionisti decisionali' rispetto a quelli 'incerti', dato che i fattori confondenti nelle scelte da effettuare sono numerosi, le decisioni devono essere assunte in contingenze di tempo limitato e di informazioni incerte, con conseguente possibilità di errore. La valutazione della complessità dei pazienti consente di ottenere un approccio mirato ai bisogni specifici del paziente, attraverso un'adeguata gerarchizzazione degli stessi. L'introduzione di elementi di valutazione clinica basata sul 'Comprehensive Assessment' in aggiunta a meri elementi di tipo statistico consente altresì di ottenere diversi vantaggi: ad esempio, per: i) una migliore definizione delle caratteristiche delle popolazioni oggetti di studio; ii) la stratificazione prognostica nel singolo paziente; iii) la disponibilità di ulteriori elemento di giudizio clinico ai fini del decision making; iv) la disponibilità di ulteriori elementi di giudizio sulle possibilità di allocazione delle risorse e della gestione delle informazioni (Tabella 1). 


\section{Cosa intendiamo per paziente complesso in Medicina Interna?}

La malattia non è solamente uno stato patologico oggettivabile, misurabile, classificabile, ma anche malessere e sofferenza soggettiva, spesso espressione di un rapporto tra individuo e ambiente,${ }^{11}$ come postulato dalla «teoria del campo» di Kurt Lewin, ${ }^{12}$ per cui il 'sistema complessità' non è che la funzione tra numero di relazioni e l'ambiente che lo circonda. Non esiste una definizione univoca di complessità in Medicina Interna. ${ }^{13}$ Complessità è un insieme di elementi uniti da un intreccio di relazioni, capaci di interagire tra loro e di adattarsi alle modifiche ambientali Studiare la complessità significa comprendere il bisogno di ricomposizione, di riaggregazione delle conoscenze, superando la frammentarietà. ${ }^{14} \mathrm{La}$ complessità si caratterizza per il gran numero di variabili, con dati forniti spesso in modo impreciso. ${ }^{15}$ Secondo la definizione della Facoltà di Sociologia dell'Università di Vienna la complessità è l'embricarsi delle influenze di due o più componenti nello stesso individuo, ad esempio: malattie della sfera fisico-psichica, contesto sociale ed economico, farmaci assunti. La definizione di 'paziente complessò', adottata dall'Agency for Healthcare Research and Quality (AHRQ), si riferisce a una persona affetta da due o più malattie croniche, in cui ciascuna delle condizioni morbose presenti $e^{\prime}$ in grado d'influenzare l'esito delle cure delle altre coesistenti, attraverso varie modalità: la limitazione della speranza di vita, l'aumentata morbilità intercorrente, le interazioni tra terapie farmacologiche, l'impossibilita` del pieno impiego di cure adeguate per controindicazione $o$ assenza di evidenze, eccetera. ${ }^{16}$ Nell'accezione più completa di complessità, le componenti di tipo biologico, socioeconomico, culturale, comportamentale e ambientale diventano tutte importanti determinanti di salute, ${ }^{17}$ purtroppo - e paradossalmente - considerate, nei criteri di eleggibilità, come potenziali 'confondenti' ai fini di un' 'oggettiva' valu- tazione dei risultati di un trial clinico. Complessità delle cure può essere intesa anche come una maggiore quantità di tempo da dedicare al paziente per valutare ed erogare l'assistenza necessaria. ${ }^{18}$ In Medicina Interna la gestione di un paziente complesso significa possedere competenze peculiari per affrontare non solo le diverse co-morbilità, ma tutte le sfide insite nel singolo caso. ${ }^{19} \mathrm{Al}$ contrario della sub-specializzazione, generalmente riferita ad un singolo organo o apparato e di cui si riconosce agevolmente l'ambito di competenza, l'internista è il 'medico dell'integrazione', concetto non immediatamente comprensibile. Egli dovrebbe infatti occuparsi specificamente della persona nella sua globalità, coniugando la cultura della 'inter-poli-trans-disciplinarietà' con quella della 'complessità', per la gestione di patologie complesse e di tutti gli elementi contestuali. ${ }^{20} \mathrm{La}$ complessità pone all'internista obiettivi non solo di miglioramento della sua competenza, ma anche della capacità di coordinamento dell'azione medica polispecialistica e della sua adattabilità al cambiamento e all'incertezza, attraverso nuove conoscenze e con i massimi livelli di consapevolezza in relazione al contesto (situation awareness), ai fini della decisione più adeguata. ${ }^{21}$ In questo ambito una delle competenze specifiche da sviluppare è la comunicazione con gli altri specialisti nel momento della consulenza che non può essere mai un atto a se stante o burocratico, ma che deve essere una interazione attiva con chi governa il processo diagnostico-terapeutico dello specifico caso clinico.

\section{Il paziente complesso è realmente esclusivo della Medicina Interna?}

La complessità e la non linearità sono molto diffuse in ambito bio-medico, in relazione a fenomeni che interagiscono fra loro in un contesto mutevole. ${ }^{22}$ Da molto tempo noi Internisti sosteniamo che la Medicina Interna è la Medicina della complessità, ${ }^{11,17,23}$ insieme ai Colleghi geriatri. ${ }^{24}$ Molti altri specialisti di

Tabella 1. Implicazioni pratiche correlate alla gestione della complessità del paziente internistico.

- Necessità di riconoscere e rispondere a situazioni correlate alla complessità, all'incertezza e all'ambiguità della pratica medica;

- Necessità di una valutazione multi-dimensionale del singolo paziente;

- Necessità di ricercare sistematicamente le co-morbilità sottese (ad iceberg) o manifeste ed associate (clustering disease);

- Necessità di individuare i pazienti fragili o con deficit funzionali;

- Necessità di garantire la 'gerarchizzazione delle priorità' nel contesto dei diversi problemi in causa;

- Necessità di una definizione prognostica anche i fini delle decisioni da assumere;

- Necessità di una buona capacità di comunicazione ed inter-relazione col paziente fragile e/o complesso e con i suoi familiari;

- Necessità di selezionare i trattamenti indispensabili, mediante una gerarchizzazione delle priorità, tenendo conto dell'urgenza clinica, delle valutazioni prognostiche e delle risorse disponibili;

- Necessità di definire un piano di gestione ed un trattamento mirato sul singolo paziente ('tailored therapy' e 'tailored follow-up');

- Necessità di garantire il coordinamento delle cure e la prevenzione delle riacutizzazioni delle malattie croniche;

- Necessità di considerare l'elevato rischio di danno iatrogenico (politerapia, gestione frammentata dell'assistenza, fornita da più specialisti, interazioni farmacologiche, ADR, incompatibilità, controindicazioni);

- Necessità di risk management, correlato all'incertezza implicita e all'aumentato rischio di errore. 
area medica, anche alla luce dei dati demografici, rivendicano che la complessità dei pazienti riguarda tutte le specializzazioni mediche che si occupano di pazienti anziani, con co-morbilità multiple, sia per le loro caratteristiche cliniche, che per la complessità dell'organizzazione del Sistema sanitario. ${ }^{9,25-30}$ Nonostante molti successi, la medicina non è riuscita a risolvere una lunga lista di condizioni comuni. Questo fallimento può derivare da una riluttanza a incorporare nella cultura medica la scienza della complessità, in cui i risultati dipendono dalle interazioni tra i singoli elementi e non possono essere previsti da un esame dei singoli componenti. La Teoria della Complessità è stata descritta come 'l'elefante nella sala d'aspetto della Medicina, una realtà ignorata per troppo tempo'.$^{31}$ Dovremo quindi ammettere che la Medicina da qui in poi sarà tutta complessa e che la Medicina Interna non ha l'esclusiva della gestione della complessità? Questo dipenderà probabilmente da quali obiettivi si vogliono privilegiare: la malattia, il malato, la persona? Solo la terza opzione mette il medico difronte alla vera e propria complessità. Nella realtà clinica quotidiana, visto il cambiamento epidemiologico ed antropologico della società, la maggioranza dei pazienti sono complessi. La complessità, quindi, non è più un'esclusiva della Medicina Interna e/o della Geriatria: malati complessi si trovano in tutti i reparti ospedalieri sub-specialistici. La gestione della com-

\section{Quali ragioni definiscono la complessità del paziente?}

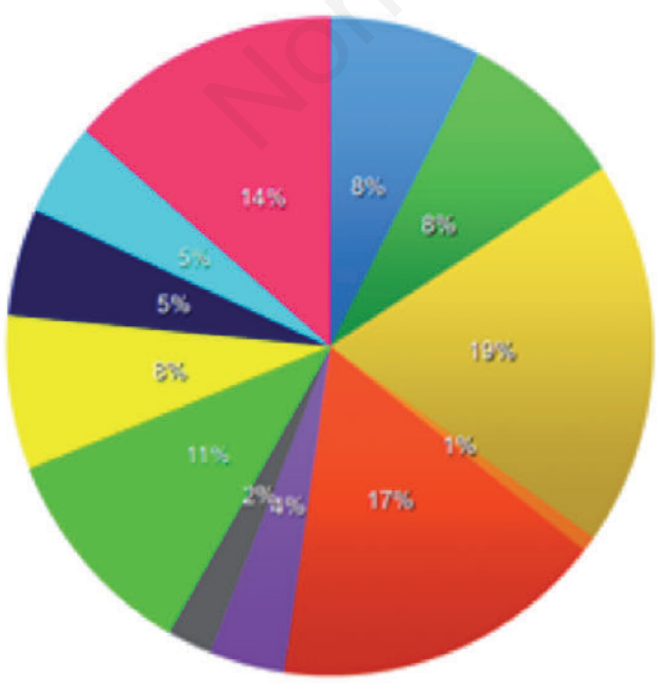

plessità continuerà però a rimanere prerogativa dell'Internista, in quanto nell'ambito delle specialità d'organo si continuerà a trattare la patologia specifica, spesso senza considerare il paziente nel suo complesso e in assenza di un effettivo coordinamento attivo dell'intero processo diagnostico-terapeutico.

\section{Gli internisti hanno ben delineato le caratteristiche salienti dei pazienti complessi}

Gli internisti hanno ben evidenziato quale complessità si rileva nel mondo reale dei reparti di Medicina Interna. Sulla base di una survey finalizzata a valutare il punto di vista degli Internisti sulla complessità è risultato che i principali elementi alla base di tale definizione stanno: i) la gravità dei pazienti/la severità dei sintomi e/o la difficoltà a migliorare, nonostante tutte le cure (failure to thrive); ii) i bisogni assistenziali: procedure mediche ed infermieristiche/gestione di devices complesse e/o avanzate in ospedale; iii) le difficoltà a definire il coordinamento delle cure e la continuità assistenziale dopo la dimissione. Seguono diverse altre problematiche, descritte nella Figura 1.32 In tema di complessità gli internisti hanno altresì considerato i concetti di clustering disease $\mathrm{s}^{33,34} \mathrm{e}$ di comorbidoma $^{35}$ con pubblicazioni ad hoc, cui si rimanda per un approfondimento.

Figura 1. Le principali cause di complessità dei pazienti ricoverati in nei reparti di Medicina Interna. 
Più recentemente FADOI, con l'introduzione degli score FCSs [(FADOI-COMPLIMED Score(s)] ha proposto gli strumenti finalizzati a misurare il livello di complessità dei pazienti ricoverati in Medicina Interna, utilizzabili per prevedere proficuamente tutti gli endpoint correlati alla complessità e, probabilmente, anche per definirne un giudizio prognostico a brevemedio termine. ${ }^{36,37}$

\section{Il ragionamento clinico non-lineare: casi semplici e complessi}

La rappresentazione del pensiero scientifico moderno trova nella 'Teoria della Complessità' di Morin la fine del razionalismo classico, lineare e semplificatore, sostituendo il concetto di 'soggetto-oggetto singolo' con quello di 'sistema', ${ }^{38}$ in un contesto globale di vari elementi in interazione fra loro. ${ }^{39}$ Tali cambiamenti concettuali introducono nella metodologia clinica strumenti improntati alla multidisciplinarietà, alla comunicazione, all'integrazione, al lavoro contestualizzato ai dati del mondo reale. ${ }^{40} \mathrm{~L}$ 'abilità di ragionamento dovrebbe essere valutata nella contingenza delle conseguenze sulle decisioni intraprese. Nel modello del pensiero lineare il percorso è generalmente ben definito: nella maggioranza dei casi si tratta di pazienti stabili, più spesso in condizioni di non urgenzaemergenza, affetti da una singola malattia, ovvero di casi semplici. Con il pensiero lineare dall'ipotesi $A$ si può giungere, anche per passaggi intermedi $1,2,3$, eccetera alla soluzione finale $B$, utilizzando le informazioni iniziali raccolte fino alla conclusione, ordinandole in modo sequenziale, come in una linea retta (Tabella 2).

Un'evidente limitazione del pensiero lineare (di per sé ripetitivo nella sua logica intrinseca) è la difficoltà a risolvere i problemi quando le cose si complicano o quando si devono affrontare problemi complessi. Nella pratica quotidiana il percorso può es- sere assai meno definito, reso complesso da una miriade di elementi interferenti, con possibili ramificazioni multiple rispetto ad un percorso netto, corrispondenti a ad altrettante alternative di scelte multiple. ${ }^{41}$ È necessario superare il concetto meccanicistico per cui i fenomeni vanno interpretati secondo dinamiche lineari proprie del pensiero riduzionistico: bisogna affrontare l'imprevedibile, richiamando le proprie capacità di pensiero creativo, rispondendo in modo flessibile a nuovi scenari complessi. ${ }^{42} \mathrm{Il}$ comportamento non lineare si verifica comunemente all'interno di sistemi contrassegnati da una natura dinamica complessa. Esso implica un possibile embricarsi di diverse situazioni nello stesso problema nello stesso paziente, per cui è necessario mettere in gioco tutte le nostre facoltà intellettive, da quelle cognitive, a quelle correlate alla memoria, all'esperienza e all'immaginazione e al 'buon senso', allo scopo di proporre risoluzioni creative. I pensatori non lineari sono in grado di sviluppare una capacità di pensiero e di problem solving estese in più direzioni nelle più difficili situazioni. Il pensiero non lineare permette alla mente di andare più in profondità alla radice del problema, in una 'spirale' rivolta verso più direzioni dalle fonti originali, per una visione complessiva e saliente dei problemi.

\section{Il caos: oltre la complessità}

L'espressione 'complessità' è di difficile comprensione, a volte anche d'impossibile spiegazione ed analisi, tanto da confondersi con il caos. ${ }^{43} \mathrm{La}$ teoria del caos descrive elementi che manifestano un comportamento sensibile alle condizioni iniziali, ma non ripetitivo e deterministico. La teoria della complessità fa un passo oltre il caos e tenta di spiegare il comportamento complesso che emerge all'interno di sistemi dinamici non lineari. ${ }^{44}$ I sistemi complessi presentano frequentemente, ma non necessariamente, un decorso caotico. Se la scienza tradizionale si occupa di feno-

Tabella 2. Esempio di un caso clinico lineare, pur se in una grande anziana.

Paziente di 94 anni, autosufficiente, con cardiopatia ipertensiva senza scompenso cardiaco, in trattamento farmacologico cronico con sartani, diuretico ed antiaggregante, senza rilevanti elementi anamnestici, salvo una pregressa frattura di femore trattata con osteosintesi e chiodo endomidollare ed una severa osteoporosi trattata con difosfonati, complicata da osteonecrosi del mascellare, trattata con successo.

Da alcuni giorni dolenzia addominale ai quadranti superiori, con Murphy dubbio e Blumberg negativo. Presenta improvvisa riacutizzazione di dolore addominale con iperpiressia fino a $39^{\circ} \mathrm{C}$.

Ricoverata per colecistite acuta e sepsi biliare in Gastroenterologia, trattata con antibiotico-terapia dopo emocoltura, terapia idratante ed elettrolitica, digiuno, viene sottoposta ad ecografia che documenta litiasi coledocica con segni ecografici di colecistite acuta ed iniziale dilatazione delle vie biliari intra-epatiche, con ittero ingravescente.

Sottoposta ad ERCP con papillo-sfinterotomia senza complicanze, viene dimessa due giorni dopo.

Il caso clinico rappresentato, riferito ad una persona molto anziana, ma autosufficiente e senza disfunzione cognitiva, dimostra che non è l'età di per sé a definire la complessità dei casi!

In questo caso la linearità era definita dall'unico problema iniziale (ittero colestatico da calcolosi con sepsi biliare), fino alla soluzione (ERCP). 
meni prevedibili, la scienza del caos concerne fenomeni non lineari ed imprevedibili, dinamici (sistemi caotici) che, pur se apparentemente incomprensibili, sottendono un ordine interno che può essere studiato con un approccio diverso da quello utilizzato nella scienza lineare. Il caos non è un disordine completo, casuale, ma al suo interno, anche se complesso, regna un ordine intrinseco. ${ }^{45-48}$ In sostanza, nei sistemi complessi si manifestano simultaneamente l'ordine ed il disordine, sia nella forma e struttura, che nel tempo. Il caos, termine facilmente utilizzato in modo equivoco, per la scienza non coincide con la casualità. Esso piuttosto corrisponde al comportamento di un sistema che sembra casuale, ma non lo è: si potrebbe definire il caos come un modo di procedere di un sistema dinamico, in funzione delle condizioni iniziali, delle variabili in causa, della loro reciproca interazione (amplificazione, attenuazione, retroazione, eccetera), con conseguente imprevedibilità dei fenomeni successivi. ${ }^{49}$ Esempi di diversi livelli di complessità clinicoassistenziale sono riportati nella Tabella $3{ }^{50}$

\section{Saggezza e buon senso}

In ogni sistema complesso economico o biologico per prendere decisioni è necessaria la saggezza, ultima tappa di un complesso processo cognitivo che va dall'attribuzione di significato ai dati all'assemblaggio delle informazioni. Tale prerogativa è utile per la comprensione dei meccanismi di utilizzo della conoscenza, che in medicina possiamo definire metodo clinico. Applicato alle organizzazioni sanitarie può essere definito come Clinical Governance. ${ }^{51}$ Il concetto pragmatico di saggezza è stato trattato da Russel Ackoff, un pioniere della scienza delle organizzazioni, nel suo scritto 'From Data to Wisdom',52 in cui per la prima volta presenta la sua gerarchia della conoscenza, adattata dagli ingegneri informatici alla scienza delle organizzazioni e al relativo decision-making. Al suo apice troviamo proprio la saggezza, ovvero la Phronesis (in greco antico $\varphi \rho o ́ v \eta \sigma \iota \varsigma)$, che, secondo Aristotele, corrisponde alla «disposizione accompagnata da ragionamento, che dirige l'agire e concerne le cose che per l'uomo sono buone e cattive», indirizzando l'azione al conseguimento dei particolari, al contrario

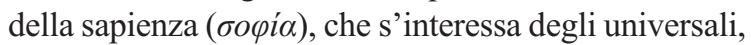
della conoscenza di quelle realtà ideali eterne che la caratterizzano come il sapere più alto che l'uomo possa raggiungere.$^{53}$ L'importanza della saggezza è stata trattata specificamente nell'ambito della clinical governance da Corrao et al. ${ }^{51}$ Essa, per evidente affinità, può essere applicata al processo decisionale clinico proprio nei casi complessi. Confucio (Dialoghi, VI-V secolo a.C.) affermava che 'Saggezza e buon senso si ottengono in tre modi: primo, con la riflessione, che è la cosa più nobile; secondo, attraverso l'imitazione, che è la cosa più semplice e, terzo, con l'esperienza, che è la cosa più amara di tutte.' Il filosofo cinese centra egregiamente il concetto riscoperto migliaia di anni dopo. La saggezza si alimenta attraverso l'esercizio della riflessione. In ambito medico, questa permette di analizzare il contesto clinico-organizzativo attraverso l'approccio cognitivo-analitico ed il riconoscimento dei pattern intuitivi, allo scopo di decidere sulle priorità, attingendo inesorabilmente dall'esperienza. Il 'buon senso' emerge quindi in maniera inconsapevole da tale complesso processo mentale, rappresentando il punto finale e più impalpabile della saggezza, riferita a risorse nascoste ma 'superiori', che attengono a capacità squisitamente individuali.

\section{Intelligenza artificiale: la logica 'fuzzy' in Medicina}

L'innovazione tecnologica condizionerà in maniera preponderante i ruoli e le funzioni del personale clinico di tutte le professioni sanitarie nel prossimo futuro. ${ }^{54}$ Nei sistemi bio-informatici intelligenti la logica fuzzy è una generalizzazione della logica standard, basata sull'attribuzione ad ogni proposizione di un grado di verità compreso nell'intervallo continuo da $0 \mathrm{a} 1 .{ }^{55}$ La logica fuzzy corrisponde alla teoria degli insiemi riferiti a categorie dai contorni indistinti. Zadeh, il matematico che introdusse questa teoria, riteneva che la logica binaria non fosse adeguata alla complessità dei problemi del mondo reale..$^{56} \mathrm{~A}$ differenza della logica binaria, la logica fuzzy tende ad eliminare l'incertezza, incorporando la vaghezza che contrassegna i sistemi complessi. Con essa si realizza un modo di 'ragionamento approssimato con concetti definiti in modo vago' ${ }^{57}$ La logica 'fuzzy', ovvero 'sfocata', 'sfumata', 'indistinta', 'confusa', applicata alla pratica clinica significa ridimensionamento delle certezze e delle verità assiomatiche, passando da una medicina delle certezze a quella delle probabilità, ${ }^{58,59}$ ovvero dalla verità inoppugnabile alla possibilità della 'vaghezza' del mondo reale. ${ }^{60}$ La mancanza di informazioni, le possibili imprecisioni e, spesso, la natura contraddittoria dei dati disponibili sono comuni nella pratica medica. ${ }^{61}$ Nella logica fuzzy (definita anche 'logica vaga' o, addirittura, 'logica illogica') il metodo di ragionamento 'logico' tende a giungere a decisioni corrette anche quando i dati a disposizione sono approssimativi, imprecisi o incerti. Essa tende a rappresentare la realtà mediante valori diversi dai semplici 'vero-falso', 'bianco-nero' o '0-1', insite nella logica classica: essa valuta la gamma dei valori compresi tra il 'completamente vero' e il 'completamente falso', utilizzando valori compresi tra 0 e 1 (inclusi) e tutte le sfumature di un mondo fatto principalmente di grigi, di verità e di elementi parziali, anche apparentemente scollegati tra loro. ${ }^{62}$ La logica fuzzy riveste un ruolo importante in 
medicina. ${ }^{63-67} \mathrm{Un}$ approccio logico tradizionale va sicuramente usato quando il dominio è limitato e ben definito, quando interessa solo la falsità o la verità di un fatto, quando si ritiene non importante rappresen- tare l'incertezza. Un sistema che presenta incertezze, invece, potrebbe essere gestito con l'utilizzo del calcolo della probabilità che descrive la possibilità che un evento sia Vero (o Falso), legata comunque alla lo-

Tabella 3. Livelli di complessità clinico-assistenziale: implicazioni e modelli di riferimento.

\begin{tabular}{|c|c|c|c|c|c|c|}
\hline \multirow[t]{2}{*}{ Livello } & \multirow[t]{2}{*}{ Il contesto è: } & \multirow[t]{2}{*}{ Esempio: } & \multicolumn{2}{|c|}{ Caratteristiche } & \multirow{2}{*}{$\begin{array}{l}\text { Azioni richieste - } \\
\text { vi è bisogno di: }\end{array}$} & \multirow{2}{*}{$\begin{array}{l}\text { Modello principale } \\
\text { di riferimento ai fini } \\
\text { decisionali }\end{array}$} \\
\hline & & & $\begin{array}{l}\text { Intensità } \\
\text { assistenziale } \\
\text { richiesta }\end{array}$ & $\begin{array}{l}\text { Trattamento } \\
\text { richiesto }\end{array}$ & & \\
\hline $\mathbf{0}$ & $\begin{array}{l}\text { Conosciuto, per } \\
\text { nulla complesso, } \\
\text { spesso } \\
\text { contrassegnato } \\
\text { da una singola } \\
\text { malattia }\end{array}$ & $\begin{array}{l}\text { CAP batterica in } \\
\text { paziente giovane } \\
\text { senza comorbidità }\end{array}$ & $\begin{array}{l}\text { Paziente stabile } \\
\text { senza apparenti } \\
\text { co-morbidità }\end{array}$ & $\begin{array}{l}\text { Il farmaco o } \\
\text { l'associazione di } \\
\text { farmaci più indicata } \\
\text { secondo le evidenze } \\
\text { scientifiche }\end{array}$ & $\begin{array}{l}\text { Cure standard } \\
\text { di routine } \\
\text { necessarie di } \\
\text { qualità }\end{array}$ & $\begin{array}{l}\text { EBM } \\
\text { Linee guida } \\
\text { Giudizio clinico } \\
\text { Opinioni del paziente }\end{array}$ \\
\hline 1 & $\begin{array}{l}\text { Leggermente } \\
\text { complesso, } \\
\text { ma prevedibile }\end{array}$ & $\begin{array}{l}\text { CAP in paziente con } \\
\text { insufficienza renale } \\
\text { e peggioramento } \\
\text { della funzione } \\
\text { respiratoria }\end{array}$ & $\begin{array}{l}\text { Paziente stabile, } \\
\text { ma a rischio } \\
\text { d'instabilità }\end{array}$ & $\begin{array}{l}\text { Il farmaco o } \\
\text { l'associazione di } \\
\text { farmaci più } \\
\text { indicati aggiustati } \\
\text { per la funzione } \\
\text { renale e } \\
\text { considerando } \\
\text { l'interazione } \\
\text { con i farmaci che } \\
\text { il paziente assume } \\
\text { routinariamente }\end{array}$ & $\begin{array}{l}\text { Pianificazione } \\
\text { dell'assistenza di } \\
\text { base mirata ai } \\
\text { bisogni attuali con } \\
\text { monitoraggio } \\
\text { dei parametri vitali e } \\
\text { alla prevenzione } \\
\text { secondaria }\end{array}$ & $\begin{array}{l}\text { PDTA } \\
\text { Linee guida } \\
\text { EBM } \\
\text { Giudizio clinico } \\
\text { Opinioni del paziente }\end{array}$ \\
\hline 2 & $\begin{array}{l}\text { Complesso, } \\
\text { non prevedibile } \\
\text { nella sua } \\
\text { evoluzione }\end{array}$ & $\begin{array}{l}\text { CAP in anziano } \\
\text { ospedalizzato } \\
\text { complicato da } \\
\text { trombosi venosa } \\
\text { profonda (TVP) } \\
\text { ed embolia } \\
\text { polmonare }\end{array}$ & $\begin{array}{l}\text { Paziente } \\
\text { potenzialmente } \\
\text { instabile, a rischio } \\
\text { di complicanze } \\
\text { anche gravi }\end{array}$ & $\begin{array}{l}\text { Protocollo per } \\
\text { trattamento } \\
\text { dell'embolia } \\
\text { polmonare in base } \\
\text { alla gravità }\end{array}$ & $\begin{array}{l}\text { Assistenza } \\
\text { sub-intensiva/ } \\
\text { intensiva, } \\
\text { pianificazione } \\
\text { dell'assistenza } \\
\text { di base mirata } \\
\text { ai bisogni }\end{array}$ & $\begin{array}{l}\text { Giudizio clinico } \\
\text { Valutazione delle } \\
\text { priorità EBM } \\
\text { (Linee guida) } \\
\text { (PDTA) } \\
\text { Opinioni del } \\
\text { paziente }\end{array}$ \\
\hline 3 & $\begin{array}{l}\text { Molto complesso } \\
\text { per gravi } \\
\text { comorbilità e } \\
\text { difficoltà gestionali }\end{array}$ & $\begin{array}{l}\text { CAP in paziente } \\
\text { affetta da } \\
\text { insufficienza renale } \\
\text { con sindrome da } \\
\text { anticorpi } \\
\text { anti-fosfolipidi, } \\
\text { allettata per grave } \\
\text { emorragia intestinale } \\
\text { da angiodisplasia } \\
\text { del tenue con recente } \\
\text { riscontro di TVP } \\
\text { bilaterale agli arti } \\
\text { inferiori }\end{array}$ & $\begin{array}{l}\text { Paziente instabile, } \\
\text { a rischio di } \\
\text { complicanze }\end{array}$ & $\begin{array}{l}\text { Gestione mirata } \\
\text { delle singole } \\
\text { patologie in accordo } \\
\text { con le interazioni } \\
\text { fra la diverse } \\
\text { comorbidità; } \\
\text { valutazione delle } \\
\text { diverse linee guida } \\
\text { contestualizzate } \\
\text { al singolo caso }\end{array}$ & $\begin{array}{l}\text { Assistenza intensiva } \\
\text { pianificata sui } \\
\text { bisogni }\end{array}$ & $\begin{array}{l}\text { Valutazione delle } \\
\text { priorità } \\
\text { Giudizio clinico } \\
\text { (EBM) } \\
\text { (Linee guida) } \\
\text { Opinioni del paziente }\end{array}$ \\
\hline 4 & $\begin{array}{l}\text { Caotico: troppi } \\
\text { elementi da gestire }\end{array}$ & $\begin{array}{l}\text { Paziente con } \\
\text { polmonite } \\
\text { nosocomiale, affetto } \\
\text { da demenza senile, } \\
\text { con sondino } \\
\text { naso-gastrico e } \\
\text { ulcere da pressione, } \\
\text { diabete mellito } \\
\text { complicato da } \\
\text { insufficienza renale, } \\
\text { con valvola mitralica } \\
\text { meccanica in TAO, } \\
\text { recente trauma cranico } \\
\text { con ematoma } \\
\text { intraparenchimale } \\
\text { inoperabile. Vive solo } \\
\text { in precarie condizioni } \\
\text { economiche }\end{array}$ & $\begin{array}{l}\text { Paziente stabile/ } \\
\text { instabile con } \\
\text { co-morbilità e } \\
\text { situazioni di } \\
\text { contesto } \\
\text { non facilmente } \\
\text { risolvibili } \\
\text { o }\end{array}$ & $\begin{array}{l}\text { Terapia finalizzata } \\
\text { al miglioramento } \\
\text { della qualità di vita, } \\
\text { in base alla } \\
\text { selezione dei } \\
\text { bisogni soggettivi } \\
\text { ed oggettivi }\end{array}$ & $\begin{array}{l}\text { Assistenza } \\
\text { pianificata } \\
\text { sulla selezione } \\
\text { delle priorità e dei } \\
\text { bisogni } \\
\text { primari }\end{array}$ & $\begin{array}{l}\text { Buon senso e } \\
\text { implicazioni etiche } \\
\text { Selezione delle } \\
\text { priorità } \\
\text { Giudizio clinico } \\
\text { (EBM) } \\
\text { Opinioni del paziente }\end{array}$ \\
\hline
\end{tabular}

CAP, polmonite acquisita comunitaria; TVP, trombosi venosa profonda; EBM, evidence based medicine; PDTA, percorsi diagnostico-terapeutici assistenziali. Il modello principale di riferimento ai fini decisionali indica i vari elementi disponibile per le decisioni in ordine decrescente di prevalente utilizzo; le parentesi indicano la probabile indisponibilità di informazioni del relativo elemento decisionale. Modificata $\mathrm{da}^{16,50}$. 
gica classica. ${ }^{68}$ L'approccio fuzzy supera ciò e restituisce un valore (come nella probabilità) tra 0 e 1 che rappresenta quanto una proposizione sia vera. Lo sviluppo di relazioni fuzzy in medicina e la loro applicazione nella diagnosi assistita potrebbe costituire un ulteriore metodo di approccio per affrontare casi complessi in assenza di confini netti dell'insieme di sintomi, le diagnosi possibili e modalità di presentazione delle malattie. ${ }^{69}$ In conclusione, la logica fuzzy è utile quando disponiamo di molte informazioni, in presenza di dati quali/quantitativi scalari. Sempre più lo sviluppo della tecnologia potrà offrire nuovi strumenti per misurare $\mathrm{i}$ parametri patologici complessi ed è ragionevole pensare che essa sarà impiegata sempre di più nella diagnosi clinica. ${ }^{70}$ Stando attenti, però, ai possibili svantaggi della logica fuzzy e ad evitare di subire una 'medicina del pallottoliere', con la quale rischiamo di rinunciare ad un approccio idiografico della valutazione clinica, che è sempre multi-dimensionale, dovendo considerare aspetti variegati (psicologici, etici, sociali, assistenziali, religiosi, oltre che fisiopatologici e terapeutici personalizzati), ${ }^{71}$ ovvero tener conto del contesto complessivo, cosa che, allo stato attuale, nessuna intelligenza artificiale è in grado di fare.

\section{Complessità, incertezza ed inerzia clinica nelle decisioni mediche}

La complessità accentua il rischio di errore e facilita l'inerzia clinica decisionale. ${ }^{72} \mathrm{La}$ medicina è un'arte stocastica, aleatoria e casuale, che impone spesso, nell'assunzione delle decisioni, di tener conto di variabili probabilistiche non deterministiche (deterministico significa che non vi è incertezza: da certe premesse si generano sempre le stesse conseguenze), con un ampio margine di casualità imprevedibile e di conseguente incertezza. Per effettuare una prognosi nei pazienti in Medicina Interna, particolarmente se in fase avanzata di malattia, non dobbiamo 'guardare nella sfera di cristallo', ma dovremmo poter disporre di dati oggettivi, su studi ad hoc. ${ }^{73,74}$ L'incertezza inerente alla cura del paziente resta un elemento determinante nella pratica clinica, nonostante i progressi della medicina basata sulle evidenza. ${ }^{75,76}$ Essa può essere ridotta con i progressi metodologici ed informatici, ma mai completamente eliminata dal processo decisionale. La maggior parte delle decisioni assunte in medicina contiene elementi intuitivi, facilmente criticabili quando sono basati su pregiudizi e distorsioni cognitive. ${ }^{77,78}$ L'incertezza è onnipresente in medicina, ma spesso viene sottovalutata. Al contrario l'ammissione dell'incertezza può costituire il punto di partenza per facilitare la sinergia tra paziente e medico. ${ }^{79}$ Essa dovrebbe essere accolta dai medici quale straordinaria opportunità nell'impegnarsi in una relazione di cura empatica, su livelli più profondi, ${ }^{80}$ in una dimensione olistica, meno probabilistica e più umana, per fornire cure più appropriate e personalizzate. ${ }^{81} \mathrm{In}$ una logica di attenuazione delle conseguenze correlate all'incertezza sarebbe necessario riconoscere i limiti oggettivi esistenti: ${ }^{82}$ i) il dominio incompleto e imperfetto del sapere dal medico, che non può possedere tutte le conoscenze del sapere; ii) i limiti propri della conoscenza medica attuale su diverse problematiche cui nessun professionista, pur molto esperto, può dare risposte sempre adeguate; iii) l'oggettiva difficoltà di distinguere l'ignoranza o l'incapacità personale dai limiti intrinseci della conoscenza medica. ${ }^{83}$ Creare una cultura che riconosca l'incertezza medica come fattore imprescindibile nel decision making potrebbe essere un primo passo per insegnare ai medici una maggiore tolleranza ed adattabilità nei suoi confronti. ${ }^{84}$

\section{Conclusioni}

La competenza fondata sulla conoscenza è essenziale per comprendere la complessità. Come internisti non possiamo affrontare il mondo reale in termini assoluti, come 'bianco' o 'nero', abdicando alla sfida di dover tentar di gestire la complessità. In Medicina Interna il mondo reale non è quello delle certezze. Esso è costituito frequentemente da problemi confusi, sfocati, contestuali a variegate situazioni bio-psico-sociali e cliniche. La conoscenza di tali fattori dovrebbe far parte della cultura del medico internista, come componente fondamentale dell'apprendimento e della competenza, intesa come capacità d'individuare e risolvere i problemi, mediante una valutazione multidimensionale e creativa della situazione nel suo insieme, dando priorità ai problemi salienti ${ }^{85}$ tenendo conto del contesto globale, cosa che, allo stato, nessuna intelligenza artificiale è in grado di fare. Sono necessarie nuove prospettive concettuali in grado di garantire visione dinamica, sistemica ed intuitiva del mondo, superando i tradizionali approcci fondati sul 'ridurre e risolvere' la complessità dei casi sia nell'assistenza clinica che nell'organizzazione dei servizi. ${ }^{86}$ In tale ambito vediamo ancora poche proposizioni efficaci di ricerca clinico-gestionale da implementare nel prossimo futuro per il nostro Servizio Sanitario pubblico. Chi ha responsabilità politiche e manageriali deve capire l'economia di scala e il valore aggiunto potenziale della Medicina Interna per l'organizzazione e la gestione clinico-diagnostico-terapeutica ottenibile delegando ai sub-specialisti il top e le competenze distintive della loro disciplina (pochi ma buoni) e agli internisti (molti ma buoni) il coordinamento delle attività cliniche, non solo dei reparti internistici, ma anche di quelli chirurgici, gravati da pazienti complessi con molteplici problematiche mediche, secondo la visione italiana del modello dell' $h o-$ spitalist. ${ }^{87}$ 


\section{Post scriptum}

Nel manoscritto abbiamo voluto mantenere diversi termini inglesi originali 'virgolettati' o in corsivo non per una auspicata anglofilia o per ripudio della lingua italiana. La lingua inglese domina la letteratura scientifica medica di qualità. Abbiamo voluto facilitare la lettura delle principali parole chiave sul tema e dei molti articoli di riferimento, con un glossario pertinente all'argomento affrontato.

\section{Bibliografia}

1. Scandellari C. Medicina degli schiavi e dei liberi, Tutor 2014, 3: 6-11.

2. Bocchi G, Ceruti M. La sfida della complessità a cura di, Bruno Mondadori, 2007.

3. Turne BJ, Cuttle L. The Complexity of Measuring Clinical Complexity, Ann Intern Med. 2011; 155: 851-852.

4. Ministero della Salute. Criteri di Appropriatezza clinica, tecnologica e strutturale nell'assistenza al paziente complesso. Quaderni del Ministero della Salute 2013.

5. Greco P. Alla ricerca della complessità, https://www. scienzainrete.it/contenuto/articolo/pietro-greco/alla-ricerca-della- complessita/aprile-2013

6. Weaver W. Science and complexity, American Scientist 1948, 36: 536.

7. Macenni C. Applicazioni del in Medicina Interna Caos e complessità, Istituto sui Sistemi Complessi-Prof. L. Pietronero, http://www.isc.cnr.it/ita/aboutisc/index.html

8. Macenni C. Applicazioni del in Medicina Interna Caos e complessità, Istituto sui Sistemi Complessi-Prof. L. Pietronero, http://www.isc.cnr.it/ita/aboutisc/index.html

9. Polenta S. La psicologia della Gestalt come teoria della complessità, L'analisi qualitativa dell'esperienza diretta ISBN 978-88-548-2916-9 DOI 10.4399/ 978885482916917 pp. 185-193 (novembre 2009).

10. Macenni C. Applicazioni del in Medicina Interna Caos e complessità, Istituto sui Sistemi Complessi-Prof. L. Pietronero, http://www.isc.cnr.it/ita/aboutisc/index.html

11. Bellavite P. La Complessità in Medicina. Fondamenti di un approccio sistemico-dinamico alla salute, alla patologia e alle terapie integrate, Tecniche Nuove Ed, Milano, 2009.

12. Lewin K. Teoria dinamica della personalità (1935), tr. it., Editrice Universitaria, Firenze 1965.

13. Nardi R, Fabbri T, Belmonte G et al. Medicina interna, paziente complesso, evidence based medicine e le non evidenze, Italian Journal of Medicine 2009, 3, 191-200.

14. Whittle J, Bosworth H. Studying complexity is complex. J Gen Intern Med 2007;22 Suppl 3:379-81.

15. Facchinetti G. Problemi complessi, nuovi strumenti, https://www.unisalento.it/documents/20152/299800/lezione+spec+14-11-17.ppt/8045daec-ff55-99e8-3bc5$9 \mathrm{~d} 2 \mathrm{e} 2 \mathrm{fb} 59 \mathrm{~b} 7 \mathrm{~d}$ ?version=1.0\&downloa $\mathrm{d}=$ true

16. Agency for Healthcare Research and Quality (AHRQ). Definition: Complex Patient, Funding Opportunity Announcement (FOA). Technical Assistance Conference Call. October 15, 2007.

17. Safford MM, Allison JJ, Kiefe CI. Patient complexity: more than comorbidity. The vector model of complexity.
J Gen Intern Med 2007;22 Suppl 3:382-390.

18. Upshur RE, Tracy S. Chronicity and complexity: is what's good for the diseases always good for the patients? Can Fam Physician 2008;54(12):1655-658.

19. Nardi R, Scanelli G, Corrao S, Iori I, Mathieu G, Cataldi Amatrian R. Co-morbidity does not reflect complexity in internal medicine patients. Eur J Intern Med 2007;18/ 5: 359-368.

20. Gensini GF, Conti AA. Il quesito clinico, RIMeL/IJLaM 2005; 1 (Suppl), pp: 24-27.

21. Singh H, Petersen LA, Thomas EJ. Understanding diagnostic errors in medicine: a lesson from aviation. Qual Saf Health Care 2006; 15/3: 159-164.

22. Glass L, Mackey MC. From clocks to chaos: the rhythms of life, NJ, Princeton University Press, 1988.

23. Borghi C, Desideri GB, Ferspil C. Percorso formativo in Esperto in Medicina della complessità, https://www. sys-academy.org/esperto-medicina-della-complessita/

24. Senin U, Cherubini A, Necocco P. Paziente Anziano Paziente Geriatrico Medicina della Complessità, EdiSES, III / 2010.

25. Miles A. Complexity in medicine and healthcare: people and systems, theory and practiceje, Journal of Evaluation in Clinical Practice 2009, 15: 1356-1294.

26. Uberti M. La complessità in medicina, Rivista della Società Italiana di Medicina Generale 2009, 1: 19-22.

27. Islam R, Weir C, Del Fiol G. Clinical Complexity in Medicine: A Measurement Model of Task and Patient Complexity, Methods Inf Med 2015; 55/1: 14-22.

28. Manca M. Handbook of Complexity in Medicine, Springer Verlag; 1st ed. 2025 edizione-14 febbraio 2016.

29. Kuipers P, Kendall E, Ehrlich C, et al. Complexity and health care: Health practitioner workforce, services, roles, skills and training to respond to patients with complex needs. Brisbane: Clinical Education and Training Queensland, 2011, https://www.health.qld.gov. au/_data/assets/pdf_file/0027/150768/complexcarefull1.pdf

30. Tonelli M, Wiebe N, Manns BJ et al. Comparison of the Complexity of Patients Seen by Different Medical Subspecialists in a Universal Health Care System, JAMA Netw Open 2018, 1/7: e184852. doi:10.1001/jamanetworkopen.2018.4852

31. Alexander CJ. Complexity \& Medicine: The Elephant in the Waiting Room, Nottingham University Press; 1 edition (27 Sept. 2010).

32. Gambacorta M, Montagnani A, Gnerre P. Risultati di una survey sulla complessità promossa da FADOI, in: Panigada G, Campanini M, Fontanella A, Nardi R, Aspetti clinico-organizzativi nella degenza medica ospedaliera in Italia: il ruolo della Medicina Interna nel Dipartimento Medico e continuità assistenziale, Quaderni Italian Journal of Medicine, 2015, 3/1: 499-612.

33. Marengoni A, Bonometti F, Nobili A, et al. In-hospital death and adverse clinical events in elderly patients according to disease clustering: the REPOSI study. Rejuven Res 2010;13:469-77.

34. Tangianu F, Gnerre P, Colombo F et al. Could clustering of comorbidities be useful for better defining the internal medicine patients' complexity? Italian Journal of Medicine 2018, 12: 137-144.

35. Corrao S. 2018-Data from the Reposi Registry.

36. Bonizzoni E, Gussoni G, Agnelli G, Antonelli Incalzi R, 
Bonfanti M, Mastroianni F, et al. The complexity of patients hospitalized in Internal Medicine wards evaluated by FADOI-COMPLIMED score(s). A hypothetical approach, PLoS ONE 2018, 13(4): e0195805.

37. Nardi R, Nozzoli C, Berti F, Bonizzoni E, Fabbri LM, Frasson $\mathrm{S}$ et al. Prognostic value for mortality of the new FADOI-COMPLIMED score(s) in patients hospitalized in medical wards, PLoS ONE 2019, 14/7: e0219767. https://doi.org/10.1371/journal.pone.0219767).

38. Morin E. I sette saperi necessari all'educazione del futuro, Raffaello Cortina Editore, 2000.

39. Fondazione Paolo Procacci, Medicina della Complessità: la moderna Medicina Interna, http://www.fondazioneprocacci.org/OLD/e107_files/downloads/med_comp lessita.pdf

40. Buckman R. La comunicazione della diagnosi, Raffaello Cortina Editore, 1992.

41. Whelan A. Reforming case-based learning with non-linear gameplay: the potential of branched narratives and virtual patient models, UOJM 2014, 4/1: 24-27.

42. Cacciatore F. La VMD nella gestione clinica della complessità, $57^{\circ}$ Congresso Nazionale SIGG, 21-24 novembre 2012.

43. Benkirane R. La teoria della complessità, Bollati Boringhieri, 2007.

44. Higgins JP. Nonlinear systems in medicine, Yale J Biol Med. 2002; 75/5-6: 247-260.

45. Codolini G. Teorie del caos, https://codolini.wordpress. com/approfondimenti-di-varie-discipline/approfondimenti-di-fisica/teorie-del-caos/

46. Grossi E. Teoria del caos: influenze positive sulla medicina pratica, http://www.geragogia.net/editoriali/ caos.html

47. Grossi E. La sfida della complessità nelle malattie croniche degenerative: ruolo attuale dei sistemi artificiali adattivi, http://www.arisla.org/wp-content/uploads/ 2013/03/Seminario-22-Marzo-2013

48. Kumar A, Hegde BM. Chaos theory: impact on and applications in medicine, Nitte University Journal of Health Science 2012, 2/4: 93-99.

49. Bellavite $\mathrm{P}$, Zatti M. Il paradigma della complessità nelle scienze e in medicina, Informasalus.it, 23 marzo 1996.

50. Baird M, Peek CJ, Gunn W, Valeras A. Approaches to complexity care. Chapter in The Landscape of Collaborative Healthcare: Evaluating the Evidence, Identifying the Essentials, M. Talen \& A.B. Valeras (Eds.), Springer Science \& Business Media, 2013.

51. Corrao S, Arcoraci V, Arnone S, Calvo L, Scaglione R, Di Bernardo C, Lagalla R, Caputi AP, Licata G. Evidence-Based Knowledge Management: an approach to effectively promote good health-care decision-making in the Information Era, Intern Emerg Med. 2009, 4/2: 99-106.

52. Ackof RL, From Data to Wisdom 1999, Ackoff's Best. New York: John Wiley \& Sons: 170-172.

53. Aristotele, Etica Nicomachea, VI, 5, 1140 b 4.

54. Topol E. Deep Medicine: How Artificial Intelligence Can Make Healthcare Human Again, Basic Books; 1 edition (March 12, 2019).

55. Grossi E. Medical concepts related to individual risk are better explained with 'plausibility' rather than 'probability', BMC Cardiovascular Disorders 2005, 5:31 doi:10.1186/1471-2261-5-31.
56. Zadeh LA. The Role of Fuzzy Logic in the Management of Uncertainty in Expert Systems, Fuzzy Sets and Systems 1983, 11: 199-227.

57. Simani S. Introduzione alla Logica Fuzzy http://www. silviosimani.it/Logica_Fuzzy_TdS_2x.pdf

58. Cagli V. La crisi della diagnosi. Cosa è mutato nel concetto e nelle procedure della diagnosi medica, Armando Ed, Roma 2007.

59. Cagli V. Come si ragiona in Medicina, il dialogo tra sapere, esperienza, logica e intuizione, Armando Ed, Roma 2013.

60. Pareti G. Dalla parresia alla logica fuzzy. La difficile pratica del 'discorso vero' in medicina, https://iris.unito.it/ retrieve/handle/2318/1677520/440823/fuzzy.pdf

61. Abbod MF, von Keyserlingk DG, Linkens DA, Mahfouf M. Survey of utilisation of fuzzy technology in Medicine and Healthcarem, Fuzzy Sets and Systems 2001, 120/2: 331-349.

62. Facchinetti G, Franci F, Marchese L et al. La cultura dell'incertezza - Fuzzy, Logica, Realtà, Sanità, Arte, Eurilink Campus, University Press, 2016.

63. Abbod MF, von Keyserlingk DG, Linkens DA, Mahfouf M. Survey of utilisation of fuzzy technology in Medicine and Healthcare. Fuzzy Sets and Systems. 2001; 120/2: 331-349.

64. Boegl K, Adlassnig KP, Hayashi Y, Rothenfluh TE, Leitich $\mathrm{H}$. Knowledge acquisition in the fuzzy knowledge representation framework of a medical consultation system. Artificial Intelligence in Medicine. 2004, 30/1: 1-26.

65. Mahfouf M, Abbod MF, Linkens DA. A survey of fuzzy logic monitoring and control utilization in medicinel, Artificial Intelligence in Medicine 2001, 21/1-3: 27-42.

66. Steimann F. On the use and usefulness of fuzzy sets in medical AI, Artificial Intelligence in Medicine 2001; 21/1-3: 131-137.

67. Szczepaniak PS, Lisoba PJG, Kacprzyk J, Fuzzy Systems in Medicine. Heidelberg, Germany: Physica; 2000.

68. De Simone M. La tecnologia fuzzy- teoria e pratica nei sistemi esperti, Tesina di intelligenza artificiale, https://areeweb.polito.it/didattica/gcia/Materiale_Didattico/Approfondimenti/FuzzyLogic/fuzzylogic.pdf

69. Seising R. From vagueness in medical thought to the foundations of fuzzy reasoning in medical diagnosis, Artif Intell Med 2006, 38/3: 237-256.

70. Licata G. Probabilistic and fuzzy logic in clinical diagnosis, Int Emergency Med 2007, 2: 100. https://doi.org/10.1007/s11739-007-0051-9

71. Prattichizzo F, La medicina del pallottoliere, Toscana Medica, 15 giugno 2018, http://www.toscanamedica. org/87-toscana-medica/qualita-e-professione/674-lamedicina-del-pallottoliere

72. Miles RW. Management Reasoning Beyond the Diagnosis, J Am Med Dir Assoc 2007, 8/6: 349-354; CANMeds, Medical expert, key concepts, Complexity, uncertainty, and ambiguity in clinical decision-making, http://www.royalcollege.ca/rcsite/canmeds/framework/c anmeds-role-medical-expert-e

73. Sonnenberg FA, Beck JR. Markov models in medical decision making: a practical guide, Med Decis Making, 1993, 13/4: 322-338.

74. Nardi R, Belmonte G, Lusiani L et al. Appendice I Metodi di riconoscimento e di valutazione del paziente in 
fase terminale o a rischio di elevata mortalità in ospedale, Quad It J Med, 2017, 5/4: 111-145.

75. Tanenbaum SJ. Commentary: Uncertainty, consultation, and the context of medical Care, BMJ 2005, 330: 515.

76. Griffiths F, Green E, Tsouroufli M. The Nature Of Medical E-ùvidence And Its Inherent Uncertainty For The Clinical Consultation: Qualitative Study, BMJ 2005, 330/7490: 511-515.

77. Hall KH. Reviewing intuitive decision-making and uncertainty: the implications for medical education, Med Educ 2002 36/3: 216-224.

78. Bhise V, Rajan SS, Sittig DF, Morgan RO et al. Defining and Measuring Diagnostic Uncertainty in Medicine: A Systematic Review, J Gen Intern Med. 2018; 33/1: 103115.

79. Hatch S. Uncertainty in medicine, Editorials, BMJ 2017; 357: j2180.

80 . Wellbery C. The valur of medical uncertainly? The Lancet 2010, 375 (9727):n1686-1687.
81. Editorial, Uncertainly in medicine, The Lancet 2010, 375/9727: 1666.

82. Fox RC. The Evolution of Medical Uncertainty, Milbank Memorial Fund Quarterly/Health and Society 1980, 58/1: 1-49.

83. Spinsanti S. Certezze e incertezze del sapere medico, in: Bioetica e antropologia medica, La Nuova Italia Scientifica, Roma 1991, pp. 71-81.

84. Kim K, Lee YM. Understanding uncertainty in medicine: concepts and implications in medical education, Korean J Med Educ 2018; 30/3: 181-188.

85. Fraser SW, Greenhalgh T. Coping with complexity: educating for capability, BMJ 2001, 323/7316: 799-803

86. Plsek PE, Greenhalgh T. Complexity science: The challenge of complexity in health care, BMJ 2001; 323/7313: 625-628).

87. Orlandini F, La Regina M, Fontanella A, Campanini M, Gnerre P. La figura dell'Hospitalist in Italia, Italian Journal of Medicine, 2018, 6/1: 1-57. 


\section{Competenza e metodologia in Medicina Interna: quali strumenti di valutazione delle capacità di ragionamento clinico? Prima Parte}

Flavio Tangianu, ${ }^{1}$ Antonino Mazzone, ${ }^{2}$ Giuliano Pinna, ${ }^{3}$ Giuseppe Chesi, ${ }^{4}$ Paola Gnerre, ${ }^{5}$ Franco Berti,${ }^{6}$ Michele Stornello, ${ }^{7}$ Carlo Nozzoli,${ }^{8}$ Salvatore Corrao, ${ }^{9}$ Mauro Silingardi, ${ }^{10}$ Giovanni Mathieu, ${ }^{11}$ Andrea Fontanella, ${ }^{12}$ Daniele Borioni, ${ }^{13}$ Roberto Nardi ${ }^{13}$

${ }^{1}$ Medicina Interna, Dipartimento di Medicina, Università di Insubria (VA); ${ }^{2}$ Medicina Interna, Legnano (MI); ${ }^{3}$ Medicina Interna, Asti; ${ }^{4}$ Dipartimento di Medicina Interna, Ospedale Magati di Scandiano, AUSL di Reggio Emilia; ${ }^{5}$ Medicina Interna 2, Ospedale San Paolo, Savona; ${ }^{6}$ Medicina Interna, Roma; ${ }^{7}$ Medicina Interna, Siracusa; ${ }^{8}$ Medicina Interna, Azienda Ospedaliero-Universitaria Careggi, Firenze; ${ }^{9}$ Dipartimento di Medicina Clinica, ARNAS Civico Di Cristina Benfratelli, Palermo; PROMISE, Università di Palermo; ${ }^{10}$ Medicina Interna, Ospedale Maggiore, Bologna; ${ }^{11}$ Medicina Interna, ASL Torino 3, Piemonte; ${ }^{12}$ Medicina Interna, Ospedale Madonna del Buon Consiglio Fatebenefratelli, Napoli; ${ }^{13}$ Medicina Interna, Bologna, Italia

\section{Introduzione}

Il ragionamento clinico è una componente essenziale della pratica medica, ma non esistono definizioni univoche di tale concetto. Esso si compendia in tutti i procedimenti logici coinvolti nel percorso diagnostico in cui, si integrano tutte le conoscenze acquisite, la capacità di valutazione delle evidenze disponibili e dei valori in gioco. Di fatto l'internista applica, anche in maniera implicita e contemporanea, diversi modelli di ragionamento clinico, corrispondenti ad abilità e competenze variegate.

\section{Prima Parte: Il Ragionamento Clinico}

\section{Il ragionamento clinico}

Il ragionamento clinico è una componente essenziale della pratica medica, ma non esistono definizioni univoche di tale concetto. Sono state proposte diverse denominazioni alternative, impiegate in maniera intercambiabile, quali 'giudizio clinico' (clinical judgment), 'pensiero clinico','pensiero critico', 'processo decisionale', 'capacità di risoluzione

Corrispondente: Roberto Nardi, Medicina Interna, Bologna, Italia.

E-mail: nardidoc48@gmail.com

Articolo pubblicato secondo la Creative Commons Attribution NonCommercial 4.0 License (CC BY-NC 4.0).

${ }^{\circ}$ Copyright: the Author(s), 2020

Licensee PAGEPress, Italy

QUADERNI - Italian Journal of Medicine 2020; 8(1):91-104 dei problemi o problem solving'. 'Pensiero critico' e 'ragionamento clinico' sono termini impiegati spesso in maniera indifferente; in realtà pensiero critico è un termine più ampio che include il ragionamento clinico. Il 'ragionamento clinico' è un termine specifico, che indica le modalità di valutazione e gestione dei problemi del paziente. Molto più semplicemente potrebbe essere definito come esercizio del pensiero finalizzato ad una decisione ragionevole sulla prevenzione, diagnosi e/o trattamento di un specifico problema clinico. ${ }^{1}$ Tale processo permette di elaborare le conoscenze e le informazioni per la selezione dei migliori interventi diagnostici e/o terapeutici e/o preventivi. Nella progressione del ragionamento clinico, entrano in gioco vari tipi di conoscenza (esplicita e tacita), il modello duale di pensiero (intuitivo e analitico), l'esperienza, il ragionamento logico-sequenziale ed ipotetico deduttivo ed il ragionamento bayesiano. In sintesi il ragionamento clinico si compendia in tutti i procedimenti logici coinvolti nel percorso diagnostico, in una complessa interazione fra conoscenza, esperienza e contesto operativo del singolo professionista, in grado di condizionare le modalità di progressione di tale processo, dall'acquisizione dei dati alla decisione finale. ${ }^{2,3}$ Il confronto tra le informazioni disponibili (dati epidemiologici, anamnestici ed obiettivi, laboratoristico-strumentali) e il repertorio di conoscenze (schemi nosografici, expertise professionale, competenza) porta all'elaborazione delle ipotesi diagnostiche, selezionandone alcune ed abbandonandone di volta in volta altre, meno probabili. $\mathrm{Nel}$ ragionamento clinico, in sostanza, si integrano tutte le conoscenze acquisite, la capacità di valutazione delle evidenze e dei valori in gioco, attingendo all'irrinunciabile esperienza maturata e alle capacità comunicative, per l'assunzione di decisioni condivise 
con il paziente per la risoluzione dei problemi. L'obiettivo è quello di garantire ai nostri pazienti il rapido riconoscimento della diagnosi, la tempestività delle cure salva-vita, l'appropriato uso dei mezzi - per evitare inutili indagini- ed il miglioramento nel decorso clinico. ${ }^{4}$ Il processo del ragionamento clinico dovrebbe essere affrontato da varie prospettive. ${ }^{5}$ Secondo Patel et al. esso può essere differenziato in quattro fasi: astrazione, abduzione, deduzione e induzione: ${ }^{6}$ i) l'astrazione: corrisponde al processo che dal riscontro di uno o più dati porta ad una conclusione (ad esempio, un valore di emoglobina inferiore a $12 \mathrm{~g} / \mathrm{dL}$ in un maschio adulto viene diagnosticato come 'anemia'); ii) l'abduzione: è il ragionamento attraverso il quale, partendo da alcuni fatti che si vogliono spiegare (premesse), si cerca di individuare una possibile ipotesi che li spieghi (conclusione): ${ }^{7}$ nell'esempio rappresentato è il processo di ragionamento a ritroso che tende a spiegare perché un maschio adulto dovrebbe avere anemia; iii) la deduzione è il processo di verifica dell'ipotesi (ad es. dell'anemia) attraverso la conferma di altri risultati diagnostici attesi: se le condizioni X e Y sono soddisfatte, l'inferenza $Z$ deve essere vera; iv) l'induzione è il processo di generalizzazione di uno o più casi particolari, con cui tentiamo di estendere a regola la sua risultante immediata, al fine di ampliare le nostre conoscenze: da più casi e da più pazienti che mostrano segni e sintomi simili, possono essere create regole generali per spiegare nuovi casi da affrontare. Alcuni dei principali modelli di ragionamento clinico applicati nella pratica sono schematizzati nella Tabella $1{ }^{8}$
Il 'gap' tra teoria e pratica, cultura e competenza e tra mondo reale ed EBM: quale profilo ideale di un internista 'giudizioso'?

$\mathrm{La}$ 'cultura' corrisponde sempre all'effettiva competenza? La Evidence Based Medicine non può di per sé - rappresentare per gli internisti un univoco strumento decisionale, se pretende, trasformandosi da tecnica di analisi a dottrina epistemologica, di 'selezionare ' $\mathrm{i}$ dati attendibili da quelli meno attendibili' per un medico 'dubbioso', che deve affrontare un paziente complesso. ${ }^{9}$ Di fatto esiste un divario tra teoria (conoscenza, evidence based medicine, linee guida) e pratica medica nel mondo reale. ${ }^{10}$ Un medico molto colto può acquisire una grande quantità di cognizioni (sfera cognitiva o del sapere), ma, in assenza di altre qualità operative, può non essere in grado di saper gestire il paziente, tanto più se in presenza di co-morbilità o se vi è la necessità di procedure interventistico-strumentali e/o diagnostiche (sfera del saper fare e del saper essere). Analogamente, un medico può conoscere tutte le linee guida più aggiornate nell'ambito di sua pertinenza, ma non sempre - se non sporadicamente - può applicarle al paziente 'reale' complesso. In sostanza: su quali elementi dovremmo giudicare l'internista come professionista 'valente' e 'giudizioso'? In Medicina Interna è frequente imbattersi in situazioni difficili, in cui vi è incertezza sull'efficacia di un intervento diagnostico-terapeutico e/o delle sue alternative. La presenza di queste 'zone grigie' non dovrebbe determinare la paralisi decisionale, perché la evidence

Tabella 1. Descrizione di alcuni modelli di ragionamento clinico.

\begin{tabular}{|c|c|}
\hline Modelli di ragionamento clinico & Breve descrizione delle caratteristiche salienti \\
\hline 1. Ipotesi deduttiva & $\begin{array}{l}\text { il processo di ragionamento diagnostico si compone in un ciclo di } 4 \text { fasi: i) acquisizione } \\
\text { dei dati; ii) generazione di ipotesi; iii) interpretazione; iv) rivalutazione e decisione finale. }\end{array}$ \\
\hline 2. Pattern recognition & $\begin{array}{l}\text { La diagnosi viene effettuata mediante la rilevazione rapida, non analitica, in riferimento } \\
\text { alla corrispondenza del quadro di presentazione clinica con un modello già } \\
\text { precedentemente acquisito dall'esperienza ed impresso nella memoria del medico. }\end{array}$ \\
\hline 3. Modello di ragionamento clinico 'duale' & $\begin{array}{l}\text { Il modello si basa su modalità di ragionamento sia analitiche che non analitiche. La } \\
\text { presentazione iniziale, se subito riconoscibile, verrà gestita con metodo euristico; se invece } \\
\text { il problema del paziente è complicato, verrà impiegato un sistema analitico più } \\
\text { approfondito, in grado di controllare possibili errori determinati dalle 'prime impressioni'. }\end{array}$ \\
\hline 4. Percorso del ragionamento clinico & $\begin{array}{l}\text { La presentazione iniziale del paziente si concentra su spunti iniziali (dati anamnestici, } \\
\text { sintomi, segni, fattori ambientali, ecc.), che interagiscono tra loro per formare un'ipotesi } \\
\text { iniziale. Il medico ha cercato attivamente le informazioni necessarie per confermare questa } \\
\text { ipotesi, ma deve al contempo considerare possibili contraddizioni emergenti, confutandole } \\
\text { i volta in volta. Se la confutazione fallisce, il medico deve rivedere l'ipotesi originale alla } \\
\text { luce dei nuovi dati e prove. L'ipotesi emergente dovrebbe essere confermata di nuovo. }\end{array}$ \\
\hline 5. Modello integrato di ragionamento clinico & $\begin{array}{l}\text { Le caratteristiche del paziente, insieme alle conoscenze già acquisite del medico, portano } \\
\text { a una rappresentazione iniziale del problema saliente del paziente. Se non emerge subito } \\
\text { la diagnosi devono essere raccolte ulteriori informazioni, con rivalutazione dei problemi } \\
\text { lamentati dal paziente, in un ciclo che continua fino alla diagnosi finale. }\end{array}$ \\
\hline
\end{tabular}

Modificata $\mathrm{da}^{8}$. 
based medicine richiede di fondare le decisioni cliniche sulle 'migliori evidenze disponibili' e non sulle 'migliori evidenze possibili', ovvero praticabili. Proprio sulla base di un adeguato 'ragionamento clinico', il medico internista deve essere in grado di estrapolare, dal problema del paziente, la strutturazione del quesito prevalente, saper ricercare ed individuare la 'best available evidence'e possedere la capacità di valutazione critica della validità e trasferibilità delle informazioni (critical appraisal). Nella valutazione dell'efficacia clinica delle scelte d'intervento l'internista dovrebbe sempre chiedersi se queste funzionano, producendo più effetti positivi che negativi (efficacy), nelle circostanze di pratica clinica abituale (effectiveness) e se 'ne vale la pena', considerando gli effetti prodotti in relazione alle risorse utilizzate (efficiency), ai rischi potenziali (risk management) e ai valori in gioco (Value Based Medicine).${ }^{11}$ Dovrebbe altresì saper effettuare tutte le procedure 'salva-vita', nonché le indagini strumentali/diagnostiche di primo approccio, anche integrate dalla 'bed-side-imaging', per la ridefinizione dell'ipotesi diagnostica iniziale. Dovrebbe infine saper condividere con il paziente tutte le opzioni decisionali, sulla base di valori espliciti e formalmente manifesti.Nel bagaglio di conoscenza dell"internista maturo' dovrebbe essere chiara la differenza tra EBM epistemologica e quella pratica. ${ }^{12}$ Nella prima le evidenze sono il risultato di una conoscenza prevalentemente fondata su elementi probabilistici, acquisita attraverso i trial clinici, a prescindere da ogni altra forma di conoscenza (esperienza, intuizione, autorevolezza clinica, dati fisiopatologici, comorbilità, eccetera). Il principale strumento in questo caso è rappresentato dalla revisione sistematica, in grado di offrire una sintesi di tutta l'evidenza disponibile. Nella EBM applicata alla clinica le due componenti di EBM epistemologica e pratica dovrebbero essere combinate. Porzsolft et al. hanno proposto un modello di processo decisionale a sei 'passaggi':

- scomposizione del quesito clinico in base ai problemi salienti, agli interventi principali dovuti e a possibili interventi alternativi, in funzione degli obiettivi clinici desiderati;

- l'utilizzazione della conoscenza tacita, ovvero di quella conoscenza già acquisita attraverso la formazione professionale e l'esperienza applicata;

- impiego di prove esterne, ottenute con valutazione critica delle fonti (libri di testo, riviste specializzate, banche dati, esperti;

- valutazione del processo decisionale, sulla base di alcuni quesiti rilevanti: i) i risultati sono validi?; ii) i risultati sono clinicamente importanti?; iii) i risultati possono applicarsi al mio paziente? (ovvero: il mio paziente è così diverso da quelli dello studio che i risultati non posso applicarli?);

- le fonti di informazione (esterne e interne) possono essere di aiuto, non supportate da prove o in conflitto. In questi casi la decisione potrà dipendere da più fattori, correlati al contesto e alle opinioni del paziente condivise con il medico;

- dopo la decisione intrapresa si valutano il processo e il risultato, considerando le opportunità di miglioramento in una valutazione epicritica. ${ }^{13}$

\section{Tassonomia delle capacità di pensiero e di ragionamento clinico: le abilità richieste e le sfide}

Per valutare le abilità di ragionamento di livello superiore (higher order thinking skills), dovremmo chiederci quali caratteristiche dovrebbe possedere l'internista per un pensiero multi-logico, definito come 'il modo di pensare per applicare logicamente e sistematicamente $\mathrm{i}$ concetti acquisiti alla soluzione di un problema complesso'.${ }^{14}$ Le abilità dovute in tale ambito possono evolversi da un livello inferiore ad uno superiore, finalizzate secondo una tassonomia più moderna, alla soluzione dei problemi attivi, anche in maniera creativa (Figura 1). ${ }^{15,16}$

Il ragionamento clinico presuppone una solida cultura medica di base ${ }^{17}$ ma dovrebbe soprattutto articolarsi secondo alcuni elementi fondamentali, in relazione alle attitudini mentali, alla narrazione del paziente, al contesto operativo e all'esperienza personale, nonché alla prevalente tipologia strutturale e metodologica su cui il professionista intende basare le sue considerazioni, con un iter sequenziale, fino alla decisione finale) (Figura 2). ${ }^{18,19}$

Secondo il modello di Murtagh il ragionamento clinico dovrebbe imperniarsi su alcune domande fondamentali: i) qual è la diagnosi più i probabile? ii) quali elementi non devono essere trascurati? iii) quali diagnosi alternative possono essere mancate, anche per mascheramento o atipicità dei sintomi presenti? iv) cosa d'altro sta cercando di dirmi il paziente? v) quali sono le opinioni del paziente? ${ }^{20}$ Nel paziente complesso ai quesiti riportati dovrebbero aggiungersene altri su come meglio decidere, ad esempio, nello specifico: per questo paziente, con le co-morbilità associate, in questo contesto assistenziale, con i tempi di attesa e i problemi di accesso esistenti. Inoltre: qual è la cosa più importante e quale è la più pericolosa? Cosa dovrei fare dopo, cos'altro potrebbe succedere? ${ }^{21}$

\section{Attitudini mentali ('habits of mind')}

Quando gli uomini sperimentano dicotomie sono confusi dai dilemmi, faccia a faccia con l'incertezza. L' 'attitudine mentale' è la disposizione a comportarsi in modo intelligente di fronte ai problemi. L' 'abito 


\section{Abilità richieste per livelli cognitivi più elevati secondo la tassonomia di Bloom} Bloom's-Taxonomy

\section{Abilità superiori di ragionamento e di pensiero} HOTS - Higher Order Thinking Skills

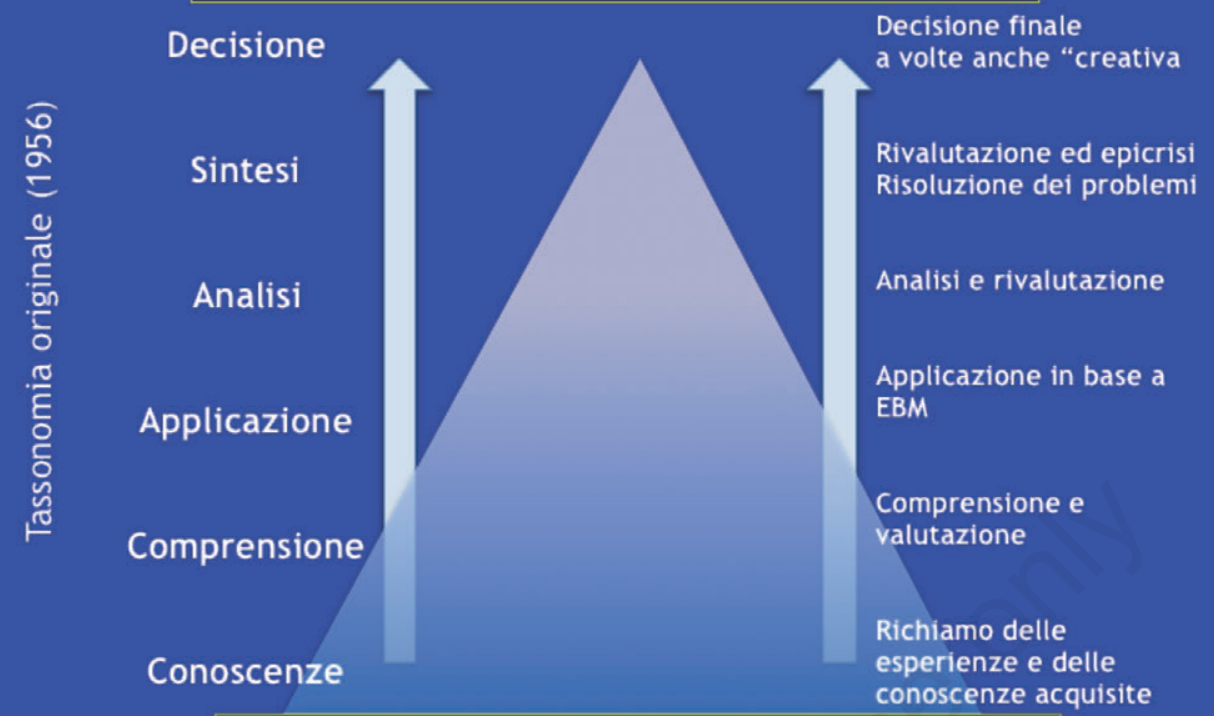
a volte anche "creativa

Rivalutazione ed epicrisi Risoluzione dei problemi esperienze e delle

Abilità inferiori di ragionamento e di pensiero LOTS - Lower Order Thinking Skills

Figura 1. Abilità richieste per livelli cognitivi superiori nel paziente internistico complesso secondo la tassonomia di Bloom. (Modificata da ${ }^{15,16}$ ).

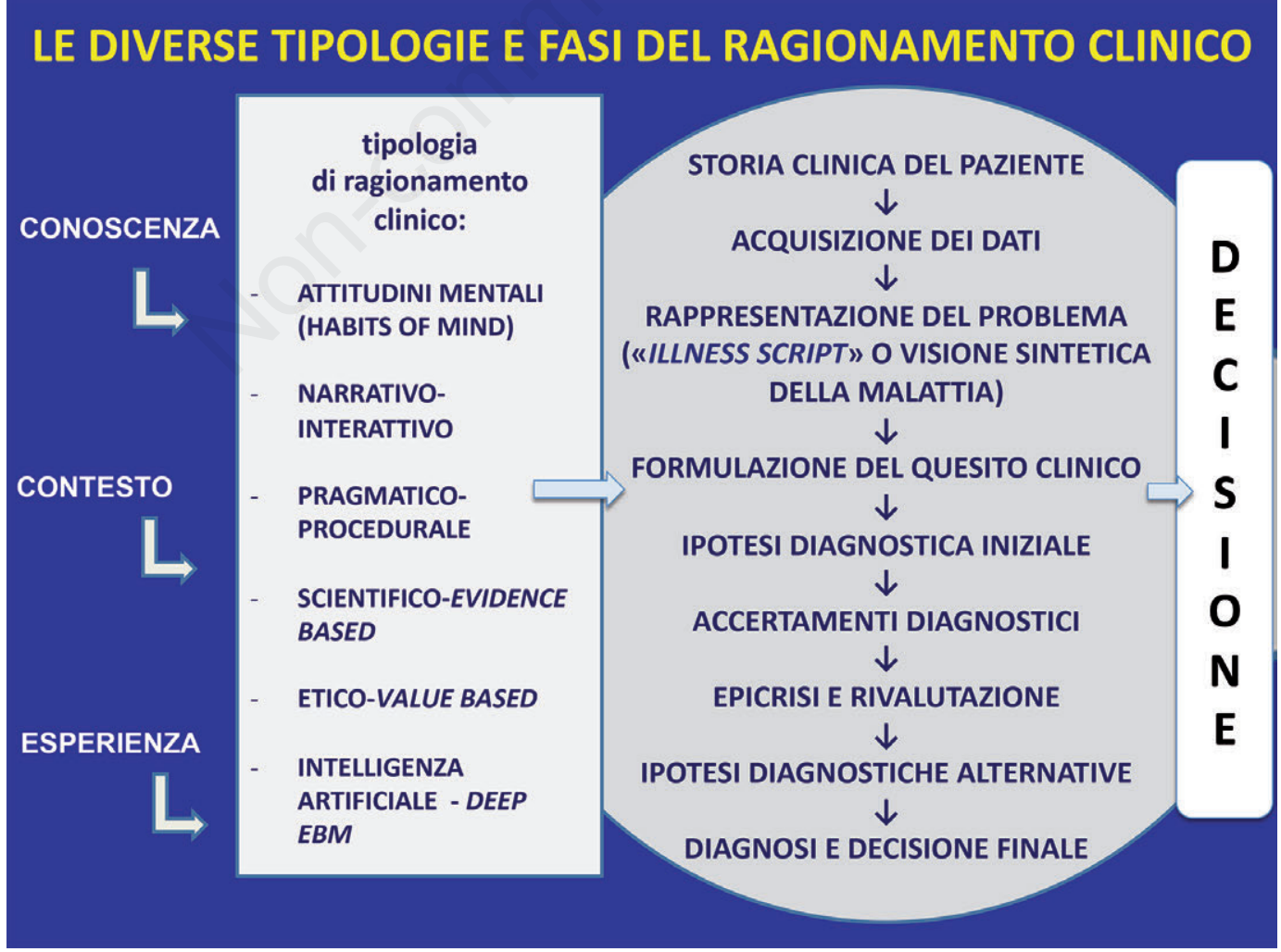

Figura 2. Le diverse tipologie del ragionamento clinico: iter diagnostico per arrivare alla decisione finale. $\left(\mathrm{Da}:{ }^{18,19}\right)$. 
mentale', con le elaborazioni del proprio pensiero, le emozioni e l'esperienza, si basa su diversi attributi: attenzione, capacità critica, saper riconoscere e reagire alle distorsioni cognitive ed emotive, disponibilità a correggere i propri errori. Entrano in gioco diverse caratteristiche individuali, quali la capacità di gestire e tollerare l'ambiguità e l'ansia, l'intelligenza emotiva, il rispetto per i pazienti, il controllo della reattività alle sollecitazioni dell'ammalato e delle sua famiglia, della società e dell'"amministrazione', le capacità empatiche. L'attitudine mentale implica altresì un adeguato livello di consapevolezza dei propri pregi e limiti, ovvero: sapere di sapere, non sapere di sapere, sapere di non sapere o non sapere di non sapere. ${ }^{22} \mathrm{Gli}$ internisti 'esperti', quando devono affrontare problemi le cui soluzioni non sono immediatamente evidenti, attingono a risorse mentali derivate non solamente dal corpo di conoscenze acquisite, ma anche dalla capacità 'superiore' di elaborare un modello di pensiero creativo sulla base delle molte abilità, esperienze pregresse, inclinazioni e sensibilità personali. In questi casi sono richiesti comportamenti intellettuali e azioni efficaci improntati a specifiche abilità, richiamate sinteticamente nella Tabella $2 .{ }^{23}$

\section{Il contesto 'narrativo-interattivo'}

La malattia non è solamente uno stato patologico oggettivabile, misurabile, classificabile, ma anche malessere e sofferenza soggettiva, spesso espressione di un anomalo rapporto tra individuo e ambiente. ${ }^{24} \mathrm{La}$ relazione tra medico e paziente è di tipo fiduciario. Il processo decisionale si sposta dal curante all'assistito, che deve manifestare le sue aspettative, sulla base di informazioni esplicite e condivise. Vale il principio dell' 'alleanza terapeutica', fondata su un processo di comunicazione solidale tra medico e paziente. La medicina narrativa presta attenzione alla storia della malattia, allo scopo di comprendere le patologie dei pazienti e il loro vissuto. La narrazione consente di porsi in una posizione di 'ascolto' (ascoltare è già di per sé un atto terapeutico), di valutare, insieme al paziente, la successione cronologica degli eventi e di comprendere lo stato d'animo (e di salute) del pa-
ziente.In tale ambito il medico dovrebbe possedere competenze specifiche, quali: i) capacità testuali: saper identificare la struttura della storia clinica e riconoscere metafore, allusioni, sfaccettature; ii) capacità creative, su come immaginare diverse interpretazioni della narrazione; iii) capacità affettive ed interattive, su come saper entrare in maniera empatica nella storia riferita dal paziente. ${ }^{25} \mathrm{~L}$ 'empatia si realizza nella capacità di capire in maniera speculare una persona, attraverso l'immedesimazione, il pensiero e la percezione, rispetto ai nostri modi di vedere, comprendendone i sentimenti, le idee e le determinazioni soggettive, a partire dal contesto dell'altro (status socio-economico e famigliare, cultura, appartenenza di classe) e dalle valutazioni clinico-prognostiche. ${ }^{26}$ Una schematica rappresentazione comparativa tra pensiero creativo e pensiero critico che si compie nei diversi passaggi del ragionamento clinico, con relative capacità di tipo cognitivo/affettivo, è riportata nella Figura $3 .{ }^{27}$

\section{Il metodo 'scientifico'- EBM: utile per la formulazione del quesito clinico}

Quando dobbiamo assumere decisioni abbiamo bisogno di chiederci - anche implicitamente - quale è il quesito clinico principale, in relazioni ai dati disponibili, al contesto e alle possibili decisioni che potremmo assumere (Tabella 3 ).

Il metodo scientifico 'Evidence based Medicine' (EBM) è importante per lo studio dei determinanti che intervengono nelle decisioni cliniche. L' EBM consente di superare, in presenza di prove documentate, possibili pregiudizi decisionali, correlati ad intuizioni cliniche automatiche, autoreferenziali e non sempre appropriate. Esso si basa su una serie di passaggi che ogni singolo professionista dovrebbe porsi in maniera sistematica: i) chiediti, ovvero formula la domanda cruciale ('il quesito clinico') sul problema da risolvere; ii) acquisisci e contestualizza i dati disponibili; iii) acquisisci le evidenze e cerca risposte in merito; iv) valuta le evidenze per qualità e rilevanza e applicabilità; v) applica le indicazioni suggerite dalle prove; vi) valuta i risultati.Uno

Tabella 2. Elementi fondanti le attitudini mentali.

\begin{tabular}{ll}
\hline Item & Abilità di: \\
\hline Valore & scegliere di utilizzare un modello di comportamento intellettuale piuttosto che altri modelli meno proficui \\
\hline Inclinazione & percepire la tendenza a utilizzare un modello di comportamento intellettuale \\
\hline Sensibilità & saper cogliere le opportunità ed utilizzare un modello di comportamento piuttosto che un altro. \\
\hline Capacità & portare a termine gli obiettivi desiderati con il comportamento intrapreso. \\
\hline Impegno & lottare costantemente per migliorare le prestazioni del modello di comportamento intellettuale \\
\hline
\end{tabular}

Fonte: ${ }^{23}$. 
strumento utile da richiamare ai fini della formulazione del quesito clinico è il 'metodo PICO$\mathrm{T}$ ' ${ }^{28,29}$ L'acronimo originale deriva dai seguenti elementi: la valutazione del Paziente e dei Problemi da risolvere (Patient or problem to solve), l'Intervento da implementare (Intervention), il confronto tra risultati attesi e quelli ottenuti (Comparison), dei risultati (Outcome), cui si è poi aggiunto il Tipo di scenario (Type of scenario-setting). Altre varianti sono state successivamente introdotte rispetto al format originario del PICO, quali PICOM, PICOS, PICOT, EPICOT + , PESICO, PICOTT, PECODR, eccetera) nei diversi ambiti dell'area sanitaria e sociosanitaria. ${ }^{30}$

\section{La 'real world evidence' medicine (RWEM) in confronto con la EBM, basata sui trials clinici randomizzati (RCTs)}

Esistono differenze sostanziali tra il punto di vista del 'metodologo EBM' e il clinico, più orientato ad una pratica applicata al mondo reale (Figura 4).

L'EBM pragmatica tende a descrive come praticare in modo ottimale la medicina attraverso strumenti aggiuntivi, quali le linee guida, gli studi di real word evidence e di audit clinico per il raggiungimento degli standard desiderati. Il tema dell'evidenza nel mondo reale è quello posto dalla 'real world evidence medicine (RWEM)'. ${ }^{31}$ I dati del

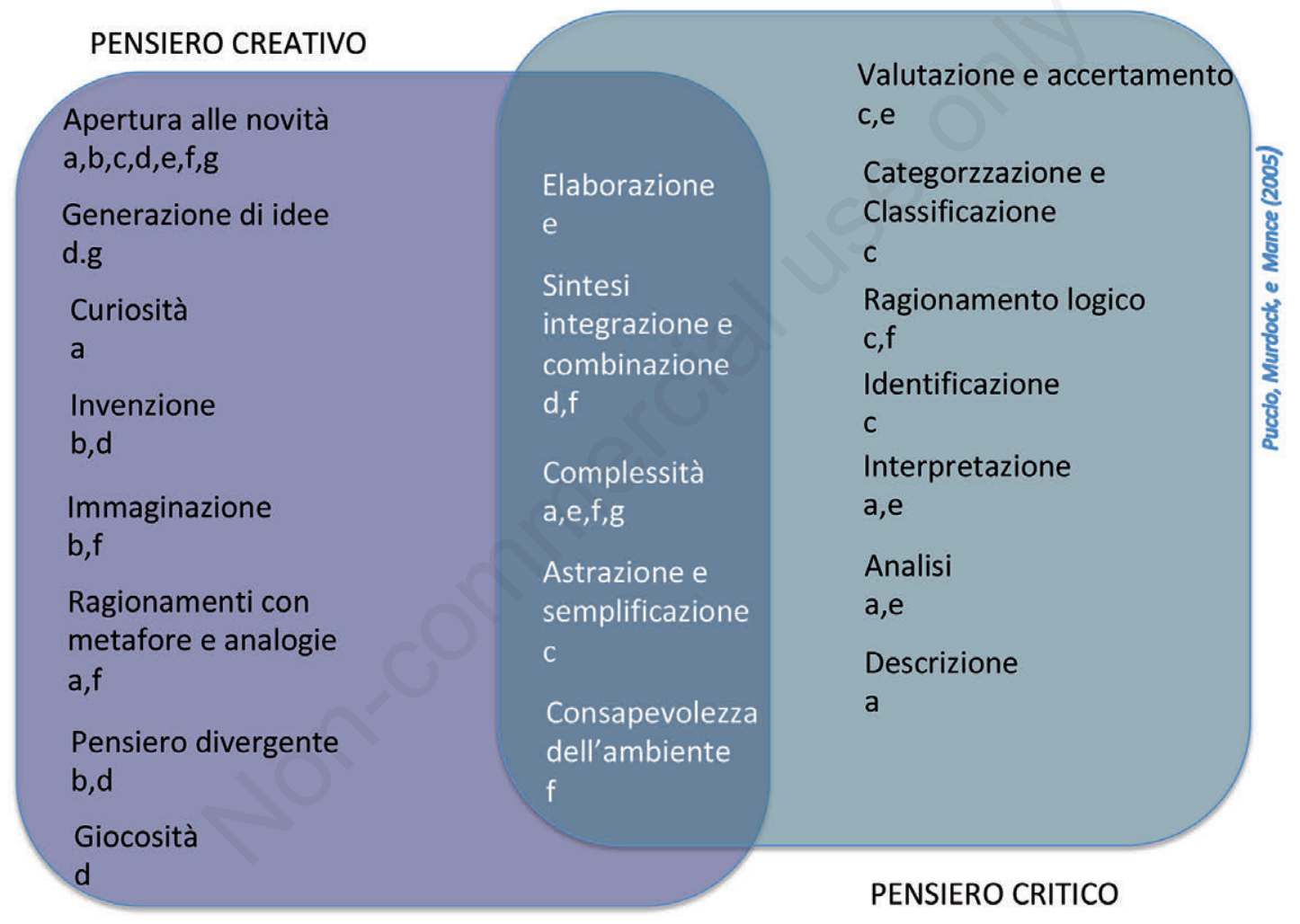

\begin{tabular}{|c|c|c|c|c|c|c|}
\hline \multicolumn{7}{|c|}{ Step } \\
\hline a & b & c & d & $\mathrm{e}$ & $f$ & g \\
\hline \multicolumn{7}{|c|}{ Capacità cognitive } \\
\hline \multicolumn{7}{|c|}{ Capacità affettive } \\
\hline Curiosità & Sognare & $\begin{array}{l}\text { Colmare le } \\
\text { lacune }\end{array}$ & Giocosità & $\begin{array}{l}\text { Evitare chiusure } \\
\text { premature }\end{array}$ & $\begin{array}{l}\text { Sensibilità } \\
\text { all'ambiente }\end{array}$ & $\begin{array}{l}\text { Tolleranza ai } \\
\text { rischi }\end{array}$ \\
\hline
\end{tabular}

Figura 3. Pensiero creativo e pensiero critico. (Modificata $d a^{27}$ ). 
mondo reale (Real-World Data- RWD) sono basati sui database elettronici sanitari acquisiti da registri di malattia o da studi osservazionali e rappresentano uno dei temi più discussi nell'ambito dei congressi medici. ${ }^{32}$ Le differenze tra la EvidenceBased
Medicine (EBM), basata sui trial clinici randomizzati (RCTs-randomized controlled trials) si differenzia dalla Real World EvidenceMedicine per alcune caratteristiche fondamentali, schematizzate nella Tabella 4.

Tabella 3. Il quesito clinico e le decisioni: le domande da farsi.

\section{IL QUESITO CLINICO:}

- Il quesito che mi pongo è chiaro, comprensibile, corretto e congruente con i dati a mia disposizione?

- Il quesito che mi pongo è importante per il paziente?

- Il quesito che mi pongo dovrebbe essere scomposto secondo sotto-quesiti parziali?

- Come posso sinteticamente formulare il quesito clinico che mi pongo per esplicitarlo in maniera chiara?

\section{IPOTESI DECISIONALI:}

- La mia decisione si sta orientando per quale ipotesi diagnostica?

- Quali altre alternative si potrebbero porre e perché?

- Questa ipotesi è congruente con i dati contestuali?

\section{IL PUNTO DI VISTA:}

- Ho considerato il punto di vista del paziente?

- Quale prospettiva mi ha indotto ad orientarmi nell'ipotesi diagnostica formulata?

- Cosa direbbe qualcuno che non è d'accordo con il mio punto di vista?

- Vi sono prospettive alternative al mio modo di vedere?

\section{EVIDENZE E MOTIVAZIONI:}

- Quali sono nella mia ipotesi gli elementi che garantiscono un risultato di qualità e di sicurezza per il mio paziente?

- Quali evidenze posso addurre per la mia ipotesi?

- Quali accertamenti diagnostici sono necessari e quali invece potenzialmente inappropriati?

- C'è qualche motivo per dubitare delle evidenze che ho portato?

- Quali elementi critici potrebbero inficiare l'ipotesi?

IMPLICAZIONI E CONSEGUENZE:

- Che effetto avrà la mia decisione?

- Vi sono possibili errori nella mia ipotesi?

- Qual è la probabilità che sia effettivamente verificata come corretta?

- Quali sono le possibili alternative?

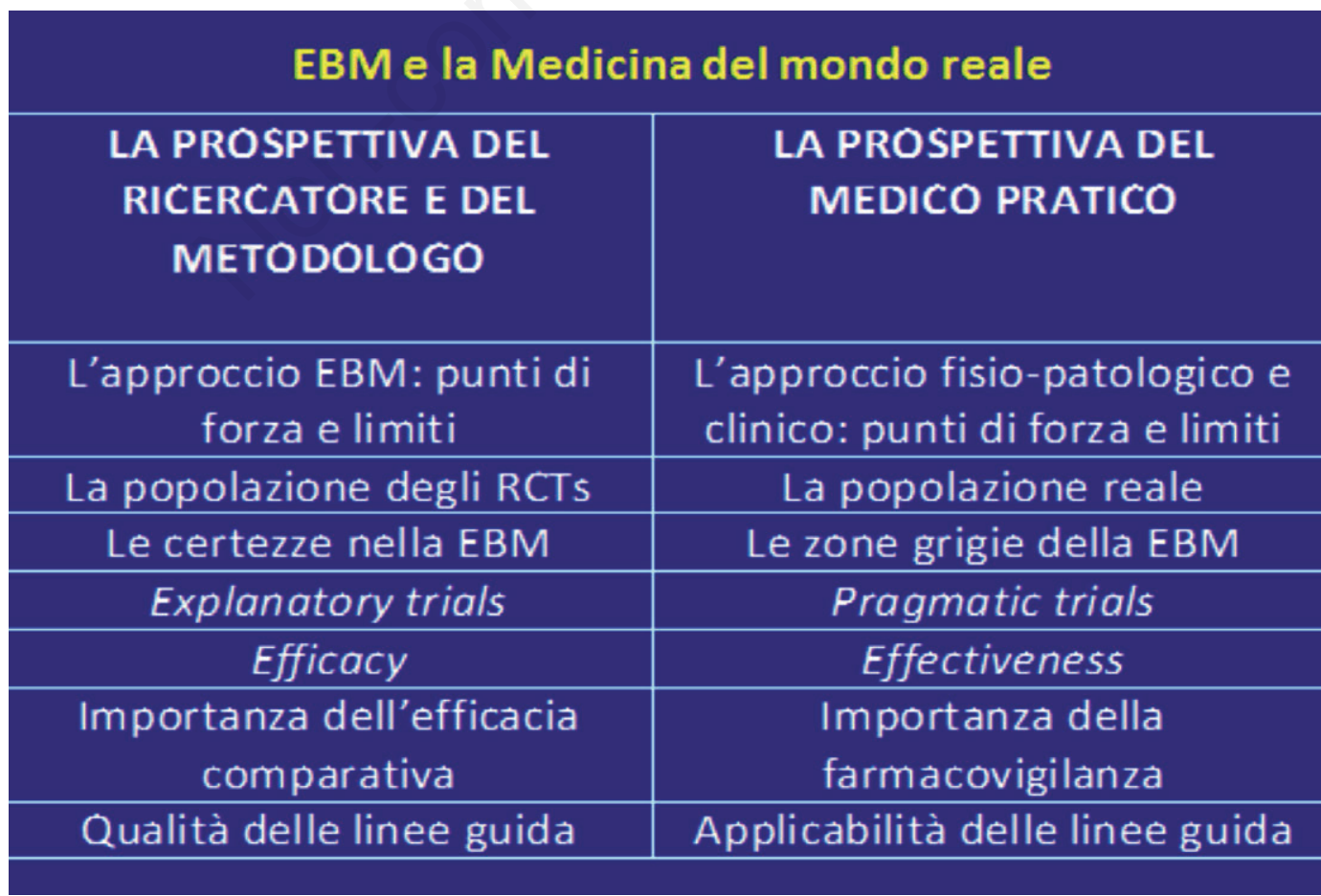

Figura 4. EBM e la Medicina del mondo reale. 


\section{La Knowledge based medicine (KBM)}

L' identificazione, l'acquisizione, la registrazione e la trasmissione delle conoscenze fra i diversi specialisti della salute sono fondamentali. Studiando le conoscenze disponibili in un sistema sanitario si può accedere alle vare esperienze cliniche individuali e alle capacità intuitive di problem solving dei professionisti (conoscenza tacita) e alle informazioni strutturate fruibili, quali linee guida, studi, recensioni basati su evidenze o esperienze personali (conoscenza esplicita). La mappa della conoscenza può essere finalizzata anche alla formazione dei professionisti, sulla base del risconto di elementi di conoscenza mancanti o carenti. La valutazione delle conoscenze disponibili nell'organizzazione potrà altresì evitare l'uso eccessivo, il sottoutilizzo e l'uso improprio delle cure. Questa visione della KBM deve essere differenziata da altri due elementi confondenti: i) i cosiddetti 'sistemi basati sulla conoscenza' o 'esperti', ovvero programmi informatici progettati per risolvere problemi, generare nuovi informazioni (ad esempio, anche una diagnosi) o fornire consigli, usando una base di conoscenza e meccanismi inferenziali; ${ }^{34}$ ii) una Knowledge Based Medicine o medicina basata sulle conoscenze, orientata ad una visione fisiopatologica delle malattie, in cui il processo decisionale è così delicato e complesso da non potersi limitare a mera applicazione di 'linee-guida' o protocolli, ispirate ad un paziente 'ideale', in un'unica 'categoria di cura' e in assenza di altre co-morbilità interferenti. Questo, al contrario, dovrebbe essere finalizzato a personalizzare l'intervento medico sul singolo paziente, ancorando gli interventi alle conoscenze disponibili, superando una concezione 'ingessata' della medicina basata sulle evidenze, approdando, invece, a quella più dinamica di una medicina basata sulle conoscenze disponibili. ${ }^{35}$

\section{L'approccio VBM (Value Based Medicine)}

L'approccio VBM (Value Based Medicine) integra la EBM (EvidenceBased Medicine). Il valore degli interventi sanitari dipende dai risultati clinici e dal miglioramento della qualità della vita ottenuti, in base ai costi globali ad esso correlati, non dal volume dei servizi erogati. Tali elementi andrebbero sempre definiti insieme ai pazienti. ${ }^{36}$ Secondo Sir MuirGray vi sono tre categorie di valore: i) il valore personale, basato sulla fondamentale centralità delle attese del paziente nel processo assistenziale; ii) il valore tecnico, inteso come miglior risultato tra l'esito delle cure e l'investimento sostenuto); e iii) il valore allocativo, ovvero il confronto dell'impatto di diversi possibili interventi sanitari attuabili con le stesse risorse. ${ }^{37} \mathrm{La}$ 'crisi' della EBM richiede un richiamo ai suoi principi fondanti, sulla base di un rinnovato contesto umanistico e professionale che regola le relazione tra medico e paziente. ${ }^{38}$ In sostanza, 'la medicina delle prove' (EBM) e quella dei 'valori'(VBM) confermano la necessità prioritaria del coinvolgimento del paziente nelle decisioni cliniche assunte, in sintonia con le evidenze disponibili, le sue decisioni ed aspettative e i suoi valori. ${ }^{39,40}$ La VBM integra la miglior EBM con la qualità percepita dal paziente sul miglioramento arrecato da un intervento sanitario anche in rapporto ai costi e alle risorse investite per evitare qualsiasi spreco ${ }^{41}$ Per garantire la sostenibilità del SSN, i professionisti sanitari devono prendere coscienza che una quota rilevante di 'inappropriatezze' deriva proprio da un sovra-utilizzo di interventi e di prestazioni sanitarie..$^{20,42}$ L'obiettivo dell'assistenza sanitaria dovrebbe perseguire un 'esito di salute' compatibile con la sostenibilità economica del sistema sanitario.

Tabella 4. Differenze tra RCTs e RWE (Randomized Controlled Trials vs Real World Evidence). ${ }^{33}$

\begin{tabular}{|c|c|}
\hline EBM_RCTs & RWEM \\
\hline $\begin{array}{l}\text { Danno informazioni prevalentemente sull'efficacia e la sicurezza } \\
\text { dei farmaci }\end{array}$ & $\begin{array}{l}\text { L'efficacia e la sicurezza dei farmaci vengono valutate nell'assistenza } \\
\text { routinaria }\end{array}$ \\
\hline Durata e follow up (FU) relativamente brevi & $\begin{array}{l}\text { FU e uso prolungato dei farmaci con possibilità di verificare nel lungo } \\
\text { termine sicurezza e reazioni avverse rare }\end{array}$ \\
\hline Ottima compliance alle terapie da parte dei pazienti & Compliance incerta e necessità di ottenere dati di buona qualità \\
\hline La randomizzazione tiene conto dei possibili fattori confondenti & $\begin{array}{l}\text { Disegno e analisi statistica studiati per minimizzare l'influenza di fattori } \\
\text { confondenti }\end{array}$ \\
\hline \multicolumn{2}{|l|}{ ma: } \\
\hline $\begin{array}{l}\text { Popolazione degli studi ristrette e selezionata (basso rischio, } \\
\text { scarsa validità esterna) }\end{array}$ & Informazioni limitate sui fattori confondenti o esclusione degli stessi \\
\hline $\begin{array}{l}\text { Confronto generalmente con un comparatore (quello impiegato } \\
\text { comunemente o un placebo) }\end{array}$ & Confronto fra diversi trattamenti dusoinibili \\
\hline Non in grado di riflette la pratica clinica reale & $\begin{array}{l}\text { Analisi statistica complessa integrata dalla valutazione della } \\
\text { significatività clinica }\end{array}$ \\
\hline
\end{tabular}




\section{L'approccio basato sull'intelligenza artificiale (DEEP EBM): un futuro già attuale?}

Eric Topol, insigne cardiologo e fondatoredirettore del ScrippsTranslational Science Institute, tra i massimi esperti al mondo di digital health, sostiene che l'innovazione tecnologica (genomica, medicina digitale, telemedicina, intelligenza artificiale e robotica) potrà cambiare i ruoli e le funzioni del personale clinico di tutte le professioni nel prossimo futuro, facilitando l'umanizzazione delle cure con una medicina più orientata ai cittadini. ${ }^{43}$ I futuri professionisti della salute potranno ottimizzare le capacità di comunicazione, monitoraggio, ricerca, collaborazione e giudizio, affrontando i problemi con tecnologie innovative, che potranno consentire di disporre di una grande quantità di dati, oltre che di più tempo per utilizzare le capacità cognitive e garantire maggiore spazio al rapporto con il paziente, considerando che oggi una gran parte del lavoro è speso per processi amministrativi.

\section{Intelligenza artificiale: algoritmi e decisioni mediche, pro e contro}

L'impiego di mezzi informatici ha avuto un notevole sviluppo negli ultimi anni in ambito sanitario, con la creazione di applicazioni mirate a supportare le decisioni cliniche (Clinical Decision Support o CDS). L'onda della tecnologia digitale è tuttora in rapida evoluzione e può virtualmente offrire l'opportunità di assumere decisioni sulla base delle evidenze scientifiche, attraverso l'immissione di una considerevole quantità di dati ('big data'), solidi e documentati, con relative analisi predittive, a fronte della 'semplice' esperienza individuale dei singoli professionisti. ${ }^{44}$ Tali strumenti hanno oggi impieghi assai variegati, ad esempio, per la gestione dei dati di laboratorio e microbiologici, per la diagnosi precoce e la prevenzione di diverse malattie in ambito epidemiologico, a supporto della gestione della terapia anticoagulante orale, per la gestione della sepsi e la predizione della mortalità correlata, per monitorare gli eventi infettivi in ospedale, nella prescrizione di oppiacei, nella gestione del diabete, per la riconciliazione, la sicurezza e l'efficacia della terapia farmacologica in regimi terapeutici complessi, per la gestione delle cartelle cliniche elettroniche e l'estrazione di dati da queste, per fornire le giuste informazioni ai pazienti affetti da malattie croniche, accompagnandoli nel loro processo di cura. Per facilitare le scelte diagnostico-terapeutiche sono stati proposti diversi algoritmi, con relativi passaggi su come affrontare segni, sintomi, problemi e dati di laboratorio in vari contesti clinici. Sono finalizzati a guidare il pensiero clinico e i processi decisionali, anche per ridurre al minimo gli accertamenti non necessari, controllare i costi e fornire un'assistenza di qualità. ${ }^{45}$ I sistemi di supporto decisionale aiutano i clinici nel prendere decisioni, ma, a parte i possibili limiti intrinseci di tali strumenti, in alcuni casi non riescono a considerare importanti informazioni contestuali, ${ }^{46}$ particolarmente di quelli rappresentati nell'analisi narrativa dei problemi. Gli algoritmi proposti possono essere pertanto strumenti a 'doppio taglio', in quanto, sfruttando modelli basati su molti dati disponibili, possono a volte portare a decisioni imperfette. ${ }^{47}$ I vantaggi di queste nuove tecnologie dovrebbero prevalere sui loro possibili limiti, quali, ad esempio: la scarsa facilità d'impiego, troppo tempo applicativo necessario per flussi di lavoro già sotto forte pressione, il rischio d'inerzia di giudizio da parte dei sanitari ed una possibile scarsa garanzia di informazione, la riservatezza dei dati e di privacy per i pazienti. I sistemi che utilizzano algoritmi decisionali hanno ovviamente un potenziale molto appetibile per i gestori, interessati a migliorare l'efficienza e ridurre i costi, ma possono influire negativamente sui diritti umani -individuali o collettivi- tali da richiedere un controllo normativo. ${ }^{48}$ Inoltre, cosa succede quando sulla base di un algoritmo viene presa una decisione distorta? Come decidiamo chi dovrebbe assumersi la responsabilità? I metodi con cui le decisioni vengono attuate devono essere trasparenti, in modo da poter personalmente 'rendere conto' sulle decisioni assunte se qualcosa dovesse andare storto. ${ }^{49}$ L'arte della medicina si basa sul giudizio del medico, in una sinergica e stretta relazione con il suo paziente e non solamente sui dati formali disponibili: il bilanciamento di questi due elementi non è sempre semplice..$^{50}$ L'abilità cognitiva, emotiva, interattiva, interpretativa e creativa del medico non è necessariamente un limite all'implementazione di strumenti informatici, ma, al contrario, rappresenta il valore aggiunto apportato dall'uomo agli utili 'artificiali, sistemi intelligenti', a garanzia di giuste e responsabili decisioni nella relazione personale col paziente.

\section{Conclusioni}

Di fatto l'internista applica, anche in maniera implicita e contemporanea, diverse tipologie di ragionamento clinico, tra quelle descritte. Le 'abilità richieste' riguardano diversi ambiti, di seguito rappresentati.

\section{Conoscenza, esperienza e capacità di richiamare la propria storia professionale al caso attuale}

Le migliori evidenze disponibili sono solamente uno dei fattori su cui basare le decisioni cliniche: in queste vengono coinvolti altri elementi importanti quali l'esperienza del clinico, le preferenze del 
paziente e le opportunità organizzative. Tra gli elementi costitutivi della competenza, alcuni riguardano la natura del lavoro e possono essere individuati analizzando le attività svolte; altri invece (come motivazione, capacità di comunicazione, capacità di problem solving) hanno a che fare con caratteristiche 'personali' e la storia professionale del soggetto, che entrano in gioco nel contesto operativo. ${ }^{51}$ L'esperienza innesca di per sé un 'apprendimento implicito' e contribuisce al miglioramento e al consolidamento della competenza, inducendo relativi comportamenti.

\section{Comprensione, valutazione selezione dei bisogni e delle priorità: l'importanza delle mindlines}

Il compito essenziale dell'internista è quello d'impostare l'iter diagnostico su un orientamento mirato alla selezione delle necessità attuali. Nella selezione dei problemi attivi si rende necessaria una gerarchizzazione delle priorità, iniziando, ad esempio, dalle possibili condizioni gravate dall'immediata minaccia alla vita del paziente, ovvero dalla necessità di assumere provvedimenti urgenti. Dovrebbe essere impostata una diagnosi differenziale, con l'elencazione di possibili diagnosi alternative per l'interpretazione del problema, tenendo conto del fatto che liste con un numero elevato di problemi (ad esempio, oltre 10), possono evocare una paralisi decisionale. Devono pertanto essere eliminate progressivamente tutte le opzioni diagnostiche prive di fondamento, ricorrendo non solamente alle linee guida ma anche al bagaglio di conoscenze tacitamente acquisite dalla comunità clinica (le cosiddette 'mindlines'), derivanti da letture varie, dall'esperienza, dal confronto con altri professionisti, esperti, società Scientifiche, aziende farmaceutiche e gli stessi pazienti. ${ }^{52}$

\section{Applicazione dell'EBM: messa in atto dell'iter diagnostico-terapeutico in base al quesito clinico}

La conformità dei comportamenti professionali dovrebbe essere definita dagli standard di competence professionale (in particolare le skills), che rappresentano il 'buco nero' della qualità assistenziale. ${ }^{53} \mathrm{Il}$ medico, oltre al bisogno d'informazione su come formulare adeguati quesiti clinici, non dovrebbe limitarsi a ricevere passivamente indicazioni operative, ma dovrebbe saper collegare le conoscenze acquisite in un contesto integrato di cultura sempre più ricco (semantic network), in grado di 'trasformare l'informazione in conoscenza'. Nella EBM si possono evidenziare tre componenti, ciascuna delle quali richiede strumenti e competenze (tecniche, logiche ed umanistiche), che raramente fanno parte dei tradizionali curricula formativi.
- La prima, 'essenziale', richiede al medico: i) di migliorare il 'bisogno d'informazione', allo scopo di ottimizzare la conoscenza emersa dall'incontro con il paziente; ii) di convertire tale bisogno in quesiti clinico-assistenziali ben definiti.

- La seconda, 'tecnica', consente al medico di trovare con la massima efficienza le migliori evidenze disponibili in letteratura e di interpretarle criticamente nella loro validità interna ed esterna.

- La terza, strettamente correlata al giudizio clinico (clinical judgment), consente di valutare, in modo ponderato, l'importanza e l'applicabilità di tali evidenze ai fini della decisione clinica, tenendo conto delle preferenze ed aspettative del paziente, ed anche del contesto sociale, organizzativo ed economico. ${ }^{54}$

L'esperienza del singolo professionista, in sostanza, costituisce l'insieme di capacità professionali e giudizio clinico, fondamentale per comprendere se le evidenze raccolte siano applicabili alla persona malata, in ogni loro aspetto, in parte o affatto. La EBM non deve essere identificata con le linee guida (LG) ${ }^{55,56}$ La maggior parte delle 'linee guida' affronta malattie tipiche dell'età avanzata, ma poche di esse riguardano la popolazione anziana con co-morbilità. Ciò deriva da un'impostazione delle LG più basata sulle singole malattie che sui pazienti reali. ${ }^{57}$ In particolare le Evidence-Based Guidelines costituiscono solamente una componente della EBM, che è improntata ad un rinnovamento culturale finalizzato a modificare le modalità di apprendimento del medico e il trasferimento delle conoscenze acquisite nella pratica clinica. ${ }^{58}$

\section{Analisi, sintesi, rivalutazione e punto della situazione: l'importanza dell'epicrisi}

L'epicrisi (dal greco epi- 'sopra' e krisis 'decisione, giudizio') è una sintesi critica e/o analitica del caso clinico, che implica una riflessione sul decorso della malattia e sulla sua gestione. Essa dovrebbe essere un momento fondamentale nell' approccio metodologico ai problemi del paziente e non solamente un ulteriore riquadro da compilare per la qualità documentale/formale della cartella clinica. La revisione sintetica dei dati disponibili dovrebbe essere finalizzata a rivalutare complessivamente il caso e a riorientare il procedimento diagnostico se il nodo della diagnosi non è stato ancora risolto.

\section{L'integrazione del ragionamento clinico con le capacità gestionali e comunicative}

Una buona comunicazione tra medico e paziente è finalizzata ad acquisire non solamente $\mathrm{i}$ dati anamnestici essenziali, ma soprattutto ad ottenere, le informazioni riguardo all'illness, su come il paziente 
percepisce la malattia, ciò che lo preoccupa in riferimento all'esperienza personale e al vissuto del proprio stato di salute, in un rapporto di reciproco ascolto empatico. ${ }^{59}$ Una comunicazione efficace migliora la relazione terapeutica fra medico e paziente e consente di superare il gap fra EBM e le attese individuali degli ammalati, facilitando le decisioni in condizioni di incertezza, migliorando il grado di soddisfazione del paziente e contribuendo ad una migliore professionalità del medico. ${ }^{60}$

\section{Decisione finale, anche di tipo creativo, in un approccio antropologico al paziente}

Il quesito clinico evidentemente condiziona le decisioni finali, sulla base della generazione di una diagnosi differenziale, con la valutazione ponderata delle possibili diagnosi alternative per interpretazione del problema in causa. In questo processo il medico si basa sulle conoscenza scientifiche, ma a volte deve operare in una dimensione extra-scientifica interpretativa e di meta-cognizione, ricorrendo anche al pensiero laterale, all'intelligenza emotiva, alla capacità creativa nell'immaginare un'alternativa diagnostica più attendibile di quelle immediatamente ipotizzate.

\section{Medicina, conoscenza e ragionamento clinico: arte o scienza?}

\author{
'Con innocenza e purezza io custodirò \\ la mia vita e la mia arte. \\ Ippocrate, Giuramento, (460-375 A.C.)
}

'Scientia potentia est', sapere é potere e, ancora, di Bacone: 'nam et ipsa scientia potestas est ${ }^{61}$ poiché la scienza è di per sé una potenza, algoritmo virtuoso che permette di utilizzare al meglio ogni paradigma della conoscenza che automaticamente diventa chiave indispensabile di ogni disciplina. ${ }^{62}$ Qualcuno sostiene che 'la medicina non è scienza, bensì un'arte ben realizzata' ("Medicine is not science; it is a well crafted art'), ${ }^{63}$ proprio per descrivere il difficile compito del medico, chiamato a riflettere e a decidere su un malato complesso. SirWilliam Osler sosteneva che 'La medicina è una scienza dell'incertezza e un'arte di probabilità'. ${ }^{64} \mathrm{Se}$ la scienza e l'arte nell'esercizio della medicina sono complementari, si può asserire che un medico dovrà essere per certi versi un artista che si 'agita' in conformità a conoscenze scientifiche. ${ }^{65-67}$ Entro i copioni rivelati dalla scienza si dovrà comprendere e ricostruire un vissuto come atto che ha in sé le stigmate del momento immaginifico e creativo, teso a una riproduzione mimetica proprio dell'arte (mimesi). Quella che oggi nel teatro clinico è definita real life non può essere costretta in un'icastica (mimesi icastica) rappresentazione entrando in arduo conflitto con l'idea rappresentativa di una realtà inesistente (il copione cosiddetto scientifico). ${ }^{68}$ Né appare come una concezione fantastica, si potrebbe dire fumosa o astratta (mimesi astratta), quella che più potrebbe aiutare la mano dell'artista medico nell'intravedere ed interpretare il vero che con sortilegio dispettoso si offre alla sua intelligenza emotiva in quel gioco di luce ed ombre che sempre caratterizza l'entusiasmante e pur difficoltoso momento creativo. ${ }^{69}$ Perché il vero possa prendere corpo nel cammino esistenziale della quotidianità creativa dell'artista medico dovrà necessariamente seguire vie diverse da queste descritte ed anche lo stesso profilo interpretativo del concetto di arte va meglio definito. Se infatti la capacità imitativa si concepisce come una facoltà tecnica, l'arte diventa perizia. Quando la capacità imitativa diventa partecipazione (metessi) l'artista medico si trova d'incanto ad essere strumento interpretativo del reale che ha di fronte ed il vero prende corpo. ${ }^{70}$ Nessuna metodologia clinica potrà dunque prescindere dal rapporto emozionale di partecipazione (empatia), ${ }^{71}$ che lega l'autore/medico al suo paziente/pubblico. Se questo rapporto non si crea, l'aspetto artistico, necessario e complementare a quello scientifico, lascia lo spazio a quel copione rivelato dalla scienza quale idea rappresentativa di una realtà inesistente. Anche secondo Giorgio Cosmacini 'La medicina non è una scienza': è una pratica basata su scienze - la fisica, la chimica, la biologia, l'ecologia, l'economia - che differisce dalle altre tecniche perché il suo oggetto è un soggetto: l'uomo. ${ }^{72}$ L'arte medica può fornire il processo interpretativo per la risoluzione di situazioni incerte, identificando quali informazioni sono mancanti e riconoscendo che l'interpretazione è un processo che si attua col tempo, approfondendo i vari aspetti del contesto. ${ }^{73}$ Questa 'arte' non può essere soffocata dalla burocrazia, ma deve essere facilitata da una sensibilità sociale e da una cultura umanistica tale da poter supportare il paziente nelle sue difficoltà, con un approccio antropologico, in un mondo reale e non utopico. In sostanza, dobbiamo sforzarci nel perseguire una medicina contrassegnata da competenza, ma anche da sentimento. ${ }^{74}$ I medici fanno uso della scienza, ma la medicina non è essa stessa una scienza: è piuttosto una pratica interpretativa che si basa sulla capacità di ragionamento clinico. ${ }^{75} \mathrm{La}$ consolidata 'visione globale' del medico internista è importante. Proprio per evitare derive autoreferenziali, abbiamo bisogno di ulteriori studi ed evidenze, allo scopo di capire quali migliori strumenti di valutazione delle capacità di ragionamento clinico possono essere oggi proponibili in maniera efficace.

\section{Post scriptum}

Nel manoscritto abbiamo voluto mantenere diversi termini inglesi originali 'virgolettati' o in corsivo non 
per una auspicata anglofilia o per ripudio della lingua italiana. La lingua inglese domina la letteratura scientifica medica di qualità. Abbiamo voluto facilitare la lettura delle principali parole chiave sul tema e dei molti articoli di riferimento, con un glossario pertinente all'argomento affrontato.

\section{Bibliografia}

1. Hawkins D, Elder L, Paul R. Clinical Reasoning, The Foundation for Critical Thinking, 2010.

2. Mc Donald MB, McGregor RS, Bhatia D et al. A Milestones Friendly Clinical Reasoning Assessment Tool, Academic Pediatrics 2013, 13/4, e1.

3. Young M, Aliki T, Lubarsky S, Ballard T et al. Drawing Boundaries: The Difficulty in Defining Clinical Reasoning, Academic Medicine 2018, 93/7: 990-995.

4. EuropeanHeartAssociation, Cosa è il Ragionamento Clinico e Perché è Importante?, Post in primo piano, 23 novembre 2017, http://webcache.googleusercontent. com/search?q=cache:WmHtVpNIOkYJ:www.heartasso ciation.eu/it/cosa-e-il-ragionamento-clinico-e-perche-eimportante $/+\& \mathrm{~cd}=2 \& \mathrm{hl}=\mathrm{it} \& \mathrm{ct}=\mathrm{clnk} \& \mathrm{gl}=\mathrm{it}$

5. ten Cate O, Durning SJ. (2018) Understanding Clinical Reasoning from Multiple Perspectives: A Conceptual and Theoretical Overview. In: ten Cate O, Custers E, Durning S. (eds) Principles and Practice of Case-based Clinical Reasoning Education. Innovation and Change in Professional Education, vol 15. Springer, Cham, 2017.

6. Patel V, Arocha J, Zhang J. Thinking and reasoning in medicine, in: Holyoak K, Morrison R (Eds.). The Cambridge handbook of thinking and reasoning, Cambridge: Cambridge University PressPatel et al. 2005, pp. 727-750.

7. Frixione M. Come ragioniamo, Laterza, 2007.

8. Yazdani S, Hosseinzadeh M, Hosseini F. Models of clinical reasoning with a focus on general practice: A critical review, J Adv Med Educ Prof 2017; 5/4: 177-184.

9. Federspil G, Vettor R. La 'evidence-based medicine': una riflessione critica sul concetto di evidenza in medicina, ItalHeart J Suppl 2001; 2/6: 614-623.

10. Bruttomesso G. Il gap tra teoria e pratica in medicina generale, M.D. MedicinaeDoctor - Anno XVII numero 3 - 3 febbraio 2010: 10-11.

11. Nardi R. Intervento a favore di intuito e senso clinico alla base della gestione globale del paziente a rischio cardiovascolare, in: 'Alla ricerca del senso clinico smarrito: come sopravvivere tra interpretazioni statistiche isolate e convinzioni basate sull'esperienza', presentazione, Roma, The WestinExcelsior, 10-11 Marzo 2006.

12. Saarni SI, Gylling HA. Evidence based medicine guidelines: a solution to rationing or politics disguised as science?, J Med Ethics 2004, 30/2: 171-175.

13. Porzsolt F, Ohletz A, Thim A et al. Evidence-based decision making - the 6-step approach, ACP J Club 2003, 139: A11. doi:10.7326/ACPJC-2003-139-3-A11

14. Nardi R. Metodologia nell'approccio della valutazione delle competenze in Medicina Interna: la dimensione clinica, Metodologia nell'approccio della valutazione delle competenze in medicina interna, Roma, Ministero della salute, 16 gennaio 2018. Strumenti per la valutazione delle competenze professionali.

15. Bloom BS, Engelhart M, Furst EJ, Hill WH, Krathwohl DR, Taxonomy of educational objectives: The classification of educational goals. Handbook I: Cognitive domain. New York: David McKay Company, 1956.

16. Anderson LW, Krathwohl D. A Taxonomy for Learning, Teaching and Assessing: a Revision of Bloom's Taxonomy of Educational Objectives. Longman, New York, 2001; Churches A, Bloom's Taxonomy Blooms Digitally, 2008; http://www.techlearning.com/show Article.php?articleID $=196605124$

17. Schuwirth L. Is assessment of clinical reasoning still the Holy Grail? Med Ed 2009; 43: 298-299.

18. Pendleton H, \& Schultz-Krohn W. (2011) Types Of Clinical Reasoning . Pedretti's occupation therapy for physical dysfunction (7th ed.). St. Louis: Mosby.

19. Bowen JL. Educational Strategies to Promote Clinical Diagnostic Reasoning, Cox M. Irby DM Editors, N Engl J Med 2006; 355:2217-2225.

20. Murtagh J. Diagnostic modelling in General Practice, Aust Med Stud J. 2011; 2/1: 46-47.

21. Shadbolt N, Roberts C. Assessing Clinical Reasoning. The Clinical Judgement Test (CJT), https://www.york .ac.uk/media/healthsciences/images/research/mharg/DR EAMS2_Shadbolt_Roberts.pdf).

22. Stornello M. Value Based Medicine ed EvidenceBased Medicine, in Corso di perfezionamento congiunto, ' $\mathrm{La}$ gestione del paziente complesso: appropriatezza e competence' - I edizione Roma, settembre 2014 gennaio 2015.

23. Costa A. Habits of mind, http://www. habitsofmindinstitute.org/wp-content/uploads/2015/08/ Habits-of-Mind-w-icons-and-eduplanet.pdf

24. Bellavite P. La Complessità in Medicina. Fondamenti di un approccio sistemico-dinamico alla salute, alla patologia e alle terapie integrate, Tecniche Nuove Ed, Milano, 2009.

25. Sala V. La Medicina Narrativa: un nuovo approccio alla comunicazione medico-paziente, Corso di Laurea Interfacoltà in Comunicazione Interculturale e Multimediale, https://slideplayer.it/slide/534933/).

26. Tummino V. La comunicazione medico-paziente, Relazione presentata al Corso di alta formazione sul ragionamento clinico in era digitale, Bologna, Collegio San Tommaso, 16-20 settembre.

27. Puccio GJ, Murdock MC, Mance M. Current developments in creative problem solving for organizations: a focus on thinking skills and styles, Korean Journal of Thinking \& Problem Solving 2005, 15/2: 43-76.

28. Haynes RB. Forming research questions. J ClinEpidemiol. 2006; 59: 881-886.

29. Haynes RB, Sackett DL, Guyatt GH, et al. Clinical epidemiology: how to do clinical practice research. 3rd ed. New York (NY): Lippincott Williams and Wilkins; 2007.

30. Lazzari G, Salvini L, Patella S et al. La strutturazione del quesito clinico per reperire le prove di efficacia. Una revisione della letteratura, L'infermiere, 2015;52:5:e90e110, http://www.fnopi.it/ecm/rivista-linfermiere/rivista -linfermiere-page-30-articolo-346.htm

31. Oyinlola JO, Campbell J, Kousoulis AA. Is real world 
evidence influencing practice? A systematic review of CPRD research in NICE guidances. BMC Health Services Research 2016; 16: 299.

32. De Fiore L, Addis A. Real-world evidence, RecentiProg Med 2017; 108: 1-3). Anche la FDA è impegnata in un programma di approfondimento su questo tema (Framework for FDA's Real-World Evidence Program, https://www.fda.gov/downloads/ScienceResearch/Speci alTopics/RealWorldEvidence/UCM627769.pdf

33. Rothenbacher D, Capkun G, Uenal H et al. New opportunities of real-world data from clinical routine settings in life-cycle management of drugs: example of an integrative approach in multiple sclerosis, Current Medical Research and Opinion 2015, 31: 5: 953-965.

34. Perry CA. Knowledge bases in medicine: a review, Bull Med LibrAssoc 1990, 78/3: 271-280.

35. Bertolini G. Da una medicina basata sulle evidenze a una medicina basata sulle conoscenze, Recenti ProgMed 2014; 105: 7-8.

36. Porter ME. What is value in health care? N Engl J Med, 2010; 23; 363/26: 2477-2481.

37. Gray JAM. How to get better value healthcare, Oxford: Off ox Press, 2007.

38. Greenhalgh T, Howick J, Maskrey N. For the Evidence Based Medicine Renaissance Group, Evidence based medicine: a movement in crisis?, BMJ2014; 348: g3725 doi: $10.1136 /$ bmj.g3725

39. Sackett DL, Rosenberg WM, Gray JA, Haynes RB, Richardson WS. Evidence based medicine: what it is and what it isn't, BMJ 1996; 312/7023: 71-72).

40. Annovi G. Per una EBM fondata sui valori, Recenti ProgMed2016; SupplForward2; S25-S26.

41. Stephenson T, Maughan D, Ansell J. Protecting resources, promoting value: a doctor's guide to cutting waste in clinical care, Academy of Royal Medical College, November 2014, https://www.aomrc.org.uk/ wp-content/uploads/2016/05/Protecting_Resources_ Promoting_Value_1114.pdf

42. Berti F. Value based Medicine and Evidencebased Medicine,in: Corso di perfezionamento congiunto. La gestione del paziente complesso appropriatezza e competence, https://www.unicampus.it/documents/sfpl/ UCBM_Corso_di_Perfezionamento_La_gestione_del_pa ziente_complesso.pdf

43. Topol E. Deep Medicine: How Artificial Intelligence Can Make Healthcare Human Again, Basic Books; 1 edition (March 12, 2019).

44. Bell D et al. Dynamic Clinical Algorithms: Digital Technology Can Transform Health Care DecisionMaking Am. J. Trop. Med. Hyg., 98(1), 2018: 9-14.

45. Mushlin SB, Greene HL. Making in Medicine: An Algorithmic Approach, $3^{\text {rd }}$ Edition. Elsevier, 2010.

46. Ohno-Machado L. Clinical decision support: informatics interventions for better patient care, Journal of the American Medical Informatics Association, 25(5), 2018, 457, doi: 10.1093/jamia/ocy031

47. House of Commons Science and Technology Committee, Algorithms in decision-making Fourth Report of Session 2017-19, Report, together with formal minutes relating to the report Ordered by the House of Commons to be printed 15 May 2018-HC 351, published on 23 May 2018 by authority of the House of Commons.
48. Scantamburlo T, Charlesworth A, Cristianini N. Machine decisions and human consequences, A draft of a chapter that has been accepted for publication by Oxford University Press in the forthcoming book 'Algorithmic Regulation' edited by K. Yeung and M. Lodge due for publication in 2019.

49. Kwan N. The Hidden Dangers in Algorithmic Decision Making, https://towardsdatascience.com/the-hiddendangers-in-algorithmic-decision-making-27722d716a49

50. Editorial, Clinical decision making: more than just an algorithm, www.thelancet.com/oncology Vol 18 December 2017: 1553.

51. Di Francesco G. Unità capitalizzabili e crediti formativi. Metodologie e strumenti di lavoro, Milano, Franco Angeli, 1997.

52. Gensini GF, Conti AA. Il quesito clinico, RIMeL / IJLaM 2005; 1/Suppl: 24-27; Launer J, Guidelines and mindlines, PostgradMed J 2015; 91: 663-664.

53. Cartabellotta A. Linee guida, percorsi, processi, procedure, protocolli. Il caos regna sovrano: è tempo di mettere ordine!, GIMBE News, 2008 1: 4-5.

54. Cartabellotta A. Facciamo luce sull'EBM. Sanità \& Management 2003, Conoscere 1'EBM. Ambito di applicazione, https://www.gimbe.org/eb/ambiti.html

55. Liberati A. Linee-guida ed 'evidence-based medicine': l'importanza di distinguerne i ruoli, EpidemiolPrev 1996; 20: 277-278.

56. Carrigan T. Evidence-based and guideline-based medicine, J AccidEmerg Med 2000; 17: 154-155.

57. Mutasingwa DR, Ge H, Upshur REG. How applicable are clinical practice guidelines to elderly patients with comorbidities?, Can Fam Physician 2011;57: e253-262.

58. Cartabellotta A. Medicina basata sulle evidenze o sulle linee guida?, Ricerca E Sanità 2000, /2: 41-43).

59. Occhini L, Pulerà A, Felici M. L'illness del paziente: una EvidenceBased Medicine nella medicina della complessità,Quaderni - Italian Journal of Medicine 2017, 5/1: 39-45.

60. Lenti S, Felici M, Campanini M, Fontanella A, Nardi R, Gussoni G. La comunicazione con il paziente e tra professionisti nella gestione del paziente complesso in Medicina Interna, Italian Journal of Medicine 2017, 5/1: 1-81. https://doi.org/10.4081/itjm.q.2017

61. Bacon F. MeditationesSacrae, 1597.

62. Adamo M. Comunicazione personale, 1 giugno 2019.

63. Spence D. The lives of others, BMJ 2014; 348: g2209.

64. Editorial, Uncertainly in medicine, The Lancet 2010, 375/9727: 1666.

65. Kleinman A. The art of medicine. Catastrophe and caregiving: the failure of medicine as an art. Lancet 2008; 371: 22-23.

66. Ofri D. The art of medicine. The muse on the medical wards. Lancet 2008; 371: 110-111.

67. Lawrence C. The art of medicine. Hippocrates, society, and utopia. Lancet 2008, 371: 198-199.

68. Platone, Sofista, 235d-236c, 265a e segg.

69. Stoichita VI, Breve storia dell'ombra. Dalle origini della pittura alla Pop Art, Il Saggiatore, 2008.

70. Aristotele, Opere, Metafisica, Laterza Bari, 1988.

71. Fortuna F, Tiberio A. Il mondo dell'empatia. Campi di applicazione, Franco Angeli 1999.

72. Cosmacini G. La medicina non è una scienza: breve 
storia delle sue scienze di base, R. Cortina Editore, 2008.

73. Wellbery C. The art of medicine The value of medical uncertainty?, The Lancet 2010, 375 May 15: 16861687.
74. The Institute of Complexity Medicine, https://www. complexitymedicine.org/intro

75. Montgomery K. How Doctors Think. Clinical Judgment and the Practice of Medicine, Oxford University Press, 10 November 2005. 


\title{
Competenza e metodologia in Medicina Interna: quali strumenti di valutazione delle capacità di ragionamento clinico? Seconda Parte
}

\author{
Flavio Tangianu, ${ }^{1}$ Antonino Mazzone, ${ }^{2}$ Giuliano Pinna, ${ }^{3}$ Giuseppe Chesi, ${ }^{4}$ Paola Gnerre, ${ }^{5}$ Franco Berti, ${ }^{6}$ \\ Michele Stornello, ${ }^{7}$ Carlo Nozzoli, ${ }^{8}$ Salvatore Corrao, ${ }^{9}$ Mauro Silingardi, ${ }^{10}$ Giovanni Mathieu, ${ }^{11}$ Andrea Fontanella, ${ }^{12}$ \\ Daniele Borioni, ${ }^{13}$ Roberto Nardi ${ }^{13}$
}

${ }^{1}$ Medicina Interna, Dipartimento di Medicina, Università di Insubria (VA); ${ }^{2}$ Medicina Interna, Legnano (MI); ${ }^{3}$ Medicina Interna, Asti; ${ }^{4}$ Dipartimento di Medicina Interna, Ospedale Magati di Scandiano, AUSL di Reggio Emilia; ${ }^{5}$ Medicina Interna 2, Ospedale San Paolo, Savona; ${ }^{6}$ Medicina Interna, Roma; ${ }^{7}$ Medicina Interna, Siracusa; ${ }^{8}$ Medicina Interna, Azienda Ospedaliero-Universitaria Careggi, Firenze; ${ }^{9}$ Dipartimento di Medicina Clinica, ARNAS Civico Di Cristina Benfratelli, Palermo; PROMISE, Università di Palermo; ${ }^{10}$ Medicina Interna, Ospedale Maggiore, Bologna; ${ }^{11}$ Medicina Interna, ASL Torino 3, Piemonte; ${ }^{12}$ Medicina Interna, Ospedale Madonna del Buon Consiglio Fatebenefratelli, Napoli; ${ }^{13}$ Medicina Interna, Bologna, Italia

\section{Introduzione}

La valutazione delle capacità di ragionamento clinico costituisce un problema complesso. Un singolo strumento non è mai sufficiente - di per sé - per valutare il ragionamento diagnostico. Viceversa, un programma di valutazione che impieghi più strategie integrate può fornire un processo più efficace per determinare la competenza del medico nel ragionamento diagnostico. Restano insolute alcune domande su come i processi di ragionamento clinico possano essere misurati in maniera più accurata. Questo è un ulteriore tema per la ricerca futura, su cui le Società Scientifiche potranno impegnarsi.

\section{Seconda Parte: Modalità di Valutazione delle Capacità di Ragionamento Clinico}

La valutazione delle competenze del medico è un procedimento assai complesso. Per tale obiettivo un unico strumento non è di per sé sufficiente, in relazione alla molteplicità dei problemi e degli obiettivi in causa $^{1-3}$ (Tabella 1; Figura 1)., ${ }^{4,5}$

Corrispondente: Roberto Nardi, Medicina Interna, Bologna, Italia.

E-mail: nardidoc48@gmail.com

Articolo pubblicato secondo la Creative Commons Attribution NonCommercial 4.0 License (CC BY-NC 4.0).

${ }^{\circ}$ Copyright: the Author(s), 2020

Licensee PAGEPress, Italy

QUADERNI - Italian Journal of Medicine 2020; 8(1):105-124
In particolare, la valutazione della capacità di ragionamento clinico dell'internista, attinente a livelli di pensiero epicritico, dovrebbe essere desunta dalle soluzioni proposte a fronte di problemi complessi, dal loro razionale e dai comportamenti messi in atto per addivenire alla diagnosi finale. Essa infatti si riferisce ad una competenza intangibile, non direttamente misurabile, che andrebbe correlata alla conoscenza e all'esperienza del singolo professionista, nonché al contesto operativo. Tale valutazione dovrebbe essere diversa se riferita ad un medico 'novizio' o ad uno esperto. ${ }^{6}$ Per poter giudicare le competenze dell'internista non si può prescindere da elementi integrati di un programma complessivo di formazione, con definizione degli obiettivi formativi e della performance richiesta. La valutazione della clinical competence dovrebbe infatti basarsi su una metodologia composita, impiegando strumenti differenziati, allo scopo di verificare: i) le conoscenze di base e le abilità cognitive avanzate; ii) la capacità di ragionamento critico; iii) l'accuratezza diagnostica rispetto ai molteplici problemi posti dal paziente; iv) la capacità di decidere anche in situazioni di complessità e/o di incertezza; v) la capacità di fare e di comunicare ciò che stiamo facendo; vi) la capacita di «rendere conto» ('accountability') delle scelte fatte, in base ad una specifica consapevolezza ('awareness') di quanto attuato da parte di ciascun singolo professionista nel contesto organizzativo assistenziale. ${ }^{7}$ La capacità d'identificare una corretta diagnosi finale, indipendentemente dal numero di tentativi intrapresi, costituisce un elemento importante nella valutazione del ragionamento clinico. Per la formazione, la valutazione e la ricerca del ragionamento clinico è consigliabile concentrarsi maggiormente sui dettagli del processo utilizzati per raggiungere una diagnosi corretta, piuttosto che sul 
Tabella 1. Alcuni strumenti di valutazione della competenza in funzione degli obiettivi. (Modificata $\mathrm{da}^{4}$ ).

\begin{tabular}{ll}
\hline Strumento/metodo & Capacità richieste e obiettivi \\
\hline Portfolio & $\begin{array}{l}\text { Esperienze precedenti, curriculum vitae personale, professionalism, certificazione delle esperienze } \\
\text { pregresse e delle procedure effettuate }\end{array}$ \\
\hline Domande a scelta multipla & Cognitive (saper decidere e scegliere, in base alle conoscenze) \\
\hline Presentazione orale di un caso clinico & Comunicative/argomentative, capacità di elaborare un pensiero critico/epicritico \\
\hline Incontro con paziente reale & $\begin{array}{l}\text { Comunicative, empatico-cognitive, semeiologiche, capacità di selezione delle priorità, di alto livello } \\
\text { cognitivo-decisionale }\end{array}$ \\
\hline Esame orale & Di eloquio, comunicazione, argomentazione, problem solving \\
\hline Key features problems & Di alto livello cognitivo-decisionale \\
\hline Feedback & Comportamentali pregresse (da cartelle cliniche, manuali e documenti di accreditamento) \\
\hline Prova pratica su manichino & Tecnico-manuali e operative simulative \\
\hline Prova pratica fra pari & $\begin{array}{l}\text { Congruenza con standard di riferimento validati (osservazione, confronto diretto tra colleghi, video- } \\
\text { registrazione, confronto } v \text { standard predefiniti) }\end{array}$ \\
\hline Relazione clinica scritta & $\begin{array}{l}\text { Comunicative, relazionali (capacita di formulare un elaborato scritto in maniera formalmente } \\
\text { corretta), argomentativi (capacità di elaborare un pensiero critico e di discuterlo in maniera congrua) }\end{array}$ \\
\hline Revisione critica d ella letteratura & $\begin{array}{l}\text { Capacità di argomentare e documentare con appropriati strumenti metodologici, statistici, } \\
\text { clinicamente utili }\end{array}$ \\
\hline Script concordance test & Caratteristiche cognitive, di alto livello \\
\hline Situational judgement test & Caratteristiche di professionalism ed etica clinica \\
\hline Stazioni OSCE & \begin{tabular}{l} 
Caratteristiche interpretative, capacità decisionali, comunicative, tecnico-manuali \\
\hline
\end{tabular} \\
\hline
\end{tabular}

\section{MODALITA' DI VALUTAZIONE DELLA COMPETENZA PROFESSIONALE}

\section{Test di valutazione scritti}

(valutazione delle conoscenze)

\section{Valutazione sul campo da parte dei supervisori/board scientifico)}

(anche mediante strumenti informatici)
- Quiz a risposta multipla (vero-falso)

- La migliore di cinque quiz a risposta multipla

- Accoppiamento su opzioni multiple

- Prove su temi scritti:

?. la composizione (redazione, dissertazione), consente di valutare le conoscenze, ma anche la capacità di analisi, di sintesi, l'espressione scritta

- Risposte brevi su domande aperte

- Key feature problems

- Autovalutazione mediante check list o questionari semiquantitativi
Prove cognitive e relazionali:

risoluzione e discussione di casi clinici,

revisione di ricerche, revisione di incidenti critici,

capacità didattica

per esplorare il ragionamento clinico su un paziente

per valutare le capacità di comunicazione e d' interazione in un gruppo

professionale

Prove gestuali: osservazione diretta nell' esecuzione di procedure:

per valutare la conoscenza, l' abilità pratica e procedurale e le attitudini del medico nell' interazione con il paziente.

Prove relazionali: gioco di ruolo (role play),

per valutare la gestione della tensione nervosa, la reattività, la capacità d'adattamento.

Osservazione diretta "bed-side" o con simulazione a distanza/ video o con controllo del processo di assistenza e cura mediante I' impiego di:

Dgriglie di valutazione

Dliste di controllo

口"blueprint" assessment

$\square$ macro-micro-simulazione-skill trainer

Figura 1. Modalità di valutazione della competenza professionale. ${ }^{5}$ 
primo tentativo di ipotesi diagnostica. ${ }^{8}$ Come è possibile misurare e valutare il ragionamento clinico che avviene nella mente di ogni singolo medico? In molti casi se ne possono dedurre i passaggi fondamentali, ma bisogna che il ragionamento venga esplicitato dal singolo professionista per poterlo valutare. Un programma di valutazione che impiega più strategie integrate può fornire il processo più robusto per determinare la competenza del medico nel ragionamento diagnostico. L'ABIM (American Board of Internal Medicine) ha definito alcune modalità di valutazione delle capacità di ragionamento clinico ('clinical Judgment/reasoning), in particolare in merito alle modalità di perseguire la diagnosi in maniera appropriata, considerando rischi e benefici del percorso prescritto: i) PCPS (Patients care and procedural skills): attinenti alla capacità di garantire un'assistenza sanitaria adeguata, con procedure sicure ed efficaci, finalizzata alla promozione della salute; ii) MK (Medical Knowledge): la conoscenza dell'epidemiologia e delle scienze bio-mediche, cliniche e sociali deve essere applicata all'assistenza realmente praticata; iii) PBLI (Practice based learning and improvement): gli esaminandi devono dimostrare di saper perseguire nuove evidenze e di autovalutazione delle proprie conoscenze in un processo continuo, per migliorare l'assistenza fornita ai pazienti; iv) SBP (System Based Practice): gli esaminandi devono saper evidenziare responsabilmente il senso di appartenenza al 'sistema' sanitario locale e la loro capacità di coinvolgere efficacemente ed in maniera appropriata le altre risorse disponibili, per garantire un'assistenza ottimale. ${ }^{9} \mathrm{Di}$ seguito sono considerati alcuni dei possibili strumenti oggi disponibili per tale non semplice compito. ${ }^{10}$

\section{A - Valutare il ragionamento diagnostico al di fuori del contesto clinico}

\section{A.1 - Esami orali}

Diversi studi suggeriscono che gli esami orali possono essere strumenti di valutazione validi. L'American Board of Emergency Medicine utilizza una prova orale consistente in scenari clinici per valutare il ragionamento diagnostico. Gli esperti clinici forniscono simultaneamente informazioni sequenziali sullo scenario e valutano il partecipante utilizzando un modello strutturato su azioni e punti chiave, in un'interazione diretta e reciprocamente 'comunicativa' con l'esaminando. ${ }^{11,12}$

\section{A.2 - Domande a scelta multipla (multiple-choice questions-MCQs)}

Le domande a scelta multipla (multiple-choice questions-MCQs) sono un metodo consolidato e affidabile per valutare la conoscenza. La costruzione di test efficaci con MCQs deve basarsi su una scrupolosa progettazione, revisione e validazione, sulla base di esperienze e conoscenze molto approfondite e con molto tempo a disposizione. ${ }^{13} \mathrm{Un}$ evidente limite degli MCQs ai fini della valutazione del ragionamento diagnostico è che l'elenco delle scelte predefinite su cui esprimersi può suggerire risposte relative alle ipotesi diagnostiche in causa. Nella pratica clinica un passaggio cruciale nel processo decisionale è dato dal fatto che la generazione delle ipotesi e le opzioni diagnosticodifferenziali devono scaturire de novo da parte del professionista: tale assioma può non essere rispettato nel formato tradizionale dei quiz a risposta multipla.

\section{A.3 - I problemi della caratteristica principale (key feature problems-KFP)}

Partono da elementi narrativi, alternati a domande, rappresentando una specie di caso clinico simulato scritto. Si basano sul concetto di passaggi critici o 'caratteristiche chiave' nel processo decisionale e rappresentano un'evoluzione rispetto al formato basato sulla gestione dei problemi. ${ }^{14,15}$ Viene proposto un scenario clinico seguito da una serie di domande a risposta aperta sui passaggi essenziali per la risoluzione del caso. Il candidato è chiamato a pensare all'identificazione dei problemi principali, alle strategie diagnostiche e alle decisioni finali. Tale metodo è risultato particolarmente affidabile per la valutazione della capacità di ragionamento clinico, ${ }^{16}$ anche per scegliere le indagini più appropriate, essenziali ed economicamente più vantaggiose per un'adeguata gestione a breve e lungo termine dei problemi del paziente ${ }^{17}$ (Appendice 1).

\section{A.4 - Script concordance test (SCT)}

Una specifica presentazione del paziente può richiamare al medico un precedente caso clinico, che si integra con le sue precedenti conoscenze di merito su vari aspetti correlati (implicazioni patofisiologiche, trattamento, complicanze, eccetera). In effetti, i clinici esperti sviluppano mentalmente, nel proprio vissuto professionale, 'script di malattia', come un repertorio personale di storie di pazienti che collegano esperienze cliniche precedenti con conoscenze mediche e li memorizzano come ricordi accessibili in grado di guidare il ragionamento diagnostico per nuovi casi. ${ }^{18}$ Gli script concordance test (SCT) sono utilizzati, a partire dalla descrizione di un caso, per valutare il ragionamento clinico in situazioni ambigue o incerte: permettono di considerare situazioni reali, generalmente non adeguatamente misurabili con altri test attualmente disponibili. ${ }^{19}$ Costituiscono strumenti validi per la 
loro affidabilità, anche nel discriminare le capacità tra diversi livelli di esperienza. ${ }^{20}$ Gli SCT possono essere strutturati anche per sondare le conoscenze sull'uso di test diagnostici o degli interventi terapeutici. Si basano sulla teoria della psicologia cognitiva e sulla teoria dell'illness script (rappresentazione della malattia: la storia clinica viene confrontata con altri casi già affrontati nel corso degli studi e dell'esperienza professionale), con tre caratteristiche chiave di progettazione: i) esaminare casi e situazioni cliniche non immediatamente chiare e definite, scegliendo tra diverse opzioni realistiche rispetto al caso; ii) il formato delle risposte dovrebbe riflettere il modo in cui le informazioni vengono elaborate in una logica di problem-solving complesso; iii) il punteggio tiene conto della variabilità delle risposte date da anche clinici esperti in situazioni cliniche complesse analoghe. A differenza degli MCQs, in cui esiste sempre una risposta più corretta, questo metodo confronta le risposte degli esaminandi con l'intervallo di risposte generato da un pannello di riferimento di esperti. Il punteggio si basa sul grado di concordanza con il pannello di riferimento $^{21}$ (Appendice 2).

\section{A.5 - OSCE: \\ Objective Structured Clinical Examination}

L'Objective Structured Clinical Examination (OSCE, esame clinico strutturato e obiettivo) è un metodo di approccio per la valutazione obiettiva pianificata e strutturata - della competenza clinica nelle sue diverse componenti. ${ }^{22}$ Possono essere valutati diversi aspetti, quali: anamnesi, esame obiettivo, abilità professionalizzanti mediche e chirurgiche, diagnostica di laboratorio, diagnostica strumentale, abilità comunicative, performance psicorelazionali e capacità di sintesi. Nell'OSCE il professionista passa consecutivamente attraverso una serie di stazioni (Figura 2). In ciascuna viene sottoposto alla valutazione di una particolare performance. Vi sono vari tipi di stazioni: i) cliniche: si valuta l'interazione tra professionista ed un paziente standardizzato; ii) pratiche: necessitano di attrezzature e di un esaminatore; iii) statiche: esami, radiografie, ecc. Il tempo a disposizione dell'esaminando è solitamente prestabilito e limitato, al massimo 10 minuti le prove più complesse, sulla base del fatto che nelle situazioni reali il tempo è sempre limitato. All'OSCE si sono successivamente aggiunti altri

\section{Cos' è l' OSCE \\ Objective Structured Clinical Examination}

- Più che un metodo di esame è un' intelaiatura che può incorporare diversi metodi di valutazione

- E' costituito da una serie di stazioni attorno alle quali ciascun professionista ruota

- Utilizza:

- criteri di valutazione predefiniti per ciascuna performance

- giudizi stabiliti mediante griglie di valutazione

- Rapporti tra esaminando ed esaminatore regolati e predeterminati

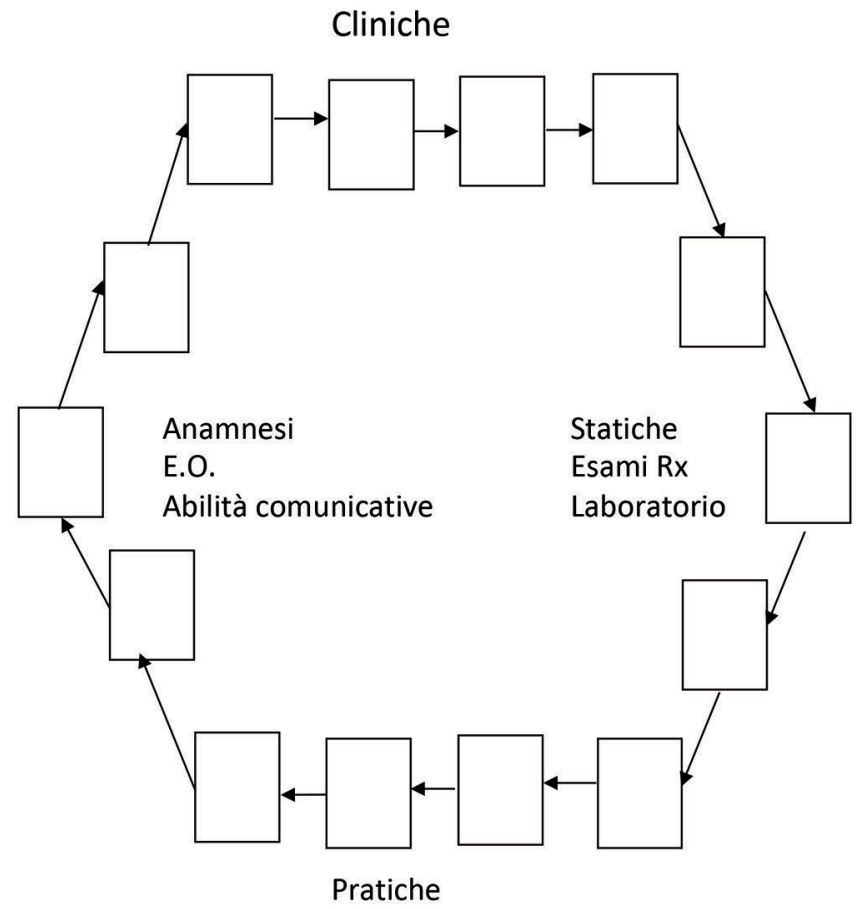

Figura 2. Cos'è l'OSCE. 
strumenti di valutazione, che di fatto ne costituiscono varianti: OSLER (Objective Structured Long Examination Record), OSPE (Objective Structured Pratical Examination), OSVE (Objective Structured Video Examination), OSTE (Objective Structured Teaching Evaluation), OSPRE (Objective Structured Performance Related Examination), OSSE (Objective Structured Selection Exam). ${ }^{23}$

\section{A.6 - Prove di valutazione in particolari situazioni (Situational judgement test-SJT)}

Le prove di valutazione in particolari situazioni fanno parte dell'OCSE. ${ }^{24}$ Sono rivolte a professionisti sia junior che senior, allo scopo di valutarne le capacità 'soft', quali abilità cognitive, intelligenza sociale ed emotiva, pensiero analitico, processo decisionale finalizzato alla soluzione dei problemi, pianificazione ed organizzazione, elasticità, flessibilità, capacità di adattamento al contesto organizzativo. Le situazioni presentate possono riguardare difficoltà con i pazienti e i loro famigliari, possibili condizioni di stress, un precario clima organizzativo, conflitti tra professionisti, eccetera. Questo metodo presuppone alcune opzioni riguardo alle modalità di risposta: i) most and least effective (più efficace e meno efficace); ii) rated responses (punteggio per ogni risposta). Un possibile limite di questo metodo è dato dal fatto che in situazioni difficili riconoscere correttamente le risposte come inappropriate (cosa non si dovrebbe fare), pur avendo una maggiore validità rispetto alle risposte giudicate come appropriate (ciò che si dovrebbe fare) non è semplice: nel giudizio complessivo sembra comunque meglio valutare le opzioni orientate a riconoscere ciò che non si dovrebbe fare. ${ }^{25}$ Un esempio di questa tipologia di test è riportato in Appendice 3.

\section{A.8 - Prova basata sulle caratteristiche principali (Key-feature questions-KFQs)}

Il formato di un test basato sulle caratteristiche principali (KFQs) si basa sul concetto fondante di un'importante nozione clinica sulla 'specificità del caso'. I casi sono progettati per valutare in modo specifico le capacità decisionali complesse, con richiamo alla conoscenza effettiva, ma anche alla capacità di applicare la conoscenza alla risoluzione di un problema. Ad esempio non si dovrebbe solamente saper descrivere le caratteristiche del delirium tremens (un problema di conoscenza), ma si dovrebbero valutare le capacità degli esaminandi di riconoscere il delirium tremens 'in un paziente specifico' (che include il ragionamento clinico), per proporre appropriate misure terapeutiche (ovvero la decisione clinica). Tale scomposizione del processo si concentra su diverse abilità e non solamente sul semplice riconoscimento ed elencazione delle caratteristiche cardinali del delirium tremens ${ }^{26,27}$ (Appendice 4).

\section{A.9 - Pazienti virtuali e casi simulati}

La simulazione è una tecnica impiegata per sostituire o amplificare casi clinici vissuti attraverso esperienze virtuali, guidate in maniera interattiva, in grado di riprodurre aspetti sostanziali del mondo reale, rappresentati anche in maniera non lineare. ${ }^{28}$ La realtà simulata che riproduce scenari clinici realistici al computer è uno strumento moderno ed in evoluzione costante che permette l'apprendimento e la verifica di competenze cliniche. Gli ambiti di simulazione in medicina - con relative valutazioni delle performances dei professionisti - possono essere diversi: ${ }^{29}$ i) verbale (giochi di ruolo - role playing); ii) con pazienti standardizzati (attori); iii) per specifici compiti operativi (PTT - 'part-task trainers'), mediante strumenti fisici o virtuali utilizzati per un particolare obiettivo formativo dell'intero processo; iv) paziente 'computerizzato' (visualizzazione a schermo del computer o realtà virtuale sempre tramite schermo video); v) paziente elettronico (repliche di pazienti e scenari clinici in ambito realistico, manichini, video, realtà virtuale totale). ${ }^{30}$ L'impiego della simulazione in Medicina Interna è particolarmente utile per valutare la conoscenza clinica ed il processo decisionale, oltre che per identificare le esigenze di apprendimento. ${ }^{31}$ La simulazione presuppone alla fine del processo una discussione (debriefing), con l'intervento di un facilitatore esperto (tutor) per l'apprendimento mediante feedback e riflessione collegiale su quanto svolto. L'educazione basata sulla simulazione può costituire un potenziale strumento di valutazione del ragionamento diagnostico, in base al 'ciclo di apprendimento' di Kolb. ${ }^{32}$ Questo presuppone un processo imperniato in 4 fasi: i professionisti imparano attraverso l'esperienza concreta (fase 1), la riflessione sull'esperienza (fase 2), la concettualizzazione delle loro osservazioni riflessive in modelli più astratti (fase 3) e la sperimentazione di questi nuovi principi e conclusioni, allo scopo di definire le successive decisioni e azioni che portano a nuove esperienze concrete (fase 4). La fase 2 e 3 sono componenti prevalenti del debriefing, che permette agli esaminandi e al tutor di discutere i flussi di ragionamento clinico esperiti. ${ }^{33}$ I pazienti virtuali, pur essendo un metodo di apprendimento ottimizzato dalla tecnologia per insegnare il ragionamento clinico, non sfruttano appieno il loro potenziale, in quanto prevalentemente focalizzati ai risultati piuttosto che all'esplorazione della correttezza del processo di ragionamento clinico. Per 
questo si stanno sviluppando nuovi strumenti tecnologici finalizzati a valutare specificamente le modalità di clinical reasoning in questo contesto. ${ }^{34}$ Secondo una recente revisione sistematica, la valutazione basate sulla simulazione può utilizzata in modo efficace, ma non ancora come strumento autonomo impiegato da solo. ${ }^{35}$ L'educazione medica basata sulla simulazione può rappresentare uno strumento utile e realistico, con ammissibilità di errore in un contesto controllato. Proprio attraverso la lente dei possibili errori diagnostico-decisionali, è stato evidenziato che quasi un quarto degli esaminati mediante simulazione ha assunto decisioni potenzialmente errate e dannose. ${ }^{36-38}$ È evidente che il contatto diretto con il paziente è quello più efficace per educare e valutare i migliori medici clinici. ${ }^{39}$

\section{A.10 - Lo strumento IDEA}

Lo strumento IDEA (Interpretive summary, Differential diagnosis, Explanation of reasoning, and Alternatives), basato sulla capacità di porre una credibile diagnosi differenziale, in base alle capacità interpretative dei dati, di spiegazione del ragionamento effettuato e di valutazione delle alternative proposte (IDEA) è stato sviluppato per valutare le capacità di ragionamento diagnostico e decisionali degli esaminandi con esame scritto. La sua affidabilità, pur se non assoluta, fa di questo un ulteriore strumento rispetto a modalità di giudizio di tipo 'sommario' ${ }^{40}$ (Appendice 5).

\section{A.11 - Simulazione basata sul lavoro di squadra (team-based simulation)}

Il 'senso di appartenenza' dei professionisti è fondamentale per perseguire i progetti aziendali nell'interesse dei pazienti. Il 'lavorare in squadra', in un contesto organizzativo complesso come quello ospedaliero, è d'obbligo. Anche se le esperienze di team based simulation sono più spesso riferite al trauma team, tale metodologia può essere estesa alla simulazione di situazioni critiche, proprio per la valutazione delle 'non technical skills', quali le capacità dei singoli professionisti di lavorare ed interagire nell'équipe multi-professionale e/o multispecialistica, aderendo ai ruoli e ai protocolli assegnati, con l'obiettivo ultimo di ridurre gli errori e il rischio di mortalità. Attualmente con l'impiego di 'simulatori ad alta fedeltà', ovvero di manichini o di supporti informatici molto evoluti controllati da software complessi, è possibile riprodurre, in modo molto realistico, l'ambiente di lavoro e il 'clima' che si crea in situazioni di crisi o di 'stress' in un gruppo professionale. Sono però necessari ulteriori studi per definirne l'utilizzo in terapia intensiva. ${ }^{41}$

\section{B - Valutare direttamente il ragionamento diagnostico nel contesto clinico}

Nel contesto clinico sono diversi gli strumenti per un'osservazione diretta degli esaminandi. L'osservazione diretta è molto utile per valutare competenze mirate. Il senior osserva direttamente la visita al paziente nel suo complesso, sia dal punto di vista sia clinico che relazionale. L'osservazione diretta con mini-CEX e la discussione basata sui casi sono tra gli strumenti importanti più comunemente utilizzati per la valutazioni dei professionisti sul posto di lavoro ('work-based assessment approach'). ${ }^{42}$

\section{B.1 - Osservazione diretta (mini-clinical-evaluation exercise-MiniCex)}

Il 'mini-clinical-evaluation exercise' (mini-CEX) è stato sviluppato per l'osservazione diretta degli esaminandi, con un medico supervisore valutatore allo scopo di verificare, in un contesto clinico, le capacità d'interazione con il paziente, le modalità di raccolta dei dati anamnestici e di corretta esecuzione della visita medica. In definitiva il mini-Cex, introdotto originariamente dall'American Board of Internal Medicine, è un metodo di valutazione delle competenze essenziali. ${ }^{43,44}$ Le aree valutate riguardano anche la professionalità, le capacità di ragionamento e di giudizio clinico (ad esempio la capacità di richiedere/ effettuare in modo selettivo accertamenti diagnostici appropriati; di considera rischi e benefici del trattamento prescritto), le capacità di comunicazione, l'organizzazione/l'efficienza complessiva del processo diagnostico e terapeutico. Il Mini-CEX utilizza una scala di valutazione a nove punti: punteggi da 1 a 3 denotano un risultato non soddisfacente, da 4 a 6 soddisfacente, da 7 a 9 superiore. Una valutazione di 4, pur essendo classificata come soddisfacente, è definita come 'ai limiti' (borderline) e dovrebbe indurre l'esaminando a migliorare le proprie prestazioni con un adeguato programma di formazione (Appendice 6).

\section{B.2 - DOPS: Direct Observation of Procedural Skills (Directly Observed Procedural Skills)}

L'osservazione diretta delle competenze procedurali (Directly Observed Procedural Skills, DOPS) consiste nell'osservazione e nella valutazione delle procedure eseguite in un paziente reale. Le abilità tecniche o pratiche valutate sulla base del DOPS variano da quelle relativamente semplici e comuni (come il prelievo venoso) fino a quelle più complesse (ad esempio, colangiografia retrograda endoscopica). La valutazione viene effettuata da un medico senior esperto, che utilizza un elenco di item e compiti predefiniti, con una graduazione 
differenziata per livelli di competenza accertata (per esempio: sotto le aspettative: 1-2; borderline: 3; entro le aspettative: 4; al di sopra delle aspettative: $5-6)^{45}$ (Appendice 7).

\section{B.3 - VOPS: Video Observation of Procedural Skills}

Dopo aver ottenuto il consenso del paziente, l'esaminando registra in video la visita e le procedure effettuate. In seguito la video-registrazione viene revisionata dal senior (meglio se in un briefing collegiale aperto al board scientifico e agli altri esaminandi). Questo metodo offre l'importante vantaggio di poter interrompere la visione della registrazione nei punti ritenuti fondamentali per analizzare e discutere i problemi e/o le criticità rilevate. ${ }^{46}$

\section{B.4 - Il modello di ruolo (shadowing-role modelling)}

Il 'modello di ruolo' corrisponde ad una persona 'esperta', il cui comportamento è esempio di successo, tale da poter essere emulato da altri, in particolare da persone più giovani. La 'modellazione dei ruoli' serve a trasmettere 'le capacità intangibili, da alcuni chiamate l'arte della medicina'. ${ }^{47}$ Questo metodo consente di valutare fianco a fianco l'atteggiamento dell'esaminando nei confronti del paziente, l'approccio professionale, la capacità di gestione delle situazioni difficili e del trattamento di cura. Limiti di questa tecnica sono l'apprendimento passivo e l'affidarsi alla sola capacità critica di osservazione e riflessione da parte dell'esaminando su un unico elemento di riferimento. ${ }^{38}$

\section{B.5 - Feedback dei pazienti e dei colleghi (Multisource Feedback-MSF)}

Le competenze generali degli internisti possono essere valutate da colleghi medici, infermieri e pazienti. ${ }^{48}$ Il feedback multi-source (MSF) o 'feedback da più fonti' è uno strumento di riscontro diversificato per una valutazione interattiva tra persone che interagiscono tra loro sulle decisioni assunte. ${ }^{49}$ Tale feedback incrociato viene utilizzato per individuare $\mathrm{i}$ punti di forza e di debolezza, gli ambiti di sviluppo personale di ciascuno tramite relazioni dirette, confronti tra pari e contatti esterni. Negli Stati Uniti, il MSF è un componente dei programmi di manutenzione dei certificati (Maintenance of Certification-MOC) dell'American Board of Medical Specialties (ABMS) ed è un requisito per la valutazione dei residenti, su mandato del Consiglio di accreditamento del Graduate Medical Education. Più recentemente anche il Medical Board of Australia's Professional Performance Framework ha confermato il continuo feedback dei pazienti e dei colleghi come utile strumento di valutazione dello sviluppo professionale continuo. ${ }^{50}$
C - Valutare il ragionamento clinico post-hoc: analisi retrospettiva dei casi in base alla revisione tra pari, ai report e alla documentazione clinica disponibile

\section{C.1 - Audit clinico}

L" 'Audit è una analisi critica e sistematica della qualità delle cure mediche, che comprende procedure diagnostiche, terapeutiche ed assistenziali, nonché l'utilizzo di risorse e la valutazione degli esiti e della qualità di vita dei pazienti'. ${ }^{51} \mathrm{E}$ 'un' iniziativa condotta da professionisti che cerca di migliorare la qualità e gli esiti dell'assistenza, attraverso una revisione tra pari, strutturata, con la quale i professionisti esaminano la propria attività e $\mathrm{i}$ propri risultati a confronto con standard concordati e la modificano se necessario'. L'audit clinico fornisce quindi un metodo per riflettere in maniera sistematica sulla propria pratica professionale e per rivederla. In sintesi è la 'verifica della pratica corrente rispetto a criteri e standard noti'. ${ }^{52-56}$

\section{C.2 - La valutazione dei processi assistenziali: il blue print assessment}

La valutazione dei processi assistenziali costituisce il metodo che presenta i maggiori vantaggi ai fini della valutazione della competenza professionale. Essa infatti include la valutazione sia dei processi generali (approccio al malato, capacità decisionale, razionalità, conoscenza, saper fare, relazionalità e comunicazione con i malati, i parenti e il personale che opera nei reparti, capacità diagnostiche, prognostiche $\mathrm{e}$ terapeutiche, consulenze specialistiche, prevenzione, riabilitazione, rapporti con il territorio, ecc.), sia di processi specifici, orientati sulla singola situazione clinica e sul singolo paziente, valutato nella sua complessità, criticità e priorità di decisioni'. Tra gli strumenti impiegati nella valutazione della clinical competence in correlazione allo sviluppo dei processi e sotto-processi assistenziali deve essere considerato il cosiddetto 'blueprint assessment' (da blueprint: schema, progetto, piano, programma, struttura, piano d'azione, cianografia). In sostanza il blueprint definisce il risultato che si desidera ottenere con le azioni che è necessario porre in essere (come dire: ' $v a$ fatto esattamente cosi'), per realizzare il prodotto voluto in un processo di assistenza ottimizzato. ${ }^{57-60}$ L'ABIM propone un esame denominato 'Internal Medicine Certification Examination Blueprint', progettato per valutare negli internisti le conoscenze, il ragionamento diagnostico e le capacità di giudizio clinico e per di proporre appropriate decisioni diagnostiche e gestionali che hanno importanti conseguenze per i pazienti su problemi clinici sia comuni che rari. ${ }^{61}$ 


\section{C.3 - Metodi basati sulla revisione e discussione della cartella clinica: metodi CLAP, CR e CSR}

La cartella clinica può essere impiegata per valutare le capacità dei professionisti del 'clinical problem solving'. ${ }^{62}$ In un primo approccio, alcuni elementi di valutazione della qualità della cartella clinica (metodo 'CLAP': completezza, leggibilità, accuratezza, professionalità), ${ }^{63,64}$ riportati nella Tabella 2, possono essere utilizzati.

Le tecniche CR (Chart Review) e CSR (ChartStimulated Recall) si basano su una valutazione più approfondita dei dati riportati in cartella clinica, considerando la diligenza, la completezza e la pertinenza delle informazioni riportate nelle diverse fasi del processo diagnostico-terapeutico. Il candidato deve rispondere a domande predefinite, sulla base di una revisione critiche delle varie parti/fasi del processo deducibile dalla documentazione consultata (Tabella 3). ${ }^{65}$

L'impiego della cartella clinica informatizzata facilita di gran lunga una buona qualità dell'iter diagnostico ed il giudizio valutativo del processo assistenziale nel suo complesso. La cartella digitale, infatti è utile per: i) guidare il medico e l'infermiere nella compilazione corretta della documentazione, ricorrendo anche a campi obbligati o a check list); ii) consentire una migliore interazione e comunicazione tra il personale sanitario all'interno dell'ospedale o

Tabella 2. Il metodo 'CLAP' per la valutazione della qualità della cartella clinica.

\begin{tabular}{ll}
\hline Parametro da considerare & Note \\
\hline Completezza & Registrazione esaustiva dei dati salienti; assenza di omissioni di elementi rilevanti \\
\hline Leggibilità, ordine e correttezza formale & Leggibilità, sequenza logica, sintassi ed ortografia, flusso narrativo \\
\hline Accuratezza & $\begin{array}{l}\text { Precisione e diligenza contestuale ai fatti, registrazione dei dati accurata e tempestiva, coerenza } \\
\text { con standard di riferimento }\end{array}$ \\
\hline Professionalità & $\begin{array}{l}\text { Impiego di strumenti valutativi del paziente validati; comunicazione scritta idonea, evitando } \\
\text { annotazioni non pertinenti, non professionali o offensive }\end{array}$ \\
\hline
\end{tabular}

Tabella 3. Il metodo CR (Chart Review) e CSR (Chart-Stimulated Recall). (Modificata da ${ }^{65}$ ).

\begin{tabular}{|c|c|c|}
\hline $\begin{array}{l}\text { Revisione della documentazione } \\
\text { in cartella clinica(Chart Review) }\end{array}$ & $\begin{array}{l}\text { Discussione del caso con quesiti } \\
\text { specifici (Chart-Stimulated Recall) }\end{array}$ & Feedback \\
\hline $\begin{array}{l}\text { Revisione nella cartella clinica per } \\
\text { estrapolare alcuni dati chiave, in base alla } \\
\text { documentazione esistente, relativamente a: } \\
\text { tracciabilità; } \\
\text { chiarezza; } \\
\text { accuratezza e appropriatezza; } \\
\text { veridicità; } \\
\text { attualità; } \\
\text { pertinenza; } \\
\text { completezza. } \\
\text { Dalla cartella clinica si possono estrapolare } \\
\text { indirettamente il livello delle conoscenze } \\
\text { mediche messe in atto, la qualità della cura } \\
\text { al paziente, le modalità di comunicazione, } \\
\text { la pertinenza delle informazioni riportate }\end{array}$ & $\begin{array}{l}\text { La discussione sul caso è finalizzata a } \\
\text { sondare il pensiero del medico sul } \\
\text { processo di cura del paziente, utilizzando } \\
\text { le domande pertinenti al caso. } \\
\text { In particolare si dovrebbe discutere sui } \\
\text { seguenti argomenti: } \\
\text { i) il processo decisionale diagnostico: } \\
\text { quali caratteristiche della presentazione } \\
\text { del paziente hanno portato al primo } \\
\text { orientamento diagnostico?; } \\
\text { ii) nella formulazione dell'ipotesi } \\
\text { diagnostica iniziale vi erano elementi } \\
\text { di ambiguità o incertezza? Se sì, come } \\
\text { sono state affrontate? Su quali } \\
\text { elementi razionali sono stati richiesti } \\
\text { gli approfondimenti } \\
\text { laboratoristico-strumentali? } \\
\text { Quali altre indagini potevano essere } \\
\text { eseguite e perché non sono state } \\
\text { richieste?; } \\
\text { iii) Sono stati valutati gli aspetti } \\
\text { soggettivi del vissuto di malattia del } \\
\text { paziente, (sentimenti, idee, aspettative) } \\
\text { e come, in base a queste, è stato modificato } \\
\text { il percorso diagnostico-terapeutico } \\
\text { iv) Dovrebbe essere descritto il razionale } \\
\text { delle decisioni assunte relativamente alla } \\
\text { gestione e al trattamento del caso, esplicitar } \\
\text { altresì gli elementi chiave che hanno } \\
\text { influenzato la decisione finale e le modalità } \\
\text { di follow-up e di continuità assistenziale }\end{array}$ & $\begin{array}{l}\text { Dovrebbe essere fornito un feedback verbale } \\
\text { sintetico al medico, finalizzato ad un confronto } \\
\text { fra le prestazioni osservate e lo standard di } \\
\text { riferimento, evidenziano almeno } 3 \text { punti di } \\
\text { forza e } 3 \text { aree di debolezza, su cui } \\
\text { implementare azioni di miglioramento. Queste } \\
\text { possono includere commenti su: } \\
\text { i) presentazione del caso; ii) utilizzo delle } \\
\text { informazioni e capacità di ragionamento } \\
\text { clinico e capacità riflessiva epicritica; iii) uso } \\
\text { della medicina basata sull'evidenza; iv) capacità } \\
\text { di comunicazione con il paziente e la famiglia; } \\
\text { v) la capacità d'interazione del medico con il } \\
\text { sistema di assistenza territoriale. } \\
\text { In sostanza il feeb back dovrebbe consentire } \\
\text { di rispondere a tre semplici domande: } \\
\text { i) dove sto andando (quali sono gli obiettivi)? } \\
\text { ii) come sto andando (quali progressi si } \\
\text { stanno compiendo verso l'obiettivo)? } \\
\text { iii) dove devo andare? (quali attività devono } \\
\text { essere intraprese per fare progressi e } \\
\text { migliorare?) }\end{array}$ \\
\hline
\end{tabular}


dell'intera USL; iii) Implementare il data-base aziendale di informazioni sanitarie sui pazienti; iv) mettere a disposizione dei clinici strumenti automatici di calcolo di indici di severità e score per le diverse patologie, dai parametri rilevati già di routine all'ingresso del paziente; v) costruire lettere di dimissione personalizzate per singolo paziente e per singola patologia indice e/o prevalente.$^{66-68}$ Le cartelle cliniche elettroniche, sia nelle componenti strutturate, ma ancora di più in quelle non strutturate (con note, consulenze ed interazioni tra professionisti, referti specialistici e dati laboratoristici) offrono una quantità di dati rilevante, da poter essere utilizzate nella valutazione del ragionamento diagnostico.

\section{Conclusioni}

Qualsiasi metodo di valutazione del ragionamento clinico è contrassegnato da vantaggi e limiti. La Tabella 4 evidenzia i pro e i contro di alcuni dei metodi di valutazione presentati.

Qualcuno sostiene che il ragionamento clinico è una 'scatola nera' difficile da aprire. ${ }^{10}$ Il ragionamento diagnostico è un processo complesso, non direttamente misurabile, poiché riferito alla sfera soggettiva del pensiero multi-logico di ogni professionista. Esistono numerosi metodi di valutazione del ragionamento clinico, ma garantire e certificare la competenza richiede lo sviluppo di programmi di valutazione che ne affrontino tutte le componenti, idealmente costruite in maniera complementare, proprio per tenere conto della validità, fattibilità, vantaggi/svantaggi di ciascun metodo. Nessuna singola strategia può essere utilizzata per valutare l'accuratezza delle decisioni diagnostiche dell'internista. Se si vogliono ottenere valutazioni utili ed accurate dovrebbero essere utilizzate strategie multiple. La valutazione della capacità di ragionamento clinico deve essere desunta indirettamente, sulla base della documentazione disponibile, dei comportamenti applicati ai singoli casi e da una diretta interazione con il singolo medico. Un programma improntato ad impiegare più strategie integrate potrà fornire risultati migliori per determinare la competenza del medico. Senza dimenticare i limiti posti dallo stesso processo di valutazione eterodiretto. I valutatori 'esperti' possono essere influenzati dal loro quadro di riferimento di expertise professionale, in cui le conoscenze personali, le proprie abilità e la specializzazione praticata, oltre che eventuali pregiudizi e/o conflitti personali e/o di scuola, possono condizionare il risultato finale della valutazione della performance di un medico che si sottopone a tale giudizio. In sostanza, restano insolute alcune domande. Sono pertanto necessari programmi di approfondimento e di ricerca su questa problematica, ${ }^{69}$ considerandone i benefici in confronto ai costi. Su queste tematiche le Società Scientifiche di Medicina Interna potranno impegnarsi insieme nella ricerca di strumenti validati per la valutazione complessiva delle modalità di ragionamento clinico nei nostri reparti.

\section{Post scriptum}

Nel manoscritto abbiamo volute mantenere diversi termini inglesi originali 'virgolettati' o in corsivo non per una auspicata anglofilia o ripudio della lingua italiana. La lingua inglese domina la letteratura scientifica medica di qualità. Abbiamo voluto facilitare la lettura delle principali parole chiave sul tema e dei molti articoli di riferimento, con un glossario pertinente all'argomento affrontato.

Tabella 4. Pro e contro di alcuni dei metodi di valutazione proposti.

\begin{tabular}{|c|c|c|}
\hline Metodo & Pro & Contro \\
\hline MCQs & $\begin{array}{l}\text { - Elevata validità, affidabilità e fattibilità } \\
\text { - Le modalità dei test sono familiare sia agli } \\
\text { esaminandi che agli esaminatori }\end{array}$ & $\begin{array}{l}\text { - Devono essere rispettate alcune regole fondamentali }{ }^{13} \\
\text { - Si basano quasi sempre su un modello lieare di ragonamento } \\
\text { clinicità } \\
\text { - La specificità può essere bassa }\end{array}$ \\
\hline OCSE & $\begin{array}{l}\text { - L'uso di casi 'reali' consente di ottenere un } \\
\text { elevato del test } \\
\text { - Permette l'esame dell'intero caso }\end{array}$ & $\begin{array}{l}\text { - Richiede molte risorse } \\
\text { - Può essere di bassa affidabilità e troppo soggettiva } \\
\text { - La competenza verificata in un contesto non è predittivo della } \\
\text { competenza generale }\end{array}$ \\
\hline $\begin{array}{l}\text { SCT - Script concordance } \\
\text { test }\end{array}$ & $\begin{array}{l}\text { - Buona specificità } \\
\text { - Consente ampi margini di ragionameno } \\
\text { clinico }\end{array}$ & $\begin{array}{l}\text { - Difficile stabilirne l'affidabilità e validità } \\
\text { - Difficile definire esperti in questo ambito }\end{array}$ \\
\hline Key features test & $\begin{array}{l}\text { - Buona specificità } \\
\text { - Permette di considerare casi non lineari } \\
\text { - Si concentra sugli aspetti difficili del } \\
\text { ragionamento clinico } \\
\text { - Utile per scegliere le indagini più } \\
\text { appropriate, essenziali ed economicamente } \\
\text { più vantaggiose per un'adeguata gestione } \\
\text { dei problemi del paziente }\end{array}$ & $\begin{array}{l}\text { - Validità e affidabilità sono dipendentisul corretto progetto } \\
\text { - dell'esamee numero di caso di grandi dimensioni }(>40)\end{array}$ \\
\hline
\end{tabular}


Appendice 1. Esempio di KFP - key feature problems.

\section{ESEMPIO DI KFP}

KFP 1

Paziente di 56 anni, iperteso con scarsa compliance terapeutica, obeso, forte fumatore con consumo giornaliero di $\mathbf{4 0}$ sigarette/die da $\mathbf{4 0}$ anni; recentemente ha presentato un quadro di bronchite acuta con febbre e dispnea che lo hanno costretto a letto per circa una settimana. Dopo circa una settimana dalla guarigione il paziente presenta un episodio di dolore toracico associato a dispnea. All'arrivo del $118 \mathrm{i}$ parametri sono: PA $95 / 60 \mathrm{mmHg}$, SO2 92\%AA, FC 110 bpm.

Domanda 1:

All'arrivo in pronto soccorso quali esami richiedereste urgentemente per orientarvi su una diagnosi? Potete chiedere massimo 6 esami.
A. Emocromo (1)
B. PCR (1)
C. Creatinina (2)
D. Profilo coagulativo (1)
E. Troponina (2)
F. D-Dimero (1)
G. BNP (2)
H. Emogasanalisi arteriosa (2)
I. ECG (2)
J. Rx torace (1)
K. TC torace senza mdc (1)
L. TC cranio senza mdc (0)
M. Ecocardiografia (2)
N. Ecocolordoppler arti inferiori (1)

Domanda 2 - Gli esami richiesti evidenziano i segenti reperti:

ECG: FC 110 bpm, BBD, aletrazioni secondarie della ripolarizzazione

A. Emocromo: $13000 \mathrm{~GB}, 17 \mathrm{Hb}, 400000 \mathrm{PLT}$

Troponina hs: 100 (range <15)

Creatinina: $4 \mathrm{mg} / \mathrm{dl}$

EGA: pO2 $50 \mathrm{mmHg}$, pCO2 $35 \mathrm{mmHg}, \mathrm{HCO} 3-18, \mathrm{pH} 7,40$

PCR: 60 (range 0-6)

DDimero: 1000

BNP: 490 (range <500)

Rx torace: congestione ilare con addensamento in sede basale destra

In base a tali esami quale tra queste proponete come vostra ipotesi diagnostica?

(Possibili 2 opzioni)
A. Dissezione aortica (1)
B. Trombosi venosa profonda arti inferiori (1)
C. Embolia polmonare (2)
D. Ipertensione polomonare severa (1)
E. IMA destro (2)
F. Polmonite basale destra (1)
G. Edema polmonare acuto (1) 
In considerazione degli esami eseguiti e dei risultati evidenziati, nel caso di diagnosi di embolia polmonare, quale sarebbe la terapia di scelta? Possibile 1 opzione
A. Fondaparinux (0)
B. Enoxaparina (1)
C. Eparina sodica (2)
D. DOAc (0)
E. Warfarin (1)
F. Trombectomia meccanica (0)
G. Trombolisi (1)

\section{COMMENTO ALLA TIPOLOGIA DI TEST}

Quali sono le "caratteristiche chiave" nel processo decisionale in questo caso?

Partendo da un caso clinico iniziale, una KFP question si può estrinsecare in due o più snodi che vanno ad individuare l'appropriatezza del medico nelle scelte diagnostiche e terapeutiche.

Ad ogni possibilità corrisponde un valore che indica quanto quella risposta sia appropriata nel contesto di un adeguata scelta clinica.

Avendo più risposte possibili, è necessario un ragionamento fine che si basi sia sulle conoscenze, sia sul metodo di ragionamento, nonchè sulle nozioni di "clinical governance" nel risolvere problemi spesso molto complessi, considerando anche il rapporto costo/beneficio.

Appendice 2. Esempio di SCT - Script concordance test.

\section{Esempio SCT}

Relazioni tra i passaggi chiave di ragionamento clinico e lo schema SCT

$$
\begin{gathered}
\text { Ragionamento } \\
\text { clinico, passaggi chiave }
\end{gathered}
$$

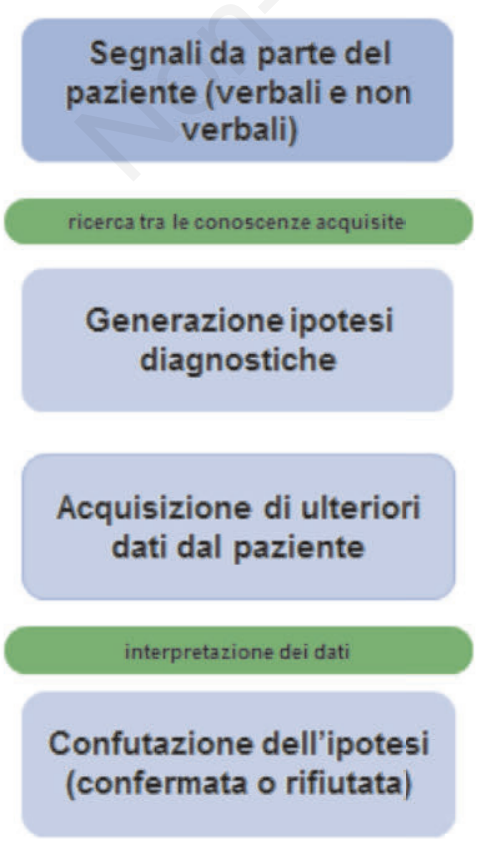

\section{Script Concordance Test}

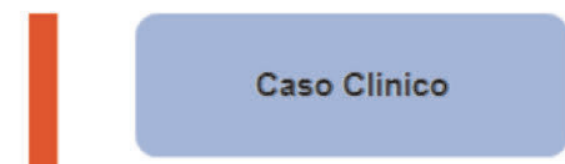

ricerca tra le conoscenze acquisite

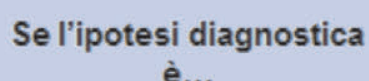

è...

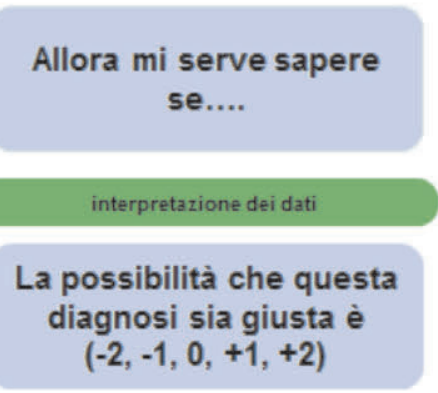


Una paziente entra in ambulatorio. E' una giovane donna che appare abbastanza sofferente e a disagio. Riferisce da tre giorni comparsa di cefalea che interessa l'emilato sinistro del capo, descrivendo il dolore come severo, quasi invalidante.

IPOTESI DIAGNOSTICHE: EMICRANIA, CEFALEA MUSCOLO TENSIVA, NEVRALGIA DEL TRIGEMINO

Segni verbali (cefalea emilato sinistro, dolore di intensità severa) e non verbali (giovane donna, sofferente)

Successivamente essendoci orientati verso un quadro di emicrania chiediamo alla paziente se presenta inoltre sintomi quali nausea e fotofobia, e se questa si presenta omolateralmente alla regione dolente.

Considerato il nostro orientamento verso la diagnosi di emicrania tendiamo a fare domande che confutino la nostra tesi iniziale, domande che fanno parte del copione che è presente nel nostro bagaglio mnemonico su quella specifica patologia.

In un secondo momento mentre ci accingiamo a visitare la paziente notiamo che questa è febbrile $\left(T A>39^{\circ} \mathrm{C}\right)$ e che presenta rigidità nucale.

\section{IPOTESI DIAGNOSTICHE:MENINGITE INFETTIVA, ALTRO STATO INFETTIVO}

Nella nostra mente l'ipotesi diagnostica cambia repentinamente con l'inserimento dei due nuovi dati (febbre e rigidità nucale) e ci porta verso un altro copione, quello della meningite. A questo punto le successive domande saranno stavolta orientate a confutare questa nuova ipotesi diagnostica.

Chiediamo quindi alla paziente se per caso abbia avuto problematiche infettive a carico delle alte vie respiratorie $o$ altro tipo di infezioni nei giorni precedenti.

Lei ci riferisce ora di aver avuto un dolore a carico dell'orecchio sinistro con riduzione dell'udito per qualche giorno e febbricola serotina, ma che il tutto si sarebbe poi risolto spontaneamente.

A questo punto la scelta si fonda sugli esami che possono confutare o meno la nostra ipotesi diagnostica: in prima istanza uno screening ematochimico e successivamente una TC cranio seguita eventualmente da una rachicentesi.

\section{CONFUTAZIONE DELL'IPOTESI DIAGNOSTICA: ACCETTATA O RIFIUTATA}

\section{Commento}

L'esempio sopra riportato rende conto di un modello di ragionamento dove con il modello di condivisione tra conoscenza ed esperienza vengono ad aprirsi diversi cassetti nella nostra mente che man mano si chiudono o si riaprono nel momento in cui noi acquisiamo nuovi dati o confutiamo con gli strumenti a disposizione le nostre ipotesi. 


\section{ESEMPIO SJT}

\section{ESEMPIO DISJT}

Sei un medico internista che viene chiamato in consulenza in ostetricia e ginecologia. Una paziente nel reparto ha una copiosa emorragia postpartum. Negli ultimi minuti è peggiorata clinicamente, divenendo pallida, non responsiva, continuando a sanguinare. Leggendo le sue note, scopri che è una Testimone di Geova e ha firmato un modulo dove si afferma che la donna rifiuta tutti i prodotti del sangue, anche se la sua vita è minacciata. L'ostetrica che si prende cura di lei ha chiesto alla paziente, qualche minuto prima, se avrebbe accettato prodotti sanguigni in caso di minaccia alla sua vita, ma ella ha confermato il suo diniego. Suo marito chiede di fare tutto il possibile per salvare la moglie, anche se questo significa darle il sangue.

\section{Scegli le TRE azioni più appropriate da adottare in questa situazione.}

\section{A. Somministrare emoderivati in quanto si tratta di un'emergenza e potrebbero essere salvavita B. Non dare emoderivati anche se ciò significa che in questo modo la prognosi sarà inevitabilmente infausta \\ C. Metti da parte i desideri del paziente poiché la sua decisione suggerisce una mancanza di capacità decisionale \\ D. Spiega al marito che devi rispettare la decisione del paziente \\ E. Chiedere al marito di dare un consenso formale a nome di sua moglie per fornire emoderivati \\ F. Fai tutto il possibile per salvare la vita della paziente \\ G. Non fare nient'altro dato che morirà inevitabilmente senza la somministrazione di emoderivati \\ H. Contatti il tuo primario e gli chiedi il permesso di somministrare emoderivati nel miglior interesse del paziente}

\section{COMMENTO ALLE POSSIBILI RISPOSTE}

Questa domanda valuta la capacità di far fronte alla pressione, concentrarsi sul paziente e comunicare con parenti e colleghi.

Spiegare al marito che devi rispettare la decisione del paziente assicura che sia informato della tua decisione e che capisca la il fondamento delle tue azioni (D). Avete sia una conferma scritta della direttiva avanzata del paziente che non vuole che le siano somministrati derivati del sangue esattamente in questo scenario, sia una conferma da parte di un collega coinvolto nella cura del paziente che non aveva cambiato idea nonostante il sopraggiungere dell'emergenza. In questa situazione, dovresti rispettare i desideri del paziente e non dare derivati del sangue anche se questo la espone a pericolo di vita (B). Ciò si basa sulla guida GMC che recita 'Se un paziente manca di capacità e le informazioni su un rifiuto avanzato scritto o verbale del trattamento sono registrate nelle loro volontà o sono altrimenti portate alla vostra attenzione, dovete tenere presente che i rifiuti avanzati validi e applicabili devono essere rispettati. ' (Guida GMC del fine vita). Sebbene questo paziente abbia rifiutato i prodotti ematici, dovresti comunque fare tutto il possibile per salvarti la vita (F) come parte dei doveri fondamentali di un medico. 'Devi fare della cura del tuo paziente la tua prima preoccupazione' (GMC Good Medical Pratice).

Anche se dare i derivati del sangue come salvavita può sembrare un'opzione ragionevole (A), in questo scenario hai un rifiuto anticipato scritto e una conferma verbale da parte di un collega che il paziente non aveva cambiato idea, si andrebbe contro le volontà scritte della paziente, che vanno comunque rispettate. È importante che i medici rispettino le convinzioni e le decisioni dei pazienti anche se possono non sembrare logiche per noi sarebbe sbagliato attribuire una mancanza di capacità semplicemente perché la scelta del paziente è diversa dalle convinzioni di un medico (siano esse di tipo religioso o altro) (C). Un marito non può dare il consenso a nome di sua moglie (E) laddove una paziente non è in grado di prendere una decisione, ne i medici ne tanto meno $\mathrm{i}$ membri anziani dovrebbero prendere in considerazione di andare contro il volere del paziente (ad esempio il rifiuto anticipato scritto) seppur nei migliori interessi della stessa. In questo caso, poiché vi è una chiara evidenza dei desideri del paziente, chiedere il permesso al proprio primario $(\mathrm{H})$ non sarebbe utile in quanto esso dovrebbe comunque attenersi alle volontà della paziente. Questa paziente non ha rifiutato la rianimazione o qualsiasi altra forma di aiuto ma solo gli emoderivati, quindi sarebbe negligente non fare nient'altro $(\mathrm{G})$. 
Appendice 4. Esempio di un caso KFQs - Key-feature questions. ${ }^{70}$

\section{ESEMPIO DI KFQS}

Paziente di $\mathbf{7 6}$ anni, arriva in pronto soccorso con dolore a carico della gamba destra che appare tumefatta e dolente, mentre la sinistra non lamenta alcun problema. La sintomatologia dura da almeno due giorni e progressivamente sta peggiorando.

\section{Domanda 1:}

Quali diagnosi prendi in considerazione? Puoi elencarne due.

A.

Key feature 1: il candidato deve inserire tra le due diagnosi TROMBOSI VENOSA PROFONDA

Scoring Key:

SCORE

1

0

\section{CRITERI}

trombosi venosa profonda inserire più di due risposte

\section{SINONIMI}

DVT

KFQs 2

A. Assunzione di alcolici

B. Allergie

C. Storia di angina pectoris

D. Assunzione di antinfiammatori

E. Fumo di sigaretta

F. Colore delle feci

G. Tosse

H. Cefalea

I. Ematemesi

J. Impotenza

K. Claudicatio intermittens

L. Dolore lombare

M. Nicturia

N. Palpitazioni

O. Parestesie

P. Dispnea parossistica notturna

Q. Polidipsia

R. Pregressi problemi al rachide

S. Pregressi problemi alle ginocchia

T. Pregressa neoplasia

U. Pregressa infezione del tratto urinario

V. Recenti procedure odontoiatriche

W. Recenti voli aerei intercontinentali

$X$. Recente faringodinia

Y. Recente chirurgia

Z. Recenti condizioni di lavoro

AA. Non sono necessarie altre informazioni 
Key feature 2: il candidato deve elencare i fattori di rischio per TROMBOSI VENOSA PROFONDA

Scoring Key:

SCORE

CRITERI

1. Devi elencare almeno cinque dei seguenti sei elementi:

E. Fumo di sigaretta

S. Pregressi problemi alle ginocchia

T. Pregressa neoplasia

W. Recenti voli aerei intercontinentali

Y. Recente chirurgia

Z. Recenti condizioni di lavoro

$0 \quad$ Non selezionare almeno cinque dei precedenti sei elementi oppure

selezionare la risposta AA "non sono necessarie ulteriori informazioni"

oppure

selezionare più di sette elementi

Appendice 5. Esempio di un caso IDEA - Interpretive summary, Differential diagnosis, Explanation of reasoning, and Alternatives. ${ }^{71,72}$

I.D.E.A.

Interpretive summary, Differential diagnosis, Explanation of reasoning, and Alternatives

In quale di questi scenari è più appropriata la gestione del paziente al domicilio?
A. Uomo di 80 anni con CAP e confusione mentale
B. Uomo di 85 anni con CAP e frequenza respiratoria di 22 atti/min
C. Donna di 70 anni con CAP e urea di $50 \mathrm{mg} / \mathrm{L}$ ( $18 \mathrm{mmol} / \mathrm{L}$ )
D. Donna di 65 anni con CAP, PAS $80 \mathrm{mmHg}$ e disorientata

\section{SPIEGAZIONE DELLE RISPOSTE APPROPRIATE E NON APPROPRIATE} (SECONDO EVIDENZE)
A. 2 punti (medical ward-reparti di Medicina Interna)
B. 1 punto: outpatient (terapia al domicilio)
C. 2 punti (medical ward- reparti di Medicina Interna)
D. 3 punti: ICU (reparti Intensivi/Semintensivi) 


\section{Mini-Oinical Evaluation Exercis (CEX) - Versione F1}

Barrare con X
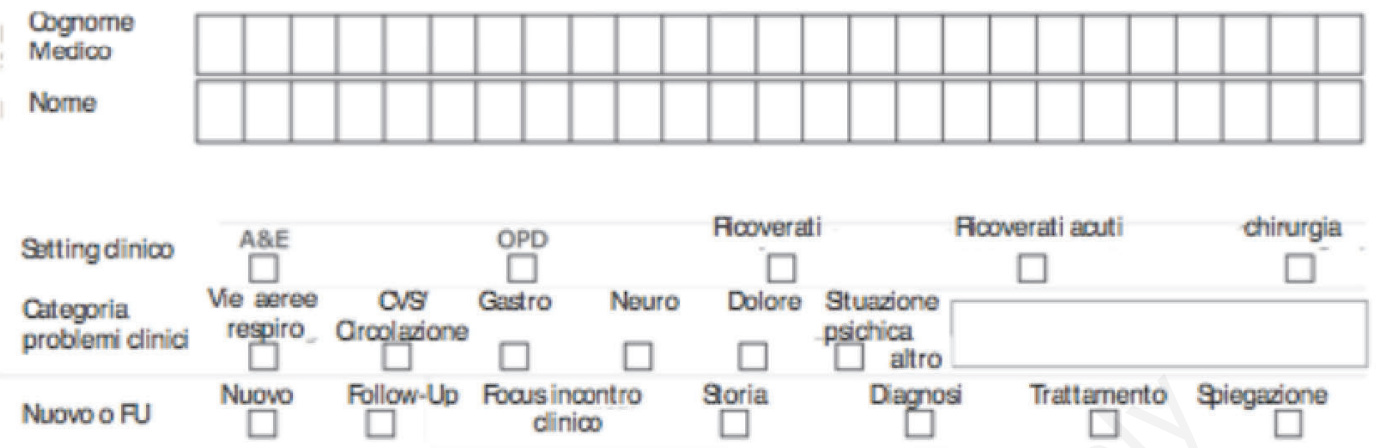

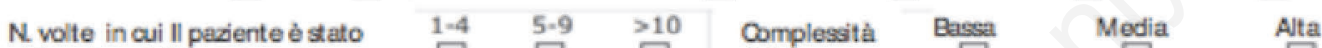
visto nrima dall'peminando $\quad \square \quad \square \quad \square \quad$ del caso

Posizione Consulente

del valutatore $\quad \square \quad$ Altro

Numero di mini-CXXprecedenti osservati dal valutatore con l'esaminando

Valutare le seguenti aree utilizando la scala seguente

1. Racoolta della storia

\section{Abilità nell'esame fisico}

3. Capacità comunicative

\section{Gudizio dinico}

\section{Professionalità}

6. Organizzazione/efficienza

\section{Assistenza clinica generale}

* Non è possibile commentare

\section{Segnalazioni particolarmente significative}

\section{Suggerimenti}

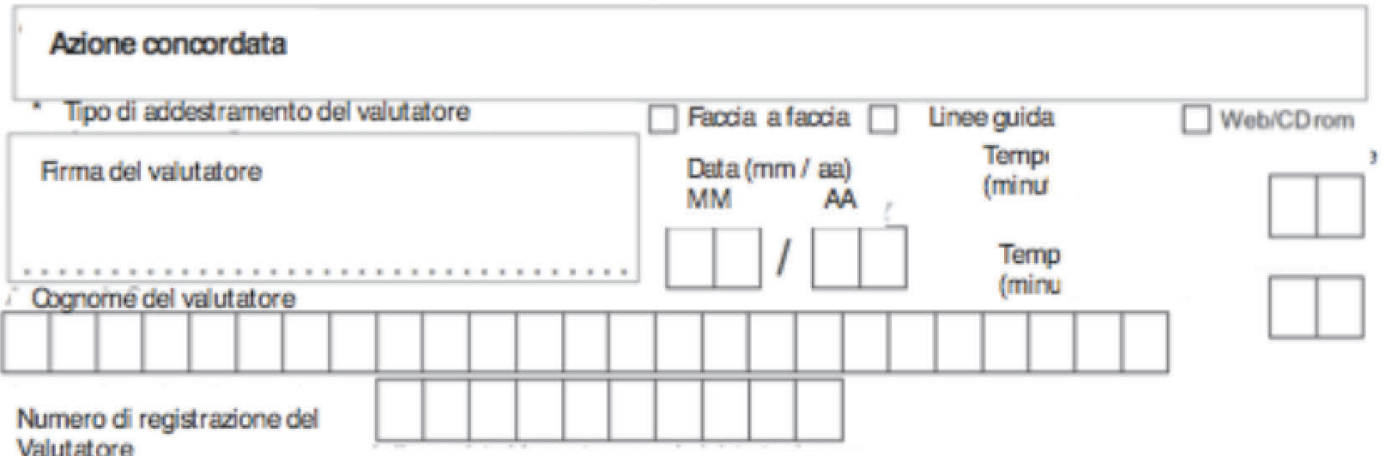

OPD, Outpatient department 
Osservazione diretta delle abilità procedurali (DOPS) - Versione F1.

Oognome

Medico

Nome ,
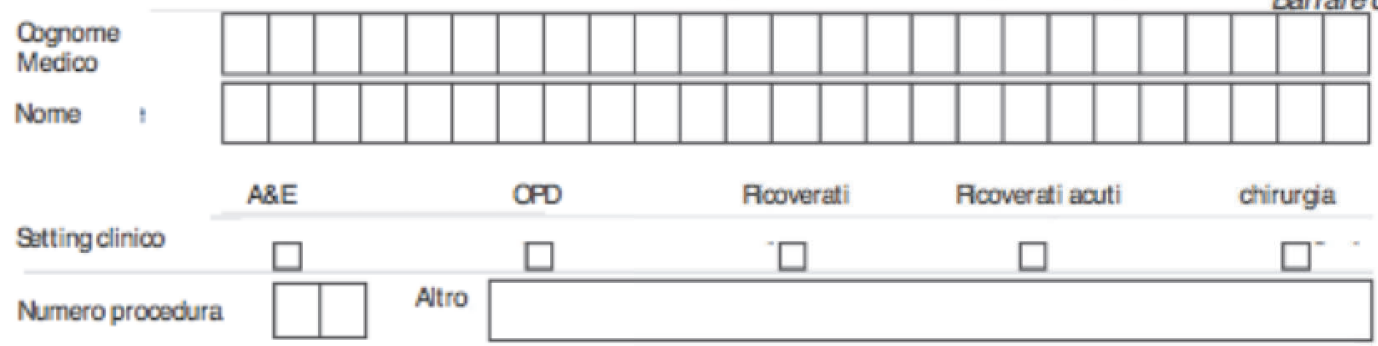

\section{Posizione \\ del valutatore \\ $\square$ Consulente \\ $\square$ Altro (specificare)}

\section{Numero di precadenti DOPSosservati dal} valutatore con ocni esaminando

Numero di procedure

$\begin{array}{llll}0 & 1-4 & \square & \square \\ \square & \square & \square\end{array}$

effettuate dal'esaminando $\square$

Valutare le seguenti aree

usando la scala

anatomia pertinente, tecrica di procedura

2 Ottiene il consenso informato

3 Dimostra appropriata preparazione

pre-procedura

4. Analgesia appropriata o sedazione sicura

5. Abilità tecrica

6. Tecrica asepsi

7. Cerca aiuto se è il caso

8. Gestione postprocedurale

9. Capacità comunicative

10. Considerazione del paz' professionalità

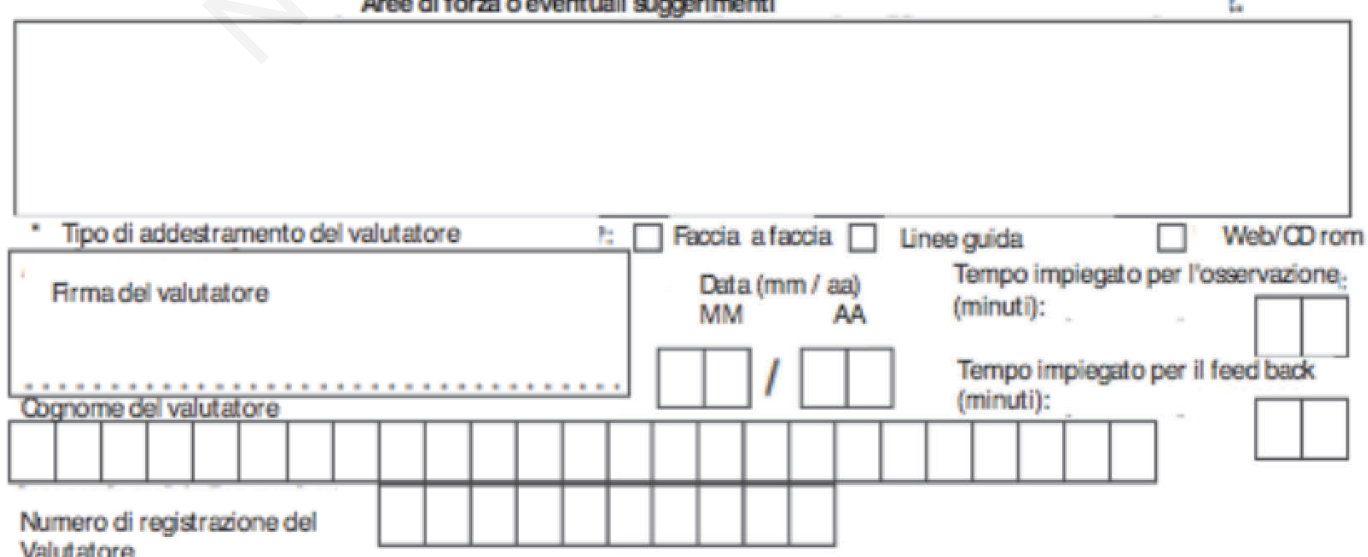




\section{Bibliografia}

1. Norcini J, Burch V. Workplace-based assessment as an educational tool, AMEE GUIDE Assessment 31, Association for Medical Education in Europe (AMEE), Dundee, UK, Scottish Charity no SC 031618, C AMEE 2007.

2. Epstein RM, Assessment in Medical Education, N Engl J Med 2007;356:387-936.

3. Holmboe ES, Sherbino J, Long DM, Swing SR, Frank JR. The role of assessment in competency-based medical education, Med Teach 2010; 32/8: 676-682.

4. Consorti F. Valutazione e autovalutazione: due facce della stessa medaglia, Società Italiana di Pedagogia Medica, SIPeM, Ferrara 25 settembre 2018.

5. Mazzone A, Nozzoli C, Berti F, Colombo F, Filannino C, Greco A, Mathieu G, Mattarei M, Nardi R, Stornello M. The Clinical competence in Internal Medicine, Italian Journal of Medicine 2013, 5/3, s1, S5-S28. https://doi.org/10.4081/itjm.2011.3.s1

6. Bowen JL. Educational Strategies to Promote Clinical Diagnostic Reasoning, Cox M. Irby DM Editors, N Engl J Med 2006; 355: 2217-2225.

7. Mathieu G, Greco A, Nardi R et al. La clinical competence in Medicina Interna, Italian Journal of Medicine 2011, 5S, S17-S29.

8. Hege I, Kononowicz AA, Kiesewetter J, Foster-Johnson L. Uncovering the relation between clinical reasoning and diagnostic accuracy - An analysis of learner's clinical reasoning processes in virtual patients. PLoS ONE 2018, 13/10: e0204900. https://doi.org/10.1371/ journal.pone. 0204900

9. ABIM-American Board of Internal Medicine, http://www.abim.org/program-directors-administrators /assessment-tools/mini-cex.aspx

10. Ilgen JS, Humbert AJ, Khun G et al. Assessing Diagnostic Reasoning: a consensus statement summarizing theory, practice, and future needs, Academic Emergency Medicine 2012, 19/2: 1454-1461.

11. Bianchi L, Gallagher EJ, Korte R, Ham HP. Interexaminer agreement on the American Board of Emergency Medicine oral certification examination, Ann Emerg Med 2003, 41: 859-864.

12. Maatsch JL. Assessment of clinical competence on the Emergency Medicine Specialty Certification Examination: the validity of examiner ratings of simulated clinical encounters. Ann Emerg Med 1981, 10: 504-507.

13. Tangianu F, Mazzone A, Berti F, Pinna G et al. Are multiple-choice questions a good tool for the assessment of clinical competence in Internal Medicine? Italian Journal of Medicine 2018, 12/2: 88-96.

14. Farmer EA, Page G. A practical guide to assessing clinical decision-making skills using the key features approach, Medical education 2005, 39/12: 1188-1194.

15. Consorti F. Didattica professionalizzante nei corsi di laurea in medicina, Edra, laFeltrinelli 2018.

16. Hrynchak PK, Takahashi SG, Nayer M. Key-feature questions for assessment of clinical reasoning: A literature review, Medical Education 2014, 48/9: 870883

17. Kwa SK, Sheikh Mohd Amin, Ng AC. Avoiding common errors in Key Feature Problems, Malaysian Family Physician. 2007, 2/1: 18-21.

18. McGrath M. https://leanforward.hms.harvard.edu/ 2017/10/24/clinical-reasoning-storing-stories-to-narrow -the-diagnosis/, 24 ottobre 2017

19. Fournier JP, Demeester A, Charlin B. Script Concordance Tests: Guidelines for Construction, BMC Medical Informatics and Decision Making 2008, 8: 18.

20. Boulouffe C, Doucet B,Muschart X, Charlin B, Vanpee $\mathrm{D}$, Assessing clinical reasoning using a script concordance test with electrocardiogram in an emergency medicine clerkship rotation, Emerg Med J 2014, 31/4: 313-316.

21. Lubarsky S1, Dory V, Duggan P, Gagnon R, Charlin B. Script concordance testing: from theory to practice: AMEE guide no. 75. Med Teach. 2013; 35/3: 184-193.

22. Harden RM. What Is an OSCE? Medical Teacher 1988, 10:19-22.

23. Colombo F. Clinical competence: il problema della valutazione, Fondazione FADOI, Dipartimento Formazione e Aggiornamento - Attività 2011-2017, Booklet Formazione FADOI 2017.

24. Varian F, Cartwrighte L. The Situational judgement test at a glance, Wiley-Blackwell 2012.

25. de Leng WE, Stegers-Jager KM, Born MP. Themmen APN. Integrity situational judgement test for medical school selection: judging 'what to do' versus 'what not to do', Med Educ. 2018, 52/4: 427-437.

26. Plourde P, Gfor the development of key feature problems \& test cases, 2012, https://mcc.ca/media/ CDM-Guidelines.pdf

27. Hrynchak P, Takahashi SG, Nayer M. Key-feature questions for assessment of clinical reasoning: a literature review, Med Educ. 2014, 48/9: 870-883.

28. Keefe S, Johnson E, Pung J. Improving clinical reasoning by transferring the control of the path and the branches of the virtual patient to trainees using a nonlinear, non-branched model, AMEE EdMedPublish 2016, https://doi.org/10.15694/mep.2016.000125

29. Pieralli F. Innovazione nel metodo di fare formazione, il paziente virtuale, in: I Convegno Nazionale sulla Formazione in Sanità, Auditorium Ministero della Salute, Roma 26 settembre 2017.

30. Gaba DM. The future vision of simulation in health care, BMJ Quality \& Safety 2004: i2-i10.

31. Bodamer C, Feldman M, Kushinka J, et al. An Internal Medicine Simulated Practical Examination for Assessment of Clinical Competency in Third-Year Medical Students, Simul Healthc 2015; 10/6): 345351 .

32. Kolb D. Experiential learning: experience as the source of learning and development, Englewood Cliffs, N.J: Prentice-Hall; 1984.

33. Pennaforte T, Moussa A, Loye N, Charlin B, Audétat MC. Exploring a New Simulation Approach to Improve Clinical Reasoning Teaching and Assessment: Randomized Trial Protocol, JMIR Res Protoc 2016; 5/1: e26.

34. Hege I, Kononowicz AA, Adler M. A Clinical Reasoning Tool for Virtual Patients: Design-Based Research Study, JMIR Med Educ 2017, 3/2: e21.

35. Ryall T, Judd BK, Gordon CJ. Simulation-based assessments in health professional education: a 
systematic review, Journal of Multidisciplinary Healthcare 2016:9 69-82.

36. Dillon GF, Clauser BE. Computer-delivered patient simulations in the United States Medical Licensing Examination (USMLE), Simul Healthc 2009; 4: 30-34.

37. Huang G, Reynolds R, Candler C. Virtual patient simulation at US and Canadian medical schools, Acad Med 2007; 82:446-451.

38. Harik P, Cuddy MM, O'Donovan S et al. Assessing potentially dangerous medical actions with the computer-based case simulation portion of the USMLE step 3 examination, Acad Med 2009; 84: S79-82.

39. Murphy JG, Cremonini F, Kane GC, Dunn W. Is simulation based medicine training the future of clinical medicine? European Review for Medical and Pharmacological Sciences 2007, 11: 1-8.

40. Baker E, Ledford C, Fogg L et al. The IDEA Assessment Tool: Assessing the Reporting, Diagnostic Reasoning, and Decision-Making Skills Demonstrated in Medical Students' Hospital Admission Notes, Teaching and Learning in Medicine 2015, 27/2: 163-173.

41. Armenia S, Thangamathesvaran L, Caine AD, King N, Kunac A, Merchant AM. The Role of High-Fidelity Team-Based Simulation in Acute Care Settings: A Systematic Review, Surg J (N Y) 2018; 4/3: e136-e151.

42. Liu C. An introduction to workplace-based assessments, Gastroenterol Hepatol Bed Bench. 2012, 5/1: 24.

43. ABIM-American Board of Internal Medicine, Mini CEX, https://www.abim.org/program-directors-administrators/ assessment-tools/mini-cex.aspx

44. Holmboe ES, Huot SJ, Chung J, Norcini JJ, Hawkins RE. Construct validity of the mini-clinical evaluation exercise -mini-CEX. Acad Med 2003, 78: 826-830.

45. Nardi R, Mathieu G, Berti F, Filannino C, Greco A, Nozzoli C, Mazzone A a cura del gruppo di lavoro FADOI-SDA Bocconi Modelli di valutazione della clinical competence del medico specialista internista ospedaliero, Italian Journal of Medicine, 2011, 5S: S33-S45.

46. Mazzone A, Colombo F, Croce D, Nardi R. "Bedside teaching": competenza clinica e formazione sul campo. Torniamo all' antico per essere moderni, Italian Journal of Medicine 2011, 5S, S30-S32.

47. Rider E, Nawotniak R, Smith G. A Practical Guide to Teaching and Assessing the ACGME Core Competencies, 2007, Marblehead (MA), HCPro, Inc.

48. Violato C, Lockyer J, Fidler H. Multisource feedback: a method of assessing surgical practice, BMJ 2003; 326/7388: 546-548.

49. Sidney W. Multisource Feedback Problems and Potential, Academic Medicine 2013, 88/8: 1055.

50. Narayanan A, Farmer EA, Greco MJ. Multisource feedback as part of the Medical Board of Australia's Professional Performance Framework: outcomes from a preliminary study, BMC Med Educ 2018, 18/1: 323-. Published 2018 Dec 29. doi:10.1186/s12909-018-1432-7

51. Clin. Gov. Support Team, A Practical Handbook for Clinical Audit - March 2005.

52. Adamo C, Wienand U. Audit Clinico, 2008, http://www.ospfe.it/il-professionista/progetto-iqip/allegati /audit_clinico_breve_descrizione.pdf

53. Benjamin A. Audit: how to do it in practice BMJ 2008; 336; 1241-1245.

54. NICE, CHI, RCN, University Leicester Principles for best practice in Clinical Audit; Radcliffe Medical Press, 2002.

55. Ministero della Salute, Manuale 'L' Audit Clinico' 2011.

56. Ebook: Le raccomandazioni della Siquas - Vrq sull'Audit Clinico - Che cosa dice la letteratura? 2014.

57. Patil SY, Hashilkar NK, Hungund BR. Blueprinting in assessment: how much is imprinted in our practice? Journal of Educational Research and Medical Teacher 2014, 2/1: 4-6.

58. Adkoli BV, Deepak KK. Blue printing in assessment. In: Singh T, Anshu, editors. Principles of assessment in medical education. New Delhi: Jaypee Publishers; 2012, pp. 205-213.

59. Gujarathi A. Dhakne-Palwe S et al, Preparation of Blue Prints for formative Theory Assessment of Undergraduate Medical Students in Community Medicine. MVP Journal Of Medical Science 2015, 2/2: 100-103.

60. Patil SY, Gosavi M, Bannur HB, Ratnakar A. Blueprinting in assessment: a tool to increase the validity of undergraduate written examinations in pathology, International Journal of Applied and Basic Medical Research 2015, 5/Suppl 1): S76-79.

61. The America Board of Internal Medicine, https://www.abim.org/ /media/ABIM\%20Public/Files/p df/exam-blueprints/certification/internal-medicine.pdf

62. Università degli Studi del Piemonte Orientale, Clinica Medica -Anni 2011-2012, https://www.dir.uniupo.it/ pluginfile.php/199062/mod_resource/content/1/Introduz ione $\% 20 \mathrm{al} \% 20$ corso.pdf)

63. Beck BG. CLAPprinciplesof medical record documentation, CME-Location Seminar, July 1988.

64. Nardi R. Cipolla D'Abbruzzo C, La responsabilità del medico in Medina d'urgenza e Pronto Soccorso, Centro Scientifico Editore, Torino, 1996.

65. Philibert I. Using Chart Review and Chart-Stimulated Recall for Resident Assessment, Journal of Graduate Medical Education, 2018, 10/1: 95-96, DOI: http://dx.doi.org/10.4300/JGME-D-17-01010.1

66. Van der Ridder JM, Stokking KM, McGaghie WC et al. What is feedback in clinical education?, Med Educ 2008, 42: 189-197.

67. Hattie J, Timperley H. The Power of Feedback, . Review of Educational Research. 2007; 77: 81-112.

68. Chesi G, Maccari S, Ligabue R, Messori A, Nicolini F. Cartella clinica informatizzata per un Dipartimento di area internistica organizzato per intensità di cure: strumento per migliorare l'appropriatezza e la qualità delle cure sanitarie, Politiche sanitarie 2012, 13/2: 109124.

69. Warren A. Integrating teaching and assessment of clinical reasoning, https://fvas.unimelb.edu.au/_data/ assets/pdf file/0011/2806508/900-Warren-Integratingteaching-and-assessment-of-clinical-reasoning-FINAL EB-notes.pdf

70. Daniel M, Rencic J, Durning S et al. Clinical Reasoning Assessment Methods, A Scoping Review and Practical Guidance, Academic Medicine 2019, January, Volume Publish Ahead of Print - Issue - p, doi: 10.1097/ACM.0000000000002618

71. Schuwirth LW, Van der Vleuten CP. Programmatic assessment: from assessment of learning to assessment for learning, Medical teacher 2011, 33/6: 478-485. 
72. Lubarsky S, Dory VR, Duggan P, Gagnon R, Charlin B. From theory to practice: AMEE Guide No. 75.

73. GMC-General Medical Council, Treatment and care towards the end of life: good practice in decision making, 2010, ISBN: 978-0-901458.

74. GMC-General Medical Council, Good medical practice, www.gmc-uk.org/guidance, 2013.

75. http://emedica.co.uk/sjtquestions.html

76. Plourde P, Chair, MCC CDM Examination Committee, Guidelines for the development of key feature problems
\& test cases, Medical Council of Canada, 2012, https:// mcc.ca/media/CDM-Guidelines.pdf

77. Holmboe ES, Durning SJ, Hawkins RE. Practical Guide to the Evaluation of Clinical Competence E-Book, Elsevier, $2^{\text {nd }}$ Edition, 2018.

78. Lim WS, van der Eerden MM, Laing $\mathrm{R}$ et al. Community acquired pneumonia severity on presentation to hospital: an international derivation and validation study. Thorax 2003; 58: 377. 


\title{
La semeiotica medica: presente e futuro
}

\author{
Francesco Cipollini \\ Scuola di Ecografia Internistica FADOI, UO Medicina Interna, Clinica Villa Verde, Fermo, Italia
}

\section{Cenni storici}

La semeiotica medica, ovvero il rilievo dei segni che orientano la diagnosi, prima del XVIII secolo si limitava alla ispezione per la ricerca di manifestazioni cutanee, alla valutazione del polso (già praticato dalla medicina cinese), alla osservazione delle urine (già descritta sin dal X secolo dalla Scuola Medica Salernitana). L'inizio della ricerca e dello studio di segni, potenzialmente in grado di valutare gli 'organi interni', che segnano l'inizio della semeiotica medica moderna coincide con l'opera di Leopold Auenbrugger (1722-1808) pubblicata nel 1761 dal titolo 'Unventum Novum Ex Percussione Thoracis Humani ut Signo'. ${ }^{1}$ Partendo dall'osservazione che l'oste con la percussione delle botti valutava il livello del vino, applicò la percussione al torace per valutare la presenza di versamento pleurico. Purtroppo la sua scoperta rimase negletta e fu riscoperta, rivalutata e promossa da Corvisart, medico personale di Napoleone Bonaparte agli inizi dell' 800 .Quasi un secolo dopo R. Laennec (1781-1826) ispirato da due fanciulli che comunicavano utilizzando un pezzo di legno, sviluppò lo stetoscopio e usò questo nuovo strumento, che ha caratterizzato la semeiotica medica degli ultimi due secoli, per investigare i suoni provenienti dal cuore e dai polmoni. Laennec pubblicò queste osservazioni nel 1819 nel testo De l'Auscultation médiate ou traité du diagnostic des maladies des poumons et du cœur. ${ }^{2}$ Questa 'auscultazione mediata' era considerata all'epoca rivoluzionaria perchè invece di poggiare l'orecchio direttamente sul torace per ascoltare il cuore ed i

Corrispondente: Francesco Cipollini, Scuola di Ecografia Internistica FADOI, UO Medicina Interna, Clinica Villa Verde, Fermo, Italia.

E-mail: francescocipollini@gmail.com

Articolo pubblicato secondo la Creative Commons Attribution NonCommercial 4.0 License (CC BY-NC 4.0).

${ }^{\circ}$ Copyright: the Author(s), 2020

Licensee PAGEPress, Italy

QUADERNI - Italian Journal of Medicine 2020; 8(1):125-135 polmoni (pratica che era già in uso sin dalla medicina ippocratica), l'ascoltazione era mediata e amplificata dallo stetoscopio. Questo mezzo diagnostico, che subirà evoluzioni tecnologiche negli anni futuri, è stato lo strumento universalmente utilizzato dai medici nei due secoli successivi (lo scorso anno si è celebrato il bicentenario della scoperta dello stetoscopio) e rappresenta l'icona della professione medica. Oltre alla ispezione e alla palpazione, la percussione e l'auscultazione (con lo stetoscopio prima ed il fonendoscopio negli anni successivi), hanno rappresentato e rappresentano i fondamentali della semeiotica fisica.

La semiotica medica a partire dagli inizi dell' 800 si è arricchita anche di strumenti che hanno permesso di rilevare e studiare segni clinici importanti per la definizione diagnostica al letto del malato. Karl Wunderlich (1815-1877) pubblica nel $1868^{3}$ 'On the temperature in diseases: a manual of medical thermometry'. Dalle rilevazioni della temperatura corporea rilevata su 25.000 casi definì i limiti della temperatura corporea e le variazioni indicative di specifiche malattie (es. febbre tifoide). Hermann von Helmhlotz (1821-1894) nel 1851 ideò e presentò ${ }^{4}$ l'oftalmoscopio per la valutazione del fundus oculi. Scipione Riva Rocci (1863-1937) nel 1896 introdusse lo sfigmomanometro a bracciale per la valutazione delle pressione arteriosa. ${ }^{5}$

Un altra pietra miliare, che a distanza di due secoli dalla scoperta dello stetoscopio, ha rivoluzionato la semeiotica fisica e strumentale a letto del malato è rappresentato dall'impiego degli ultrasuoni. Il primo impiego sperimentale dell'ultrasonografia in medicina risale al 1932 quando Dussik, uno dei pionieri dell'ecografia, basandosi sul ritardo dell'eco di ritorno visualizzato in A mode pose il sospetto (successivamente confermato) di una neoplasia cerebrale. ${ }^{6} \mathrm{Nel} 1953 \mathrm{~J}$. Wild $^{7}$ per primo diagnosticò in ecografia $\mathrm{B}$ mode una neoplasia mammaria.

\section{Key point}

Dopo la scoperta dello stetoscopio, l'ecografo rappresenta sicuramente l'innovazione più importante per l'approccio diagnostico al letto del malato. 


\section{La semeiotica del XXI secolo: l'esame clinico-ecografico integrato}

Agli albori dell'ecografia le apparecchiature erano ingombranti, con riproduzione di immagini estremamente scadenti. Lo sviluppo tecnologico che c'è stato nella seconda metà del secolo scorso, ha consentito il salto di qualità dell'ecografia in quanto ha permesso di avere a disposizione apparecchiature estremamente contenute di dimensioni ma di ottimo livello tecnologico. Nelle ultime due decadi le aziende hanno prodotto apparecchiature ecografiche sempre più compatte ma di alta qualità e di costo contenuto Questo sviluppo ha permesso lo sviluppo della 'bedside songraphy' ovvero l'ecografia eseguita ed interpretata dal clinico al letto del malato. L'impiego dell'ecografo al letto del malato è già praticato in diverse realtà sia ospedaliere che ambulatoriali soprattutto in quest'ultimo decennio grazie anche alla recente introduzione di apparecchiature miniaturizzate che ha permesso addirittura di disporre non solo di strumenti carrellabili o portatili ( delle dimensioni di un tablet ) ma anche ecografi tascabili da portare come per lo stetoscopio - nella tasca del camice.

Gli AA anglo-sassoni hanno definita questa indagine ecografica al letto del malato Point of care Ultrasound (POCUS). Questa definizione ben sin adatta in ambienti - come i reparti di medicina interna - dove l'indagine ultrasonografica segue ad integrazione e compendio dell'esame clinico. Ma ancor più appropriato appare il termine di Focused Bedside Sonography o ancor meglio Goal-directed proprio a significare la peculiarità dell'indagine eseguita dal clinico rispetto a quella eseguita dagli specialisti dell'imaging. Mentre il medico dell'imaging esegue una ecografia d'organo, il clinico - e l'internista in particolare - esegue una indagine orientata al sintomo. Da alcuni anni in alcuni Corsi di Laurea $^{8}$ si sono implementati,anche per l'esame fisico al letto del malato, modelli di insegnamento orientati al sintomo in luogo di quello tradizionale sistematico. Questo modello definito come l'esame obiettivo guidato all'ipotesi, tende a superare l'esame fisico tradizionale inteso come una lista (peraltro riportata nel format delle nostre cartelle cliniche) di caratteristiche che devono essere descritte in ogni paziente per ogni organo e/o apparato. L'esame obiettivo guidato dall'ipotesi, invece, insegna a sviluppare le abilità nel ragionamento diagnostico. A differenza del tradizionale esame, effettuato in senso cranio-caudale (con il classico metodo: ispezione, palpazione, percussione, auscultazione e manovre particolari aggiuntive della semeiotica tradizionale) l'esame fisico guidato dall'ipotesi, in base ai problemi attivi, utilizza il sintomo specifico del paziente per orientare l'iniziale ipotesi diagnostico-differenziale e procedere alla ricerca di specifici segni compatibili con tale sospetto.

Ormai non vi è settore della medicina ${ }^{9}$ che non utilizzi l'ecografo come mezzo di supporto sia a completamento dell'esame clinico sia come supporto di indagini strumentali c.d. eco-guidate/eco-assistite.

In Medicina Interna la bedside sonography ha profondamente cambiato l'approccio semeiologico. L'indagine eseguita al letto del malato (Tabella 1) permette di dare rapidamente e in modo appropriato risposte ai quesiti clinici del caso in esame. ${ }^{10} \mathrm{Nel}$ modello tradizionale il clinico, dopo aver raccolto la storia ed effettuato l'esame obiettivo, decide di richiedere un'ecografia per confermare/escludere un sospetto clinico. Il paziente viene quindi trasportato in una unità operativa diversa (di solito Dipartimento Radiologia) dove l'esame viene eseguito da un altro medico (es. radiologo), che esegue l'indagine e rinvia con il relativo report (referto) il paziente al medico responsabile del paziente. Una simile procedura comporta inevitabilmente un ritardo nella decisione clinica perché l'ecografia richiesta durante una prima visita, ha bisogno di tempo per essere eseguita, il report deve essere scritto e - dopo un lasso di tempo variabile ma comunque non trascurabile - il paziente ritorna al medico curante che, in base al risultato dell'indagine, con una seconda visita, imposta il successivo l'iter diagnostico e terapeutico. Invece nel caso dell'esame ecografico eseguito al letto del malato ad integrazione dell'esame obiettivo, il clinico esegue direttamente l'esame e sulla base dei reperti sonografici può indirizzare l'iter diagnostico e terapeutico senza perdite di tempo.

L'integrazione ecografica ha sicuramente rivoluzionato la semeiotica fisico-strumentale in campo cardiologico. La semeiotica tradizionale utilizzava la percussione per definire l'aia cardiaca per avere un rilievo generico di aumento delle dimensioni cardiache. Con l'indagine ecografica, è possibile definire non solo se è presente l'aumento volumetrico in toto del cuore ma definire quale/i camere cardiache sono dilatate così come è possibile rilevare se vi sono aumenti di spessore (ipertrofia) e quali segmenti cardiaci (setto, pareti, globale) interessano. Non solo. L'indagine permette anche una visualizzazione diretta della cinetica cardiaca e permette inoltre di valutare mediante software dedicati, ormai disponibili su tutte le apparecchiature anche quelle di piccole dimensioni, la frazione di eiezione del ventricolo sinistro. Con queste semplici valutazioni è possibile differenziare rapidamente e correttamente una cardiopatia ipertrofica da una ipocinetico-dilatativa ed indirizzare in tempi brevi il trattamento più appropriato per il caso in esame. La semeiotica tradizionale mediante l'ascoltazione permetteva la valutazione di soffi le cui caratteristiche (sede, timbro, intensità ed irradiazione) 
orientavano,qualora presenti, sia la sede di provenienza del reperto (valvola mitrale, tricuspide, aortica, polmonare) sia il tipo di alterazione (stenosi, insufficienza, steno-insufficienza) della valvola medesima. Con l'ecografia bedside - anche gli apparecchi portatili di piccole dimensioni sono dotati di modulo color-Doppler - è possibile 'visualizzare' un rigurgito valvolare (insufficienza) e graduarne l'intensità così come è altrettanto possibile valutare l'entità di una stenosi (lieve, moderata, severa) sulla base della velocità di flusso trans-valvolare. L'indagine permette inoltre di valutare la presenza/assenza di versamento pericardico e se presente di valutarne l'entità evidenziando sia forme lieve di nessun significato fisiopatologico che forme severe come il tamponamento cardiaco: quest'ultimo se correttamente individuato rappresenta una condizione estremamente grave che impone un rapido trattamento (pericardiocentesi) utilizzando per l'indirizzo dell'ago la sonda ecografica. Oltre all'approccio diagnostico iniziale, l'ecografia bedside è uno strumento prezioso nel follow-up e nel monitoraggio della terapia: è infatti possibile rivalutare durante la degenza la funzione sistolica cosi come, nei pazienti con scompenso cardiaco, gestire la terapia diuretica monitorando la riduzione/scomparsa di un eventuale versamento pleurico presente all'ingresso e/o la ripresa della normale cinetica e riduzione del calibro della vena cava inferiore.

Anche se ostacolata da un generale scetticismo, soprattutto da parte dei medici dedicati all'imaging che ponevano dubbi sulla reale efficacia dell'indagine, l'ecografia polmonare si è imposta in questi ultimi anni e attualmente rappresenta un supporto prezioso nella valutazione pleuro-polmonare. Il riscontro di segni ultrasonografici predittivi, ormai validati da numerose evidenze scientifiche, ${ }^{11-15}$ ha modificato radicalmente l'approccio al paziente con patologia respiratoria. Alla semeiotica tradizionale (ispezione, palpazione, percussione e auscultazione) la bedside sonography aggiunge elementi utili sia nel chiarire la natura dei reperti semeiologici tradizionali che fornire informazioni sul grado di aerazione polmonare. Nel polmone normalmente aerato, a causa della elevata

Tabella 1. Rilievi ultrasonografici che permettono di confermare/escludere condizioni patologiche al letto del paziente internistico.

Ultrasonografia cardiaca

- $\quad$ È conservata la funzione (FE) del ventricolo sx?

- È dilatato il ventricolo sx?

- $\quad$ È presente ipertrofia del ventricolo sx?

- È presente una dilatazione atriale?

- È dilatato il ventricolo dx?

- È presente insufficenza (rigurgito) valvolare?

- È presente un versamento pericardico? C'è un tamponamento?

- La vena cava inferiore è dilatata (calibro $>25 \mathrm{~mm})$ ? Sono conservate, ridotte o assenti le variazioni con il respiro?

Ultrasonografia Vascolare

- È presente una stenosi carotidea?

- È presente un aneurisma dell'aorta addominale?

- $\quad$ E assente la CUS nelle vene degli arti inferiori (trombosi venosa profonda)?

Ultrasonografia Endocrina

- È ingrandita la ghiandola tiroidea?

- $\quad$ Ci sono noduli nel parenchima tiroideo? Se sì solidi o liquidi?

Ultrasonografia Polmonare

- È presente un versamento pleurico?

- $\quad$ È presente un pneumotorace (sliding assente)?

- Sono evidenti line B (code di cometa) in questo paziente dispnoico?

- È presente una consolidazione polmonare con broncogrammi aerei?

Ultrasonografia addominale

- È presente epatomegalia?

- È presente splenomegalia?

- $\quad \mathrm{Ci}$ sono calcoli in colecisti?

- $\quad$ È ispessita la parete colecistica (colecistite)?

- Le vie biliari sono dilatate in questo paziente itterico?

- È presente ascite?

- I reni sono ridotti di volume (grinzi)?

- Sono presenti calcoli urinari?

- È presente idro-nefrosi?

- È presente ritenzione urinaria?

- È aumentata di grandezza la prostata?

FE, frazione d'eiezione; CUS, ecografia per compressione. (Da F. Cipollini, 2015 It J Med; modificato). 
differenza di impedenza acustica tra l'aria contenuta nei polmoni e la parete toracica sarà visualizzabile solo la linea pleurica ma se al di sotto della pleura parietale vi è una raccolta liquida, ovvero un elemento facilmente attraversato dagli ultrasuoni, questa sarà visualizzata. L'ecografia polmonare rappresenta attualmente il gold standard nella diagnosi di versamento pleurico. In questa condizione l'indagine ha un'elevatissima sensibilità e specificità e permette di valutare correttamente il volume del versamento. La semeiotica tradizionale aveva proposto alcuni segni semeiologici fisici per definire l'entità del versamento pleurico; i medici più attempati ricorderanno sicuramente la linea di Damoiseau Ellis, il triangolo di Garland ed il triangolo opposto di Grocco. L'ecografia polmonare ha una elevatissima sensibilità nel definire rapidamente ed esattamente un versamento pleurico e valutarne sia il volume che le caratteristiche e permette inoltre di 'guidare' una toracentesi anche per versamenti di pochi millilitri. Altra indicazione nella quale l'ecografia rappresenta l'indagine diagnostica di riferimento è il pneumotorace. In questa condizione l'indagine ecografica in tempo reale non mette in evidenza come nel soggetto sano - il gliding (scivolamento) ovvero l'interfaccia ecogena della pleura viscerale che scorre su quella parietale; nel pneumotorace l'interfaccia pleurica resta immobile nonostante gli atti respiratori.

L'ecografia, sempre nella diagnostica polmonare, ha inoltre identificato un nuovo parametro che è risultato di notevole importanza nell'approccio al paziente con sintomi respiratori. ${ }^{12}$ Quando si riduce il grado di aerazione polmonare (per aumento della componente trasudatizia/essudatizia) si generano delle immagini artefattuali verticali, definiti convenzionalmente 'linee B' o 'code di cometa'. Questi artefatti verticali rappresentano un segno ecografico sensibile di acqua vascolare extrapolmonare ed il numero di queste lung comets tail correla sia con la classe NYHA dello scompenso cardiaco sia con i valori del NT proBNP. ${ }^{13,14}$ Proprio in base al numero - e quindi all'acqua extravascolare che imbibisce l'interstizio polmonare - l'aspetto ecografico può variare da un quadro normale c.d. black lung a quello di un black and white lung ovvero con line B in numero moderato, sino ad arrivare ad un withe lung come nell'edema polmonare acuto. ${ }^{13} \mathrm{Ma}$ con l'ecografia è possibile anche osservare un 'consolidamento polmonare' (come ad esempio polmonite, addensamento infiltrativo, vascolare) a condizione che lo stesso raggiunga il mantello pleurico - ovvero non vi sia interposizione di parenchima normale tra la pleura e il focolaio patologico oppure il consolidamento sia separato dalla parete toracica da falda di versamento pleurico che funge da finestra acustica. Con la massiva perdita di ventilazione alveolare ed il contestuale aumento di fluidi (essudato/trasudato) e/o infiltrato infiammatorio/necrotico l'assenza di aria negli spazi alveolari determina la scomparsa del tipico quadro artefattuale (A-lines). Quando l'area di consolidamento polmonare raggiunge il mantello pleurico (evenienza che si verifica nell' $80 / 90 \%$ dei casi) e non vi è interposizione di parenchima normale tra la sonda e il focolaio patologico, il fascio ultrasonoro è in grado di rappresentarlo come immagine reale. Aree di consolidamento che affiorano alla pleura viscerale, quindi evidenti all'ispezione ecografica, sono espressione sia di malattie infiammatorie come le polmoniti ma anche di malattie neoplastiche, granulomatose macronodulari, alveolitiche, infartuali e di atelectasia. Dai dati della letteratura è ormai dimostrato che l'ecografia è sicuramente non inferiore ed in alcuni studi addirittura superiore all'esame radiologico standard del torace e pertanto può rappresentare, oltre che una integrazione, una valida alternativa all'esame radiologico sia in emergenza che nelle degenze ordinarie. In un nostro lavoro ${ }^{15}$ l'ecografia bedside eseguito all'ingresso in pazienti molto anziani (nei quali una indagine rx standard del torace è problematica per la difficoltà o addirittura impossibilità di mantenere la stazione eretta, di mantenere apnea respiratoria ed eseguire l'indagine in due proiezioni) ha dimostrato una sensibilità superiore (seppur in maniera non significativa) rispetto all'indagine radiologica standard. Poter diagnosticare subito all'ingresso una polmonite permette di poter iniziare rapidamente una terapia antibiotica con $\mathrm{i}$ ben noti vantaggi sull'outcome della malattia (Figura 1).

Sulla base di questi dati è pertanto possibile un approccio semplice ed affidabile nella valutazione dei pazienti con insufficienza respiratoria acuta soprattutto nelle situazioni di urgenza/emergenza come si verifica nei Dipartimenti di Emergenza e nelle Medicine Interne (Figura 2). Proprio per la rapidità e il valore predittivo di alcuni segni ecografici la bedside sonogrphy ha sicuramente modificato l'approccio clinico del paziente con dispnea. Questa rappresenta un sintomo che può essere causato da diverse condizioni patologiche, alcuni delle quali possono presentarsi acutamente ed essere pericolose per la vita (ad es., embolia polmonare, infarto miocardico acuto). In queste situazioni la valutazione rapida e mirata è fondamentale per l'outcome del soggetto. Proprio per questo la valutazione diagnostica differenziale delle dispnee rappresenta una sfida per il medico. L'ecografia polmonare permette di aumentare ulteriormente la probabilità di diagnostica differenziale perché permette di rilevare segni indicativi di specifiche patologie. 
Nel paziente dispnoico l'immagine ecografica potrà mostrarci un reperto normale oppure un quadro riferibile a sindrome interstiziale con la presenza di linee $\mathrm{B}$ (code di comete ultrasoniche) in numero più $\mathrm{o}$ meno maggiore o un consolidamento polmonare. Sulla base di questi segni è pertanto possibile un approccio semplice ed affidabile nella valutazione dei pazienti con dispnea acuta soprattutto nelle situazioni di urgenza dove il tempo rappresenta un elemento prognostico decisivo. Il riscontro di linee-B permette di differenziare una dispnea cardiogena da una respiratoria. Infatti nelle esacerbazioni della broncopneumopatia cronica ostruttiva,nell' embolia polmonare, nella polmonite così come nel pneumotorace, non è mai visibile un pattern ecografico caratterizzato dalla presenza delle code di cometa ultrasoniche.D'altra parte nei pazienti con insufficienza cardiaca (e in numero elevato nell'edema

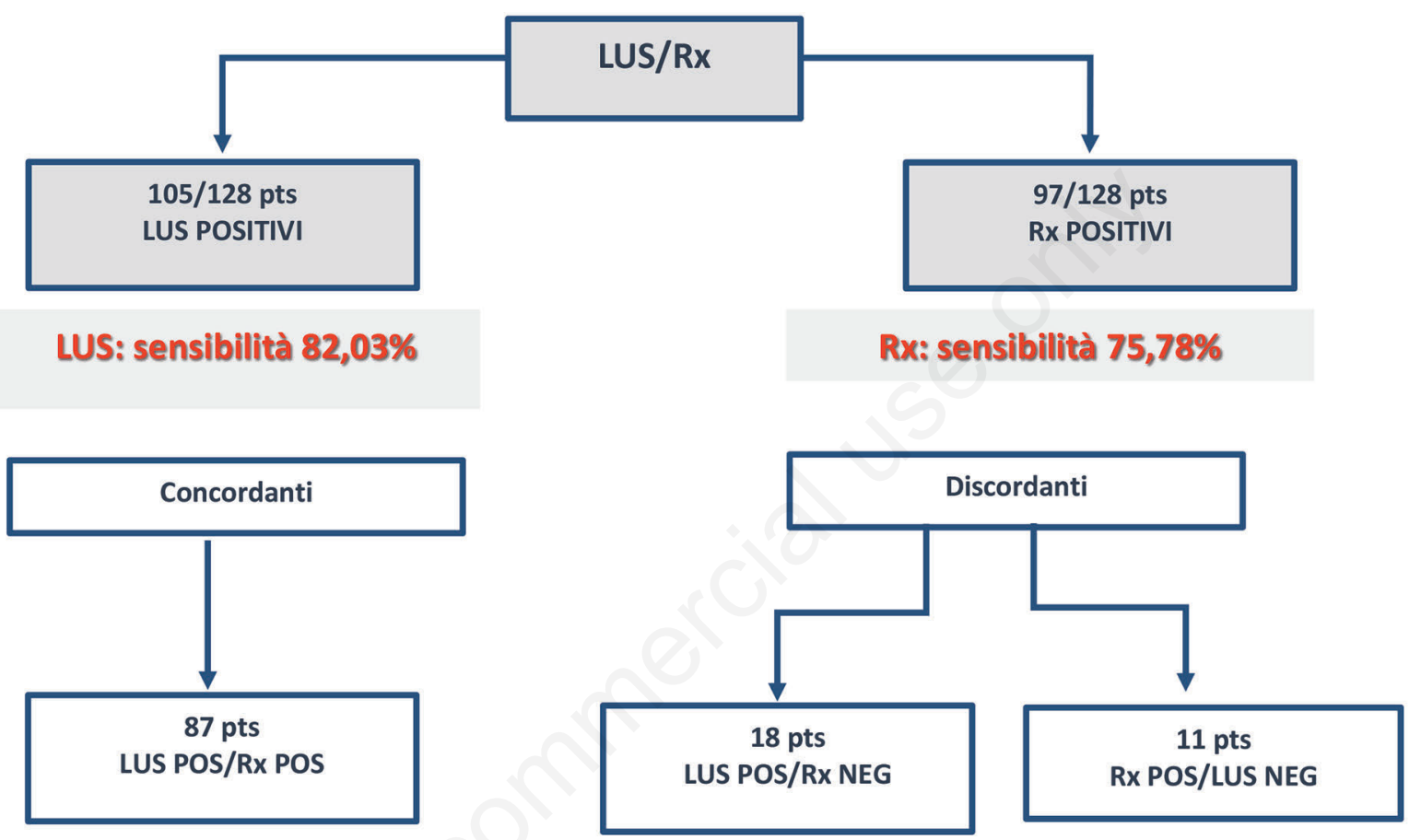

Figura 1. Confronto ecografia torace (LUS) vs Rx torace nella diagnosi di polmonite nel paziente molto anziano in Medicina Interna. (Da F. Cipollini et al. It J Med 2018;12:127-1931).
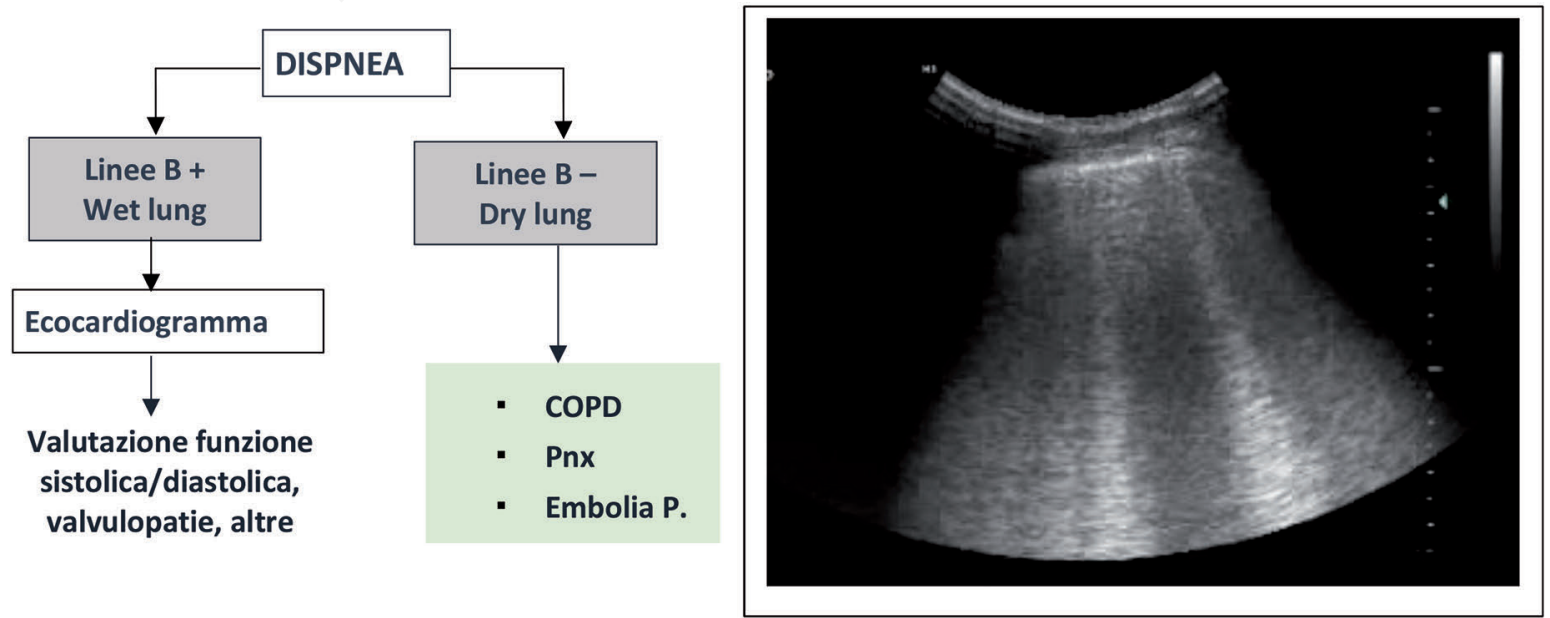

Figura 2. Approccio clinico - ecografico del paziente dispnoico. 
polmonare acuto) le linee B sono presenti e rappresentano un segno fedele di acqua polmonare extravascolare. Pertanto nel paziente dispnoico un esame ecografico polmonare permetterà di valutare sulla base della presenza o meno di code di cometa ultrasoniche - se ci troviamo di fronte ad un paziente con polmone umido o secco. Nel primo caso oltre ad avere un segno affidabile di 'congestione polmonare' sarà possibile iniziare rapidamente la terapia diuretica e monitorarla ripetendo l'indagine ad intervalli e programmare il successivo iter diagnostico con l'indagine ecocardiografica finalizzata alla valutazione della funzione cardiaca e della natura della cardiopatia. In caso di 'dry lung' l'iter diagnostico - e la conseguente terapia - sarà orientato alla valutazione morfo-funzionale respiratoria. ${ }^{16}$ Sempre a livello polmonare, nei pazienti con dispnea cardiogena, è possibile osservare e valutare la presenza o meno di versamento pleurico cosi come - spingendoci ancora più in basso a livello addominale - valutare il calibro (aumentato o meno) e la cinetica respiratoria ${ }^{17}$ della vena cava inferiore (ipo-acinesia o normocinesia) (Figura 3). La presenza di questi segni non solo potrà aggiungere elementi a conferma dell'insufficienza cardiaca ma fornirà al clinico importanti parametri di riferimento nel follow-up ecografico finalizzato alla valutazione dell'efficacia della terapia. Giova ricordare che in era pre-ecografica, nell'impossibilità di valutare direttamente la pressione atriale $\mathrm{dx}, \mathrm{i}$ metodi indiretti utilizzati erano esclusivamente clinici (distensione delle v. giugulari, reflusso epatogiugulare, tono aggiunto $\mathrm{S} 3$, ecc.) che comunque sono risultati non affidabili per la loro ridotta sensibilità $\mathrm{e}$ specificità. ${ }^{18}$ L'ecografia bedside offre un supporto anche nell'approccio diagnostico al paziente dispnoico sospetto per trombo-embolismo venoso. In questi pazienti oltre alla possibilità di visualizzare lesioni suggestive per lesione trombo-embolica, l'indagine può essere estesa anche a livello cardiaco (per la ricerca di segni di impegno ventricolare $\mathrm{dx}$ ) e venoso periferico eseguendo una ecografia per compressione (CUS) che ha una elevata sensibilità e specificità per la diagnosi di trombosi venosa profonda. Per aumentare la sensibilità diagnostica, oltre agli score clinici e al dosaggio del D-dimero, è stato proposto di eseguire un'indagine ecografica multi-distrettuale: polmonare, cardiaca e venosa agli arti inferiori. Un tale approccio ecografico multi-organo ${ }^{19}$ ha dimostrato di incrementare la sensibilità $(90 \%)$ in percentuale significativamente superiore rispetto a ciascuna delle singole metodiche (polmone, cuore, arti inferiori). $\mathrm{Ma}$ il dato più significativo riguarda l'elevatissimo valore predittivo negativo per embolia polmonare $(95 \%)$ che è risultato dall'impiego della indagine ecografica polidistrettuale: questo dato è estremamente importante in quanto, associato con il dosaggio del D-dimero e con gli scores clinici, la bedside sonography trova indicazione nei pazienti con sospetta embolia polmonare come indagine preliminare prima di essere sottoposti ad angio-TC.

L'integrazione ecografica ha notevolmente modificato anche la valutazione dell'apparato circolatorio. La semeiotica tradizionale permette con la palpazione di valutare la presenza/assenza dei polsi periferici arteriosi nei punti di repere definiti e con l'ascoltazione permette di rilevare la presenza di soffi (soprattutto a livello carotideo). L'ecografia oltre a definire la pervietà o meno delle arterie periferiche degli arti,permette di visualizzare la presenza di apposizioni trombotiche, di stenosi e di valutarne l'entità. Permette inoltre la valutazione dei vasi intraddominali ed in particolare di indagare se vi sia o

\begin{tabular}{|l|l|l|}
\hline $\begin{array}{l}\text { Calibro vena } \\
\text { cava inferiore }\end{array}$ & $\begin{array}{l}\text { Variazioni } \\
\text { respiratorie }\end{array}$ & $\begin{array}{l}\text { Pressione atriale } \\
\text { dx (mmHg) } \\
\text { stimata }\end{array}$ \\
\hline $\begin{array}{l}<1,5 \mathrm{~cm} \mathrm{o} \\
\text { meno }\end{array}$ & Collasso & $0-5$ \\
\hline $1,5-2,5 \mathrm{~cm}$ & Riduzione $>50 \%$ & $5-10$ \\
\hline$>2,5 \mathrm{~cm}$ & Riduzione $<50 \%$ & $10-15$ \\
\hline$>2,5$ dilated & Riduzione $<50 \%$ & $15-20$ \\
\hline $\begin{array}{l}\text { Dilatazione } \\
\text { vene epatiche }\end{array}$ & Acinesia & $>20$ \\
\hline
\end{tabular}

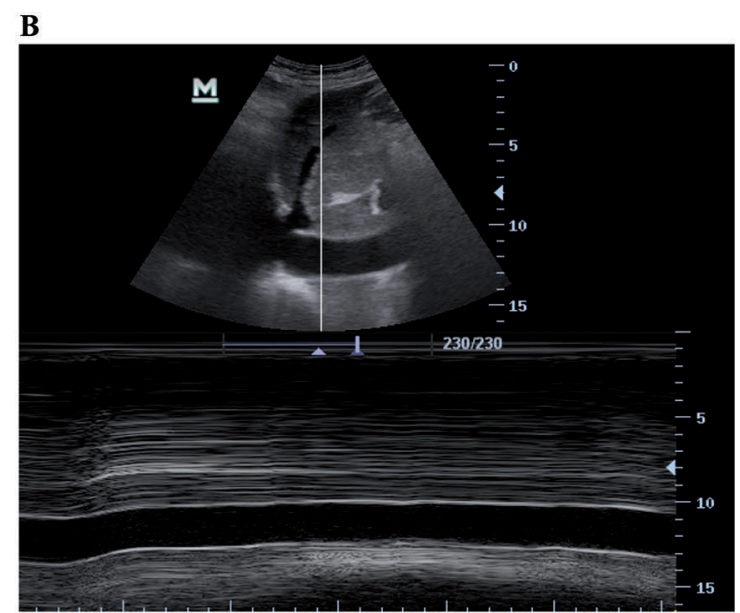

Figura 3. A) Correlazione tra calibro vena cava inferiore, variazioni respiratorie e pressione atriale destra; B) Vena cava inferiore dilatata e acinetica in paziente con scompenso cardiaco. 
meno un aneurisma dell'aorta addominale (patologia questa sempre più frequente da osservare in ambiente internistico). Nel distretto carotideo in particolare, l'indagine permette di valutare,qualora presente, non solo la presenza di una stenosi ma di definirne il grado oltre ad avere informazioni sulle caratteristiche della placca stenosante: elementi questi estremamente importanti per definire l'iter diagnostico-terapeutico più appropriato. Sul versante venoso l'ecografia bedside permette di valutare in modo molto semplice ed efficace la pervietà dei segmenti venosi degli arti (soprattutto inferiori) per mezzo della ecografia per compressione (CUS). Questa indagine, fruibile al letto del malato, è estremamente sensibile e specifica per definire la presenza/assenza di trombosi venosa semplicemente esercitando una compressione con la sonda ecografica sul vaso e rilevandone la comprimibilità (vaso normale) o la non comprimibilità (nella trombosi venosa).

Tuttavia, se vi è un settore dove l'integrazione ecografica ha rivoluzionato l'approccio clinico al paziente e permesso di indirizzare correttamente l'iter diagnostico, questi è l'addome. In era pre-ecografica erano veramente pochi i segni fisici indicativi: l'ecografia ha apportato elementi in grado o di definire specifiche alterazioni patologiche oppure di indirizzare correttamente il clinico alle indagini laboratoristico-strumentali di secondo livello. Permette innanzitutto di definire correttamente le dimensioni degli organi ipocondriaci soprattutto in quelle particolare condizioni di varianti morfologiche nei quali i punti di repere classici non sono più di riferimento. Consente di valutare le reali dimensioni del fegato, $i$ contorni, la vascolarizzazione, l'eventuale presenza di lesioni e - qualora presenti - permette di definirne correttamente la natura (solida/liquida). Analoghi rilievi la scansione permette di rilevare a carico della milza in particolare consente di valutare correttamente la volumetria dell'organo molto spesso difficile da valutare se non nelle splenomegalie di un certo grado. Permette di valutare la ghiandola pancreatica di definirne le dimensioni, l'ecostruttura, l'eventuale dilatazione del dotto di Wirsung e, se presente, la presenza di lesioni focali (solide/liquide) endo-perighiandolari. L'ecografia ha permesso inoltre di visualizzare la colecisti e i rami principali delle vie biliari (epatico,coledoco) modificando radicalmente l'approccio alla patologia biliare. La semeiotica tradizionale indicava nella palpazione dolente del punto cistico e nella manovra di Murphy i segni semeiologici palpatori indicativi di patologia biliare. L'ecografia bedside permette di visualizzare la colecisti direttamente, valutarne lo spessore di parete e l'eventuale presenza di calcoli anche di piccole dimensioni. Analogamente riesce a visualizzare le vie biliari extra-epatiche, l'eventuale dilatazione delle stesse e le patologie contestuali. Nel soggetto normale le vie biliari intra-epatiche (tranne i segmenti principali lobari) non sono visualizzabili: in caso di ostruzione biliare l'ecografia permetterà di visualizzare anche le diramazioni più periferiche $\mathrm{e}$ definire la sede dell'ostruzione in base ai rami ectasici. Proprio grazie a questi segni sonografici estremamente predittivi, è profondamente cambiato l'approccio clinico al paziente con ittero. Tralasciando la diagnostica differenziale del secolo scorso che indicava come segni differenziali 'la sfumatura' della colorazione itterica (qualcuno più attempato ricorderà l'ittero rubinico che avrebbe dovuto orientare a una forma epato-cellulare da uno verdinico che invece avrebbe indirizzato verso un ittero ostruttivo), la diagnostica degli itteri in era pre-ecografica poneva le basi sulle frazioni della bilirubinemia (diretta $v s$ indiretta). Questi parametri non avevano un valore predittivo adeguato per l'elevato overlap di valori nelle due condizioni (ostruttivo vs epato-cellulare). Con l'impiego della ecografia bedside la diagnosi differenziale ${ }^{20}$ degli itteri è già possibile all'ingresso del malato senza perdite di tempo. L'indagine potrà mostrarci vie biliari normali (ovvero non dilatate) ed in questo caso ci orienteremo verso un ittero c.d. medico prescrivendo le indagini siero-ematologiche del caso. Oppure saranno visualizzate (dilatate!) le vie biliari intra-epatiche ed allora ci orienteremo verso una forma ostruttiva e prescriveremo le indagini strumentali specifiche (ERCP, colangioRM).

Ancora maggiore è il contributo dell'ecografia bedside nel paziente con dolore addominale. Questa condizione rappresenta una sfida per il medico per il fatto che la semeiotica tradizionale offre pochi elementi di giudizio nell'inquadramento del paziente con dolore addominale acuto: non a caso i vecchi clinici descrivevano l'addome come 'la tomba del medico'. Nelle casistiche più favorevoli l'esame clinico riesce a identificare/orientare la diagnosi in non più del $50-55 \%$ dei casi; percentuale che si riduce ulteriormente intorno al 30\% nel paziente anziano in quanto l'esame clinico è ostacolato dalla difficoltà di comunicazione, raccolta dell'anamnesi e deficit sensoriali (ipoacusia, demenza). Anche esami ancillari (amilasi/conta leucociti) non hanno di molto modificato questa percentuale:la specificità ed il valore predittivo di questi esami sono infatti inferiori al $50 \%$ e pertanto non adeguati ad un discrimine diagnostico. Analogamente le indagini radiologiche dirette (rx addome senza contrasto) non hanno incrementato significativamente la natura della sintomatologia addominale: i segni di perforazione e occlusione sono infatti presenti solo nel $50-60 \%$ dei casi. $^{21,22}$ L'ecografia bedside, invece,ha permesso di incrementare questa percentuale potendo sia identificare direttamente la causa del dolore 
addominale (es. litiasi colecistica, litiasi renale) sia indirizzare l'iter diagnostico verso altre cause (addominale o extra-addominale). Alcuni esempi: in un soggetto con dolore epigastrico l'esame può evidenziare la presenza di litiasi biliare, un aumento del volume della ghiandola pancreatica sospetta per flogosi acuta. Se il dolore è localizzato al quadrante superiore $\mathrm{dx}$ può mostrare,oltre ad una litiasi,un ispessimento della parete della vescichetta biliare suggestiva per colecistite. A carico dei quadranti inferiori può mettere in evidenza un ispessimento delle pareti ileo-coliche suggestive per una m. di Crohn, la presenza di raccolte peri-coliche suggestive per diverticolite, oppure una calcolosi pielo-ureterale, ecc. Naturalmente la rapida identificazione della patologia responsabile del dolore addominale acuto permette di instaurare altrettanto rapidamente la terapia specifica (medica o chirurgica ) perché giova ricordare che in questi pazienti - soprattutto se anziani con comorbidità - diversamente da altre situazioni cliniche non ci è concessa la possibilità di prolungare l'osservazione 0 ricorrere ad indagini particolari: in questi casi è importante prendere una decisione ed agire.

L'ecografia bedside permette inoltre di valutare l'apparato linfatico.Oltre a definire correttamente le dimensioni della milza - e pertanto identificare condizioni di spleno-megalia moderata - l'esame permette di evidenziare, se presenti, alterazioni focali dell'ecostruttura o lesioni traumatiche: le fratture spleniche sono infatti riconoscibili quando ancora la capsula splenica è integra. La lesione può così essere monitorata e nella eventualità che si verifichi la lacerazione della capsula (la c.d. frattura in due tempi) e trattata tempestivamente. L'ecografia permette inoltre di valutare se presenti linfo-adenomegalie endo-addominali, prevalentemente in sede periaortica, non apprezzabili con l'esame clinico. Anche i linfonodi superficiali, peraltro rilevabili con la semeiotica tradizionale che permette di valutarne le dimensioni e la consistenza, si giovano dell'integrazione ecografica in quanto l'indagine permette in base a specifiche caratteristiche, quali il roundness index e la presenza/assenza dell'ilo, di differenziare con notevole attendibilità una linfoadenopatia di natura reattiva da una di sospetta natura infiltrativa.

Anche allo studio del sistema endocrino l'ecografia bedside ha dato un discreto contributo. Sicuramente la ghiandola tiroidea è apprezzabile con l'ispezione e la palpazione manuale che permette di valutare la consistenza e l'eventuale presenza di formazioni nodulari. Con l'ecografia, peraltro, è possibile definire correttamente le dimensioni della ghiandola sia nelle singole porzioni dell'organo che in toto. Permette inoltre di valutare la struttura ecografica (elemento di notevole aiuto diagnostico nella patologia infiammatoria acuta, sub-acuta e cronica) e definire la natura dell'eventuale presenza di alterazioni focali. Permette in particolare di definire se un nodulo è liquido (cistico) o solido ed in quest'ultimo caso permette di cogliere alcuni elementi (micro-calcificazioni, larghezza, contorni, ecc.) che orientano correttamente il successivo iter diagnostico e soprattutto riducono il numero (a volte inappropriato) delle cito-aspirazioni/biopsie eseguite per la tipizzazione cito-istologica dei noduli stessi. L'ecografia ha permesso inoltre di visualizzare alcune ghiandole endocrine come le surrenali non rilevabili con la semeiotica tradizionale. L'ecografia permette di definirne le dimensioni, la mono o la bilateralità e le eventuali lesioni focali. Va tuttavia segnalato che la diffusione dell'indagine ecografica ha incrementato i c.d. incidentalomi ovvero aumenti volumetrici delle ghiandole surrenali in assenza di patologie clinicamente o laboratoristicamente evidenti.

Da ultimo ma non per ultimo giova ricordare come l'ecografia abbia modificato sostanzialmente l'esecuzione di procedure diagnostiche invasive al letto del malato. Per quanto concerne la toracentesi, la semeiotica tradizionale raccomandava di inserire l'ago nella linea medio-scapolare sul margine superiore della costa a livello di uno-due spazi intercostali al di sotto la sommità del versamento e quindi continuare a far penetrare l'ago fino a che non si aspirava il liquido pleurico. Con la toracentesi eco-assistita o ancor meglio eco-guidata è possibile identificare lo spazio e la posizione più favorevole per eseguire la puntura senza rischi per il paziente e con una probabilità di successo pressoché del 100\%. Non solo. La guida ecografica permette di eseguire la toracentesi anche in caso di versamenti di minima entità ed in quelli saccati. Stesso discorso vale per la paracentesi: la semeiotica tradizionale indicava di eseguire la puntura a livello del terzo laterale della linea ombelico-spina iliaca antero-superiore. Con l'assistenza o ancor meglio con la guida ecografica è possibile anche a livello dell'addome eseguire una paracentesi anche in caso di raccolte di piccole quantità con percentuali di successo anche a questo livello pressoché del $100 \%$ e senza rischi per il paziente. La guida ecografica, oltre per le punture esplorative sopra elencate, è utilizzata per le biopsie dei parenchimi addominali, per la rachicentesi, per il controllo del corretto posizionamento del catetere vescicale e del sondino naso-gastrico. Negli ultimi anni vi è stato inoltre un notevole sviluppo dell'utilizzo della guida ecografica per il cannulamento di vene periferiche ed il posizionamento di PICC. La guida ecografica ha inoltre pressoché completamente sostituito la metodica della 'puntura alla cieca' della vena succlavia o giugulare per il posizionamento di catetere venoso centrale riducendo 
in modo drastico l'incidenza del pneumotorace postprocedurale.

\section{Key point}

La bedside sonography (o Point of Care Ultrasound) eseguita direttamente dal clinico (in particolare l'internista) e non dal medico dedicato all'imaging, orienta l'indagine al sintomo e non all'organo realizzando più rapido ed appropriato l'iter diagnostico-terapeutico.

\section{Le prospettive della semeiotica clinico-strumentale del futuro}

Quali ulteriori sviluppi avrà la semeiotica strumentale al letto del malato nei prossimi anni? 'Il futuro è adesso' afferma la pubblicità di una nota casa automobilistica; questa affermazione può essere applicata alla medicina attuale. Infatti probabilmente - o forse sicuramente - non dovremmo attendere altri duecento anni prima che una nuova scoperta fruibile al letto del malato permetta al medico di rivoluzionare e migliorare l'approccio diagnostico cosi come è stato per lo stetoscopio nel XVIII e per l'ultrasonografia nel $\mathrm{XX}$ secolo. Il bagaglio strumentale del clinico già oggi è dotato sia di strumenti tradizionali (fonendoscpopio e sfigmomanometro), di elettrocardiografi sempre più miniaturizzati e performanti, di ossi-saturimetro, di ecografo hand-held o portatile e sia inoltre di un laptop/tablet su cui poter accedere ad una serie di applicazioni in grado di supportare in tempo reale il medico su parametri di laboratorio, dosaggi terapeutici, interazioni farmacologiche, linee guida, ecc. Questi nuovi strumenti tecnologici e le loro applicazioni modificheranno l'approccio al malato? È auspicabile ma allo stato non possiamo affermarlo. ${ }^{23}$ Possiamo anzi dobbiamo porci invece una domanda: il medico è formato adeguatamente per un corretto utilizzo di queste nuove tecnologie finalizzate ad un reale miglioramento della diagnosi e della cura del malato? Allo stato, è possibile fare una riflessione critica - sulla base di dati consolidati in letteratura sull'utilizzo della bedside sonopraphy ovvero la tecnologia che ha maggiormente impattato la clinica nell'ultimo decennio e per la quale la formazione e l'addestramento sono essenziali per un reale miglioramento della performance clinica al letto del malato. Da un lato ci sono diversi clinici sempre più numerosi che propongono l'uso diffuso di ecografi portatili, motivando questa affermazione con il fatto che la bedside sonography ha completamente cambiato la loro pratica clinica quotidiana in quanto strumento utile, che permette una diagnosi tempestiva ed il conseguente idoneo trattamento. Vi è però dall'altro lato il giustificato timore (soprattutto a garanzia del paziente) che a fronte di questa diffusione l'impiego degli ultrasuoni da parte del clinico non si coniughi con una adeguata formazione ed addestramento: come dicono alcuni referenti di società scientifiche dedicate all'imaging l'ecografo deve essere usato da chi è stato addestrato ad usarlo. Il problema della POCUS sta nel fatto che è una metodica operatore dipendente: le immagini ottenute con una macchina ad ultrasuoni portatile di solito non vengono memorizzate oppure ne vengono registrate solo alcune (diversamente dalle indagini radiologiche che sono completamente archiviate e pertanto rivalutabili anche con un second-look). Nel caso dell'ecografia bedside è il clinico che esegue l'indagine, che sceglie quali e quante scansioni eseguire, le interpreta in tempo reale e pertanto le conclusioni diagnostiche dipendono dalle sue conoscenze e dalla sua esperienza. Un training non adeguato o insufficiente espone la procedura a rischi per il paziente di diagnosi false positive che generano procedure o test aggiuntivi o false negative che non individuano una patologia potenzialmente grave. La sicurezza dei pazienti dovrebbe essere centrale e le autorità sanitarie dovrebbero garantire che gli utilizzatori di ecografi siano adeguatamente formati e che ciò sia mantenuto attraverso adeguati sistemi di audit a garanzia della qualità della prestazione. Sebbene l'ultrasonografia bedside sia uno strumento clinicamente utile con applicazioni pertinenti nella maggior parte delle specialità, i livelli di formazione necessari per garantire un uso accurato non è stato ancora definito. Per questo motivo è necessaria introdurre la formazione ecografica all'interno del programma educativo dei medici (in particolare in quelli post-laurea) ad integrazione dei curricula già esistenti perché, nonostante le prove a sostegno della validità dell'indagine eseguita dal medico, una strategia educativa e linee guida ampiamente accettate per le competenze ultrasonografiche bedside devono ancora essere stabilite.

Per raggiungere questo obiettivo è necessario implementare modelli formativi teorico-pratici che garantiscano l'acquisizione di competenze adeguate per l'esecuzione dell'ecografia. E a questo riguardo si pone un quesito: come si definisce la competenza con questa tecnologia emergente ed in continua espansione? Man mano che si acquisiscono maggiori elementi sulle possibilità e i limiti della metodica, è fondamentale sviluppare una metodologia validata per definire e misurare le competenze tra i medici in formazione. In linea generale, la valutazione delle competenze dovrebbe includere: i) tests che verifichino l'acquisizione e l'applicazione di conoscenze relative al POCUS, ii) la dimostrazione delle competenze tecniche (ad esempio, una corretta selezione della sonda, il posizionamento e l'ottimizzazione delle 
immagini); iii) un'efficace integrazione nella pratica clinica di routine. Queste valutazioni possono essere effettuate per mezzo di diverse metodologie tra cui applicazioni web-based, simulatori, pazienti standardizzati e casi clinici reali. ${ }^{24}$ L'Alliance of Academic Internal Medicine ha pubblicato sull'American Journal of Medicine ${ }^{25}$ un position statement avente finalità di supporto e fornendo potenziali metodi per implementare modelli di valutazione della competenza ultrasonografica bedside.Attualmente sono proposti diversi modelli formativi che vanno dal corso intensivo breve ai programmi di formazione che prevedono integrazioni longitudinali (naturalmente preferibili a quelli spot). Il modello dovrebbe fornire delle competenze che permettano: i) di avere le conoscenze riguardanti l'indicazione e i limiti dell'indagine; ii) conoscere il quesito clinico riguardante il caso in esame per restringere il campo della diagnosi differenziale o guidare una procedura; iii) avere l'abilità di acquisire le immagini a ultrasuoni; e iv) di interpretare le immagini acquisite ed utilizzarle per indirizzare ed ottimizzare il processo decisionale diagnostico terapeutico del paziente in esame. Naturalmente il percorso formativo deve prevedere momenti di didattica con adeguati periodi di formazione sul campo.

Ma a che punto è allo stato la formazione ecografica nei corsi di laurea in Medicina e Chirurgia e nelle Scuole di Specializzazione Mediche? Durante il Corso di Laurea vi sono attualmente corsi sperimentali finalizzati alla migliore comprensione dell'anatomia attraverso l'indagine ultrasonografica: si tratta di progetti limitati ad alcune realtà ed ancora non standardizzate. Per quanto riguarda la formazione ecografica nelle specialità mediche ed in particolare per la specializzazione in medicina interna, a parte poche eccezioni, non vi sono, durante il corso post-laurea, programmi specifici, valutazione delle competenze e standardizzazione della qualità della formazione mirata alla diagnostica ultrasonografica. La Federazione delle Associazioni Dirigenti Ospedalieri Internisti (FADOI) ha cercato di colmare questa lacuna implementando e organizzando la Scuola di Ultrasonografia in Medicina Interna diretta a sviluppare la competenza degli internisti ospedalieri in Ecografia Internistica. Nel suo documento di Clinical Competence dell'Internista Ospedaliero $^{26}$ la Scuola di Ecografia Internistica della Società Scientifica ha identificato tre livelli di competenza - con le relative skills richieste - nella diagnosi ecografica: di base, intermedia e avanzata. Il livello di base è costituito da un corso teorico di $32 \mathrm{~h}$ seguito da una parte pratica di $80 \mathrm{~h}$ che consentirebbe all'internista ospedaliero di identificare gli usi appropriati dello strumento, acquisire immagini ecografiche in tempo reale, interpretare i risultati ecografici e utilizzare gli ultrasuoni nel processo decisionale clinico. Nei prossimi anni gli organi competenti delle scuole di specialità mediche ed in particolare della medicina interna, dovranno definire i livelli di competenza ed integrare adeguatamente la formazione ecografica all'interno dei programmi formativi. Quantunque siano di piccole dimensioni, gli strumenti ecografici attualmente disponibili sono altamente performanti se usati correttamente e a costi ragionevoli, e pertanto è atteso negli anni futuri un incremento del numero di clinici che utilizzano l'indagine. Attualmente infatti non sembrerebbero molti gli utilizzatori della metodica: in una survey eseguita dalla FADOI nel $2011^{27}$ su un campione di 1712 medici internisti ospedalieri, solo $402(23,5 \%)$ praticavano ecografia bedside. È questa la vera sfida del prossimo futuro ovvero la diffusione capillare dell'ecografia bedside da parte di clinici adeguatamente formati. È sicuramente un progetto ambizioso, non è semplice e richiederà tempo, tuttavia il percorso è obbligato se si vuole ottenere dall'esame clinico-ecografico integrato un reale miglioramento in termini di efficacia nell'approccio clinico al letto del malato.

Scott D. Solomon ${ }^{28}$ in un commento pubblicato sul New England Journal of Medicine scriveva: A generation of physicians will need to be trained to view this technology as an extension of their senses, just as many generations have viewed the stethoscope. That development will require the medical education community to embrace and incorporate the technology throughout the curriculum. Oltre che un auspicio è un invito rivolto a tutti coloro che si occupano di formazione (Università in primis ma anche le Società Scientifiche Mediche): se la diagnostica ultrasonografica entrerà nei programmi formativi dei futuri medici, l'ecografia bedside avrà una capillare diffusione e sarà lo strumento medico universalmente utilizzato dalla classe medica cosi come lo è stato lo stetoscopio di Laennec.

\section{Key point}

L'ecografia bedside è indagine operatore dipendente e necessita da parte di chi la esegue (l'internista in primis) di una adeguata formazione che purtroppo, tranne rare eccezioni, non viene fornita durante i corsi di specializzazioni mediche. In questo ultimo decennio sono state le Società Scientifiche tra cui la FADOI, a proporre l'addestramento alla metodica con corsi teorico e formazione sul campo.

\section{Conclusioni}

Dopo la scoperta dello stetoscopio, l'ecografo rappresenta sicuramente l'innovazione più importante per l'approccio diagnostico al letto del malato. Negli ultimi decenni l'evoluzione tecnologica delle 
apparecchiature ha reso disponibili apparecchi di piccole dimensioni (portatili o addirittura tascabili) ma ad elevata tecnologia ed a costi accettabili: ciò ha permesso l'incremento progressivo dell'utilizzo della metodica da parte dei clinici. L'ecografia con apparecchi portatili (o hand-helded) al letto del malato, integrando la semeiotica fisico-strumentale tradizionale, contribuisce a migliorare l'approccio al paziente realizzando quello che viene definito l'esame clinico-ecografico integrato. Questa integrazione è essenziale soprattutto nel paziente anziano (tipologia prevalente nei reparti di medicina in terna) a causa della difficoltà in questo setting di pazienti di eseguire correttamente una raccolta anamnestica ed un esame obiettivo completo. La bedside sonography (o Point of Care Ultrasound) eseguita direttamente dal clinico (in particolare l'internista) e non dal medico dedicato all'imaging, orienta l'indagine al sintomo e non all'organo realizzando più rapido ed appropriato l'iter diagnostico-terapeutico. La bedside sonography,come la diagnostica ecografica in generale, è indagine operatore dipendente e pertanto necessita da parte degli operatori clinici (internisti in primis) di una adeguata formazione che purtroppo sino ad ora, tranne rare eccezioni, non viene fornita durante il Corso di Laurea e nelle Scuole di Specialità.

\section{Bibliografia}

1. Auenbrugger L. Inventum Novum Ex Percussione Thoracis Humani ut Signo, Vindobonae Typis Joannis Thomae Trattner, 176

2. Laennec Renè THR. De l'Auscultation médiate ou traité du diagnostic des maladies des poumons et du cœur. Paris: J.A. Brosson et J.S. Chaude; 1819.

3. Wunderlich KRA. Das Verhalten der Eigenwarme in Krankheiten. Leipzig: Otto Wigard, 1868.

4. Hermann von Helmhlotz: Handbuch der physiologischen Optik, Leipzig 1856.

5. Riva Rocci S. Un nuovo sfigmomanometro, Gazzetta Medica di Torino, $1896 n^{\circ}$ 50-51.

6. Dussik K. 1942: Weitere ergebnisse der utraschalluntersuchung bei gehirnerkrankungen. in Acta Neurochirurgica 1942; 379-401.

7. Wild JJ. Use of high frequency waves for detecting changes of texture in living tissues; Lancet 1951; 655:7.

8. Yudkowsky R, Otaki J, Lowenstein T, et al. A hypothesis-driven physical examination learning and assessment procedure for medical students: initial validity evidence, Med Educ 2009, 43: 729-740.

9. Moore CL, Copel JA. Point-of-care ultrasonography. N Engl J Med 2011;364:749-57.

10. Cipollini F. Cipollini ML. A critical evaluation in the delivery of the ultrasound practice: the point of view of the Internal Medicine hospitalist Italian Journal of Medicine 2015; 9:522.
11. Lichtenstein DA, Meziere GA. Relevance of lung ultrasound in the diagnosis of acute respiratory failure: the BLUE protocol. Chest 2008;134:117-125.

12. Volpicelli G. Caramello V. Cardinale L, et al. Bedside ultrasound of the lung for the monitoring of acute decompensated heart failure. Am J Emerg Med 2008; 26:585-591.

13. Frassi F, Gargani L, Gligorova S, et al. Clinical and echocardiographic determinants of ultrasound lung comets. Eur J Echocardiogr. 2007;8(6):474-479.

14. Gargani L. Lung ultrasound: a new tool for the cardiologist. Cardiovasc Ultrasound. 2011;27(9):6.

15. Cipollini F, Mirauta MC. Bedside Lung Ultrasound in the Diagnosis of Pneumonia in Very Old Patients . It J Med; 2018;12: 127-1931.

16. Copetti R, Soldati G, Copetti P. Chest sonography: a useful tool to differentiate acute cardiogenic pulmonary edema from acute respiratory distress syndrome. Cardiovase Ultrasound 2008;6:16.

17. Lloyd TR. Accuracy of Central Venous Pressure Measurement From the Abdominal Inferior Vena Cava. Pediatrics 1992; 89: 506-508.

18. Stevenson LW, Perloff JK. The limited reliability of physical signs for estimating hemodynamics in chronic heart failure . JAMA 1989;261:884-8.

19. Nazerian P, Vanni S, Volpicelli G, et al. Accuracy of point-of-care multiorgan ultrasonography for the diagnosis of pulmonary embolism. Chest 2014;145: 950-7.

20. Cooper D, Tarrant J, Whelan G, et al. Ultrasound in the diagnosis of jaundice--a review. Med J Aust 1985; 143:381-5.

21. Stapakis JC. Diagnosis of pneumoperitoneum: abdominal CT vs. upright chest film. J Comput Assist Tomogr 1992;16:713.

22. Maglinte DD. The role of radiography in the diagnosis of small-bowel obstruction. Am J Roentgenol 1997; 168:1171.

23. Wittenberg M. Will ultrasound scanners replace the stethoscope? Brit Med J 2014; 348:3463.

24. Kumar A, Kugler J, Jensen T. Evaluation of Trainee Competency with Point-of-Care Ultrasonography (POCUS): a Conceptual Framework and Review of Existing Assessments. J Gen Intern Med 2019; DOI: 10.1007/s11606-019-04945-4.

25. LoPresti CM, Jensen TP. Dversdal R.K et al. Point of Care Ultrasound for Internal Medicine Residency Training: A Position Statement from the Alliance of Academic Internal Medicine, The American Journal of Medicine (2019), https://doi.org/10.1016/j.amjmed. 2019.07.019

26. Nardi R, Mathieu G, Berti F, et al. Evaluation models and items of clinical competence for the hospital physicians in internal medicine. Ital J Med 2011;5 (3S1):1-84

27. Cipollini F. Relazione $I^{\circ}$ Corso Ecografia Internistica FADOI 2011 (dati non pubblicati).

28. Solomon SD, Saldana F. Point of care ultrasound in medical education - Stop listening and look. N Engl J Med 2014;370:1083-108. 


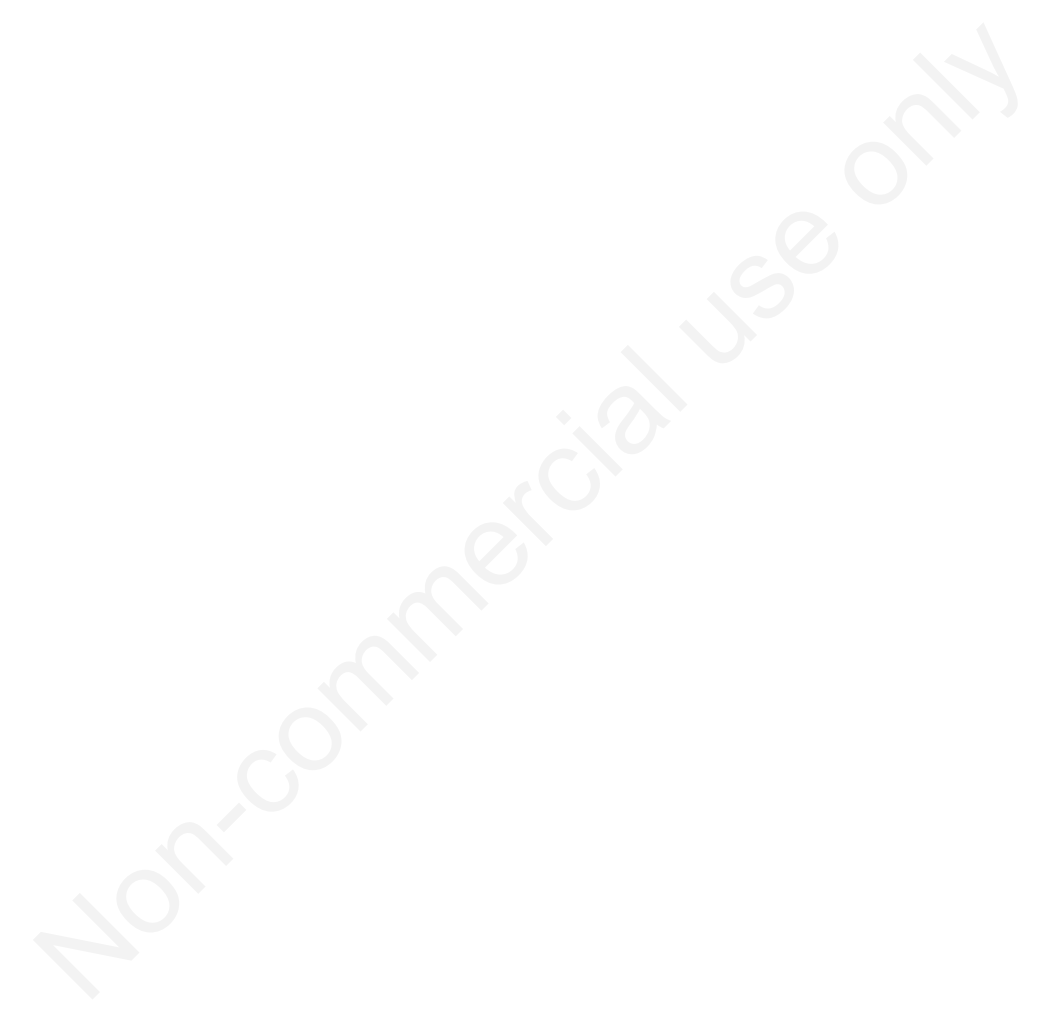




\section{LINEE GUIDA PER GLI AUTORI}

I Quaderni dell 'Italian Journal of Medicine (Quaderni ITJM), costituiscono una collana supplementare solo online annessa alla rivista Italian Journal of Medicine contenente lavori solo in lingua italiana.

I Quaderni ITJM pubblicano:

- Monografie ad hoc individuate dal Presidente FADOI, dal Consiglio Direttivo, dal Board Scientifico o dall'Editor in Chief dell'Italian Journal of Medicine, in funzione del contesto scientifico-istituzionale attuale.

- Monografie ad hoc su temi di particolare rilevanza scientifica a cura della Commissione FADOI Giovani.

- Traduzioni in italiano di alcuni lavori pubblicati sui numeri standard dell'Italian Journal of Medicine, di particolare interesse per la comunità scientifica.

\section{STESURA DEI LAVORI}

I lavori dovranno essere redatti in modo conforme alle linee guida sotto riportate:

- I manoscritti devono essere scritti interamente in lingua italiana, su documento di Word, con carattere Times New Roman/Arial, dimensione 12, formato A4, interlinea doppia e margini $2,54 \mathrm{~cm}$

Parole totali: $\max$ 4000; Sommario/Abstract: $\max 250$ parole; Bibliografia: min 40 voci; Tabelle e Figure: $3 / 5$ totali (le tabelle non devono superare n. 1 pagina del documento in Word).

- La strutturazione del contenuto deve attenersi agli standard internazionali per la Rassegna (Review): i) Abstract riassuntivo dell'intero lavoro; ii) Introduzione al tema trattato; iii) Criteri e strumenti di ricerca (criteri di inclusione/esclusione, banche dati consultate, ...); iv) i successivi paragrafi devono illustrare le più recenti scoperte scientifiche nel settore; v) Conclusioni; vi) Bibliografia.

- La prima pagina deve riportare: i) titolo (in stampatello minuscolo), senza acronimi; ii) nome e cognome per esteso di ciascun autore; iii) affiliazione(i) di ciascun autore, numerate con numeri arabi iv) eventuali ringraziamenti; v) nome e indirizzo postale completi dell'autore corrispondente, corredati da telefono, fax, e-mail; vi) da 3 a 5 parole chiave, separate da virgola. La seconda pagina può riportare: i) contributi degli autori, e.g. informazioni relative a contributi sostanziali delle persone coinvolte nello studio (http://www.icmje.org/\#author); ii) dichiarazione relativa a potenziali conflitti d'interesse; iii) ulteriori informazioni (e.g. fondi, esposizioni durante conferenze...).

- In caso di utilizzo di tabelle, queste devono essere tutte numerate con numeri arabi e citate nel testo in ordine consecutivo (e.g. NON nominare le tabelle come Tabella $1 \mathrm{~A}, 1 \mathrm{~B}, \ldots$ o $1.0,1.1, \ldots$ ). Le tabelle devono essere presentate in formato editabile. Ciascuna tabella deve essere corredata da una breve didascalia; in caso di abbreviazioni, riportare una nota a piè di CIASCUNA tabella che spieghi TUTTE le abbreviazioni presenti in ognuna.

- In caso di utilizzo di figure, queste devono essere inviate in formato tiff o .jpg, allegate al manoscritto in singoli files, secondo le seguenti specifiche:

i) a colori (salvate in modalità CMYK): minimo 300 dpi di risoluzione;

ii) in bianco e nero: minimo $600 \mathrm{dpi}$ di risoluzione;

iii) minimo $17,5 \mathrm{~cm}$ di larghezza.

Ciascuna figura deve essere corredata da una breve didascalia.

$N B$ : In caso di Tabelle/Figure riprese e/o modificate da altri lavori già pubblicati, sarà cura degli autori accertarsi se tali materiali siano o meno coperti da copyright e procurarsi i permessi necessari per la riproduzione. Tali permessi dovranno essere allegati alla versione definitiva del lavoro. L'ufficio editoriale si riserva la facoltà di rimuovere Tabelle/Figure coperte da copyright, se sprovviste dei necessari permessi.

- In caso di utilizzo di abbreviazioni, la prima volta che esse sono citate è necessario scrivere per esteso la definizione+abbreviazione tra parentesi tonde [e.g. risonanza magnetica $(\mathrm{RMN})]$, a seguire si dovrà riportare solo l'abbreviazione (unica eccezione: nei titoli e nelle didascalie di tabelle e figure NON si utilizzano abbreviazioni).

\section{BIBLIOGRAFIA}

Le voci bibliografiche devono essere formattate secondo lo stile Vancouver.

Nella sezione Bibliografia, le voci bibliografiche devono essere numerate consecutivamente nell'ordine in cui appaiono per la prima volta nel testo (NON in ordine alfabetico) e, nel testo, devono essere indicate con numeri arabi in apice. Voci bibliografiche riferite a comunicazioni personali o dati non pubblicati devono essere incorporate nel testo e NON inserite tra le voci numerate [e.g. (Wright 2011, dati non pubblicati) o (Wright 2011, comunicazione personale)]. Le voci bibliografiche nella sezione Bibliografia devono tassativamente essere preparate come segue:

i) più di 3 autori, citare 3 autori, et al. Se il lavoro contiene solo 4 autori, citarli tutti e 4 ;

ii) titolo del lavoro in stampatello minuscolo;

iii) nome della rivista, senza punti, abbreviato secondo gli standard internazionali; in caso di dubbi sulla corretta abbreviazione, fare riferimento ai seguenti siti:

a. ISI Journal Abbreviations Index (http://library.caltech.edu/ reference/abbreviations/);

b. Biological Journals and Abbreviations (http://home.ncifcrf. gov/research/bja/);

c. Medline List of Journal Titles (ftp://ftp.ncbi.nih.gov/pubmed/J_Medline.txt);

iv) inserire l'anno di pubblicazione subito dopo il nome della rivista, seguito da punto e virgola;

v) NON inserire giorno o mese di pubblicazione;

vi) citare solo il volume, seguito dai due punti (NON citare il fascicolo tra parentesi);

vii) abbreviare le pagine, e.g. 351-8

Per accertarsi di aver correttamente formattato le voci bibliografiche, confrontarle con le citazioni in PubMed (http://www.ncbi. nlm.nih.gov/pubmed).

Esempi (prestare attenzione anche alla punteggiatura):

Articolo standard su Rivista

Halpern SD, Ubel PA, Caplan AL. Solid-organ transplantation in HIV-infected patients. N Engl J Med 2002;347:284-7.

Proceedings

Christensen S, Oppacher F. An analysis of Koza's computational effort statistic for genetic programming. In: Foster JA, Lutton E, Miller J, Ryan C, Tettamanzi AG, eds. Genetic programming. EuroGP 2002: Proceedings of the 5th European Conference on Genetic Programming, 2002 Apr 3-5, Kinsdale, Ireland. Berlin: Springer; 2002. pp 182-91.

Articoli i cui autori sono Organizzazioni

Diabetes Prevention Program Research Group. Hypertension, insulin, and proinsulin in participants with impaired glucose tolerance. Hypertension 2002;40:679-86.

Libri

Murray PR, Rosenthal KS, Kobayashi GS, Pfaller MA. Medical microbiology. 4th ed. St. Louis, MO: Mosby; 2002. (CITAZIONE DEL LIBRO INTERO)

Meltzer PS, Kallioniemi A, Trent JM. Chromosome alterations in human solid tumors. In: Vogelstein B, Kinzler KW, eds. The genetic basis of human cancer. New York, NY: McGraw-Hill; 2002. pp 93113. (CITAZIONE DI UN CAPITOLO)

\section{MODALITÀ D'INVIO DEI LAVORI}

Monografie

Gli Autori dovranno fare riferimento all'Editor-in-Chief o alle persone da lui designate nelle lettere di invito a scrivere gli articoli programmati.

Per gli articoli inviati da giovani internisti, gli Autori dovranno fare riferimento al Dr. Michele Meschi (e-mail: mmeschi@ausl.pr.it)e agli eventuali altri referenti da lei designati, nelle prime fasi di stesura dei manoscritti, revisioni e correzioni. 


\section{QUADERNI}

dell'Italian Journal

of Medicine

Il Dr. Meschi raccoglierà poi le versioni definitive dei lavori di ciascuna monografia e provvederà all'invio di tutti i materiali all'ufficio editoriale.

I lavori solo nella loro versione definitiva e approvata dalla Commissione FADOI Giovani dovranno pervenire all'ufficio editoriale già pronti per l'impaginazione e immediata pubblicazione (già corredati da eventuali permessi per la riproduzione di tabelle e immagini redatti secondo le presenti linee guida).

Traduzioni

Previo invito dell'Editor-in-Chief, gli Autori dovranno far pervenire all'ufficio editoriale la versione tradotta in italiano, al seguente indirizzo e-mail: paola.granata@pagepress.org

Il file in formato Word dovrà essere formattato secondo gli standard editoriali della rivista ufficiale ed essere già pronto per impagina- zione e immediata pubblicazione (corredato da eventuali permessi per la riproduzione di tabelle e immagini).

Si prega di inviare le eventuali tabelle in formato editabile e le figure in alta definizione secondo gli standard sopra riportati.

\section{NOTA PER GLI AUTORI}

I lavori pubblicati sui Quaderni ITJM non verranno indicizzati, ma saranno liberamente disponibili in un'apposita sezione del sito FADOI (http://www.fadoi.org/) e della rivista ufficiale.

Gli Autori i cui lavori siano accettati per la pubblicazione sui Quaderni ITJM e che fossero interessati a vederli pubblicati anche sulla rivista ufficiale, dovranno sottomettere attraverso il sito dell'ITJM (www.italjmed.org) la versione (già tradotta) in inglese e redatta in modo conforme alle linee guida della rivista; seguiranno poi la procedura di selezione tramite peer review e, se accettati, saranno inseriti nel piano editoriale standard.

\section{STAFF EDITORIALE}

Paola Granata, Journal Manager

paola.granata@pagepress.org

Claudia Castellano, Production Editor

Tiziano Taccini, Technical Support
PUBBLICATO DA

PAGEPress Publications

via A. Cavagna Sangiuliani 5

27100 Pavia, Italy

T. +39.0382 .464340$

F: +39.0382 .34872$

\section{agepress}

www.pagepress.org info@pagepress.org

\section{QUADERNI - ITALIAN JOURNAL OF MEDICINE}

Tutti gli articoli pubblicati sui QUADERNI - Italian Journal of Medicine sono redatti sotto la responsabilità degli Autori. La pubblicazione o la ristampa degli articoli della rivista deve essere autorizzata per iscritto dall'editore. Ai sensi dell'art. 13 del D.Lgs 196/03, i dati di tutti i lettori saranno trattati sia manualmente, sia con strumenti informatici e saranno utilizzati per l'invio di questa e di altre pubblicazioni e di materiale informativo e promozionale. Le modalità di trattamento saranno conformi a quanto previsto dall'art. 11 del D.Lgs 196/03. I dati potranno essere comunicati a soggetti con i quali PAGEPress intrattiene rapporti contrattuali necessari per l'invio delle copie della rivista. Il titolare del trattamento dei dati è PAGEPress Srl, via A. Cavagna Sangiuliani 5 - 27100 Pavia, al quale il lettore si potrà rivolgere per chiedere l'aggiornamento, l'integrazione, la cancellazione e ogni altra operazione di cui all'art. 7 del D.Lgs 196/03.

https://www.italjmed.org/index.php/ijm/quad 
PAGEPress - Scientific Publications

Via A. Cavagna Sangiuliani, 5 - 27100 Pavia - Italy

Phone +390382 464340 - Fax +390382 34872

www.pagepress.org 